\title{
Topology Abstraction Service for IP Virtual Private Networks
}

By

Ravishankar Ravindran, B. of Tech, M.Sc.

\author{
A thesis submitted to \\ The Faculty of Graduate Studies and Research \\ in partial fulfilment of \\ the degree requirements of \\ Doctor of Philosophy in Electrical Engineering \\ Ottawa-Carleton Institute for \\ Electrical and System Engineering \\ Department of Systems and Computer Engineering \\ Carleton University \\ Ottawa, Ontario, Canada \\ January 2009
}

Copyright $(\mathcal{C}$

2009 - Ravishankar Ravindran 
Library and Archives Canada

Published Heritage Branch

395 Wellington Street Ottawa ON K1A 0N4 Canada
Bibliothèque et

Archives Canada

Direction du

Patrimoine de l'édition

395, rue Wellington

Ottawa ON K1A ON4

Canada
Your file Votre référence
ISBN: $978-0-494-60118-1$
Our file Notre référence
ISBN: $978-0-494-60118-1$
NOTICE:

The author has granted a nonexclusive license allowing Library and Archives Canada to reproduce, publish, archive, preserve, conserve, communicate to the public by telecommunication or on the Internet, loan, distribute and sell theses worldwide, for commercial or noncommercial purposes, in microform, paper, electronic and/or any other formats.

The author retains copyright ownership and moral rights in this thesis. Neither the thesis nor substantial extracts from it may be printed or otherwise reproduced without the author's permission.
AVIS:

L'auteur a accordé une licence non exclusive permettant à la Bibliothèque et Archives Canada de reproduire, publier, archiver, sauvegarder, conserver, transmettre au public par télécommunication ou par l'Internet, prêter, distribuer et vendre des thèses partout dans le monde, à des fins commerciales ou autres, sur support microforme, papier, électronique et/ou autres formats.

L'auteur conserve la propriété du droit d'auteur et des droits moraux qui protège cette thèse. $\mathrm{Ni}$ la thèse ni des extraits substantiels de celle-ci ne doivent être imprimés ou autrement reproduits sans son autorisation.
In compliance with the Canadian Privacy Act some supporting forms may have been removed from this thesis.

While these forms may be included in the document page count, their removal does not represent any loss of content from the thesis.
Conformément à la loi canadienne sur la protection de la vie privée, quelques formulaires secondaires ont été enlevés de cette thèse.

Bien que ces formulaires aient inclus dans la pagination, il n'y aura aucun contenu manquant. 


\section{Abstract}

VPN service providers (VSP) and IP-VPN customers have traditionally maintained service demarcation boundaries between their routing and signaling entities, and this has resulted in the VPNs viewing the VSP network as an opaque entity and therefore limiting any meaningful interaction between the VSP and the VPNs. The purpose of this research is to address this issue by enabling a VSP to share its core topology information with the VPNs as a service in a manner which is both practical and scalable. We use the notion of topology abstraction, which serves as a means of sharing the core topology information as abstract graphs associated with QoS metric information with the VPNs.

We validate this idea of enabling topology information sharing with the IP-VPNs as a topology abstraction service in the context of a challenging framework called managed dynamic VPN framework. The abstraction provided to a VPN as a TA service is generated using a novel process that enables fairness and service differentiation among the VPN customers.

New problems arise when topology abstraction is provided as a managed service to the IP-VPNs, as the available core resources need to be summarized for advertising to VPN customers while maintaining maximum session performance and core resource utilization. These problems are addressed through novel topology abstraction schemes. 
To my wife and parents 


\section{Acknowledgements}

I would like to sincerely thank my thesis advisors Prof. Changcheng Huang and Prof. Krishnaiyan Thulasiraman for their continuous guidance and support during the course of this thesis. 


\section{Table of Contents}

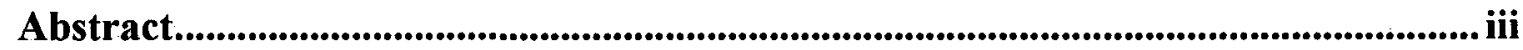

Acknowledgements .......................................................................................................................... v

Table of Contents .................................................................................................................. vi

List of Tables ........................................................................................................................... ix

List of Figures................................................................................................................................. $\times$

List of Notations .......................................................................................................... xvii

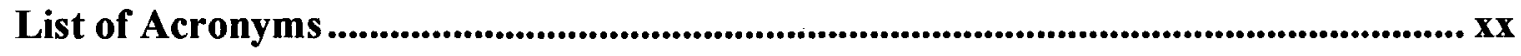

Chapter 1 Introduction..................................................................................................................... 1

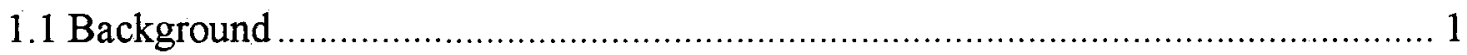

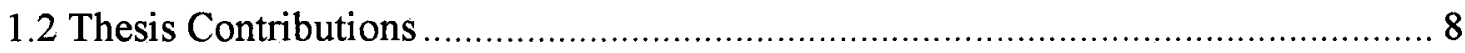

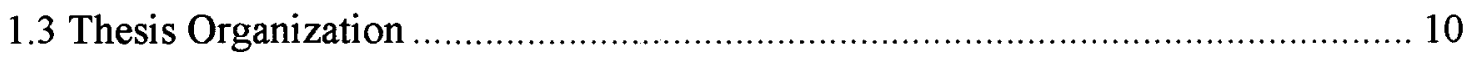

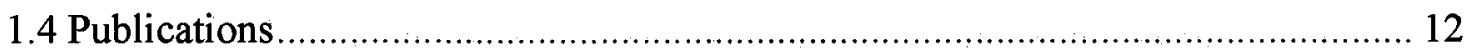

Chapter 2 IP Virtual Private Networks and Topology Aggregation for Hierarchical

Routing.................................................................................................................................................................... 13

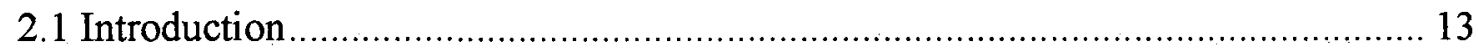

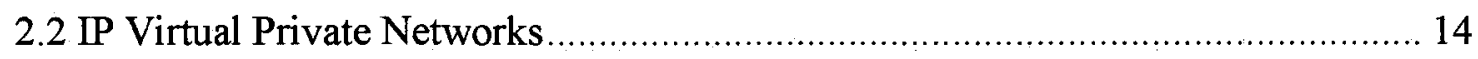

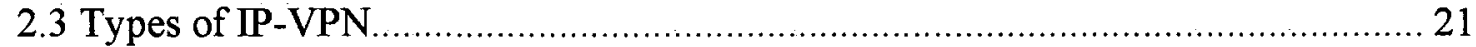

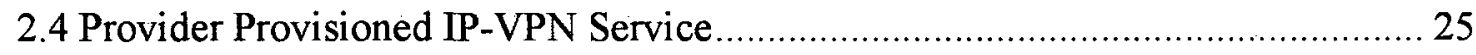




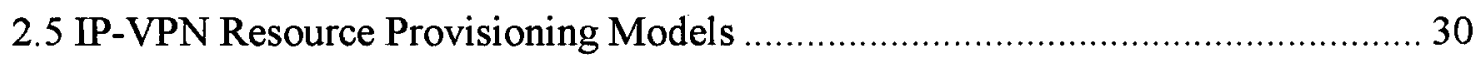

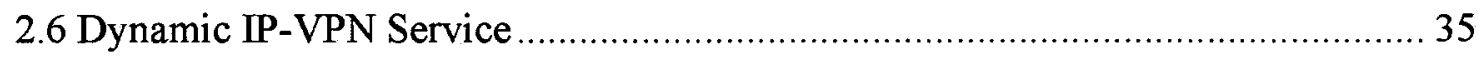

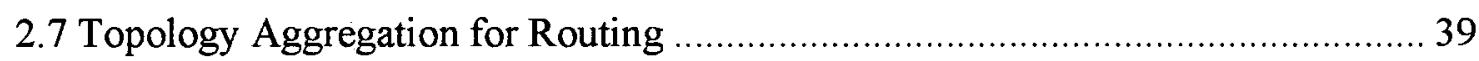

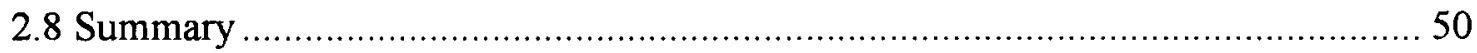

Chapter 3 Topology Abstraction Service for IP-VPNs: Definition and Realization 52

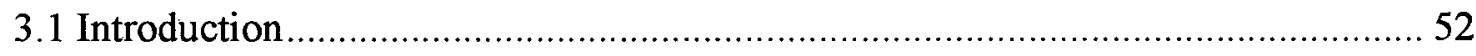

3.2 Reachability Abstraction in Managed IP-VPN Service ...................................... 53

3.3 Topology Abstraction Service for IP-VPNs .................................................. 55

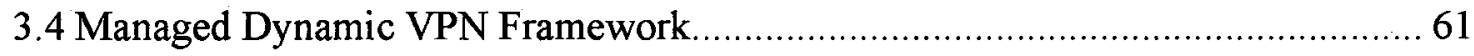

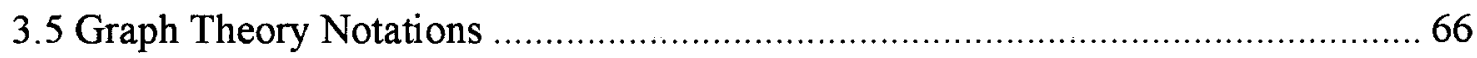

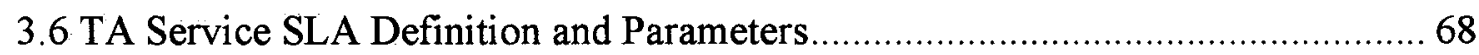

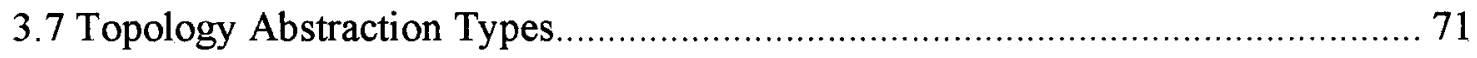

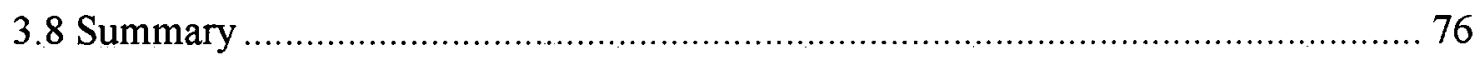

Chapter 4 Topology Abstraction Generation and Evaluation......................................... 78

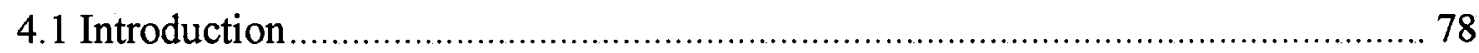

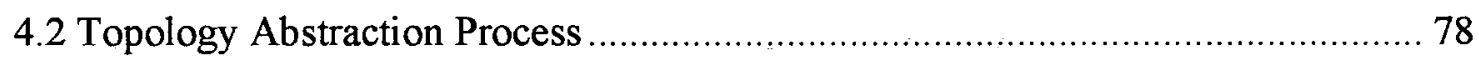

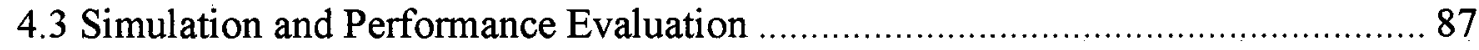

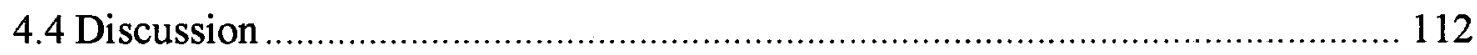

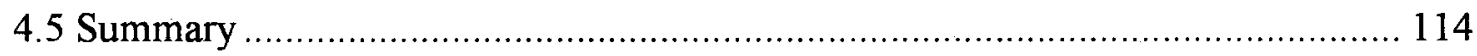

Chapter 5 Decentralized VPN Topology Abstraction ...................................................... 117

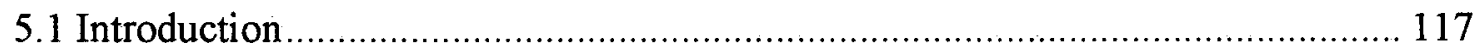

5.2 VPN Topology Abstraction Problem ............................................................ 118

5.3 Simulation and Performance Evaluation ....................................................... 140

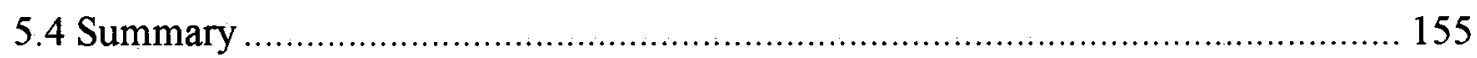

Chapter 6 Centralized VPN Topology Abstraction........................................................ 157

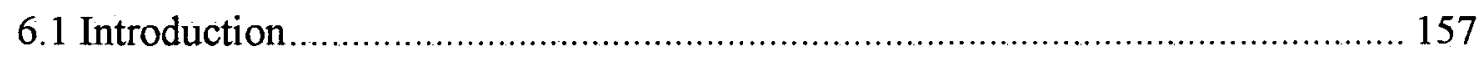

6.2 Centralized Topology Abstraction Process.................................................... 158 
6.3 VPN Core Capacity Sharing Problem........................................................... 160

6.4 Improving Fairness of MMCF Based Partitioning Heuristic.............................. 175

6.5 Simulation and Performance Evaluation ......................................................... 186

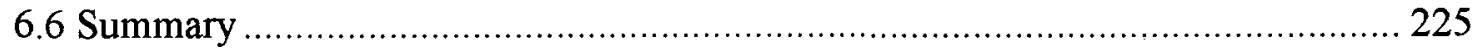

Chapter 7 Conclusions and Future Work ............................................................................ 228

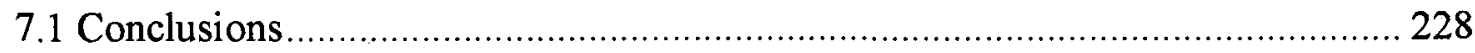

7.2 Future Work

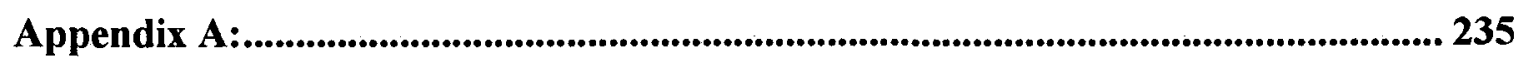

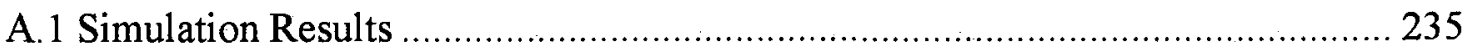

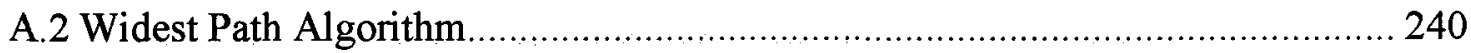

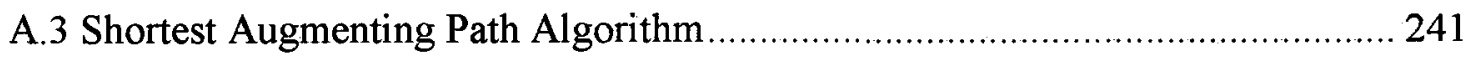

A. 4 Maximum $M$-Route Flow Algorithm ...................................................... 241

A. 5 Nearest Node First Steiner Tree Algorithm …….......................................... 243

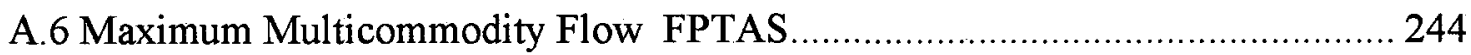

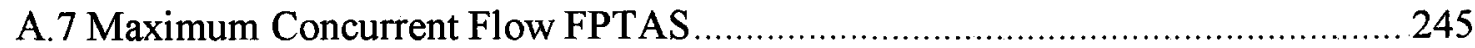

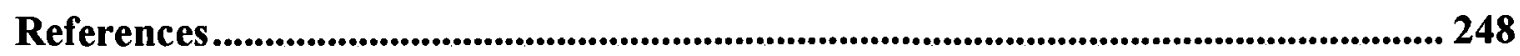




\section{List of Tables}

Table 4.1: Simulation parameters to evaluate TA service and effectiveness of TA-SLA

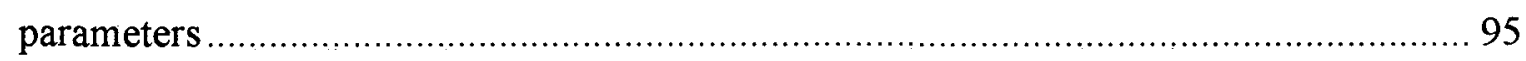

Table 5.1: Simulation parameters to evaluate decentralized abstraction schemes ......... 142

Table 6.1: Simulation parameters to evaluate centralized abstraction schemes ............ 190 


\section{List of Figures}

Figure 2-1: High level view of managed site-to-site IP-VPN service ............................. 16

Figure 2-2: High level view of CPE based IP-VPN service .................................... 22

Figure 2-3: High level view of provider provisioned IP-VPN service .......................... 25

Figure 2-4: Example for PNNI topology aggregation ................................................... 44

Figure 2-5: PNNI multi-level hierarchical topology aggregation.................................. 44

Figure 2-6: Example of hierarchical routing in OSPF .............................................. 49

Figure 3-1 (a-c): Reachability abstraction in network based IP-VPN .......................... 55

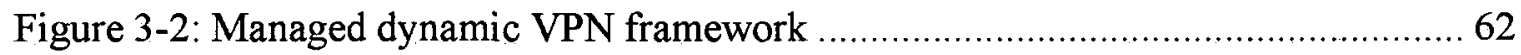

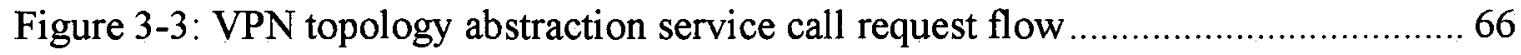

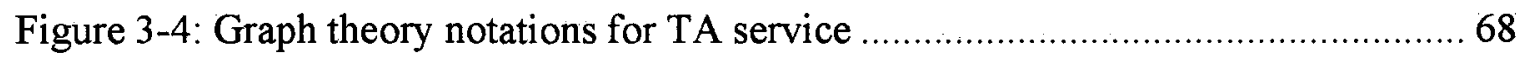

Figure 3-5: VSP providing topology abstraction service ............................................... 72

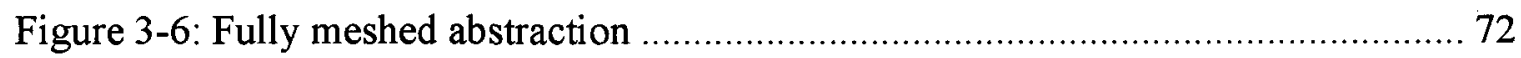

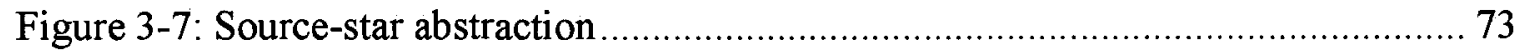

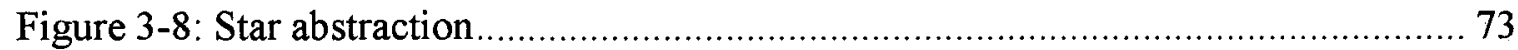

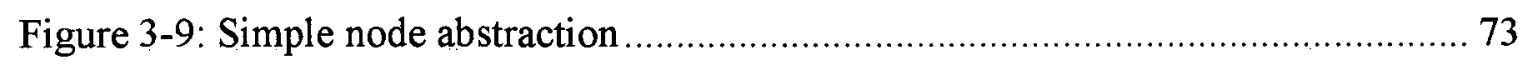

Figure 4-1: Topology abstraction process for TA Service............................................. 86

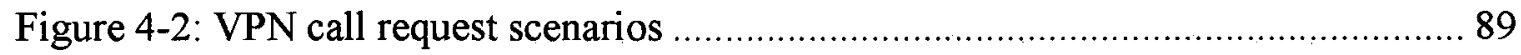

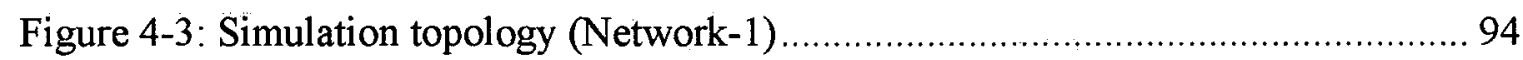

Figure 4-4: Simulation topology (Network-2) ……................................................ 94

Figure 4-5: Simulation topology (Network-3 )......................................................... 95

Figure 4-6: Objective 1, VPN call performance comparing success ratio with varying

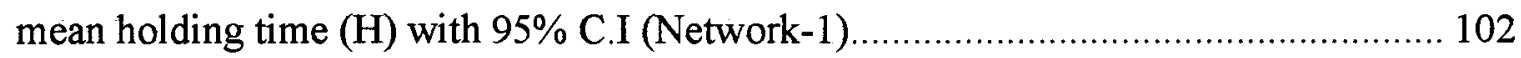

Figure 4-7: Objective 1, VPN call performance comparing crankback ratio with varying mean holding time $(\mathrm{H})$ with 95\% C.I (Network-1) .................................................. 102 
Figure 4-8: Objective 1, VPN call performance comparing misscall ratio with varying mean holding time $(\mathrm{H})$ with $95 \%$ C.I (Network-1).

Figure 4-9: Objective 1, VSP core network utilization with varying mean holding time (H) with 95\% C.I (Network-1)

Figure 4-10 (a-b): Objective 1, Comparison of success ratio and (crankback + misscall ratio) with varying mean holding time $(\mathrm{H})$ with $95 \%$ C.I (Network-2).....

Figure 4-11 (a-b): Objective 1, Comparison of success ratio and (crankback + misscall ratio) with varying mean holding time $(\mathrm{H})$ with $95 \%$ C.I (Network-3). 104

Figure 4-12: Objective 1, Fairness performance in terms of standard deviation of call performance metrics with varying mean holding time $(\mathrm{H})$ with 95\% C.I (Network-1). 106 Figure 4-13: Objective 1, Comparing VSP network utilization for different abstraction types with varying mean holding time $(\mathrm{H})$ with $95 \%$ C.I (Network-1).

Figure 4-14: Objective 2, VPN call performance comparing success ratio with varying refresh update interval with $95 \%$ C.I (Network-1) 109 Figure 4-15: Objective 2, VPN call performance (crankback+ misscall) ratio with varying refresh update interval with $95 \%$ C.I (Network-1). 109 Figure 4-16: Objective 3, VPN success ratio with varying refresh update interval and varying abstraction type with varying mean holding time $(\mathrm{H})$ with $95 \%$ C.I (Network-1)

Figure 4-17: Objective 3, VPN (crankback + misscall) ratio with varying refresh update interval and varying abstraction type with varying mean holding time $(\mathrm{H})$ with $95 \%$ C.I (Network-1) 111

Figure 5-1: VSP providing topology abstraction service to red and blue VPN. 119

Figure 5-2: Fully meshed abstraction for red VPN.

Figure 5-3: Fully meshed abstraction for blue VPN.

Figure 5-4: Maximum capacity abstraction scheme for VPN topology abstraction problem with reference to Figure 4.1. 126

Figure 5-5: M-Route flow example 
Figure 5-6: Mixed bound scheme for VPN topology abstraction problem with reference to Figure 4.1

Figure 5-7: Steiner tree based scheme for VPN topology abstraction problem with reference to Figure 4.1

Figure 5-8: VPN call performance comparing VPN success ratio with varying mean

holding time $(\mathrm{H})$ with $95 \%$ C.I (Network-1) . 145

Figure 5-9: VPN call performance comparing VPN success ratio with varying bandwidth request (X) with $95 \%$ C.I (Network-1).

Figure 5-10: VPN call performance comparing VPN crankback ratio with varying mean holding time $(\mathrm{H})$ with $95 \%$ C.I (Network-1)

Figure 5-11: VPN call performance comparing VPN crankback ratio with varying bandwidth request (X) with $95 \%$ C.I (Network-1)

Figure 5-12: VPN call performance comparing VPN misscall ratio with varying mean holding time $(\mathrm{H})$ with $95 \%$ C.I (Network-1) 149

Figure 5-13: VPN call performance comparing VPN misscall ratio with varying bandwidth request (X) with $95 \%$ C.I (Network-1)

Figure 5-14: Comparing VSP core network utilization with varying mean holding time (H) with 95\% C.I (Network-1).

Figure 5-15: Comparing VSP core network utilization with varying bandwidth request $(X)$ with $95 \%$ C.I (Network-1).

Figure 5-16(a-b): Success and crankback ratio with varying mean holding time $(\mathrm{H})$ with 95\% C.I (Network-2) 153

Figure 5-17(a-b): Miscall ratio and network utilization with varying mean holding with 95\% C.I time (H) (Network-2). 153

Figure 5-18(a-b): Success and crankback ratio with varying mean holding time $(\mathrm{H})$ with 95\% C.I (Network-3) 153

Figure 5-19(a-b): Miscall ratio and network utilization with varying mean holding time

(H) with 95\% C.I (Network-3). 154

Figure 6-1: VPN subgraph partition computation steps executed by the central server. 160 
Figure 6-2: MConF based partitioning heuristic for VPN core capacity sharing problem

Figure 6-3: Example demonstrating MConF drawback

Figure 6-4: MMCF based partitioning heuristic for VPN core capacity sharing problem

Figure 6-5: Logical view of the flow balancing process. 180

Figure 6-6: Flow balancing heuristic for fair partitioning problem

Figure 6-7: Objective 1, Performance of MConF FPTAS with varying approximation factor (Network-1) 192

Figure 6-8: Objective 1, Comparing MConF FPTAS and LP optimal solution with varying approximation factor (Network-1). 192

Figure 6-9: Objective 2(a), VSP abstraction efficiency and core network utilization with varying mean holding time $(\mathrm{H})$ for $\mathrm{MConF}$ based abstraction scheme with $95 \%$ C.I (Network-1) 194

Figure 6-10: Objective 2(a), VPN call performance with varying mean holding time $(\mathrm{H})$ for MConF based abstraction scheme with 95\% C.I (Network-1) 195 Figure 6-11: Objective 2(a), VSP abstraction efficiency and core network utilization with varying bandwidth request $(\mathrm{X})$ for $\mathrm{MConF}$ based abstraction scheme with $95 \% \mathrm{C}$.I (Network-1) 195

Figure 6-12: Objective 2(a), VPN call performance with varying bandwidth request (X) for MConF based abstraction scheme with 95\% C.I (Network-1) 196 Figure 6-13: Objective 2(b), Comparison between the achieved throughputs of MConF and MMCF FPTAS with varying size of graph 198 Figure 6-14: Objective 2(b), Throughput comparison of MConF and MMCF based abstraction schemes from a simulation run (Network-1). 198 Figure 6-15: Objective 2(b), VPN call performance comparison of success ratio MConF and MMCF based abstraction schemes with varying mean holding time $(\mathrm{H})$ with $95 \%$ C.I (Network-1) 199 
Figure 6-16: Objective 2(b), VPN call performance (crankback + misscall) ratio comparison of MConF and MMCF based abstraction schemes with varying mean holding time $(\mathrm{H})$ with $95 \%$ C.I (Network-1) 199

Figure 6-17: Objective 2(b), VSP network utilization comparison of MConF and MMCF based abstraction schemes with varying mean holding time $(\mathrm{H})$ with $95 \%$ C.I (Network1) 200

Figure 6-18: Objective 2(b), Comparison of fairness of MConF and MMCF based abstraction schemes with varying mean holding time (H) with 95\% C.I (Network-1) .. 200 Figure 6-19: Objective 2(b), Comparison of abstraction efficiency of MConF and MMCF based abstraction schemes with varying mean holding time $(\mathrm{H})$ with $95 \%$ C.I (Network1) 201

Figure 6-20 (a-b): Objective 2(b), Comparison of success, crankback and misscall ratio of MConF and MMCF based abstraction schemes with varying mean holding time $(\mathrm{H})$ with 95\% C.I (Network-2) 202

Figure 6-21 (a-b): Objective 2(b), Comparison of abstraction efficiency and network utilization of MConF and MMCF based abstraction schemes with varying mean holding time $(\mathrm{H})$ with $95 \%$ C.I (Network-2) 202

Figure 6-22 (a-b): Objective 2(b), Comparison of success, crankback and misscall ratio of $\mathrm{MConF}$ and $\mathrm{MMCF}$ based abstraction schemes with varying mean holding time $(\mathrm{H})$ with 95\% C.I (Network-3) 203

Figure 6-23: Objective 2(b), Comparison of abstraction efficiency and network utilization of MConF and MMCF based abstraction schemes with varying mean holding time $(\mathrm{H})$ with $95 \%$ C.I (Network-2) 203

Figure 6-24: Objective 3(a), Offline comparison of abstraction efficiency of MConF, MMCF, MMCF with MB1/MB2 formulations for different graphs 205

Figure 6-25: Objective 3(a), Offline comparison of fairness of MConF, MMCF, MMCF with MB1/MB2 formulations for different graphs 206 Figure 6-26: Objective 3(b), Comparing variance of commodity throughputs of MMCF with and without Fair Balancing for different graphs 208 
Figure 6-27: Objective 3(b), Comparing VSP abstraction efficiency of MMCF with and without Fair Balancing with varying mean holding time $(\mathrm{H})$ with 95\% C.I (Network-1)

Figure 6-28: Objective 3(b), VPN call performance comparing success ratio of MMCF with and without Fair Balancing with varying mean holding time $(\mathrm{H})$ with $95 \%$ C.I (Network-1). 209

Figure 6-29: Objective 3(b), Comparing fairness of MMCF with and without Fair Balancing with varying mean holding time $(\mathrm{H})$ with $95 \%$ C.I (Network-1). 209 Figure 6-30: Objective 4(a), VPN call performance comparing success ratio of maximum capacity, MConF and MMCF based TA schemes with varying mean holding time $(\mathrm{H})$ with $95 \%$ C.I (Network-1) 213

Figure 6-31: Objective 4(a), VPN call performance comparing crankback ratio of maximum capacity, MConF and MMCF based abstraction schemes with varying mean holding time $(\mathrm{H})$ with $95 \%$ C.I (Network-1)

Figure 6-32: Objective 4(a), VPN call performance comparing misscall ratio of maximum capacity, MConF and MMCF based TA schemes with varying mean holding time $(\mathrm{H})$ with $95 \%$ C.I (Network-1)

Figure 6-33: Objective 4(a), VSP network utilization comparison of maximum capacity, MConF and MMCF based TA schemes with varying mean holding time $(\mathrm{H})$ with $95 \%$ C.I (Network-1)

Figure 6-34: Objective 4(b), VPN call performance comparing success ratio of MConF based abstraction scheme with varying oversubscription factor $(\mathrm{Y})$ with $95 \%$ C.I (Network-1). 220

Figure 6-35: Objective 4(b), VPN call performance comparing success ratio of MMCF based abstraction scheme with varying oversubscription factor $(\mathrm{Y})$ with $95 \% \mathrm{C}$.I (Network-1). 220

Figure 6-36: Objective 4(b), VPN call performance comparing misscall ratio of MConF based abstraction scheme with varying oversubscription factor $(\mathrm{Y})$ with $95 \%$ C.I (Network-1) 
Figure 6-37: Objective 4(b), VPN call performance comparing crankback ratio of MConF based abstraction scheme with varying oversubscription factor $(\mathrm{Y})$ with $95 \%$ C.I (Network-1)

Figure 6-38: Objective 4(b), VSP network utilization comparison of MConF based abstraction scheme with varying oversubscription factor (Y) with 95\% C.I (Network-1)

Figure 6-39: Objective 4(b), Success ratio comparison of MConF/MMCF/MCS TA schemes with varying mean holding time $(\mathrm{H})$ with $95 \%$ C.I (Network-1) 222 Figure 6-40: Objective 4(b), Success ratio comparison of MConF/MMCF/MCS TA schemes with varying mean holding time (H) with 95\% C.I (Network-1) 223 


\section{List of Notations}

$G(V, E)$ : VSP core network

$B:$ Set of all border nodes

$t_{i, j}($ or $t(e))$ : Total capacity of edge $(i, j)$ or $e$

$r_{i, j}($ or $r(e))$ : Residual capacity of edge $(i, j)$ or $e$

$U$ : Set of all VPNs subscribing to topology abstraction service

$U_{b}:$ Set of VPNs hosted on node $b$

$C_{k}, P_{k}:$ Set of $\mathrm{CE} / \mathrm{PE}$ nodes corresponding to VPN $k$

$C_{k, b}$ : Set of CE nodes of VPN $k$ hosted on border node $b$

$T S_{k}$ : TA-SLA parameter set for VPN $k$

$T_{k}$ : Abstract topology type subscription of VPN $k$

$R_{k}:$ Abstract topology refresh interval for VPN $k$

$G_{k, l}\left(V_{k}, E_{k}\right)$ : Abstract topology of VPN $k$ of topology type $l$

$w_{k, l}(x, y):$ Bandwidth of the virtual link $(x, y) \in E_{k}$ of abstract topology type $l$ for VPN $k$

$Z(x, y)$ : Set of VPNs common to border nodes $x$ and $y$

$Z_{l}(x, y)$ : Set of VPNs common to border nodes $x$ and $y$ with same abstract topology type $l$

$\omega(x, y)$ : Capacity of maximum capacity path between nodes $x$ and $y$

$\alpha(x, y)$ : Maximum flow between nodes $x$ and $y$

$\alpha(k)$ : Maximum flow of source-destination commodity pair $\left(s_{k}, d_{k}\right)$

xvii 
$\delta_{k, l}(x, y)$ : Upper bound virtual link capacity estimate between nodes $x$ and $y$ in abstract graph $G_{k, l}\left(V_{k}, E_{k}\right)$ computed using mixed bound algorithm

$\gamma_{k, l}(x, y)$ : Lower bound virtual link capacity estimate between nodes $x$ and $y$ in abstract graph $G_{k, l}\left(V_{k}, E_{k}\right)$ computed using mixed bound algorithm

$\eta(x, y): M$-Route Flow between nodes $x$ and $y$

$K$ : Set of all commodities corresponding to a multicommodity flow formulation

$K_{v}$ : Set of all source-destination border node pair commodity of VPN $v$

$x_{i, j}^{v, k}:$ Resource partitioned on edge $(i, j)$ for VPN $v$ for source-destination commodity $k$

$x(e)$ : Aggregate commodity flow on edge $e$

$f_{v, k}$ : Total flow corresponding to source-destination pair commodity $k$ for VPN $v$

$D\left(s_{k}, d_{k}\right)(D(k))$ : Demand for commodity $k$ corresponding to source-destination commodity pair $\left(s_{k}, d_{k}\right)$

$\varepsilon:$ Desired approximation factor with respect to optimal solution for FPTAS algorithm

$\beta$ : Throughput of maximum multicommodity concurrent flow (MConF) formulation

$\beta_{k}$ : Throughput of commodity $k$ in modified MConF formulation

$f_{m}$ : Aggregate flow of all commodities of $\mathrm{MConF}$ formulation

$f_{m m}$ : Aggregate flow of all commodities of modified MConF formulation

$f_{m c}$ : Aggregate flow of all commodities of maximum multicommodity flow (MMCF) formulation

$f_{b}$ : Aggregate flow of all commodities achieved by balancing MMCF flows

$f_{x}(k)$ : Flow corresponding to commodity $k$ using multicommodity flow formulation $x$

$\sigma:$ Threshold value for flow balancing algorithm

$\Omega_{e} / \Omega_{d}:$ Excess/Deficit commodity set

xviii 
$\varphi_{k, l} / \varphi_{k, u}:$ Lower/Upper bound for commodity $k$ for bounded MMCF formulation $H$ : Mean holding time setting for simulation $X$ : Upper bound of uniformly distributed VPN bandwidth request for simulation $Y$ : Oversubscription factor of residual link capacity 


\section{List of Acronyms}

ATG: Abstract Topology Generation Module

CS: Central Server

CE: Customer premises Equipment

CAC: Call Admission Control

CAPEX: Capital Expenditure

CR-LDP: Constrained-based Label Distributed Protocol

DiffServ : Differentiated Service QoS framework

DVS: Dynamic VPN Service

IP-VPN: VPN Service to transport IP data

IntServ: Integrated Service QoS framework

MConF: Maximum Concurrent Flow

M-MConF: Modified Maximum Concurrent Flow

MMCF: Maximum Multicommodity Flow

MPLS: Multi-Protocol Label Switching

MDVF: Managed Dynamic VPN Framework

OSPF: Open Shortest Path First

OPEX: Operational Expenditure

PE: Provider Edge equipment

PP-VPN: Provider Provisioned VPN

PNNI : Private Network-to-Network Interface

QoS: Quality of Service

RSVP-TE: RSVP with TE extensions

SLS: Service Level Specification

SLA: Service Level Agreement

TA: Topology Abstraction

TA-SLA: Topology Abstraction SLA

TE: Traffic Engineering

VTD: VPN Topology Database

VPN-TA: VPN Topology Abstraction Problem

VPN-CS: VPN Core Capacity Sharing Problem

VRF: Virtual Routing and Forwarding

VPN: Virtual Private Network

VSP: VPN Service Provider

VSN: VSP's Network 


\section{Chapter 1 \\ Introduction}

\subsection{Background}

A virtual private network (VPN) [Yua01] refers to a logical network that connects entities of the same user group (enterprise, provider, etc...) that are geographically dispersed and overlaid over a shared transport network that is owned by a VPN service provider (VSP). A VPN is normally guarded by one or more service level agreement (SLA) attributes, which are related to control and data plane functions that guarantee a certain level of performance with respect to data security, quality of service (QoS), service availability, and service pricing requirements.

The traditional model of provisioning an IP-VPN relies heavily on site-to-site traffic estimates, which are made available to the VSP by a VPN customer. This site-to-site traffic estimate is then used to determine tunnel paths of constant bit rates between the border node pairs. As long as the traffic profile is less than the estimated traffic requirement, this site-to-site traffic estimate will deliver bandwidth guaranteed performance to the IP-VPN traffic. This mode of provisioning is called the PIPE model, 
and it has a few practical shortcomings. For example, as the number of VPN endpoints increase, managing the VPN traffic becomes troublesome. Also, since each of the pipes behaves like a dedicated virtual circuit, no multiplexing gain can be achieved over the tunnels for the traffic from the same VPN or from different VPNs. This scheme also requires a VPN to predict its traffic requirements well ahead of time in the form of a traffic matrix. Estimating the traffic matrix for each pair of sites is often a difficult task, which typically ends with the VPN customer quantifying the worst case traffic estimate; consequently, this often leads to unnecessary capital expenditure (CAPEX) on behalf of the VPN and poor network utilization of the VSP's network resources.

In order to address the drawbacks of the PIPE model, the HOSE model was first proposed in [Duf95]. It was proposed with the intention of easing traffic specifications, improving statistical multiplexing, and allowing flexibility in terms of traffic distribution from a VPN end point to all other end points. In the case of a HOSE model, the VPN customer specifies the aggregate traffic requirements for each of its end points peering with the PE (Provider Edge) node of the VSP. The aggregate specification is in the form of an ordered pair for each VPN site. The first element is the maximum traffic ingressing (entering) the VSP's network from the VPN peering point and destined to all the other end points of the VPN. The second element represents the maximum traffic egressing (leaving) an end point into the VPN site. This corresponds to the traffic originating from all the other end points of the VPN. Though this form of traffic specification eases the problem of traffic estimation for the VPN customers, it is a challenge for the providers to use this form of traffic specification to provision the network so as to maximize statistical 
multiplexing and meet the aggregate ingress/egress bandwidth requirement of each VPN end point.

Another traffic specification model called the RANGE model [Kha00] allows the VPN to specify the requirements as a range of quantitative services. This model builds on the DiffServ model [RFC2475] of providing QoS. Even in this case, the VPN is not required to predict and specify any peak traffic requirements as in case of the PIPE model.

Previously discussed modes of VPN provisioning is generally good for IP-VPNs whose traffic specification changes at longer time scale. With increasing deployment of short lived and bandwidth intensive multimedia applications in enterprise VPNs, an approach designed to enable the VPN sites to negotiate SLA and seek services dynamically is required. In this thesis, a novel framework that enables dynamic VPN service (DVS) in a managed IP-VPN context is proposed.

Earlier research on enabling DVS was limited to studying the problem of enabling sharing of link capacity dynamically among VPNs. Initial proposals, such as in [Mit99] [Gar00], proposed solutions which allow dynamic resizing of the pre-established virtual circuits as a function of varying traffic demands of the VPNs, and thereby improving the statistical multiplexing gain and the overall utilization of the core network.

A more recent line of research in the existing literature, which enables DVS, is the work related to programmable VPNs; this work allows VPNs to have access to a subset of the router and network resources that can be optimized in order to satisfy the interests of the customer's applications. The goal of this area of work is to provide the flexibility 
that the VPNs needs in order to effectively control the provisioned switch and link capacity resources dedicated to the VPNs in the VSP's network. Such a framework allows the VPNs to utilize the committed resources sensibly; furthermore, this framework enables rapid SLA negotiation and provision newer capacity requests in smaller timescales. [Isa00] proposed a dynamic programmable VPN architecture that allows spawning dynamic VPN networks with dedicated router and link resources built over logical switch partitions called switchlets controlled by an open control interface. These programmable VPN architectures and solutions enabling dynamic VPN creation assume availability of access to physical router and link resources through an open programmable interface; for this reason, employing this approach is not possible when strict trust issues exist, as in the case of a VPN enterprise and a transport infrastructure owned by a commercial VSP. Another drawback with this mode of VPN management is similar to that of the PIPE model which requires prior knowledge of the demand matrix of the VPN, which is then mapped to the appropriate switch and link resources in order to spawn VPNs dynamically and enable customers to manage it.

In this thesis, we propose a novel service that enables VSP to share its core topology and link state information in a scalable manner with its IP-VPN customers. This service is enabled by the novel application of topology abstraction, which is on the lines of the topology aggregation concept applied for hierarchical routing, but new under the context of IP-VPN service. This topology abstraction service is studied in the context of a novel challenging framework called managed dynamic VPN framework which enables it to be 
realized in a VSP's network. The VPNs uses the abstracted topology and QoS metric information to seek services on demand from the VSP. This mode of provisioning prevents wastage of any committed resources, which may occur as a result of poor utilization in a dynamic call request scenario if well known models of provisioning based on the PIPE or the HOSE model is used; it also enables statistical multiplexing of VPN demands in the core of the VSP's network resulting in improved use of the available network resources.

Before providing a summary of our contributions, we provide a high level discussion on certain pros and cons of providing the TA service. These points are further discussed with the support of simulation analysis in Section 4.4 of Chapter 4 .

\subsubsection{Discussion on TA Service}

The benefits of the TA service are:

- The goal of VPN is to provide a customer a virtual private network that forms an overlay network over some existing public networks. The customer should have full control over this overlay network in terms of routing and content distribution in such a way that the overlay network looks like their own network. This was traditionally achieved through leased line services. However leased line services do not allow sharing and result in higher cost for the customer. The L3 VPN tries to solve this problem through provider-based VPN which gives the provider full control of all the VPNs sharing the same provider network. While this approach can maximize the sharing of the 
provider's resources, the customer does not have any information about the underlined network and therefore the overlay network is poorly utilized.

Our topology abstraction solution provides a compromised solution between the above two extreme approaches. It allows the VPN customers to choose the kind of information granularity they need for their VPNs so that they can utilize the overlay VPNs more efficiently in terms of routing information among different VPN sites and distributing application contents through out their VPNs. The customers who want to make their VPNs more like their own networks will pay more money to the provider for more TA information. This will allow them to route their traffic and distribute their application contents more efficiently in the overlay network and therefore lead to less bandwidth cost of the underlined provider's networks. In the end, they may save more in terms of overall cost. On the other hand, our approach still allows providers to multiplex different VPNs and achieve maximum sharing of their network resources while keeping their physical topology of the VSP confidential.

This thesis will focus on the issues related to the provisioning of TA in the provider's network. The design of overlay network is another major research area and it is beyond the scope of this thesis.

- Providing TA information to the VPNs allows them to seek resources from the VSP with high degree of success. This will result in achieving the goal of enabling VPNs to make routing decision with very high efficiency, leading to good VPN experience. This will be demonstrated by very good success ratio (for performance metric definitions, refer to Section 4.3.1) performance achieved by the VPNs. The overall gain in success 
ratio is achieved because of the gain achieved both in terms of crankback and misscall ratio performance for the VPNs. Also, the VPNs subscribing to TA information with varying granularity demonstrate different levels of service performance. This allows VSP to achieve service differentiation through TA service.

- In current provider networks control plane scalability is a major concern. With TA service, as we will observe, the VPNs achieve very good crankback ratio performance compared to VPNs that are not provided with any form of abstraction. This gain is significant as it reduces the call processing burden on the VSP which would otherwise be required to process these calls. This results in cost savings to the VSP, which increases with increasing number of VPNs served by the VSP.

Though the above points make very good argument to provide TA service, we draw attention to a few drawbacks with it. This we summarize next.

- Though the TA service achieves the objective of improved decision making by the VPNs provided with TA information, it may lead to poor judgement on the part of the VPNs at higher network load conditions. An increase in misscall and crankback ratios may occur with increase in network load. However, as we will show in Chapter 4, the deterioration of these performance metrics is small relative to the overall gain in terms of success ratio achieved compared to the case when no TA is applied. This is the case even at high load conditions. It will also be observed that the misscall and crankback ratio performance also vary with granularity of TA information based on which a VPN makes the routing decisions. Specifically, VPNs provided with lower granular abstraction 
performs poorly in terms of routing decisions when compared to those provided with higher granular abstractions.

- Another observation made in the context of the TA service is that it may lead to lesser network utilization compared to the case when the VSP provides no TA service. However, as we will observe later, the reduction in the average utilization is not found to be very significant. This reduction in network utilization is offset by very good success ratio achieved by the VPNs provided with some form of TA information.

- The VSP and the VPN bear a control overhead cost associated with generating the abstractions and flooding it to the VPN CE routers. However, this control overhead complexity is dependent on the granularity of the TA chosen by the VPN, and the frequency at which VPN wants the TA to be updated by the VSP.

\subsection{Thesis Contributions}

This thesis proposes a novel idea of sharing core topology information with IP-VPNs. We realize this as a topology abstraction (TA) service for the IP-VPNs where the VSP does not assume any prior traffic specifications from the VPN. This service has been studied in the context of those VPNs whose capacity requirements arrive randomly and are short lived, and therefore would want to minimize the cost by using the resource only for the time window needed.

With this brief introduction, the following are our key contributions:

1. We propose TA as a new service provided by VSPs to IP-VPNs for sharing their core network topologies and link state information in a customized manner as required by 
the VPNs. We evaluate this novel idea in the context of a managed dynamic VPN framework that allows IP-VPNs to make intelligent dynamic bandwidth requests without the need for predicting their future demands.

2. In order to generate the topology abstractions for the VPNs we propose a novel threestage TA generation process that enables fairness and service differentiation.

3. We propose three topology abstraction schemes in a decentralized mode of TA generation to address the issue of oversubscription. These abstraction schemes differ from one another with respect to degrees of aggressiveness in terms of exposing resources, which result in tradeoffs in terms of VPN call performance metrics and core network utilization.

4. Analytically, we formulate the TA generation problem as a maximum concurrent flow (MConF) problem and maximum multicommodity flow (MMCF) problem, and propose TA schemes based on approximation algorithms to address the centralized TA generation problem. To address the faimess issue encountered while applying the TA solution based on MMCF theory, we present a new online approach called the flow balancing heuristic. Furthermore, we also propose improvements to the MMCF based TA scheme for offline implementation.

We next summarize the remaining chapters in terms of its contents and the contributions made through this research. 


\subsection{Thesis Organization}

In line with the contributions stated previously, the layout of the remaining chapters of this thesis is as follows:

Chapter 2 provides the background material for this thesis and establishes the motivation for the need for a topology abstraction service for IP-VPNs. In this chapter, we discuss the various forms of IP-VPNs, its provisioning models, the various SLA parameters typically associated with an IP-VPN service definition, and the research done so far to enable dynamic VPN service. We also discuss in this chapter the notion of topology aggregation as applied in the context of hierarchical routing, and summarize the research done so far to address the various problems related to topology aggregation.

Chapter 3 discusses reachability abstraction in existing IP-VPN solutions and how TA service can be used to enable DVS for IP-VPNs. Here we also discuss how the TA service can be realized in the context of a new framework called managed dynamic VPN framework. The framework employs key building blocks as enhancements to the well known BGP/MPLS based IP-VPN solution in an attempt to realize it in a VSP's network. We also introduce the SLA parameters called TA-SLA parameters that are relevant to realize the TA service in a practical context. These parameters also serve to generate service differentiation among the VPNs subscribing to the TA service. Furthermore, this chapter discusses the various TA abstractions types considered in this thesis and illustrates how they can be realized from a fully meshed abstraction.

Chapter 4 introduces the TA generation process, for which we propose a threestage methodology that results in TA abstractions that are customized to each VPN 
depending on its negotiated TA-SLA parameters and the VSP's fairness criteria. We evaluate the different types of abstract topologies and compare their performance through simulation analysis of TA service for multiple VPNs. We also study how the TA-SLA parameters can be used to achieve service differentiation among the VPNs.

Chapter 5 defines a resource sharing problem encountered in the context of the TA service to the VPNs. In this problem, which is called the VPN topology abstraction (VPN-TA) problem, the TAs for the VPNs are independently generated in a decentralized manner by the border nodes that host them. As a solution to this problem, we propose and describe in detail three abstraction schemes; in addition, we study their performance through simulation analysis.

Chapter 6 examines the problem of generating TAs in a centralized manner. In order to study this, we define another problem called the VPN core capacity sharing (VPN-CS) problem. We then apply the multicommodity flow theory to address this problem. We describe centralized abstraction generation schemes based on maximum multicommodity flow (MMCF) and maximum concurrent flow (MConF) formulations and apply approximation algorithms to solve them in the context of the TA service for the VPNs for online implementation. We also study several enhancements of the MMCF approach so as to achieve fairness in terms of sharing of core capacity. We conclude this chapter with simulation analysis of the proposed centralized abstraction schemes and try to address the conservative nature of the centralized abstraction schemes by a technique based on oversubscription of residual link capacity. 
Chapter 7 presents the conclusions and provides recommendations on the future extensions of this research.

Appendix $\mathrm{A}$ is a reference section of the algorithms from the graph theory literature that have been used to propose algorithms for the problems studied in our research.

\subsection{Publications}

Following are the list of publications that have resulted from this research:

1. Ravi Ravindran, Peter Ashwood-Smith, Hong Zhang, and Guo-Qiang Wang, "Multiple Abstraction Schemes for Generalized Virtual Private Networks", Proceedings of IEEE CCECE'04, Vol. 1, pp. 519 - 522, May 2004, Niagara.

2. Ravi Ravindran, Changcheng Huang, and K.Thulasiraman, "Topology Aggregation as a VPN Service", Proceedings of IEEE ICC'05, Vol. 1, pp. 105 - 109, May 2005, Seoul.

3. Ravi Ravindran, Changcheng Huang, and K.Thulasiraman, "A Dynamic Managed VPN Service: Architecture and Algorithms", Proceedings of IEEE ICC'06, Vol. 2, pp. $664-669$, May 2006, Istanbul.

4. Ravi Ravindran, Changcheng Huang, and K.Thulasiraman, "A Dynamic Managed VPN Service: Capacity Sharing based on Maximum Concurrent Flow Theory", Proceedings of IEEE ICC'07, Vol. 2, pp. 211 -216, June 2007, Glasgow. 


\section{Chapter 2}

\section{IP Virtual Private Networks and Topology Aggregation for Hierarchical Routing}

\subsection{Introduction}

In this thesis, we propose a novel approach to share core topology information with the IP-VPNs. We propose the use of topology abstraction (TA) as a service to the IP-VPNs and study it under a framework that enables dynamic VPN service (DVS) to VPNs. Before conferring the details of our proposals, we present an overview of IP-VPN service in this chapter, as well as a summarization on the use of topology aggregation in the context of private network-to-network interface (PNNI) and open shortest path first (OSPF) protocols.

In Section 2.2, we begin our discussion with a brief introduction concerning the evolution of different types of IP-VPN connectivity and the SLA parameters that are used to define a managed IP-VPN service. Sections 2.3 and 2.4 discuss the two forms of IPVPN service, specifically, the customer premises equipment (CPE) and network based 
IP-VPN service. In Section 2.4, we discuss the IETF proposals for provisioning network based managed IP-VPN service. Section 2.5 summarizes the literature related to various VPN provisioning models, and Section 2.6 summarizes the research in the area of realizing dynamic VPN services. Given that the goal of this thesis is to propose the use of the notion of TA in the context of IP-VPNs, Section 2.7 discusses the application of topology aggregation in order to scale routing in the context of PNNI protocol applied in asynchronous transfer mode (ATM) networks, as well as the research that has been done to address the issues arising from applying TA to scale routing. In addition, we also discuss how TA's usage in well known link state protocols, such as OSPF, enables IP routing in large networks.

\subsection{IP Virtual Private Networks}

As defined in Chapter 1, a virtual private network (VPN) [Yua01] refers to a logical network which links entities of the same user group (enterprise, provider, etc...) that are geographically dispersed and overlaid over a shared transport network owned by a VPN service provider (VSP). A VPN is normally guarded by one or more service level agreement (SLA) attributes, which are related to control and data plane functions that guarantee a certain level of performance with respect to data security, quality of service (QoS), service availability and service pricing requirements. A VPN, whose transport connectivity over the SP's network is processed in the IP layer (i.e. uses the IP header information, like source-destination IP address and/or the TCP/UDP port information, to transport packets from one point to another) is called an IP virtual private network (IP- 
VPN), and such an IP connectivity service that is provided by a VSP is called an IP-VPN service.

The necessity for IP-VPNs came about primarily for three reasons. The first reason was to provide an interconnection between the multiple geographically dispersed sites of a given enterprise; this form of connectivity is generally known as site-to-site VPN connectivity. The second reason was attributable to the need to provide corporate intranets with access to the telecommuting users; this form of connectivity is called remote VPN connectivity. The third reason was so that to allow third parties, such as suppliers, vendors, etc..., partial or full access to an enterprise's intranet; this form of access is called the extranet service. In our research, we consider the first scenario, where sites of an enterprise require connectivity with each other.

The infrastructure of the SP over which IP-VPN is supported could either be part of the network handling Internet connectivity or a multiservice infrastructure dedicated to support the VPN service and/or other forms of managed services with certain guarantees. The difference between the two setups lies in the fact that, in the former case, the SP is unaware of any form of the VPN's existence, and hence, the traffic traversing the network is mostly afforded best-effort service. Another means of realizing such a form of IP-VPN service is when the SP only provides a point-to-point guaranteed bit rate connectivity using layer 1 technology, such as SONET/WDM or layer 2 technologies like ATM or frame relay; in this approach, the VPN themselves manage the IP layer connectivity. On the other hand, in the case of a managed IP-VPN over a multiservice network infrastructure, there is normally a well understood SLA contract between the 
VPN customer and the VSP; this contract provides certain guarantees in terms of QoS, security requirements, and service availability for the IP-VPN traffic traversing its network. In this form of IP-VPN, the framework consists of two key components. One is a set of geographically dispersed local networks or sites that belong to a particular customer, and the other is a backbone network of a VSP that interconnects these sites. Another logical extension of the customer's VPN entity is the case where the ability to remotely access the customer network may be required. Fig 2.1 gives a general view of a managed site-to-site IP-VPN service, where three sites (S1-S3) of the VPN customer are connected using a service provider's backbone. $\mathrm{R} 1$ is a remote entity requiring connectivity to the VPN.

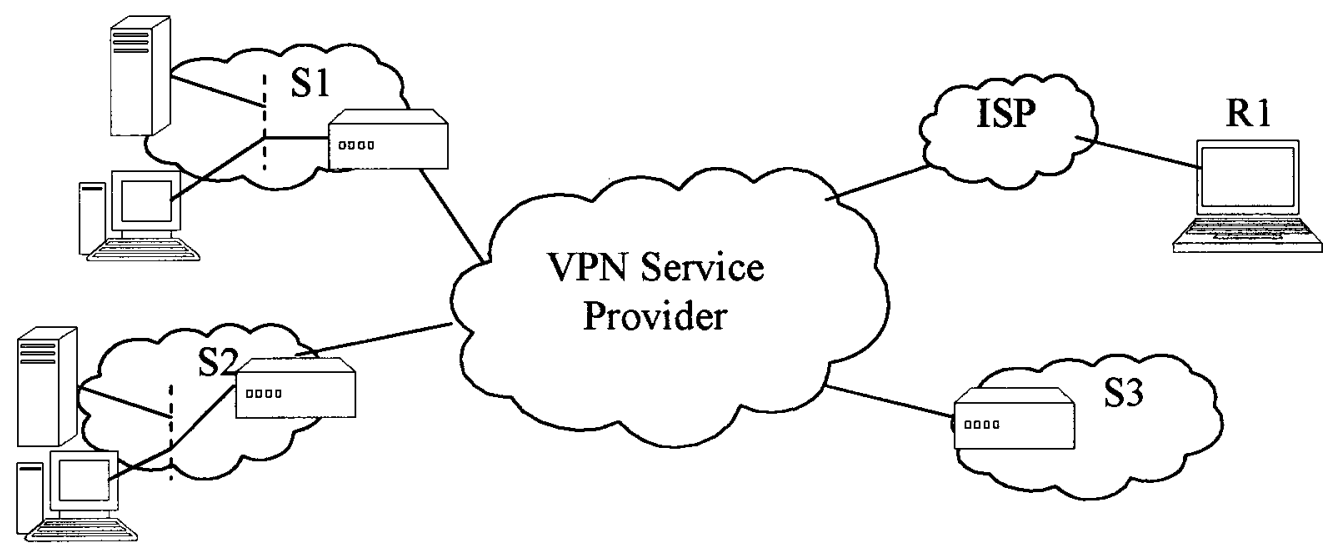

Figure 2-1: High level view of managed site-to-site IP-VPN service

Any form of managed IP-VPN is expected to provide some guarantees in terms of the data that traverse through the VSP's network, which is formalized as an SLA between the VPN and the VSP. 
At a high level, an SLA is a binding contract between the service provider and the VPN customer, and the specific set of parameters and values that define this agreement is called the service level specification (SLS). [Sar06] provides formal definitions for an SLA and an SLS and also enumerates key elements of an SLS for contemporary VPN definitions. For instance, an SLA might simply consist of the pricing for the VPN service and the exact committed bandwidth assured to the traffic traversing the VSP's network; however, with the convergence of all forms of communication, i.e voice, video and other multimedia applications, over an IP based platform, more stringent assurances need to be enforced in the transport network so as to isolate issues associated with data security, congestion, and QoS violation of flows from the VPNs. In this case, in addition to bandwidth guarantee, the SLA will also include parameters which spans VPN requirements in terms of QoS for the traffic flow in terms of end-to-end delay, jitter, and packet loss ratio requirements; service reliability guarantee; and data security requirements. We next elaborate on these different SLA categories relevant to the case of an IP-VPN service:

VPN Data Security: This is the most important requirement for any form of IP-VPN connectivity. VPN security functionality is required in order to address three common problem areas of security: authentication, confidentiality, and data integrity. Authentication ensures that the entity claiming to have sent the message is the same as the entity that actually sent the message; this is normally enabled via the utilization of digital signatures. Confidentiality ensures that the message remains private between the 
two communicating entities. Furthermore, this level of privacy requires the message text to be ciphered before being transmitted. Data Integrity requires that the message communication does not become altered during transit. Often a small digest, like the message authentication code (MAC) of the actual message, is used for this purpose. A well known security protocol suite called IP Security (IPSec), which comprises of authentication header (AH)[RFC2402] and encapsulation security payload (ESP)[RFC2406], is commonly applied in the context of IP-VPNs to enforce previously mentioned aspects of VPN data security.

VPN Traffic Isolation: As data from the VPNs are statistically multiplexed in the core of the VSP's network; there should be a mechanism to logically or physically separate data belonging to a VPN from the public or the data belonging to other VPNs. The common methodology that is used to enable this is to apply the concept of tunneling, where a protocol layer is repeated by encapsulating the original payload inside the replicated layer. In addition to hiding the original payload, tunneling also allows one to apply a common set of services at the ends of the tunnels, such as security services like the ones mentioned above.

Some of the tunneling protocols commonly used are point-to-point tunneling protocol (PPTP), layer 2 forwarding (L2F) protocol, and layer 2 tunneling protocol (L2TP). These protocols are specifically designed to tunnel point-to-point frames through an IP network commonly used for remote access applications. These tunneling protocols are also useful to transport packets from a VPN site within an IP network, 
where tunneling may be applied due to addressing or security reasons. The addressing constraints occur due to the non-routable private addresses typically used within a VPN site. Though, simple tunneling procedures like IP-in-IP tunneling take care of the addressing issue, the fact that a packet can still be read during transmission renders data security threats to the VPN, and for this reason, security enabled IP tunneling protocols like IP-Security (IPSec) are implemented.

Another form of tunneling protocol that that has been widely accepted today is the multi-protocol label switching (MPLS) protocol [RFC3031]. In an MPLS based network, a label is inserted between the IP header and the MAC header in order to transport the payload between the two end points of an MPLS enabled transport network; this can be visualized as a tunneling mechanism, since the IP payload header is not used for routing the packet. Several proposals have extended the MPLS framework as a converging technology for the next generation of layer 2 and layer 3 VPN services. In order to transport the VPN packets, a mechanism called label stacking is used to create logical separation of VPN traffic from multiple VPN customers in the transport plane.

VPN Quality of Service (QoS): Since IP-VPN services are managed by a VSP over a shared infrastructure, QoS guarantees for both real time and non-real time services are required in order to meet the end-to-end quality of experience (QoE) of a user part of a VPN site. Real time services and particularly interactive services will require stringent QoS requirements in terms of latency, jitter, packet loss, and bandwidth requirements. 
Non-real time services like web-browsing have more elastic requirements, such as page response time and average throughput.

In the context of a VPN, it is a non-trivial task to provide flow guarantees that are specific to each application. Guarantees are typically provided to the aggregate traffic from a VPN site that ingresses the core VSP's network. The aggregate traffic specification of a VPN is normally specified in terms of a traffic matrix, where an element of the matrix represents the maximum aggregate traffic flow expected between two border nodes of the VSP network hosting the VPN. This information can be used to formulate a traffic engineering problem in order to meet the VPN constraints and the VSP's objectives. Once sufficient capacity to support the aggregate traffic has been provisioned across the core, DiffServ [RFC2475] framework controls, such as packet marking, policing, shaping, and scheduling, can be applied so as to provide preferential treatment to flows emanating from a VPN.

VPN Service Availability: VPNs typically belong to enterprises or organizations that have different business requirements and priorities. In most cases today, an enterprise conducts transactions that have significant monitory implications to the enterprise over its VPN; this motivates the need for guarantees in terms of service availability. IP-VPN service availability is typically quantified by parameters such as maximum downtime that can be tolerated by a VPN during a particular duration of time, which normally is specified in terms of weeks, months, or years. These stringent assurances are typically fulfilled by the VSP by applying appropriate service availability measures to the VPN 
data traversing its networks; a commonly applied approach is to enforce route protection and switching mechanism to the tunnels transporting the VPN traffic.

From the above discussion, we can observe that an SLA forms the cornerstone of any IPVPN deployment. Enforcing SLA guarantees is a complicated procedure which spans management, control, and data planes of the transport network. The policies to enforce these service requirements are configured in routers using the VPN management systems, while incorporating VPN specific rules in the VPN security gateways and edge routers. These rules enforce VPN specific control plane signaling and data path packet processing rules; this result in enforcing the desired QoS service differentiation, data security, and service availability for the flows emanating from the VPN sites and between the VPNs themselves.

\subsection{Types of IP-VPN}

IP-VPNs can be broadly classified into two categories based on the entity managing the VPNs' control and data plane functions: CPE based IP-VPN and network based IP-VPN.

\subsubsection{CPE Based IP-VPN}

In this case, the IP layer control and data path functions related to managing the VPN site-to-site connectivity are controlled by the VPN customer. Hence, in this situation, the service provider only provides the transport layer connectivity as required by their VPN customer, and the SLA will be the bandwidth associated with virtualized connectivity 
between two sites of the VPN. These virtual circuits that connect the VPN sites are typically built on Layer 2 transport technologies, such as asynchronous transfer mode (ATM) or frame relay (FR), which enable only point-to-point virtual circuit (VC) connectivity between VPN sites as shown in Fig 2.2. The VPN customer uses these permanent VCs to manage IP connectivity between the sites. CPE based VPNs have a serious drawback, which is scaling when new sites require to be connected to the VPN. Assuming that the VPN already has $N$ sites, introducing a new $(N+1)^{\text {th }}$ site would require the enterprise to request $N$ new VCs in order to maintain a fully meshed VPN connectivity. Also, this form of configuration has an IP layer overlaid on the mesh of VCs, which leads to scalability implications to the overlaid routers that have to maintain $O(N)$ routing adjacencies. This form of VPN also requires the service provider to have the committed bandwidth circuits available at all times, irrespective of their utilization, and thereby denying the VSP the ability to multiplex the resource with other VPN users. These factors led to the proposal for the network based VPN that is discussed in the next section.

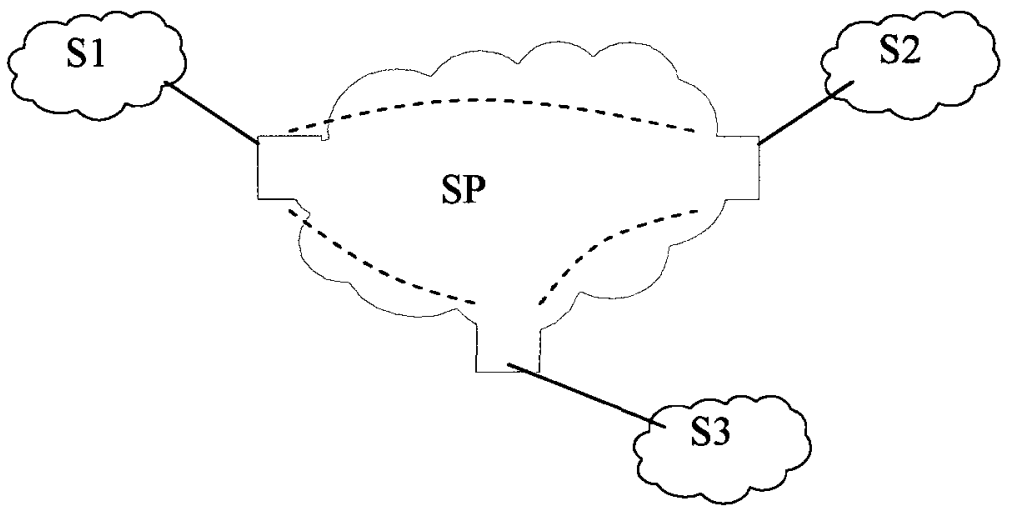

Figure 2-2: High level view of CPE based IP-VPN service 


\subsubsection{Network Based IP-VPN}

In this form of IP-VPN, the VPN customer offloads partial or full management of the VPN to the service provider. This form of service is mutually beneficial, both to the enterprise and the VSP. For the VPN customer, this form of VPN reduces the CAPEX arising from planning, provisioning, and managing its own VPN. From the point of view of the VSP, such a model of enabling IP-VPN not only offers new avenues for revenue, but also improved total network capacity utilization by enabling capacity multiplexing among the VPN customers. But this gain also comes with the complexity of meeting the VPN customer's SLA requirements in terms of the service parameters discussed in Section 2.2. An approach for a VSP to enable network based VPN is to reuse the traditional ATM or FR infrastructure, but this, as we noted in the CPE based case, leads to scalability issues. Recent drive towards convergence of multiple managed services over a single transport network has the VSPs using an IP/MPLS based transport infrastructure. A well utilized IP network not only enables it to provide seamless handling of customer's IP traffic in terms of enhanced control and data handling services, but it also makes operation, administration, and management (OAM) easier and less expensive; this is because it eliminates the need for replicated specialized network infrastructure, which used to be a typical scenario with service providers that incurred replicated CAPEX and operational expense (OPEX).

The telecom vendor community through the IETF has proposed VPN solutions where the service providers could offer managed VPN services called the provider provisioned VPN (PP-VPN) services. These managed VPN services have been defined 
for all three types of transport layers based on layers $1 / 2 / 3$ transport technologies. A summary of these VPN solutions has been presented in [Tak04][Kni04].

Layer 1 (L1) PP-VPN service provides OSI layer 1 connectivity between the VPN sites. This means that the VPN connection is identified through physical characteristics of layer 1 transport, such as a time slot in the case of a circuit switched network (e.g., synchronized optical network (SONET) technology) or wavelength switched transport infrastructure. With respect to standard activity to formalize layer 1 VPN framework and protocols, [Tak04] summarizes the efforts underway in ITU in this area, which also include features like dynamic switched connection service and customer controlled soft permanent connection specifications.

Layer 2 (L2) PP-VPN deals with providing OSI layer 2 VPN connectivity. This could be over traditional technologies like FR or ATM. More recently, providing pointto-point and point-to-multi-point ethernet connectivity on an MPLS transport framework has gained a great deal of acceptance. [Kni04] discusses the recent developments at the IETF to define L2 VPN service to transport ethernet frames between VPN customer sites on an IP/MPLS network.

Layer 3 (L3) PP-VPN, as discussed earlier, relates to managed IP-VPN service connectivity at the IP layer. [Kni04] has summarized the various proposals being considered in the IETF intended to define L3 provider provisioned VPN service. Since our research is in the context of L3 VPNs, next we discuss this service further, specifically, we elaborate on how a provider provisioned IP-VPN service is realized over a MPLS transport network. 


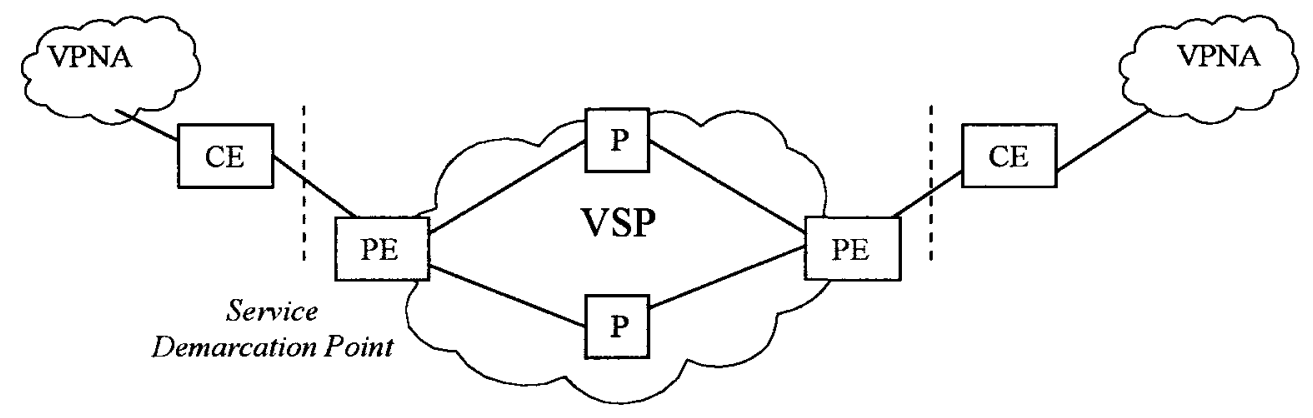

Figure 2-3: High level view of provider provisioned IP-VPN service

\subsection{Provider Provisioned IP-VPN Service}

Fig 2.3 shows the reference diagram for a L3 PP-VPN service; there are three key components to this architecture. The set of provider edge (PE) nodes, which have direct connectivity to the VPN sites, are the ones that are VPN service aware, in the sense that only these nodes in the core network has the ability to do any VPN specific operations, such as tunneling or applying VPN specific QoS policies like traffic policing, shaping, and differentiated scheduling operations. The customer edge (CE) node is part of a VPN site, and it is connected to the PE device. The link connecting the CE and PE node forms the service demarcation point for both the VPN customer site and the VSP domain. In addition to the PE node, the core provider $(\mathrm{P})$ nodes that have no connectivity to any of the CE nodes are simply high capacity switching nodes, which just switch or route data in the VSP's network in addition to taking part in the routing and signaling related to computing and provisioning tunnel paths between the PE nodes. These nodes have no intelligence to provide any VPN specific treatment to the packets traversing through it. 
The two solutions that have been proposed to realize managed L3 PP-VPN service are border gateway protocol (BGP) and MPLS based solution as proposed in [RFC4364] and virtual router based approach as suggested in [Kni06]. Though these proposals achieve the same goals as required by [RFC3809] to realize provider provisioned IP-VPN service, they take different approaches in terms achieving these objectives. The key requirements suggested in [RFC3809] are:

- Opaque transport of data between VPN sites, since the customers may be using non-IP protocols or may be using local addresses not unique to VSP's domain.

- Security of VPN data transport, so as to avoid misdirection, modification, spoofing, or snooping of customer data.

- Adherence to the SLA agreed upon between the VPN customer and the VSP, the most important being QoS guarantees to the data traffic transiting the VSP's network.

We next elaborate on the solution proposed in [RFC4364], which is of interest to us in this thesis. [RFC4364] addresses two key problems encountered in the context of the managed IP-VPN service definition: first, scaling the IP-VPN service to any number of VPN sites and minimizing the complexity of adding and removing sites from the VPN, and the second being protocol extensions intended to differentiate VPNs both in control and data plane, and a methodology to route IP flows from VPNs between geographically dispersed sites. 
To address the complexity issue, the scope of the VPN control and transport plane logic is limited only to the PE nodes. The core routers or the P nodes are ignorant about the existence of any VPN. To address the other issue of creating a logical separation between the VPNs, [RFC4364] proposed solutions whose key components include: a definition of a virtual routing and forwarding (VRF) instance, which can be considered as logical routing and forwarding instance dedicated to serve a given VPN on a PE node; extensions to BGP in order to handle the exchange of VPN specific information between the PE nodes in a unique manner; VPN aware label switched paths (LSP) to facilitate VPN data transport between the PE routers in an MPLS network; QoS to the VPN traffic; and VPN data security. We elaborate on these below.

Virtual Routing and Forwarding (VRF): One of the key requirements of a managed VPN service is the logical separation of the VPN instances in the VSP's domain. For this reason, each VPN instance hosted on a PE node is configured as a separate virtual routing and forwarding instance. The minimal configuration begins by identifying the set of CEPE physical or logical connectivity as part of the VRF instance. In addition, the VRF also maintains a database to hold service definitions specific to each VPN, such as the traffic shaping and policing aspects related to processing of the VPN packets. For a given VPN, the VRF definition on the PE nodes can also be considered as the VPN customer's routing instance in the VSP's domain, which can be configured to enforce routing policies realizing various forms of logical topologies like the fully meshed or hub-andspoke forms of site-to-site connectivity. 
Before accepting data from a VPN, each of the PE nodes is provisioned with VRF instances corresponding to the VPNs served by the PE node. To enable seamless VPN routing, a methodology has to be applied, so that the PE nodes are aware of the VPN IP addresses hosted on remote VRF instances belonging to the same VPN hosted on other PE nodes. This can be achieved by statically provisioning the VPN specific remote addresses in each PE node. This is ideal since it allows obvious control over computing resources and security, but this does not scale well as the size and complexity of a VPN increases. Alternatively, dynamic auto-discovery schemes can be adopted by piggybacking the information on one of the routing protocols used by the operator. [RFC4364] recommends the use of extended BGP, also known as multi-protocol BGP (MP-BGP), to exchange VPN specific information. The VPN site IP addresses along with qualified attributes, such as route distinguisher (RD) and route targets (RT) are used to manage the routes that are exported from and imported into a VRF.

Label Switched Path: A LSP is a label switched tunnel established in an MPLS network using well known protocols like CR-LDP [RFC3472] and RSVP-TE [RFC3473]. Allocation of labels is initiated by the PE nodes for a given forward equivalence class (FEC) that defines the class of packet properties like destination IP address, TCP/UDP port etc., which maps to a set of flows entering the provider's network through the PE node. Though the LSPs define a way to tunnel data between any two points in the network, which suffices even for a complicated service like VPN, this would result in defining multiple parallel LSPs for different VPN FECs leading to an explosion in the 
number of labels used in the network. For this reason, a two level label stack is employed. In this case, the top level label aggregates all the traffic belonging to the different VPNs between the same pair of PE nodes ingressing and egressing the VPN traffic. To transport a VPN packet, a two level label stack is used. The inner label, which is called the service label, maps to a given VPN, and hence, enables an egress PE node to identify the VPN instance and send it out on the right CE-PE link. The outer label called the transport label enables the ingress PE node to ship the packet to the egress PE identified by a route lookup on the VPN packets destination address and map it to a particular LSP.

QoS for Provider Provisioned IP-VPN: The key factor which determines how QoS is applied in a managed IP-VPN scenario depends on the granularity of LSP that connects the VPN sites. There are two ways to tunnel the VPN data. The first way is to use a mesh of tunnels for each VPN, in which case there are no competing VPNs contending for the same resource within the same tunnel. This approach has the disadvantage of provisioning and maintaining $O\left(|V P N|^{*}|P E|^{2}\right)$ tunnels in the worst case, where $|V P N|$ and $|P E|$ are the number of VPNs and the number of border nodes respectively, and so would be inefficient and costly for the VSP. The other approach is to aggregate all the VPN traffic into a single set of fully meshed $O\left(|P E|^{2}\right)$ tunnels, with the tradeoff of now sharing the core router's link and router bandwidth among the VPNs. VPN QoS in both the above cases is normally assured using a mix of IntServ [RFC1633] and DiffServ [RFC2475] service frameworks. The IntServ QoS principles are applied to traffic engineer the LSP 
tunnels using signaling features like explicit routing (ER) and constraint based route (CBR) computation solving network optimization problems, while taking into consideration aggregate VPN traffic demands. But since these explicitly provisioned tunnels serve as transport channels for aggregate traffic emanating from different VPNs, DiffServ framework of packet marking and classification at the PE edge is applied, thus classifying the VPN traffic into a pre-defined class of service $(\mathrm{CoS})$ aggregates, which statistically guarantees minimum bandwidth availability. Further, various combinations of bandwidth guaranteed tunnels and the concept of label stack mapping to desired CoS within or without VPN context can be used in the provider's network to enforce more or less granular service guarantees.

VPN Data Security: As such, MPLS provides the same kind of security as ATM and FR, since the packets are labeled as soon as they enter the VSP's MPLS network and only uses the label context to transport the packets across the network. In addition, there may also be a need to encrypt and authenticate the VPN data. [RFC4364] recommends the application of these security requirements even before the VPN traffic enters the VSP's domain using the IPSec suite of protocols [RFC2406][RFC2475].

\subsection{IP-VPN Resource Provisioning Models}

In this section, we survey the resource provisioning models proposed in the context of managed IP-VPNs. Traditional provisioning of IP-VPN was done using a site-to-site traffic estimate, also known as the PIPE model [Jut05] of provisioning. In the PIPE 
model, the traffic matrix $T=\left\{t_{i, j}\right\}$ is used to determine tunnel paths between the border node pairs, assuring the committed bandwidth guarantee for the IP-VPN traffic traversing the tunnel. The element $t_{i, j}$ is either the worst case bandwidth requirement or the estimated traffic between end points $i$ and $j$.

Given the traffic matrix, a VSP will then determine the virtual circuits that traverse the VSP network; this will be accomplished by solving the problem using the multicommodity flow formulation [Ahu93] adapted to a VPN context with business constraints and optimization objective in order to serve the VPN and VSP's interest. [Chun03] proposes a multi-objective linear optimization formulation for provisioning VPN LSPs in the context of a MPLS transport. The paper points out that a single objective function, as proposed in [Gir00], which minimizes a weighted linear sum of per-link bandwidth usage, or as in [Wan99], which minimizes the maximum link utilization of the network, addresses only one aspect of the traffic engineering problem. Hence, [Chun03] proposes a multi-objective formulation that takes into account both resource usage and link utilization. Since this formulation is NP-hard, a heuristic solution is proposed to solve the problem.

Although the PIPE model is more prevalent today, it has a few practical shortcomings. For example, as the number of VPN endpoints increases, managing the VPN traffic becomes increasingly complicated, and since each of the pipes is a dedicated circuit, there can be no multiplexing gain achieved among various committed bandwidth tunnels provisioned specifically for the VPNs. This scheme also requires the VPNs to predict their traffic requirements well ahead of time in the form of a traffic matrix. 
Estimating the traffic matrix for each pair of sites is often a demanding task. This often ends with the VPN customer estimating the worst case scenarios, resulting in unwanted expenses to the VPN and poor network utilization to the VSP.

To address the drawbacks with the PIPE model, the HOSE model was first put forward in [Duf95]. It was proposed with the goal of easing traffic specification for the VPNs. Specifically, the HOSE model tries to meet the following objectives: 1) enable flexibility to send traffic to a set of endpoints without having to specify the detailed traffic matrix; 2) reduce the capacity requirements of access links through multiplexing gains obtained from the natural aggregation of the flows between endpoints.

In case of a HOSE model, the VPN customer specifies aggregate traffic requirements for each of its end points peering with the PE nodes of the VSP. The aggregate specification for each end point $x$ is given as $\left(I_{x}, O_{x}\right)$. Here, $I_{x}$ represents the maximum traffic ingressing the end point $x$ and destined to all the other end points of the VPN. $O_{x}$ represents the maximum traffic egressing an end point into the VPN site originating from other end points. This traffic originates from all the end points of the VPN and destined to the end point $x$. [Duf95] shows that from a VPN's perspective, aggregating traffic at the hose provides significant multiplexing gain over the access link with gains in the range of $200 \%-300 \%$ in comparison to static PIPE based provisioning. Though this form of traffic specification eases the problem of traffic estimation for the VPN customers, it is a challenge for the providers to use this to provision the network, so 
as to maximize its network utilization and meet the aggregate ingress/egress bandwidth requirements of each VPN end point.

[Kum02] shows that the generic problem of provisioning the HOSE model with asymmetric $\left(I_{x}, O_{x}\right)$ specification is an NP-hard problem. For the special case in which the ingress and egress bandwidths for each VPN endpoint are equal, the authors proposed an algorithm for computing the optimal tree, whose time complexity is $O(m n)$, where $m$ and $n$ are the number of links and nodes in the network. The paper also proposed an integer programming formulation for the general VPN tree computation problem, used in cases where ingress and egress bandwidths of VPN endpoints are arbitrary, and developed an algorithm that is based on the primal-dual method.

[Jut05] studied the bandwidth efficiency of the HOSE model of provisioning versus the traditional PIPE model. One of the important conclusions of this paper was that, in the case when the HOSE model is used, over-provisioning increases with the increase in network size; however, when the uncertainty of the traffic distribution is high this is a useful model of provisioning. The study covered various HOSE model provisioning schemes that were proposed in [Duf95]. A conclusion noted was that the over-provisioning factor was less significant for tree routing based realizations. It was also observed that the average over-provisioning factor for the VPN-specific state HOSE realization increased with the topology density, and it is essentially constant for tree routing based HOSE realizations. Moreover, they observed that in the case of the VPNspecific state realization, the addition of new links to the topology does not improve bandwidth efficiency. 
Recently, [Chu08] proposed provisioning algorithms for fast VPN construction in the context of the HOSE model of provisioning under a dynamic scenario where VPNs join and leave the network. It proposed a method by which routes are pre-provisioned with the objective of maximizing the amount of admissible VPN traffic in the network. When there is a need to provision a VPN on demand, the only check for capacity is performed at the border nodes through a call admission control (CAC) process.

[Wei04] proposed a fluid VPN service model, whose objective is to address the VPN low throughput issue when the generic fluid bandwidth allocation scheme or maxmin schemes are employed in the HOSE model VPN.

Another form of traffic specification that tries to address the drawback of the PIPE model of traffic specification is called the RANGE model [Kha00]; this allows the VPN to specify the requirement as a range (lower and upper bound) of quantitative service, so that the VPNs are not required to provide deterministic point-to-point traffic estimates. This model assumes that the RANGE SLA is satisfied as long as the committed bandwidth between the two border nodes is within the agreed lower and upper bounds of the estimated traffic requirement. This enables the VSP to take advantage of the significant multiplexing gain which is achieved by managing the range based bandwidth requirements. This model is proposed over the DiffServ framework of providing QoS. 


\subsection{Dynamic IP-VPN Service}

Dynamic IP-VPN service terminology has been used to describe different characteristics related to provisioning, control, and management aspects of IP-VPNs. The properties include flexible link management schemes, the ability to add/delete/modify core resources in small time-scales, the ability to add/delete/modify the set of VPNs supported over the network dynamically, opening the control of VPNs to the end user with the ability to manipulate control, and data plane logic on demand.

One line of research in the literature is the work related to improving the core network resource utilization by managing the link capacity dynamically among VPNs. [Mit99] proposed solutions which allow dynamic resizing of the pre-established virtual circuits as a function of varying traffic demands of the VPNs, and thus improving the statistical multiplexing gain and the overall utilization of the core network. [Gar00] proposed a stochastic fair sharing scheme in order to dynamically share resources dedicated to various VPNs, while taking into account the unused capacity on each VC and sharing the capacity on the timescale of session arrivals. [Hyo03] investigated the problem of dynamically varying (increasing or decreasing) the virtual link capacity in a fair manner, and proposed a simple solution which is independent of network size or the path size of the Virtual Link (VL), and which fairly distributes the residual capacity among the competing VLs with a fast convergence time for links with varying capacity. In [Gha06], the authors studied the problem of dynamic resource management of the link resource in the context of VCAT-enabled SONET/SDH when shared by multiple VPNs. 
The paper assumes that each VPN has a provisioned guaranteed link resource and the residual non-reserved link capacity is shared among the VPNs.

Another line of research in the literature is the work related to programmable networks [Cam99] for VPNs. The objectives of a programmable VPN framework are: separation between transmission hardware and control software; availability of open programmable network interfaces; virtualization of networking infrastructure; rapid creation and deployment of new network services enabling rapid SLA negotiation; dynamic provision and modification of new or existing provisioned path's QoS properties; and the coexistence of multiple distinct network architectures. These networks are provisioned by providing VPN customers with access to a subset of the nodal and link resources controllable by the VPN and programmed based on its real time needs. The goal of this model of dynamic VPN service is to provide VPNs with the flexibility to control the provisioned switch and link capacity resource dedicated to it in the SP's network by its own control plane logic which enables it to use the committed resource judiciously.

Earlier research to realize customer controlled VPN was in the context of ATM networks [Ane99], where the VPN customer's requests are either satisfied on an existing virtual path (VP) or by creating a switched virtual circuit (SVC) between a pair of sites, and in this way enabling dynamic multiplexing of resources among different VPN sites and among various VPN customers. This paper also proposed a capacity allocation algorithm, which is intended to maximize a revenue objective as a function of VPN call arrival rates, gain and loss of revenues associated with a successful acceptance or 
rejection of calls. [Mer97] suggests a mechanism where the physical resource of a switch is partitioned. Each subset of the resource, which is called the switchlet, is presented to a different controller which could form part of different control plane architectures. [Fon02] proposed VPN resource allocation techniques in a switchlet framework using the multicommodity flow theory. [Isa00] proposed a dynamic programmable VPN architecture that allows spawning dynamic VPN networks with dedicated router and link resources over a switchlet framework. The guaranteed resource allocations made possible by switchlet partitioning ensure that the freedom afforded by opening up the control plane is contained in a safe and managed environment.

The above discussed programmable VPN architectures and solutions enabling dynamic VPN creation, assume availability of access to physical router, and link resources through open programmable interface. In practical settings, implementing the notions of a programmable VPN is not possible where strict trust issues exist, as in the case of an enterprise and a transport infrastructure owned by a commercial VSP. Also, this kind of partitioning requires prior knowledge of the demand matrix of the VPN, prior to mapping the appropriate switch and link capacity to support the VPN.

Network based managed IP-VPN services paradigm can be exploited by the VSP in order to generate novel revenue generating services. Also, since the VPN traffic is becoming increasingly IP based, there is a good case for the VSP to maintain a single IP/MPLS network to provide traditional VPN services, such as emulated virtual leased line services with static requirements, managed IP-VPN service based on solution proposed in [RFC4364], and also more enriched dynamic VPN service to serve VPNs, 
which may require resources for a short window of time. The basic goal of our research is to propose a topology abstraction service framework that shall enable a VSP to share its sensitive core topology and link state information with the IP-VPNs in a practical and scalable manner. Another feature enabled by this service is that it enables VPNs to make intelligent bandwidth requests without the need of predicting their future demands. This prevents poor network utilization because of committed resources and enables statistical multiplexing of VPN demands in the core of the VSP's network. As the bandwidth demands of the user is not known in advance, the VSP doesn't provision any resource before hand, instead uses the notion of topology abstraction to share topology and link state information applying negotiated SLA metrics and fairness policies with the VPNs. The VPNs can use this information to request resources more intellegintly in future. Topology abstraction also allows a VSP to provide service differentiation from its topology information sharing service which also enable the VPNs to negotiate a service definition that meets its requirements.

Topology aggregation has been used before in the context of routing in packet switched networks. Our use of topology abstraction ${ }^{1}$ (TA) service to enable managed DVS for IP-VPNs is yet another application of topology aggregation. Next, we provide an introduction of the use of topology aggregation and literature survey of the problem encountered by its use in the context of routing.

\footnotetext{
${ }^{1}$ We use the term "topology abstraction" instead of "topology aggregation" in the context of IP-VPN to emphasize the fact that topology aggregation in the context of IP-VPNs is used to share the VSP's sensitive core topology information with the VPNs as a service rather than to scale routing in large networks.
} 


\subsection{Topology Aggregation for Routing}

Routing refers to a per-hop decision making process in a layer 3 domain involving a router to determine the next-hop adjacent node to which the packet should be forwarded to so that it reaches its desired destination. In the case of IP routing, the routing decision is made using the destination IP address, along with a routing table lookup. Routing in IP networks can be either static or dynamic. Static routing involves provisioning the routes (i.e. the adjacent router information for a set of IP addresses) in the routers of the network by an operator. The routes may be computed either using an offline mechanism or based on certain administrative policies so as to achieve certain traffic engineering objectives. Dynamic routing involves the application of a routing protocol in order to exchange neighbor information to build partial or complete database of the core network topology. The topology information is then used to compute the next-hop information for each routable IP address.

Our interest in this thesis is in the context of dynamic routing which we discuss further. As mentioned above, dynamic routing is enabled using a routing protocol, which has three main functions: 1) exchanging routing information; 2) managing the routing database; 3) path computation and its management using the routing database.

Exchanging routing information involves routers encoding best next-hop information for a set of routable IP destinations along with link connectivity neighborhood information in standard format as required by the routing protocol and sharing it with its neighbors. The neighbor nodes decode and apply the routing information into their own routing database and share this information with their 
neighbors if necessary. A routing database in a router manages the information received from its neighboring routers by comparing its current knowledge of topology and advertised destination information with the already installed routes, and making additions, modifications, or deletions to its database if necessary. Path computation involves using the local information available in the routing database in order to determine the best next-hop to the router which is the gateway to a destination IP address.

Depending on the type of routing information that is exchanged, routing protocols can be categorized into two types: distance vector routing (DVR) or link state routing (LSR) [Meh07].

DVR involves the routers exchanging their neighborhood and reachable IP destination information with their neighbors. The neighboring nodes use the information to determine the best route to a given destination using a path computation algorithm, such as the Bellman Ford [Ahu93] algorithm. Although DVR has the advantage of computational simplicity in determining the shortest path to all the nodes in a domain, it has the disadvantage of reacting slowly to node or link failures; this results in high convergence time complexity of synchronizing the change across the network domain. This could lead to excessive packet loss of user flows during the convergence duration.

Link state routing on the other hand is based on a reliable flooding mechanism within a domain that enables each router to build a map of the core network topology. This knowledge of the core topology is used to compute the shortest path tree locally in the router using algorithms such as Dijkstra's shortest path algorithm [Ahu93]. The shortest path tree is computed by applying optimizing metrics like cost, hop count, delay, 
or bandwidth. Given that the link state management of a routing database is based on flooding mechanism, LSR based routing protocols have better convergence time during node or link failures.

One of the design objectives of a routing protocol is for it to scale both in terms of memory and computational complexity of calculating optimal paths with increase in size of the network. The increase in the network size is a result of either adding new nodes or links to address issues like increase in traffic from the existing customers or could be also to address the need to provide path protection features to the existing set of traffic engineered paths. Increase in the size of the routing database also increases protocol overhead in terms of message size, resulting from increased number of nodes, links and destination IP addresses. A solution that has been applied in the context of routing to handle large networks is the notion of hierarchical routing applying the notion of topology aggregation. Hierarchical routing involves administrative grouping of nodes of a domain; this creates several subsets of routers of the core network called the subdomains. The subdomains can be further split to create more subdomains. Such a structural breakup of the core network can be represented by a hierarchical tree.

Routing protocols have been extended to route in domains that have been broken into a hierarchical tree. In such a form of routing, routing within a subdomain is flat, i.e. each router in the subdomain has complete knowledge of the subdomain topology it is a part of. Routing between subdomains is enabled through the process of information aggregation, wherein certain representative nodes of the subdomain summarize the subdomain's topology and link state QoS information and share them with the border 
nodes, which then floods the information across their respective subdomain. Here, the application of topology aggregation abstracts the network topology information pertaining to a domain as well as the connectivity between two domains.

A network domain can be abstracted using several forms of abstract topologies of varying granularity. This form of abstraction could be as trivial as the case where the virtual link in the abstract topology represents the connectivity between two points, and in a more complex case, it is associated with some information capturing the link state of the underlying physical network. In the context of link state abstraction, the metrics of interest include both convex metrics like bandwidth and additive metrics like link cost or delay. A metric can also be classified based on its time varying properties, such as static or dynamic. A static metric does not vary with time, or may vary at very large time scale; an example of this is hop count or link cost associated with the cost of packets traversing the link. A dynamic metrics like, link latency (due to queuing delay) and available bandwidth of a link, varies at short time scales depending on network state properties like the load and congestion status of the network. The choice of a metric, static, or dynamic, associated with a virtual link in a aggregated topology has a consequence on the protocol overhead incurred in keeping the link state information in sync with other subdomains. In addition to the topology and link state information, another important abstraction is with respect to the rechability of hosts and nodes in a subdomain.

Hierarchical routing has its own benefits and drawbacks. It has the advantage of achieving significant savings in terms of memory and computational complexity. It also enables a certain level of security because of its ability to disable topology information 
sharing across all the nodes of a network domain. But hierarchical routing could also lead to inefficient routing, since the routing decisions may not be optimal, since they are based on aggregated information. The routing efficiency in the case of hierarchical routing is highly influenced by the granularity at which the core topology is abstracted, and the correctness of the abstracted link state information.

Two well known routing protocols that have applied topology aggregation in order to achieve routing scalability are private network-to-network interface (PNNI) [PNNI96], developed for ATM networks, and open shortest path first (OSPF) [OSPF98] for IP networks. We next briefly discuss the use of hierarchical routing in these protocols.

\subsubsection{Private Network-to-Network Interface (PNNI)}

\section{Protocol}

PNNI is a routing and signaling protocol applied in ATM networks. In order to scale routing in ATM networks to a large number of nodes, hierarchical routing has been exploited. At the lowest level, switches with direct physical connectivity are clustered together by administrative configuration to form a peer group (PG). Representative nodes of a PG, which is called the designate node, summarize the peer group's topology and link state information representing it as a logical group node (LGN) at a higher level. The LGN at a higher level can further be clustered to form a PG and further abstracted. The number of levels of abstraction depends on the routing scalability requirements of a provider network. PNNI allows such a hierarchical aggregation to scale up to 104 levels. 
In the simplest form, an LGN is summarized as a complex-node, which is a simple node enabling one value aggregation per QoS parameter. This representation is conveyed in the form of a symmetric-star topology [PNNI96] that consists of a central node virtualizing the network and ports that map to the border nodes of a PG. Fig. 2.4 illustrates PNNI abstraction on a 24 node network topology. Fig. 2.5 shows the logical representation of the 4 level tree hierarchy that is enabled by the clustering of nodes in the topology shown in Fig. 2.4 .

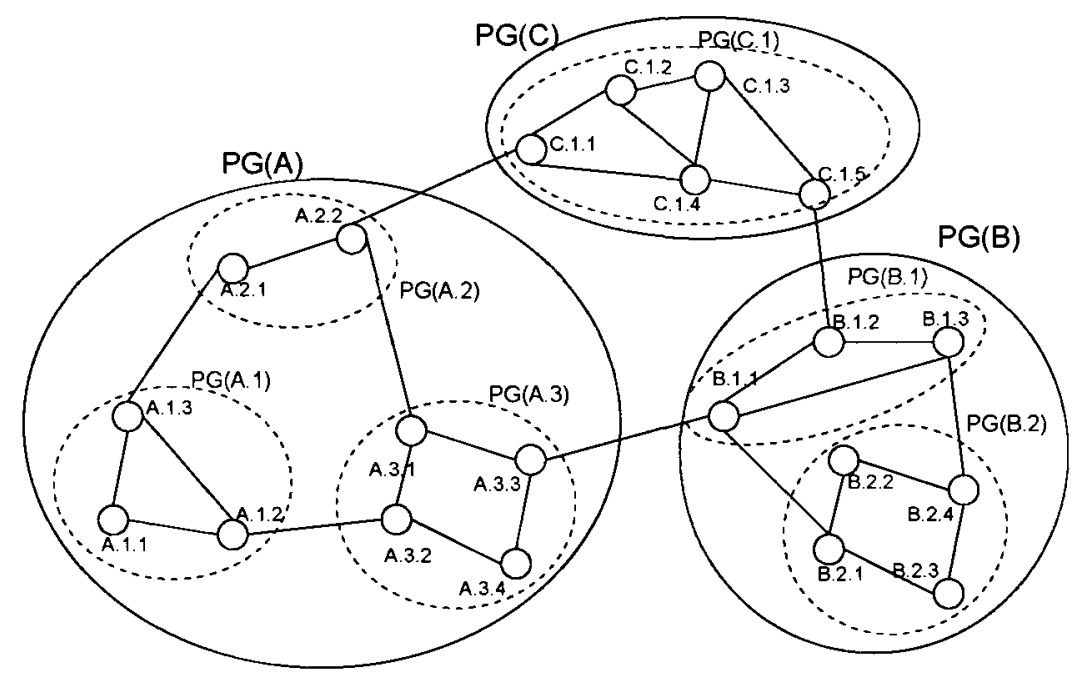

Figure 2-4: Example for PNNI topology aggregation

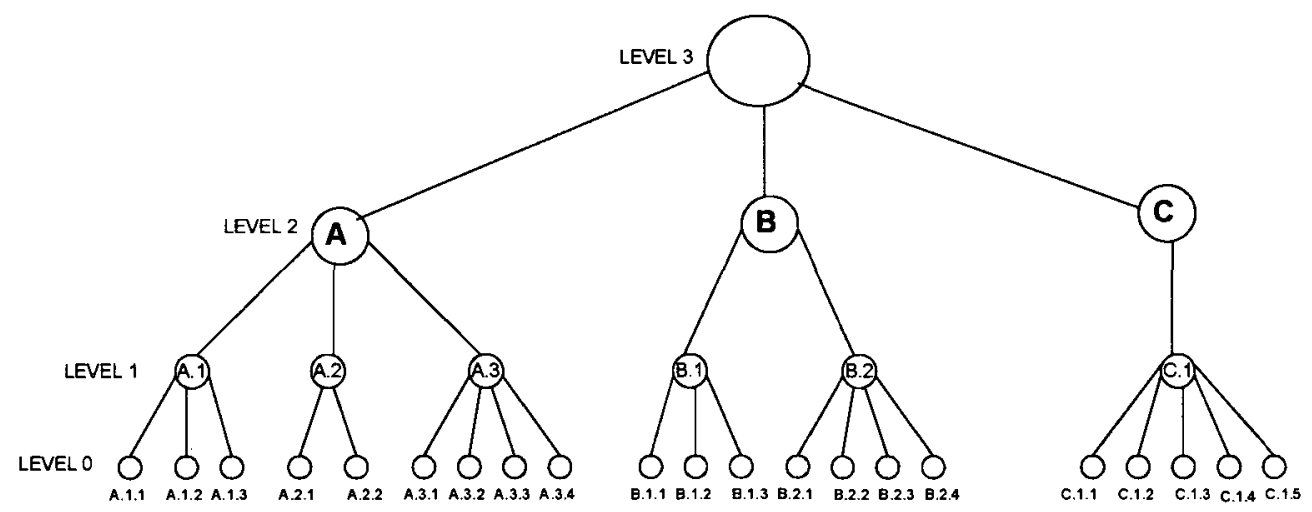

Figure 2-5: PNNI multi-level hierarchical topology aggregation 
Researchers have explored various problems related to topology aggregation in the context of the PNNI protocol for ATM networks. The problem of topology aggregation can be broken down into two sub-problems. One is to choose the appropriate abstract topology type in order to strike a balance between loss of network connectivity information and associated control overhead. The other sub-problem is with the aggregation algorithm used to associate one or more QoS link metric information with the edges of the abstracted topology with the objective of minimizing the error between the approximated and real time physical layer link state of the network.

Various forms of topology aggregation can be used in hierarchical routing, with different levels of granularity in terms of connectivity between the border nodes of a subdomain. The aggregated topology types can be as simple as representing the entire subdomain as a simple node or can be broadcasted as a fully meshed abstract topology. The simple node is the simplest of all the types, and it is best in terms of cost of aggregation, which is typically measured in terms of the size of the link state update packets. But it lacks the ability to reflect any asymmetric topology information. The other extreme of aggregation is to use a fully meshed aggregation topology, wherein the nodes of the aggregated topology map to the border nodes and the logical links abstract the physical path properties of the internal network being abstracted. This approach is preferred because of its non-lossy QoS metric aggregation; however, it suffers from scalability in terms of the topology complexity and the associated link state updates. [PNNI96] also proposed a compromising aggregation which is called the star topology. A star topology uses a pseudo node as the hub node with the border nodes as its spokes. Several other 
forms of aggregations have been proposed, between the simple node at one end and the fully meshed topology at the other end, in order to achieve certain desirable properties such as capturing information like asymmetry in QoS information while containing aggregated topology complexity. [Lee01] proposed a spanning tree aggregation connectivity between the border nodes. [Kor00] proposed a new form of star topology called the source-oriented star aggregation. Here, the central node of the star is not a pseudo node, but it is the border node that generates the topology aggregation. In this case, the objective is to provide the border nodes only a subset of the fully meshed abstract topology that is useful for computation of paths to the border nodes of the adjoining domains.

The problems of link metric aggregation can be classified into two categories: single metric aggregation and multiple metric aggregation. With respect to abstracting link metric information, the objective of topology aggregation is to provide compact topologies, while minimizing the errors occurring due to aggregation of the desired link metric information. Approaches that have tried to address the single metric aggregation problem, which has been shown to be fairly easy to solve, include algorithms proposed in [Lee95], [Lee99], and [Lee01]. These papers basically suggested optimization to the fully meshed representation associated for both additive and non-additive link metric by encoding it further to auxiliary graphs such as a minimum spanning tree and a minimum equivalent sub-spanner graph. [Lee95] also shows the difficulty with dealing with asymmetric networks, even with non-additive parameters like bandwidth. [Gue97] discusses the effect of aggregation on routing of connections with bandwidth 
requirements and suggests using a shortest path algorithm with weights proportional to the probability that links could satisfy the bandwidth requirement.

With respect to multiple metric aggregation, the non-triviality of the aggregation of the multiple metric information arises, due to the fact that it is difficult to capture the region of feasibility with a minimal set of parameters in the multi-dimensional QoS metric plane. If we consider two metrics like delay and bandwidth, this region of feasibility is bounded by a staircase region defined by convex-corner points. The problem here is to minimize the difference between the region represented by the aggregation scheme and the actual area of feasibility while minimizing the space complexity required to share this information with other domains. Many researchers have studied this problem and proposed ways to approximate the service region of feasibility. [Kor00] proposed a technique using a combination of minimum delay min_d, maximum bandwidth max_bw, and the smallest stretch factor min_s among all paths between the border nodes; here the delay-bandwidth feasible region is abstracted as a hyperbolic curve. The requests that lie in this region are accepted by the domain for signaling. The authors in [Lui04] proposed to represent the region of feasibility by a line segment instead of a single point. The line segment is determined by the least square method that minimizes the least square error, i.e. the summation of squares of distances from the corner points of the feasible region to the approximated line. [Tan05] proposed the use of service curves based on polynomial curves, piecewise cubic splines, and polylines, which were shown to be more accurate than those in [Kor00] and [Lui04] but with a tradeoff in terms of space complexity. 
One of the basic problems of topology aggregation in real time is the error in aggregated graph compared to the original topology. To remedy this, some researchers proposed new aggregation schemes that minimize the average distortion in a least square sense [Awe98]. In [Hao00], the authors proposed two topology aggregation approaches. One of them (called the hybrid approach) advertises relatively static information (e.g., hop count) less frequently, while advertising highly dynamic information (e.g., bandwidth) more frequently and in less detail. The other approach determines the most used paths based on statistical observation and gives more weight to these paths during the next topology aggregation computation cycle.

\subsubsection{Open Shortest Path First (OSPF) Protocol}

OSPF is a well known link state IP routing protocol, which enables only two levels of hierarchical routing. The protocol provides a basic feature where a core network also known as an autonomous system (AS) is divided into subdomains called 'Areas'. The hierarchical routing enabled by OSPF can be explained at a high level referring to Fig. 2.6. Each area is identified by a unique id. Area 0 is called the backbone area, and the protocol mandates other areas of the AS to be connected to it. A router is designated as being in a particular area if at least one of its interfaces is configured to be in that area. Hence, a router could also belong to multiple areas. Areas that do not have a direct physical connectivity with the backbone area are connected using virtual links (which is an IP tunnel), as shown in the case of Area 3 and Area 0 as a dashed line in Fig. 2.6. The 
border nodes of the areas summarize the IP address information along with the 'Best' ${ }^{2}$ cost of reaching the IP address in or reachable through the area and floods it to Area 0 through the area border routers $(A B R)$ that are also a part of Area 0 . The aggregate reachability information is further flooded by Area 0 ABRs into other areas as well. The path cost associated with the reachability information will be updated at each ABR before it is flooded into other areas. Since Area 0 is connected to all other areas, the information of these IP addresses, along with the path cost summary, also gets flooded into the areas that are directly or indirectly connected to Area 0 through a virtual link.

Though hierarchical routing in OSPF is not as complex as PNNI in terms of topology aggregating properties, it does play a significant role in improving the memory and processing complexity requirement of router nodes in the core network which enables operators to expand their AS to large number of nodes [Meh07].

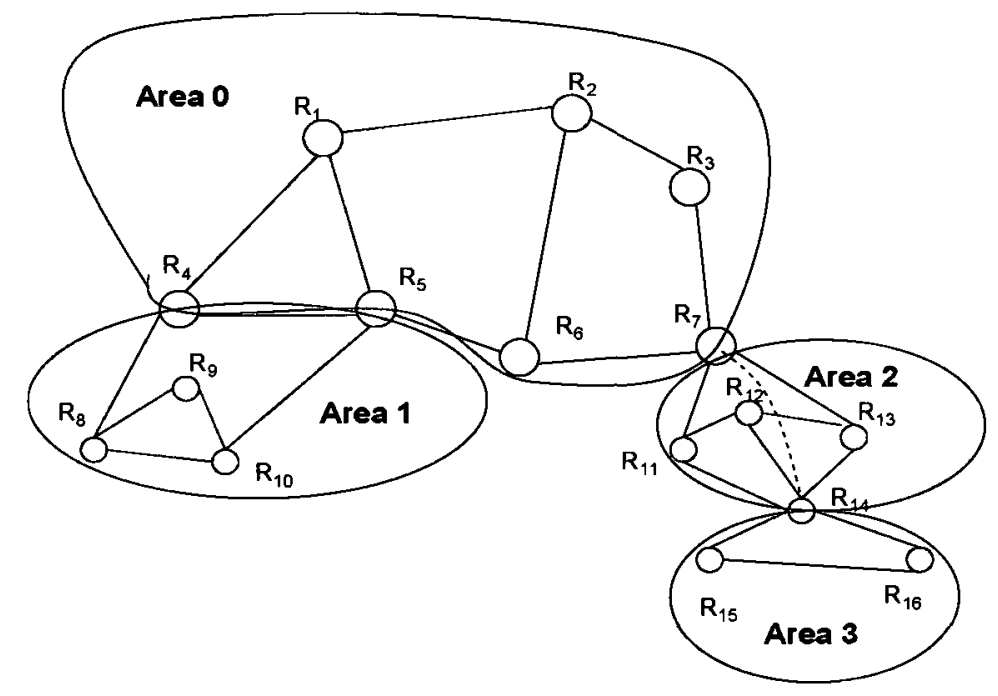

Figure 2-6: Example of hierarchical routing in OSPF

\footnotetext{
2 'Best' here refers to a path computed with an optimization objective within an area of being the shortest in terms of cost or latency.
} 


\subsection{Summary}

This chapter discussed the various concepts related to this thesis. We began our discussion with a brief introduction to the evolution of different types of IP-VPN connectivity, and the SLA parameters that are used to define a managed IP-VPN service today. In this context, we discussed two forms of IP-VPNs, which are the CPE and network based IP-VPNs. We also discussed the IETF proposed solutions for provisioning network based managed IP-VPN service. VPN resource provisioning is one of the most critical problems that need to be solved to meet QoS requirements of IP-VPN customers and maximize core network resource utilization. In this context, we summarized the literature related to various IP-VPN resource provisioning models, along with the benefits and drawbacks of applying each one of them. Since the goal of this thesis is to introduce the application of topology aggregation in the context of IP-VPNs, we discussed how topology aggregation has been used to realize hierarchical routing in the context of PNNI protocol for ATM networks, and the research that has been done to address various problems arising due to aggregation of network topology and link state information. Further, we also discussed how topology aggregation enables IP routing to scale in well known link state protocols, such as OSPF. This thesis also proposes a framework to study the use of TA for the IP-VPNs; the framework enables dynamic bandwidth service to the IP-VPNs. Therefore, we also summarized the research in the area of realizing dynamic VPN service for IP-VPNs. 
Having provided a background to the key concepts that will be employed in this thesis; we next proceed to discuss each one of our contributions in the following chapters of this thesis. 


\section{Chapter 3 \\ Topology Abstraction Service for IP- VPNs: Definition and Realization}

\subsection{Introduction}

In this chapter, we introduce topology abstraction service in the context of IP-VPNs. In Section 3.2 , we begin by discussing the applicability of the notion of reachability abstraction in current network based IP-VPN solutions. Section 3.3 discusses the need for TA for IP-VPNs; its objectives as a service; and the key differences from its use for hierarchical routing. In Section 3.4, we discuss the managed dynamic VPN framework (MDVF) along with the key building blocks that enhance the well known BGP/MPLS based IP-VPN solution to realize the TA service for the VPNs in a VSP's network. Section 3.5 introduces the SLA parameters called TA-SLA parameters that are relevant in order to realize TA service in a practical context. Section 3.6 introduces the important graph theory notations in the context of the TA service to the VPNs. Section 3.7 introduces the different TA types that have been considered in this thesis. 


\subsection{Reachability Abstraction in Managed IP-VPN Service}

Before we discuss the applicability of TA in the context of managed IP-VPNs, we discuss how abstraction is used implicitly by the VSP as a way to share reachability information between the sites of a VPN in current well known provider provisioned IP-VPN solutions [RFC4364] and [Kni06].

[RFC4364] and [Pual06] (also summarized in Section 2.4) solutions do not discuss rechability abstraction as an explicit network function; however, if one were to look closely, rechability abstraction is the underlying principle that enables Layer 3 connectivity between the VPN sites. This is enabled by instantiating appropriate routing protocol and exchanging VPN specific information between the CE and PE nodes. To begin with, the PE node first populates its own VRF instance by exchanging VPN routing information with other PE nodes hosting the same VPN; an extended version of interior border gateway protocol (I-BGP) called multiprotocol I-BGP [RFC2858] is used for this purpose. [RFC4364] also proposed a policy driven VPN route exchange, which is based on a filtering mechanism over the VPN routes exchanged and installed in the VRF that enables realization of various logical reachability topology, such as fully mesh and huband-spoke form of connectivity. Once the VRFs are populated, a routing protocol, which is supported by both the CE and the PE nodes (for example, OSPF based interaction as proposed in [RFC4577] or exterior BGP (E-BGP) as suggested in [RFC4364]) is applied between the two nodes. This enables the transfer of the remote VPN reachability 
information from the VRF database in the PE node to the CE node. This results in the CE node visualizing the peering PE node as an adjacent router or gateway to all the remote routable IP addresses of the VPN.

Fig. 3.1(a) illustrates this form reachability abstraction; the figure shows a VSP providing managed IP-VPN service to two VPN customers, VPN-A and VPN-B. The four VPN-B sites are shown to host subnetworks in the range of 10.1.0.0/16-10.4.0.0/16. Once the VRFs are instantiated in each of the PE nodes, the routing protocols exchange and synchronize all the VRFs with the reachability information relevant to VPN-B. This results in a fully meshed connectivity in terms of reachability from a PE node's perspective as shown in Fig. 3.1(b). This allows the packets ingressing at any of the PE nodes from a CE node to be correctly transported to the intended remote VPN site. When the PE node further shares this reachability information with the CE node, the CE node's visualization of abstraction of the VPN connectivity is shown in Fig. 3.1(c).

It should be noted that the edges shown in Fig. 3.1(b-c) signify the rechability property and do not have any TA related topology or link state properties. This is because the current solutions do not enable such an exchange of topological information between the VSP and the VPNs. 

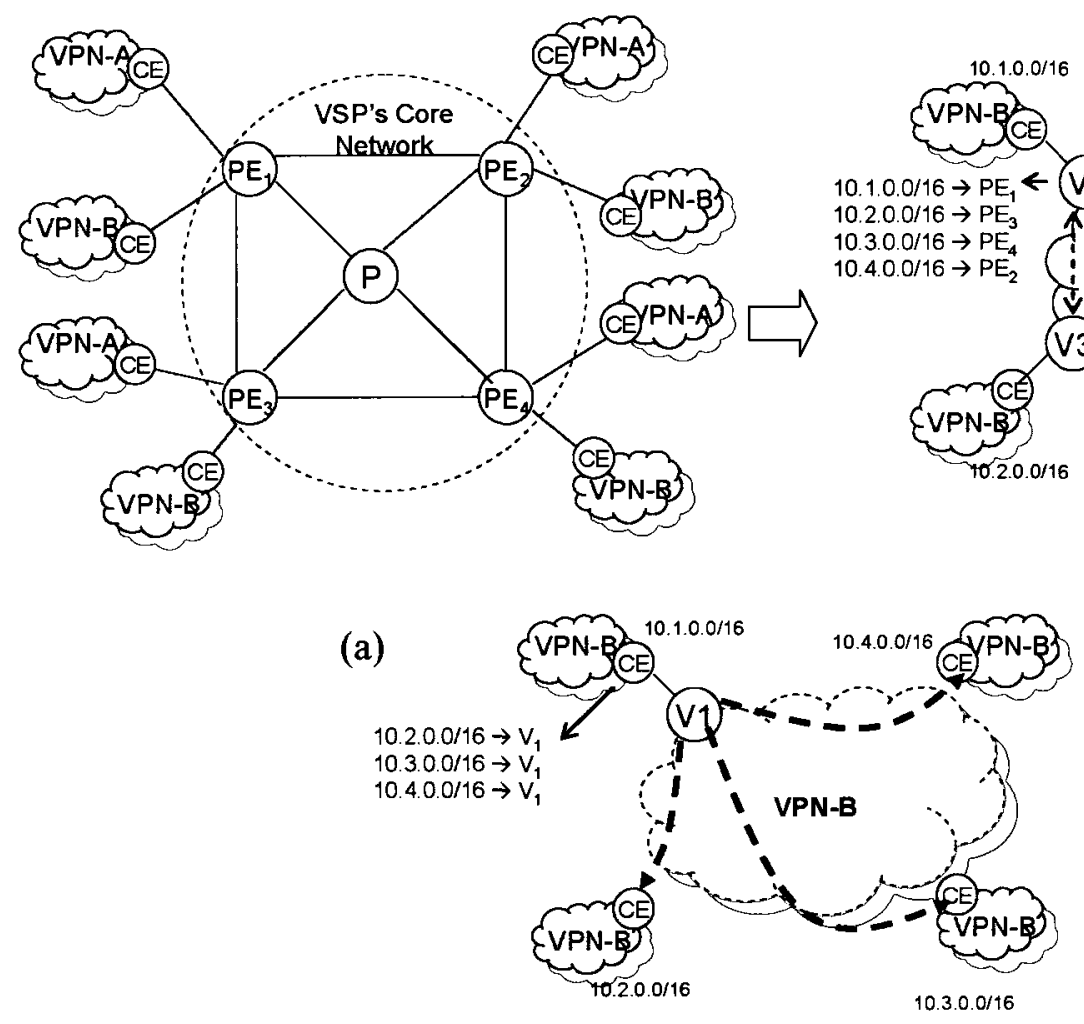

(c)

\subsection{Topology Abstraction Service for IP-VPNs}

As discussed previously, a VSP today only has the ability to disseminate reachability information to all the CE nodes of a given VPN. This information suffices the need of most of the IP-VPNs today; this is so as the QoS for the VPN traffic is pre-negotiated with the VSP, using the PIPE or HOSE mode of traffic specification and guaranteed by provisioning appropriate core resources to handle the traffic. For enterprises today, these traffic specifications are expected to only vary in long timescales. Such long time scale 
variations give a VPN sufficient time to renegotiate the existing SLA to include the future capacity requirements. Hence, the need for TA is not compelling under such static or long term varying traffic requirements; however, with the increasing number of multimedia applications in enterprises used to support applications involving conferencing, collaboration, and data management involving backups and restoration, the VPN customers, in the future, may require significant capacity for a number of small windows of time in order to meet the needs of these bandwidth intensive applications. A good example in an enterprise case is an application called Telepresence [CISCO], which involves setting up a high definition audio-video conferencing session between VPN sites. In such situations, the current approach of going through the process of renegotiating SLAs is not an acceptable one. Even if the VPN customers were able to predict the worst case capacity requirements and renegotiate the existing SLA, this solution has two unwanted drawbacks. Firstly, the VPN will have to pay for the capacity, even if it does not use it beyond the intended period of use. Secondly, the VSP who commits the resource for a given VPN could experience poor network utilization and possible loss of revenues. There are two possible, yet extreme, solutions to address these problems over current existing solutions [RFC4364] [Kni06] to provision managed IPVPN services.

The first solution is to allow a VPN to request bandwidth whenever there is a bandwidth requirement and to have the VSP apply its own CAC policies so as to determine if such a request can be accepted. Theoretically, a VPN can seek bandwidth on demand from the VSP today using current protocol constructs like LDP [RFC3472] or 
RSVP-TE [RFC3473], which enables VPNs to seek capacity on demand dynamically; however, there is a drawback with this solution. The VPN in this case has to make the request without having any information about what the VSP's network can offer in terms of its QoS guarantees during the time of request. This could lead to highly unsatisfactory VPN call performance.

The second extreme solution is for the VSP to share its core topology information with the VPNs and let the VPNs decide how to route their capacity requests. This solution may not be practical because of two reasons: First, the approach goes against the interest of the VSP who would not want to share its sensitive core topology information with its customers. Secondly, even if it does share this information, the complexity of sharing the topology information and having it synchronized with the clients' routing database would be overwhelming. Another important drawback with both the above solutions is that it doesn't provide the VSP a means to monetize this service or provide any justification to differentiate between requests from different VPNs.

In order to address this situation we take the intermediate approach that leverages the notion of TA as the key building block to enable DVS for the VPNs.

Applying TA to share core resource status with the VPNs allows it to improve its decision on availability of resources in a dynamic call scenario, which is expected to yield good probability of success in establishing a call successfully or rejecting it locally. For the VSP, the TA can be used as a tool to allow it to abstract its core network to any degree of granularity, correctness, and associate it with one or many aggregated QoS metric information it desires. This property of TA allows the VSP to negotiate the 
properties of the TA provided to the VPNs as SLA parameters and realize it as a revenue generating service. One important requirement of this service is to have the ability to realize it as an extension to the existing managed IP-VPN solutions like [RFC4364] [Kni06], and thereby, minimize the changes to the control and transport plane logic and the cost of realizing this service in current IP-VPN solutions.

Based on the above discussion, we summarize the objectives of the TA service definition for the IP-VPNs as follows:

- One that enables VSP to share core topology information as a service with appropriate SLA definition to enable service differentiation.

- The service should not require the VPNs to predict capacity requirements ahead of time.

- The service should enable the VPNs to use the TA information and perform intelligent route computation, and which can be leveraged to seek resources on demand in order to meet its QoS requirements for the duration of the call request.

- In a dynamic call request scenario, the service should also result in good VPN call performance and maximize VSP's core network utilization.

We next discuss the differences in using TA as a service to IP-VPNs versus the use of topology aggregation for hierarchical routing.

\subsubsection{Differences from Hierarchical Routing}

As we saw in the previous chapter, topology aggregation has been used to enable hierarchical routing in IP and ATM networks. We use TA in IP-VPNs in order to share 
the VSP's sensitive core network properties with the VPNs. Applying this notion in the context of IP-VPNs can be used as a service because TAs can be generated with different topology granularities and associated link state metric information. Besides this, there are several notable differences in terms of the objectives for which it is proposed in the IPVPN context and its current use for hierarchical routing which we discuss next.

Goals of Topology Abstraction Service: In the case of hierarchical routing, the goal of topology aggregation is to reduce the routing overhead associated with large networks by reducing the topology and link state information that is required to be stored by the routers in the network by imposing a hierarchical routing structure over the entire network. In the case of TA service to IP-VPNs, we use this concept to enable core topology and link state information sharing service, wherein the VSP provides the VPNs an abstracted version of the topology and link state QoS metrics information so that the VPNs can make intelligent resource requests to the VSP.

Choice of Abstract Topology: In the context of hierarchical routing, the type of topology aggregation employed to summarize and share the topology and link state information is generally homogenous throughout the administrative domain. In our case, the core topology could be abstracted as different abstract topologies for different VPN customers in order to enable service differentiation. The choice of the abstract topology to be provided to a VPN is based on the SLA parameters (discussed in Section 3.6) negotiated between the VSP and a VPN customer. 
Challenges of providing TA service to IP-VPNs: In the case of routing, the challenging problem encountered during topology aggregation is related to minimizing complexity of aggregated link state update information, and ways to mitigate information loss arising out of summarizing topology and QoS link metrics. In the case of using the TA service to enable DVS, several other problems need to be addressed, such as: fairness of exposed information (discussed in Chapter 4), resource oversubscription (discussed in Chapter 5), and maximize usage of core network resources. To address these problems, a process to generate the TAs for the VPNs which applies special TA generating algorithms is required. Chapter 5 and Chapter 6 propose several abstraction schemes applicable in decentralized and centralized mode of TA generation.

Route Computation and Path Signalling Process: In a hierarchical routing context, domains use the abstracted information in order to compute end-to-end paths. The path is further resolved when the message arrives at each of the intermediate border nodes of the neighbouring domain. In the TA service context, the abstract topology is primarily used to verify the feasibility (or availability) of its QoS requirements; this is accomplished by executing a route computation algorithm using the abstracted topology by the VPN CE node. If it perceives the path to be feasible, it signals the capacity request to the PE node, which will then apply SLA and CAC policies defined for that VPN before signalling the path to the remote $\mathrm{CE}$ destination. The destination $\mathrm{CE}$ information from the call request message would then be used by the PE node to signal a new connection in order to satisfy the VPN's request. 


\subsection{Managed Dynamic VPN Framework}

To study the use of TA service by the VPNs, we propose to realize it in the context of a challenging environment called as the managed dynamic VPN framework (MDVF). The framework tries to achieve the objectives of the TA service by realizing it over an existing IP-VPN solution, which enables its implementation in a VSP's network; the framework provides the following functions:

- Allows TA-SLA parameter (discussed in Section 3.6) negotiation between the VSP and the VPN that are relevant to the TA service for the VPNs. These SLA parameters are negotiated by the VPN based on the correctness of core topology information required at any instant of time and the criticality of applications for which it intends to use the TA service.

- Generate topology abstraction graphs to expose the available capacity in a fair manner complying with the negotiated SLA parameters and VSP's fairness policies, to enable the VPNs to improve its route computing performance and VSP to maximize its core network utilization.

- Allows the VPNs to use the abstracted topology information to seek services on demand from the VSP.

- The framework facilitates in realizing the TA service with minimal changes to an existing managed IP-VPN solution, and re-uses the well known routing protocols between the $\mathrm{CE}$ and the PE nodes to flood the topology abstraction information from the PE to the VPN's CE nodes. 
Next, we introduce the MDVF for implementation in a VSP's network which realizes the previously mentioned functions as part of the TA service to the VPNs. In particular, we propose extensions to a well known IP-VPN solution [RFC4364] in order to realize it. [RFC4364] basically uses extended I-BGP as part of the control plane and MPLS based transport plane to enable L3 IP-VPN service. We add new functional components to this solution to realize the MDVF for the VSP. Fig. 3.2 shows the components of our framework. The VPN's and the VSP's network is assumed to be controlled by an MPLS based control and transport plane. The two main boxes in Fig. 3.2 represent the $\mathrm{CE}$ and the PE node with their control and service plane components.

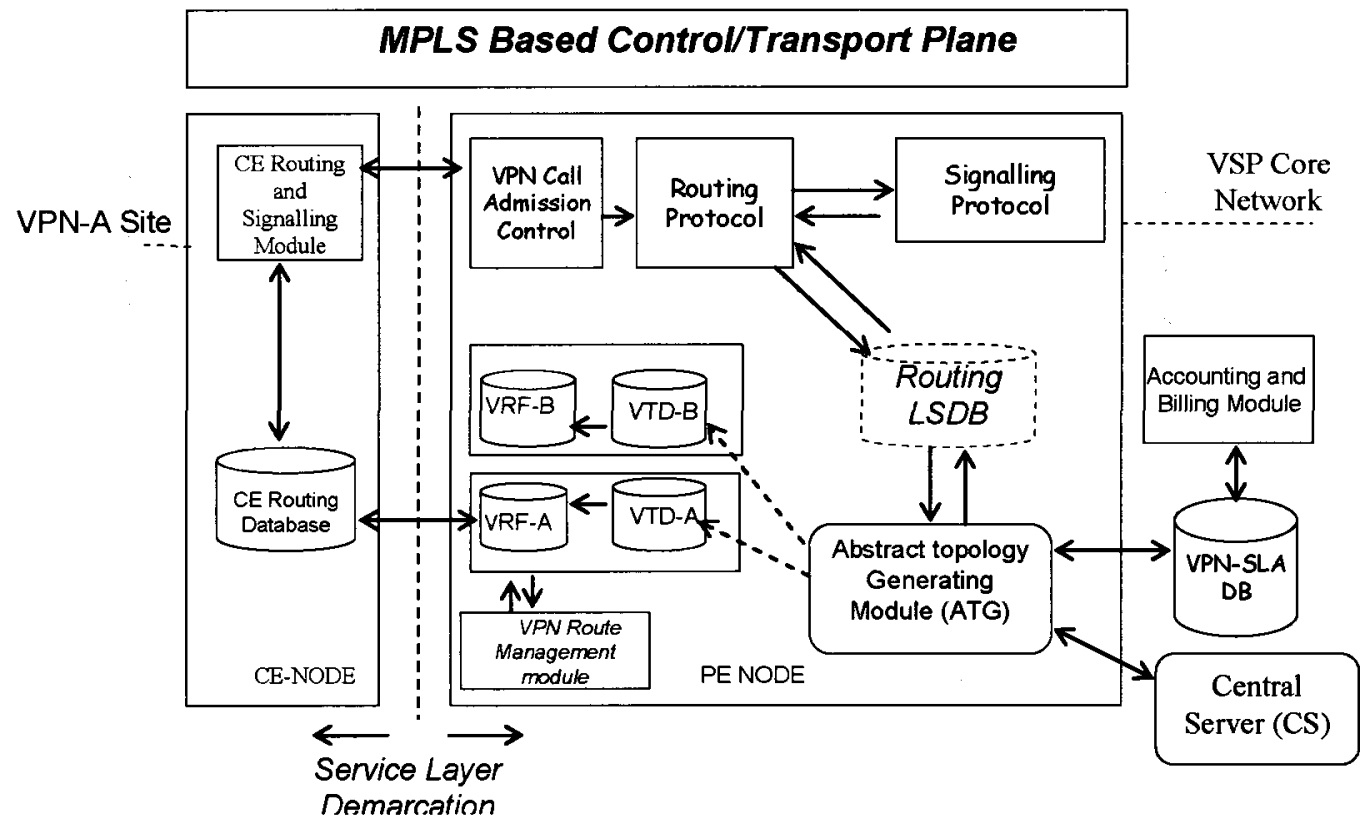

Figure 3-2: Managed dynamic VPN framework 
The proposal in [RFC4364] uses VPN specific virtual routing and forwarding (VRF) instance tables within PE routers to logically separate VPN contexts, both from a control and transport plane perspective. The additional software modules required to realize the MDVF are part of the control plane logic are shown as colored components in Fig 3.2.

The VPN SLA database (VPN-SLADB) is a database for the SLA parameters (discussed in Section 3.6) of all the VPNs subscribing to the TA service. This database could be a stand alone one or realized as an extension to VSP's customer management database that defines other services. This may be implemented as a centralized database that will be accessible to all the PE nodes or as a distributed database local to a PE node managed by the operator. This SLA database could be further used to communicate with the appropriate accounting and billing modules in order to charge for the resources held by a VPN during the usage of the TA service.

Another component which is called the abstract topology generation module (ATG), is the most important module in the context of the TA service that executes the core capacity sharing algorithms locally in the PE nodes with or without the assistance of the central server (CS). A central server is a centralized module, and its involvement depends on if the abstractions are generated in a distributed or in a centralized manner; the differences between the two modes of TA generation are discussed in the next chapter. The ATG module makes decisions on how to share and expose the resources of the core network among the VPNs, while taking into consideration the SLA parameters negotiated between the VPN and the VSP. One key VSP policy is related to fairness; this policy decides the criteria for logically partitioning the core resources from which the 
TAs are generated. The notion of fairness, as applicable to TA service, will be elaborated on in the next chapter (Section 4.2). The ATG module in the PE nodes also maintains timer based interrupts during which the abstract topologies are refreshed regularly and updated to the VPN specific VPN abstract topology database (VTD). The VTD has a VPN scope, and as a result, it can be considered an extension of the VRF, which in the current solution [RFC4364] is the database of VPN site-to-site routing information or can be implemented as a standalone database. The updates to abstract topologies from the above discussed ATG module are stored in this database.

The updates to the VPNs are tied to the abstract topology refresh interval metric, a parameter negotiated between the VPNs and the VSP (discussed in Section 3.6). These updates to the VPN CE node can be sent over as part of the LSA updates of a protocol, such as OSPF. [RFC4577] proposes the use of OSPF as the peering protocol between the CE and PE node for BGP/MPLS based IP-VPN solution [RFC4364] to exchange reachability information. This recommendation to use OSPF can also be appropriately extended to flood the abstract topology information as link state updates as part of TA service to the VPNs. These abstract topology information, once populated in the client routers, can be used by the VPNs to compute end-to-end path traversing the VSP's core network and check on the availability of the desired QoS.

\subsubsection{Use of Topology Abstraction for CE Router}

With reference to the time-line representation of the interaction between $\mathrm{CE}$ and the PE nodes in Fig. 3.3, we discuss how a request for a path capacity, which originates from a 
$\mathrm{CE}$ router $(\mathrm{S}-\mathrm{CE})$ to a destination $\mathrm{CE}$ router $(\mathrm{D}-\mathrm{CE})$ of the same VPN, is handled using the TA information provided to the CE routers. The TA provided to the S-CE by the PE node (S-PE) serving the VPN site is installed in its routing database in the same way as any other link state update from its peer routers. To enable specialized treatment to the abstract topology information from the other routing information local to the VPN site the $\mathrm{CE}$ routers are assumed to have the ability to distinguish between the normal link state information and the virtual link information associated with the abstract topology in its routing database. The TA is used by the S-CE node locally in order to compute paths to remote D-CE nodes belonging to the same VPN.

When there is a demand to provision a path with certain bandwidth requirement, the S-CE node applies a suitable algorithm (such as the constrained Dijkstra's algorithm [Ahu93] which is widely used) in order to test the feasibility of the path (i.e., if a path with the requested bandwidth is likely to be present in the core network). Upon passing the feasibility test, the path request is forwarded to the S-PE node that first determines the destination PE node (D-PE) hosting the VPN site where D-CE node is located. Once this is done, the S-PE tests for the availability of a path to the D-PE in the core and also checks the access link capacity connecting D-PE and D-CE for availability of sufficient bandwidth so as to meet the QoS requirement.

Based on the outcome of the path computation and successful signalling of the path to the D-PE node as well as to the neighbouring D-CE node, the S-PE node sends a positive or negative acknowledgement to the S-CE node. The PE router then reacts to the changes in the link state bandwidth updates and triggers the ATG module (Fig. 3.2) to re- 
compute the abstract topologies. The newly computed abstractions are then updated in the respective VTD for flooding to the VPNs depending on the VPN SLA parameters.

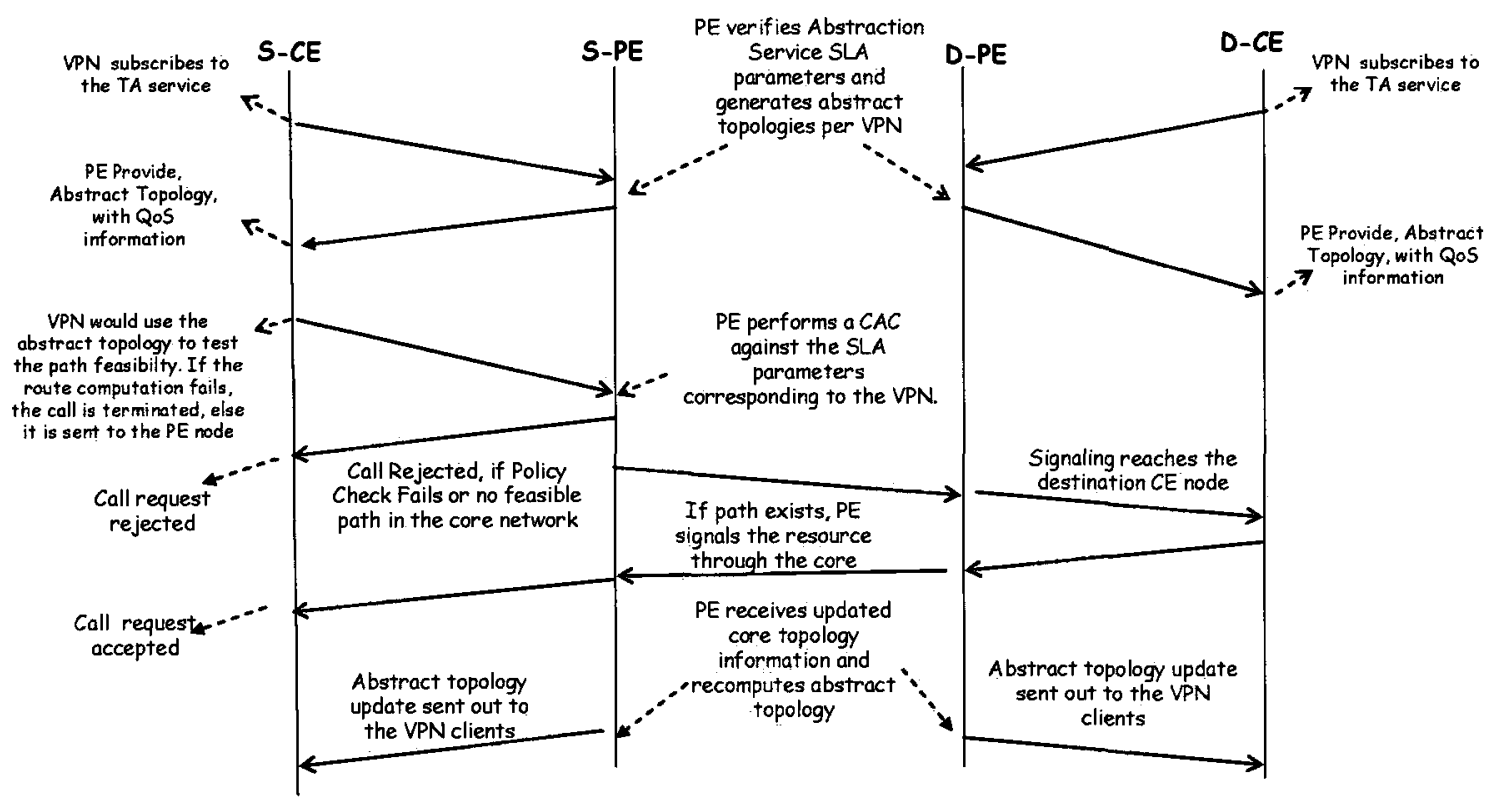

Figure 3-3: VPN topology abstraction service call request flow

\subsection{Graph Theory Notations}

Before continuing on, we introduce the graph theory notations used in the context of the TA service. Referring to Fig. 3.4, the VSP's core topology is represented as a graph $G(V, E)$. Each directed link of the network $e_{i, j} \in E$ has a capacity represented as $t_{i, j}$ (which we also denote as $t(e))$. This is the total capacity of the link. On the other hand, the capacity available on a link $e$ at any given time is called residual capacity, denoted as $r_{i, j}$ (or $r(e)$ ). $B$ represents the set of all border PE nodes in graph $G(V, E) . U$ represents the set of all VPN customers subscribing to the TA service. Each border node $b \in B$ may support multiple VPN instances identified as $U_{b}$. For a VPN instance $k \in U$, we represent the sets 
of corresponding $\mathrm{CE}$ and $\mathrm{PE}$ nodes as $C_{k}$ and $P_{k_{k}}$, respectively. The set of $\mathrm{CE}$ nodes corresponding to a VPN instance $k \in U$ hosted on a border node $b$ is represented as $C_{k, b}$.

As part of the TA service, each VPN is served with an abstract topology of type $l$. We discuss different types of abstract topologies in Section 3.7. For a given VPN $k \in U$, we represent the abstract graph as $G_{k, l}\left(V_{k}, E_{k}\right) . V_{k}$ includes the subset of PE nodes $P_{k}$, the set of CE nodes $C_{k}$, and the set of virtual nodes $Y_{k}$. Each of the nodes in the set $P_{k}$ and $C_{k}$ map to a border $P E$ and a $C E$ node, whereas $Y_{k}$ are logical node(s) virtualizing VSP's core which does not map explicitly to any of the core or border nodes. There are two types of links comprise the set of edges $E_{k}$ in the abstract topology. The first is the access link, which connects a $C_{k}$ node to a $P_{k}$ node, and the other is the set of virtual links connecting a $P_{k}$ node to a virtual node in $Y_{k}$ or another $P_{k}$ node. Broadly speaking, the virtual link $e \in E_{k}$ can be associated with a vector of abstracted QoS metrics. Here, we restrict ourselves with one abstracted metric, namely, bandwidth. Hence, for a VPN $k$ provided, with an abstract topology of type $l$, we denote the abstracted capacity associated with the virtual link connecting nodes $x$ and $y$ as $w_{k, l}(x, y)$. This bandwidth represents the capacity exposed by the VSP between the pair of nodes $(x, y) \in V_{k}$. The remote access link connectivity information flooded as part of the abstraction will carry the available capacity of the physical link corresponding to that remote access link. A VPN $k$ is also associated with a set of SLA parameters, and this is represented as $T S_{k}$; the contents of the SLA will be discussed in Section 3.6. The remaining notations would be introduced as and when required in the subsequent discussions. 


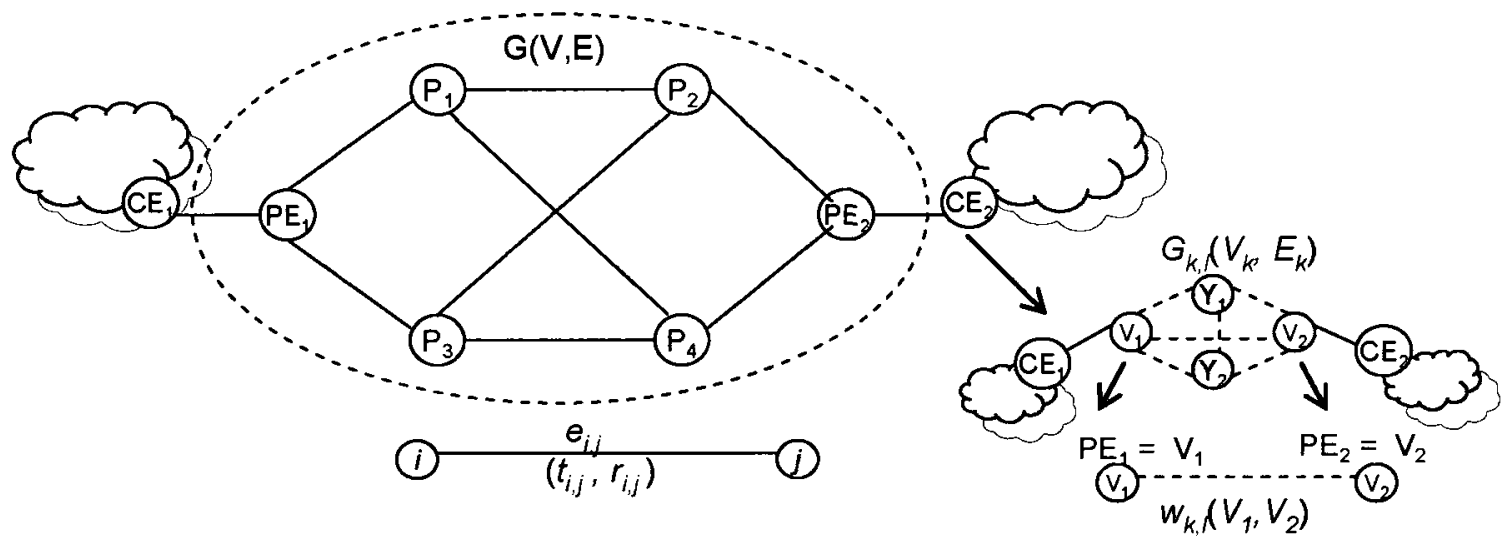

Figure 3-4: Graph theory notations for TA service

\subsection{TA Service SLA Definition and Parameters}

To enable the VSP to use TA service to generate service differentiation among the VPN customers, we propose a new set of topology abstraction SLA (TA-SLA) parameters, which allows the VSP to customize the properties of the TA service to the requirements of the VPN. In addition to service differentiation, need for separate SLAs for different VPNs is motivated by factors such as criticality of the applications for which TA service will be used and the fact that each VPN would have different requirements, in terms of both correctness of link state information and performance expected from such a service. We next discuss the elements of the TA-SLA.

Abstraction Topology Type Parameter: This parameter represents the type of abstract topology generated by the VSP for a VPN. The VSP uses this parameter to generate an abstract graph with a certain granularity before sharing it with the VPN. Note that an abstract graph with more granularity in terms of connectivity and correctness of link state 
information is expected to enable a VPN to request bandwidth more intelligently, which is expected to improve its call performance in a dynamic call request scenario. The optimizing objective for any form of TA is to minimize the complexity with respect to the granularity of the abstraction, while at the same time, maximizing the accuracy of the topology metric information that is being abstracted.

In this thesis, we have considered four forms of abstract topologies: fully meshed abstraction (FMA), source-star abstraction (SSA), star abstraction (SA), and simple node abstraction (SNA), which are also the most well studied forms of abstractions in the context of hierarchical routing literature. We discuss the properties of these TAs in the context of a VPN service later on in the section. We use the notation $T_{k}$ to denote the TA type subscribed by VPN $k$. In this case, the parameter would be one of FMA, SSA, SA, or SNA types. $G_{k, l}\left(V_{k}, E_{k}\right)$ will denote the abstract topology $l$ corresponding to VPN $k$, where the $l$ represents one of the four abstract topologies FMA, SSA, SA, or SNA.

Abstract Topology Link Metric: This parameter identifies the choice of QoS metric information that is required to be associated with the virtual links of the abstract topology chosen as part of the abstraction topology type parameter. It is not necessary that all the VPNs subscribe to the same set of QoS metric information. The choice of QoS metric associated with the abstract topology could be determined by the purpose for which the TA service will be used for. The problem with abstracting link metric information to IPVPNs can be classified into two categories: single metric abstraction and multiple metrics abstraction; a survey of literature on the topic of aggregating single and multiple link 
metrics in the context of hierarchical routing was presented in Section 2.7, it is also applicable to this problem of aggregating information to IP-VPNs. In this thesis, we deal only with the abstraction of the available capacity associated with the links of the core VSP network. The virtual link metric associated with the nodes $x$ and $y$ for a VPN $k$ of the abstracted graph $G_{k, l}\left(V_{k}, E_{k}\right)$ is represented as $w_{k, l}(x, y)$.

Abstract Topology Refresh Interval Metric: The importance of this parameter is linked to the fact that the VSP provides periodic updates about resource availability by flooding the TA periodically in order to synchronize the core state information with that of the VPN's view of the core network. From this, we can see intuitively that the choice of this parameter decides the correctness of the TA information at any given point of time in relation to the link state of the VSP's core network. In a dynamic call request scenario, the ideal refresh rate (inverse of the refresh interval) desired by a VPN is expected to be a function of the mean arrival rate of bandwidth requests, dynamic nature of the state information in the core network, which will depend on factors like the load offered by all the VPNs and time of day, and the tolerance for the control overhead by the CE routers due to the frequency of update of abstraction information from the PE nodes. The abstract topology refresh rate negotiated by the VPN is also influenced by both the abstraction topology type and the abstract topology link metric SLA parameters chosen by the VPN. For instance, a VPN subscribing to a low granular abstract topology type would require lower refresh rates in comparison to a VPN with a higher granular abstraction, because of the existence of higher number of virtual links in a higher granular abstraction. Also, a 
highly dynamic abstract topology link metric, such as available bandwidth, would require frequent updates compared to static parameters like hop counts. The choice of abstract topology refresh interval metric for a VPN $k$ is represented as $R_{k}$.

In addition to the TA-SLA parameters mentioned above, a full realization of the TA service in practical networks would involve many other service parameters, such as: aggregate bandwidth that can be requested by the VPN in an hour/day/month; service charging model; service downtime guarantees; the protocols applied between the PE and the $\mathrm{CE}$ device for providing the abstract topology updates; and the signalling mechanism used to request bandwidth.

\subsection{Topology Abstraction Types}

We next discuss the properties of the four abstraction types that have been considered in this research as part of the TA service to the IP-VPNs: fully meshed abstraction, sourcestar abstraction, star abstraction, and simple node abstraction. We elaborate on the different TA types, referring to Fig. 3.5, which shows a VPN service provider (VSP) providing TA service to two different VPN clients. We also discuss how each of the abstractions can be derived from the fully meshed type of abstraction. The need for generating the abstractions from a fully meshed abstraction is to enforce fairness which will be elaborated in the next chapter (Section 4.2). Figs. 3.6-3.9 show the abstractions that are being considered. The two headed arrow in the figure represents a pair of oppositely directed links. The straight arrow in the figures represents a physical link, while the dashed one represents a virtual link. In the next chapter, we propose a 


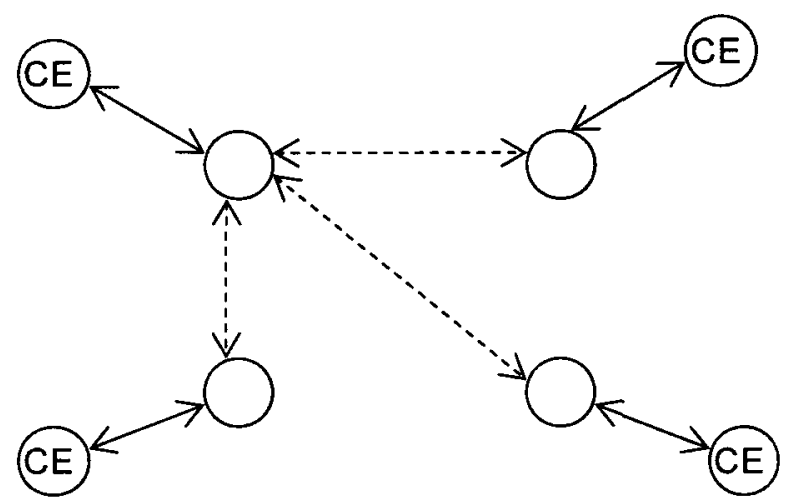

Figure 3-7: Source-star abstraction

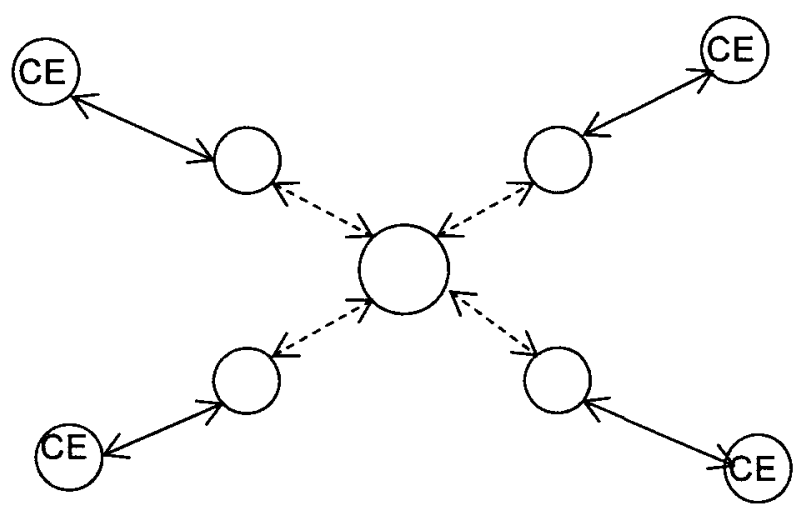

Figure 3-8: Star abstraction

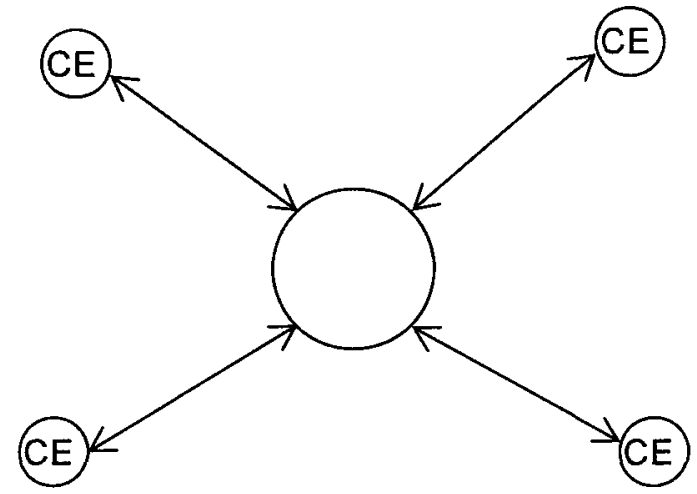

Figure 3-9: Simple node abstraction 
methodology to calculate the bandwidth for each virtual link in the fully meshed abstraction; from this, as we discuss next, one can obtain the bandwidth for the virtual links of the other forms of abstract topologies.

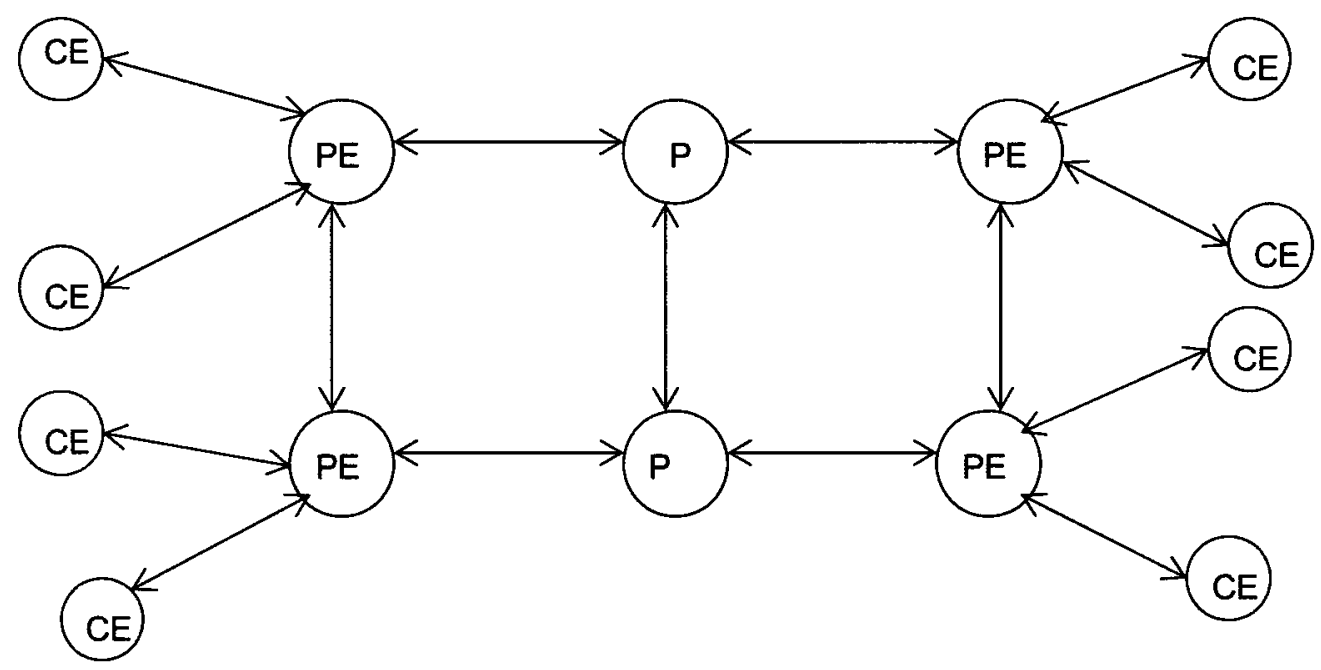

Figure 3-5: VSP providing topology abstraction service

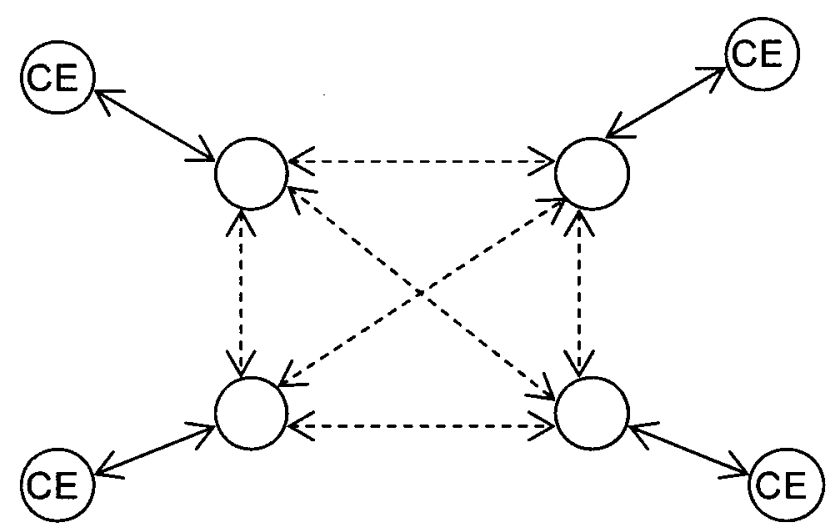

Figure 3-6: Fully meshed abstraction 
Fully Meshed Abstraction Topology: This abstraction is shown in Fig. 3.6. Here, for a VPN $k \in U$ the set of CE nodes $C_{k}$ is provided a virtual topology that is a full mesh of virtual links between all the PE nodes in $P_{k}$. A drawback with this abstraction is the $O\left(\left|P_{k}\right|^{2}\right)$ virtual link updates that would have to be flooded to the VPN client nodes periodically, whose interval is determined by the TA-SLA parameter $R_{k}$. The total message complexity of updating this form of abstraction is $O\left(\left|P_{k}\right|^{2}+\left|C_{k}\right|\right) . O\left(\left|C_{k}\right|\right)$, which accounts for the overhead of updating the VPN remote access link (connecting the CE and the PE nodes) is the common complexity component for remaining cases as well. The fully meshed abstraction provides the least distortion of the abstracted capacity information that is provided to the VPNs. Another important property of this abstraction is that it provides choice of multiple virtual paths between two virtual nodes.

Source-Star Abstraction Topology: This type of abstraction, illustrated in Fig. 3.7, has been adopted from [Kor00]. In this case, a VPN is provided an abstraction that is a source-rooted tree. Here, the root of the tree is the PE node computing abstraction for the corresponding VPN. The other nodes of the source-star topology are the PE nodes $P_{k}$ on which VPN of the same type are hosted. This form of abstraction is based on the fact that redundant virtual paths from a fully meshed scenario are not useful for path computation except when a CE node is connected to multiple PE nodes. In the case where a CE node has adjacency with more than one PE node, multiple source-star abstractions would have to be provided to the $\mathrm{CE}$ node in order to take advantage of the routing diversity due to multiple PE connectivity. This scheme scales better than the fully meshed scheme due to 
its linear overhead complexity. In this case, for a case of single CE and PE connectivity and for a given VPN $k$, the total message complexity of updating a CE is $O\left(\left|P_{k}\right|+\left|C_{k}\right|\right)$. The form of abstraction can be derived from the fully meshed abstraction for a given VPN $k$ by pruning the virtual links from the set of links connecting the nodes in the set $P_{k}$ that are not connected to the root PE node $b$ which computes the TA for the set of CE nodes $C_{k, b}$ of the VPN.

Star Abstraction Topology: This abstraction is shown in Fig. 3.8. In this scheme, the abstract topology contains all the border nodes hosting the VPN, i.e. the set of nodes $P_{k}$, and virtualizes the remaining network as a virtual node $v$. In this case the virtual link edges of the abstract topology correspond to the connectivity between the border nodes and the virtual node. For a given instance of VPN $k$, the spokes of the star topology connects a node $u \in P_{k}$ to the virtual node $v$ bi-directionally. The bandwidth of a virtual link can be assigned in multiple ways; one way is to assign to each virtual link connecting a node $u \in P_{k}$ to the spoke node $v$ the bandwidth that is the average of the bandwidths of all the virtual links $(u, w)$ in the fully meshed abstraction with $w \in P_{k}$ and $w \neq u$; the equations to compute these virtual link capacities are given in (3.1) \& (3.2). In these equations, $w_{k, S A}(u, v)$ represents the capacity associated with the directional virtual link $(u, v) \in E_{k}$ corresponding to abstract graph $G_{k, S A}\left(V_{k}, E_{k}\right)$. Summations are over all nodes in $P_{k}$. Another approach, but an aggressive one, to compute the virtual link capacity is to set this to the maximum of the capacities of the virtual links in the fully meshed 
representation. For any given VPN $k$, the total message complexity to flood a star abtract topology is $O\left(\left|P_{k}\right|+\left|C_{k}\right|\right)$.

$$
\begin{array}{ll}
w_{k, \mathrm{SA}}(u, v)=\sum_{w} \frac{\left(w_{k, \mathrm{FMA}}(u, w)\right)}{\left(\left|P_{k}\right|-1\right)} & \forall w \in P_{k}, w \neq u \\
w_{k, \mathrm{SA}}(v, w)=\sum_{l} \frac{\left(w_{k, \mathrm{FMA}}(l, w)\right)}{\left(\left|P_{k}\right|-1\right)} & \forall l \in P_{k}, l \neq w
\end{array}
$$

Simple Node Abstraction Topology: This is the least granular of all the abstraction services. Here, the core network is completely virtualized as a single node, as shown in Fig. 3.9. The VPN CE nodes $C_{k}$ in this case is provided only the update of the available bandwidth on the remote VPN access links. The complexity of updating a VPN with this form of abstraction is $\mathrm{O}\left(\left|C_{k}\right|\right)$. This abstraction is derived from the fully meshed abstraction for VPN $k$ by pruning all the virtual links connecting the set of nodes $P_{k}$ and virtualizing the set of nodes $P_{k}$ as a single virtual node.

\subsection{Summary}

In this chapter, we introduced the need for topology abstraction service to IP-VPNs; the objectives we intend to achieve with this service; and an approach to realize it in the context of a VSP's environment using the managed dynamic VPN framework. We also discussed the need for dynamic VPN service (DVS) for IP-VPNs and how TA service can be used to enable it along with the key differences with respect to its usage in hierarchical routing. Considering the objectives of the TA service, we proposed a novel SLA definition called TA-SLA that is required to realize TA service in a practical context. We proposed the realization of TA service in the context of the MDVF through 
various control and service plane extensions to well known BGP/MPLS based IP-VPN solution. We ended our discussion by introducing the different TA types that have been considered in this thesis and how they can be generated from a fully meshed topology abstraction. 


\section{Chapter 4 \\ Topology Abstraction Generation and Evaluation}

\subsection{Introduction}

In Section 4.2 of this chapter, we present the sequence of steps for generating abstract topologies customized to each VPN depending on its negotiated TA-SLA parameters and satisfying the fairness requirements of the VSP's TA service. In Section 4.3, we evaluate the performance of the TA service in the context of the MDVF for various TA types using simulation analysis. Our evaluation is with respect to VPN call performance, network utilization, and fairness objectives. We also study how the TA-SLA parameters can be used to generate service differentiation in the context of MDVF.

\subsection{Topology Abstraction Process}

One of the significant benefits of abstracting VSP's core network and QoS information is to improve the call performance of the VPNs that require dynamic bandwidth service, while minimizing the overhead for sharing information with the VPNs. The performance 
of the TA service depends significantly on the schemes used to generate the abstract topologies. The abstract topologies for the VPNs may be computed either in a decentralized or in a centralized manner. We discuss these two modes of generating VPN TAs next.

In the case of decentralized mode of TA generation, the process of generating an abstract topology is very similar to that applied in the context of hierarchical routing, such as in PNNI. With this mode of TA generation, the PE nodes hosting a set of VPNs generate abstractions independent of other PE nodes in the core network. This mode of TA generation minimizes the control logic overhead; since the PE nodes only use the local routing topology database and TA service specific information to generate the TAs for each VPN. A drawback of this TA generation process is the oversubscription issue, which results from exposing more than the available core capacity to the VPNs. Although this approach will help improve statistical multiplexing of VPN bandwidth requests and core network utilization, this may also result in call rejections resulting in poor VPN call performance in the context of TA service particularly at high load conditions.

In a centralized approach, the problem of determining the TA for each VPN is resolved centrally. In this case, a central server (CS) (Fig. 3.2) generates the VPN TAs on behalf of all the PE nodes and shares this information with the border nodes, which then share this information with the CE nodes. The key advantage of this process of TA generation is that it mitigates the problem of oversubscription encountered in the decentralized mode of TA generation. Despite the fact that this TA generation process is 
expected to deliver better VPN call performance, this approach may also turn out to be conservative in terms of network utilization under dynamic VPN call arrival scenarios.

Both the decentralized and centralized approaches assume the availability of core network topology, either locally in a border node for decentralized implementation or in a centralized module such as the CS for centralized abstraction. In addition, the algorithms also require VPN specific information, which would include information about the VPNs hosted on each border node, along with the residual capacity of the VPN access link connecting the PE and the $\mathrm{CE}$ nodes. The core topology information is readily available as part of link state protocols, such as OSPF and ISIS, which are the protocols of choice in most VSP networks. The VPN specific information required by the abstraction schemes can be obtained from the MDVF service components, specifically the VPNSLADB module, introduced in Section 3.4.

Next, we discuss the steps followed to generate TAs in a decentralized context. This process of TA generation is also applicable for the centralized mode, but with some differences which we elaborate in Chapter 6. Before going into its details, we first discuss the fairness policy that is applied to generate TA's in a fair manner applicable to both modes of TA generation.

\subsubsection{VPN TA Generation Policy for Fairness}

The abstract topology provided to a VPN as part of the TA service is a summary of the VSP's core topology, which is derived from an intermediate subgraph that is generated for each VPN called as the partition subgraph. The abstract topology is then derived from 
this partition subgraph. Hence, the fairness policy in the context of the TA service has implications at two stages of the TA generation process: first, when partition subgraphs are computed for the VPNs, which involves determining the edges of the core graph and the logical residual link capacity to be allocated for the VPNs as part of its partition subgraphs; second, when these graph partitions are used to generate abstract topologies for the VPNs based on the negotiated TA-SLA parameters. The faimess decision in this step is the capacity associated with the virtual links of the abstract graph corresponding to each VPN. The abstract graph is the graph that is actually advertised to the VPN.

While computing the partition subgraphs during the subgraph computation phase (discussed in Section 4.2.2), the VSP could adopt a policy to either treat all the VPNs equally; or to partition the link resources among the VPNs based on their SLA parameters such as customer priority or TA-SLA parameters. For our study, during the subgraph computation phase we treat all the VPNs equal. For the decentralized mode of TA generation, the VPN subgraphs are generated independent of one another assuming the availability of the total residual link capacity; for the centralized mode of TA generation, wherein the partition subgraphs are computed at the same time for all the VPNs, we assume that the link's residual capacity is shared equally among all the concerned VPNs. It should be noted that our approach discussed in this thesis can also be generalized to the policy where bandwidth sharing is based on TA-SLA parameters rather than equal priority of the residual bandwidth of each link to all the VPNs.

This fairness policy associated with the subgraph partition computation is applied as part of the TA generating algorithm used to compute the partition subgraphs. (These 
schemes are discussed in detail in Chapters 5-6 where we propose several decentralized and centralized abstraction schemes.)

In the second stage of fairness decision, the VSP has to decide how the resource computed from the partition subgraphs be exposed to the VPNs as abstracted topology. An important factor to consider here is the TA-SLA parameters, particularly the abstraction topology type parameter introduced to enable service differentiation based on the topology granularity and the correctness of link metric information associated with abstract graph. To enable this service differentiation, in this thesis, we assume that the VSP adopts a model where the goal is to always expose the available capacity equally among all the VPNs that have subscribed to the same abstract topology type parameter $T_{k}$. This fairness policy is applied in the same way for both the mode of TA generation. This fairness policy is applied locally by each border node $b$, while generating abstract topologies for the set of VPNs $U_{b}$ hosted by it. The criterion we adopt can be stated as follows:

For a pair of VPNs $\left(i_{2} j\right) \in U_{b}$ with the same abstract topology type parameter $l$, with abstract graphs $G_{i, l}\left(V_{i}, E_{i}\right)$ and $G_{j, l}\left(V_{j}, E_{j}\right)$ and with a common pair of nodes $x$ and $y$, the VSP imposes the rule that the exposed capacity associated with virtual link connecting the nodes $x$ and $y$ should be equal, that is $w_{i, l}(x, y)=w_{j, l}(x, y)$. This guarantees that two VPNs with the same TA type will be exposed the same amount of bandwidth for each virtual link in the abstract topology. 
To realize the above two fairness policies, we propose a three-stage approach for generating TAs for the VPNs. The purpose of the first stage is to compute the partition subgraphs and generate fully meshed abstractions from these subgraphs for all VPNs.

The second stage applies the fairness criterion to all the fully meshed abstractions. The third stage generates different abstractions by converting the fully meshed TA for each VPN to the requested TA by using the method discussed in Section 3.7.

As can be seen easily, the first two stages make VPNs with different topologies comparable by generating all VPNs with the same topology, i.e. the fully meshed topology. Different topologies are then derived by converting the common fully meshed topology to one required by the abstract topology type TA-SLA parameter. The reason we choose fully meshed topology as the starting point to impose the second fairness policy is due to the fact that it carries the most information about the available core resources; converting fully meshed topology into requested topology is an information reduction process, which naturally leads to service differentiation. Therefore, our novel three-stage TA generation approach can provide both fairness and service differentiation.

\subsubsection{Steps for Topology Abstraction}

Fig. 4.1 shows the steps involved in the TA abstraction process executed by a PE node $b$ in order to generate abstractions for the VPNs hosted by it, represented as $U_{b}$. The border node $b$ is assumed to have access to the routing link state database, VRF configuration, and TA-SLA information required for TA generation. The procedure basically involves 
iterating over the set of VPNs it hosts for which abstract topologies needs to be generated.

In Fig. 4.1, Step 1 identifies the subset of border nodes $P_{k}$ and $C_{k, b}$ for each VPN $k \in U_{b}$. Note that $P_{k}$ is the subset of border nodes that hosts VPN $k$, and $C_{k, b}$ is the subset of CE VPN nodes of VPN $k$ served by border node $b$ to which the final TA information has to be flooded to.

In Step 2, we generate the partition subgraphs. This is also the step where the first fairness criteria discussed in the previous section is applied. Generating a partition subgraph basically involves deriving a subgraph of the VSP's core graph by the border node for each VPN from which the fully meshed abstract topology is derived. As the goal is to generate a fully meshed abstraction in Step 3, for the decentralized mode of TA generation multiple partition graphs will have to be computed with respect to all the nodes $P_{k}$ corresponding to the VPN $k \in U_{b}$.

Step 3 uses the computed partition graphs $S\left(V_{k}, E_{k}\right)$ generated in the previous step to derive a fully meshed abstract topology for the VPN $k$, which we represent as $G_{k, F M A}\left(V_{k} E_{k}\right)$. This step entails assigning the virtual link capacity of an edge $(x, y) \in E_{k}$ equal to the bottleneck capacity of the path connecting nodes $x$ and $y$ in graph $S\left(V_{k}, E_{k}\right)$.

In Stage 2, Steps 4-6 apply the second fairness criteria discussed in the previous section. This stage inputs the fully meshed abstractions computed for all the VPNs in the previous stage. Steps 4-6 apply the fairness criteria as follows: for each type of abstract topology type parameter $l \in\{F M A, S S A, S A, S N A\}$ supported by the VSP, we iterate over all pairs of border nodes in the set $B_{b}$, where $B_{b} \subset B$ is the set of all border nodes that has at 
least one VPN common to the set of VPNs, $U_{b}$, hosted on border node $b$ i.e. $B_{b}=\left\{\cup_{k \in U_{b}} P_{k}\right\}$. For each pair of border nodes $\left(b_{1}, b_{2}\right) \in B_{b}$, we determine the set of VPNs sharing the pair of border nodes and subscribing to the same abstract topology type parameter $l$. We represent this set as $Z_{l}\left(b_{1}, b_{2}\right)$. For this set of VPNs in $Z_{l}\left(b_{l}, b_{2}\right)$, for all $z \in Z_{l}\left(b_{1}, b_{2}\right)$ we set the virtual link capacity $w_{z, F M A}\left(b_{1}, b_{2}\right)$ in the abstract graph $G_{z, F M A}\left(V_{z}, E_{z}\right)$ to the minimum of virtual link capacities in the fully meshed abstractions (previously computed in Steps 2-3) corresponding to the set of VPNs in $Z_{l}\left(b_{l}, b_{2}\right)$. For the case where there are no VPNs sharing a particular combination of border node pair, the set $Z_{l}\left(b_{1}, b_{2}\right)$ will be null.

In Stage 3, iteration is carried over the set of VPNs $U_{b}$. Step 7 applies the topology type SLA parameter $l \equiv T_{k}$ to the fully meshed abstract graphs $G_{k, F M A}\left(V_{k}, E_{k}\right)$ so as to derive the desired topology abstraction $G_{k, l}\left(V_{k}, E_{k}\right)$, which was discussed in Section 3.7. In Step 8, the PE updates the set of $\mathrm{CE}$ nodes $C_{k, b}$ with this abstract topology with a periodicity determined by $R_{k}$.

\section{Abstract Topology Generation Process}

Input: $G(V, E), U_{b}, T S_{k} \forall k \in U_{b}$.

Output: $G_{k, l}\left(V_{k}, E_{k}\right), \forall k \in U_{b}$.

begin

/Stage 1: Generate partition subgraphs and fully meshed abstraction begin

for each VPN $k \in U_{b}$,

Step 1 : find set $P_{k} \subset B$ and $C_{k, b} \subset C_{k}$ for VPN $k$; 
Step 2 : for VPN $k$ and for nodes in $P_{k}$ compute partition subgraphs $S\left(V_{k}, E_{k}\right)$;

Step 3 : generate fully meshed abstraction graph $G_{k, F M A}\left(V_{k}, E_{k}\right)$ from $S\left(V_{k}, E_{k}\right)$; end;

/Stage 2: Apply fairness criteria to the set of VPNs $U_{b}$ begin

determine set $B_{b}$;

for each abstract topology type parameter $l \in\{F M A, S S A, S A, S N A\}$,

Step 4: for each pair $\left(b_{1}, b_{2}\right) \in B_{b}$,

Step 5: determine the set $Z_{l}\left(b_{1}, b_{2}\right)$, calculate $b w=\min _{z \in Z_{l}\left(b_{1}, b_{2}\right)}\left\{w_{z, F M A}\left(b_{1}, b_{2}\right)\right\}$; for each VPN $x \in Z_{l}\left(b_{1}, b_{2}\right)$,

Step 6: $\quad$ set $w_{x, F M A}\left(b_{1}, b_{2}\right)=b w$;

end;

/Stage 3: Generate the required abstraction applying $T_{k}$

begin

for each VPN $k \in U_{b}$,

Step 7: apply $l \equiv T_{k}$ to generate $G_{k, l}\left(V_{k}, E_{k}\right)$ from $G_{k, F M A}\left(V_{k}, E_{k}\right)$;

Step 8: update $C_{k, b}$ of VPN $k$ with $G_{k, l}\left(V_{k}, E_{k}\right)$ with a periodicity of $R_{k}$;

end;

end;

\section{Figure 4-1: Topology abstraction process for TA Service}

We next present simulations results that study the feasibility and usefulness of TA as a service for IP-VPNs in the context of MDVF, and more importantly, evaluate how the TA-SLA parameters can be used to generate service differentiation. For the simulation analysis, we use the maximum capacity scheme for Step 2 and Step 3 to compute the 
fully meshed abstraction $G_{k, F M A}\left(V_{k}, E_{k}\right)$. This scheme is elaborated in Section 5.2.1, it assigns capacity of the widest path between a node pair as the capacity of the virtual link.

\subsection{Simulation and Performance Evaluation}

In this section, we discuss the performance results of the abstract topology types (FMA, SSA, SA, SNA) discussed in Section 3.7 using the VPN TA generation process discussed in the previous section. This section will also show how service differentiation can be achieved using the TA-SLA parameters, i.e. the abstract topology type $\left(T_{k}\right)$ and abstract topology refresh interval metric $\left(R_{k}\right)$, discussed in the previous chapter, Section 3.6. The objectives of this simulation study are as follows:

1. Establish the usefulness of the TA service as a way to share the core capacity information with the VPNs that enables them to seek capacity in an intelligent manner; assess whether topologies of various granularities can be used to provide differentiated managed VPN service; study the performance of the TA service with respect to the fairness policy discussed in Section 4.2.1 and the effect choice of $T_{k}$ has on network utilization.

2. Study the effectiveness of using TA-SLA parameter $T_{k}$ and $R_{k}$ as a service differentiation parameter.

3. To study how the TA-SLA parameter $R_{k}$ could be used in combination with $T_{k}$ in order to provide dynamic VPN service with varying service quality. 
Before we discuss the results of our simulations, we introduce the performance metrics used to compare the performance of the abstraction schemes and the simulation scenario used to evaluate the objectives.

\subsubsection{Performance Metrics}

In order to define the performance metrics associated with the TA service, we use Fig. 4.2, which shows the tree of various possible outcomes of a call originating from a VPN that is computing a path using the abstract topology provided to it by the VSP.

Starting from the root, denoted as (1), in Fig.4.2, a VPN call request could have two possible outcomes. The first outcome is when a CE node does not find a feasible path and terminates the call locally. This is identified as (3). The second outcome is that the route computation executed at the $\mathrm{CE}$ node does find a path satisfying the QoS constraints and sends the request to the corresponding PE node (2). For the case where the path could not be found, we could have two possibilities. The first possibility is that the call has been wrongly terminated due to stale abstraction information, in which case we count it as a 'Miss' call (6). The alternative possibility is that the call has been correctly terminated which correlates with the VSP's state of inability to find the required path. We label such a call as a 'Hit' call (7). For the case where a path is deemed to exist by the VPN, there can be two outcomes. The first is that the call does go through and CE receives a positive acknowledgement from the PE (4). The other is that the call crankbacks because of the inability of the PE node in the VSP to route the call (5). 
Based on the above call request possibilities, we define our performance metrics as global metrics. Hence, the related metrics are kept track of centrally for all the CE nodes of a VPN during the course of the simulation.

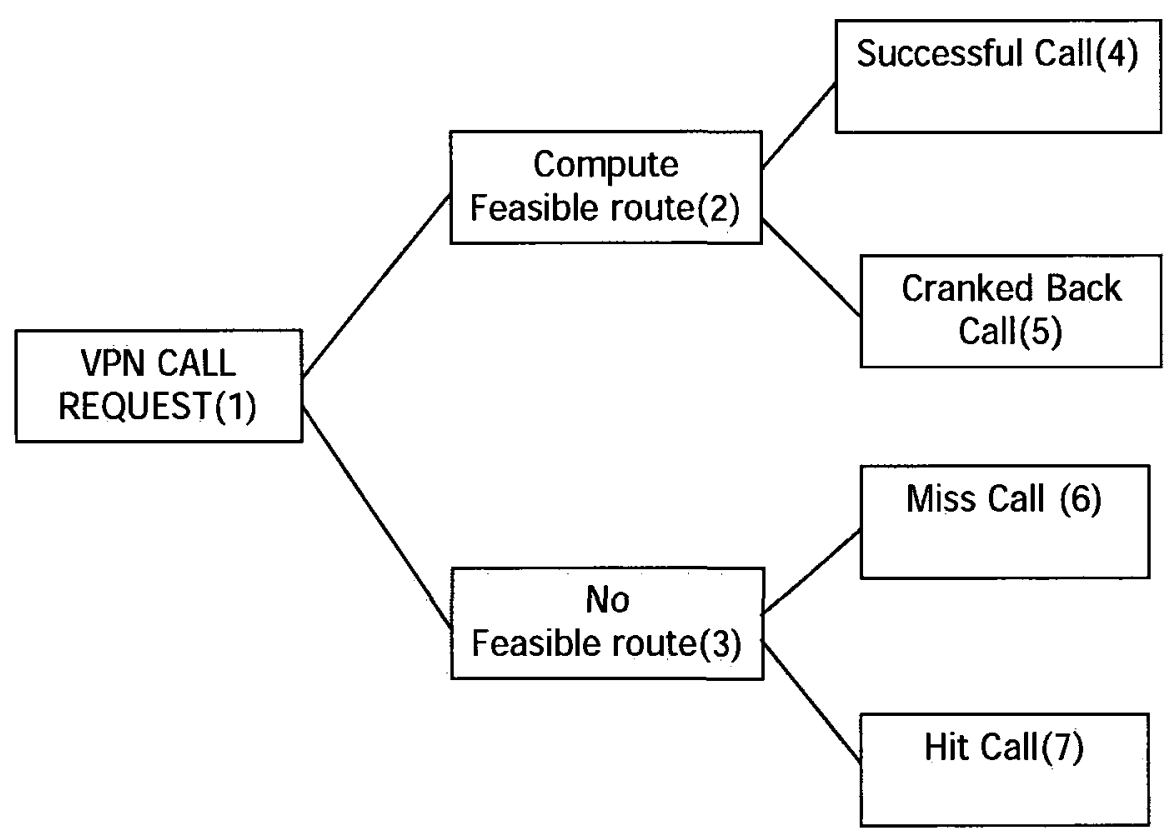

Figure 4-2: VPN call request scenarios

Success Ratio: The success ratio is a measure of a VPN making a right routing decision using the abstraction provided to it by the VSP. This includes the case of computing a feasible path, as well as the case of rejecting a path locally at the VPN's end. The correctness of a rejected path is verified by re-computing the path with the exact state of the network.

Success Ratio $=\frac{\text { Number of calls correctly accepted }+ \text { Number of calls correctly rejected }}{\text { Total number of calls }}$ 
Crankback Ratio: For the case where the client node computes a feasible path, the path would crank back, if there were no feasible paths in the core. The crankback ratio is defined as the ratio of the number of calls that have been cranked back to the total number of path requests made by the VPN.

$$
\text { CrankBack Ratio }=\frac{\text { Number of calls cranked back }}{\text { Total number of calls }}
$$

Misscall Ratio: The misscall ratio is the ratio of calls that have been wrongly terminated locally at the VPN's end (even though there is enough resource to accommodate the call) to the total number of calls originated by the VPN; ideally, a successful TA service implementation should have a miss call ratio of zero.

$$
\text { MissCall Ratio }=\frac{\text { Number of wrongly rejected calls }}{\text { Total number of calls }}
$$

Average Network Utilization: This metric refers to the ratio of total link capacity utilized by active VPN bandwidth requests (aggregate utilized link capacity) to the total link capacity (aggregate link capacity). In addition to the abstraction scheme applied to abstract the core capacity information, this factor is also influenced by the routing algorithm used to compute paths through the core network.

$$
\text { Average Network Utilization }=\frac{\text { Aggregate utilized link capacity }}{\text { Aggregate link capacity }}
$$




\subsubsection{Simulation Setup}

The simulation study is conducted using OPNET [OPNET], a well known discrete event simulator [Law00]. The topology used for studying the different scenarios is a 22 node random topology based on well-known Waxman's random graph [Wax88] model with an average node degree of $4, \alpha=0.150, \beta=2.2$; this topology is shown in Fig. 4.3. In order to validate our results over other standard topologies, we also study the performance with respect to two other topologies shown in Fig 4.4 and Fig. 4.5. Figs. 4.4-4.5 are European networks referred from [Ho07]. All the results discussed have been obtained from running the simulation for 30 independent replications to achieve $95 \%$ confidence interval for an absolute error [Law00] of less than 1\%. The number of independent replications is deduced by observing the sample variance over several independent runs, and applying the approximation given in [Law00, page. 512] .

In these topologies, five nodes were randomly chosen as the border PE nodes and the remaining nodes as the core P nodes. Each of the PE nodes was configured to handle five different VPNs, four of which subscribed to the TA service. One of the VPNs was not enabled to receive any TA service; this was done in order to compare its performance with the other VPNs and study the tradeoff of enabling TA service.

For the simulation analysis, bandwidths for the access as well as the core links were initialized to 1000 units. The bandwidth requests from the VPN client nodes were modeled as Poisson arrivals. The call holding times were assumed to be exponentially distributed. Without implementing a fully fledged flooding mechanism to maintain the link state database, the simulation implements a simple logic of having the link state 
database of all the PE nodes to be in sync with the state of the network. This is ensured by having the intra-topology update interval for route computing engine in the PE nodes set to value much less than the mean arrival rate of bandwidth requests; this value is $5 \mathrm{~s}$ for our simulation analysis (Note: The time interrupts for the events are scheduled during the simulation based on the absolute simulation clock, the units of seconds has been assumed in the simulation discussion for time related metrics to present the discussion in a practical context).

In order to generate the abstraction for the previously stated objectives $1-3$, each border node applies the maximum capacity scheme (discussed in Section 4.2) to compute the partition subgraphs in Step 2 in Fig. 4.1; this subgraph is used to generate the fully meshed abstraction in Step 3. The abstract topology computed by a border PE node is flooded to the appropriate CE node on a periodic basis whose interval is defined by the parameter $R_{k}$. In order to keep the abstract topology up to date with the network state, the refresh interval for all the VPNs is chosen to be less than the mean call inter-arrival time of the VPN requests. The CE node uses the abstract topologies to compute paths for a path request. The path request type is assumed be of uni-directional path setup type; here resources during the signaling process is commited from the requesting $\mathrm{CE}$ node to the destination $\mathrm{CE}$ node and not in the reverse direction. If the constraint path computation succeeds in finding a path to the destination $\mathrm{CE}$ node, the request is forwarded to the neighboring PE node.

Once a request reaches the PE node, the request is then forwarded to the call admission control engine; this is accomplished by performing a route computation using 
the constrained Dijkstra's algorithm [Ahu93] that checks to see if the request can be admitted into the network. If successful, the path computation is signaled using an explicit path routing construct to the destination PE node serving the egress VPN CE node. If the signaling is successful, the required resource is reserved along the path, and a positive acknowledgement is sent back to the VPN CE node; else a negative acknowledgement is sent back. In addition to the call success and crankback ratios, other call scenarios states, as shown in Fig. 4.2, will result if calls are wrongly or correctly terminated locally in a CE node.

As part of the key TA-SLA parameter initialization, for all the objectives, we assume that the VPN subscribes to different abstraction schemes, i.e fully meshed abstraction (FMA), source-star abstraction (SSA), star abstraction (SA), or simple node abstraction (SNA) service. As noted previously, one of the VPNs was purposely not provided any abstraction service. This is indicated as (NA) in our results; this kind of set up was adopted to show the improvement one might achieve, even with minimum amount of topology information. 


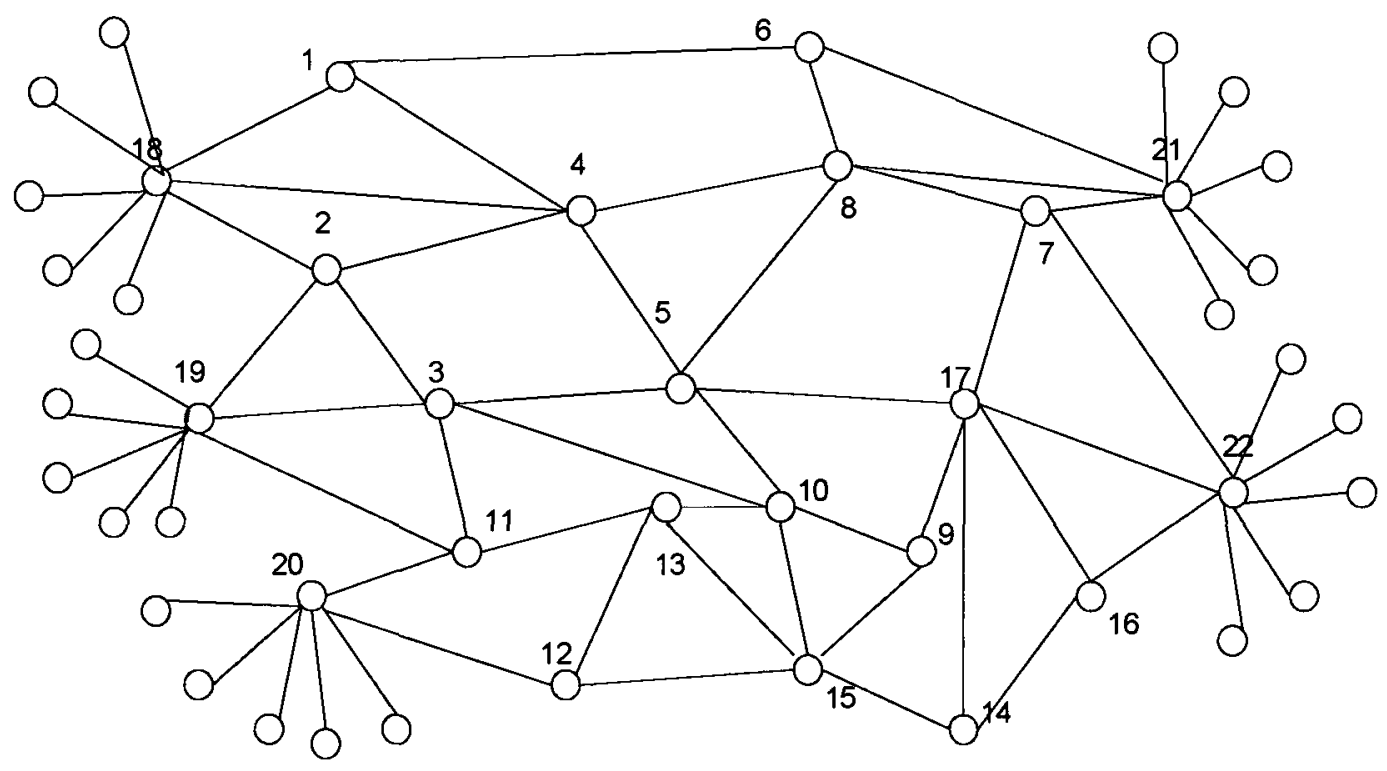

Figure 4-3: Simulation topology (Network-1)

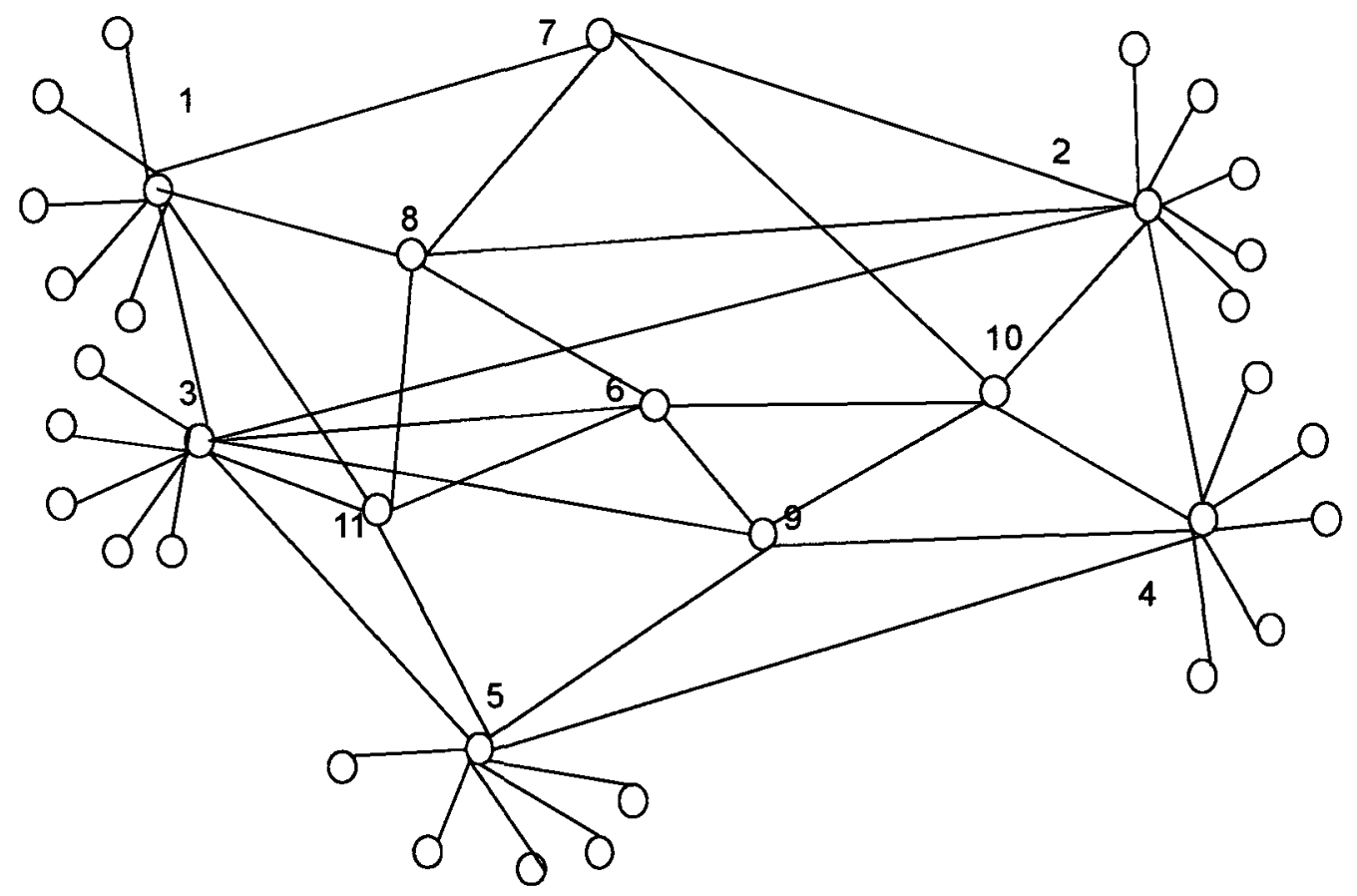

Figure 4-4: Simulation topology (Network-2) 


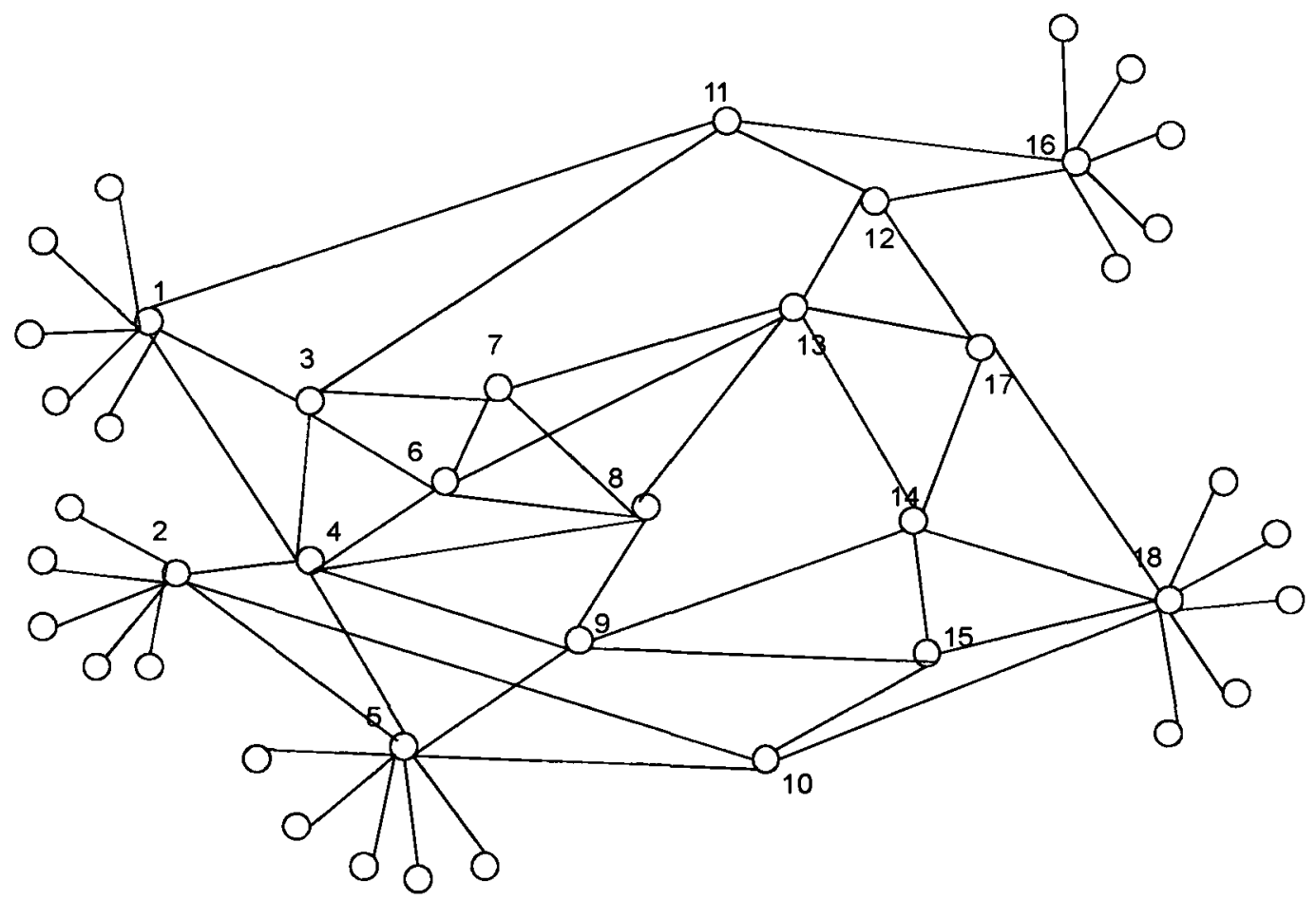

Figure 4-5: Simulation topology (Network-3)

\begin{tabular}{|l|c|c|c|}
\hline \multicolumn{1}{|c|}{ Simulation Parameters } & Obj. 1 & Obj. $\mathbf{2}$ & Obj. 3 \\
\hline VPN Mean Call Inter-arrival Time (s) (Exponential Dist.) & 100 & 100 & 100 \\
\hline VPN Mean Call Holding Time (s) (Exponential Dist.) & {$[10,1000]$} & 500 & 100 \\
\hline VPN Bandwidth Request Size (Uniform Distribution) & {$[1,500]$} & {$[1,500]$} & {$[1,500]$} \\
\hline Core Topology Update Interval (s) & 5 & 5 & 5 \\
\hline Abstract Topology Refresh Interval (VPN-A) (s) & 20 & {$[20-1000]$} & 50 \\
\hline Abstract Topology Refresh Interval (VPN-B) (s) & 20 & {$[20-1000]$} & 250 \\
\hline Abstract Topology Refresh Interval (VPN-C) (s) & 20 & {$[20-1000]$} & 750 \\
\hline Abstract Topology Refresh Interval(VPN-D) (s) & 20 & - & - \\
\hline Abstract Topology Type (VPN-A) & FMA & FMA & FMA \\
\hline Abstract Topology Type (VPN-B) & SSA & SSA & SSA \\
\hline Abstract Topology Type (VPN-C) & SA & SA & SA \\
\hline Abstract Topology Type (VPN-D) & SNA & SNA & SNA \\
\hline Abstract Topology Type (VPN-E) & NA & NA \\
\hline
\end{tabular}

Table 4.1: Simulation parameters to evaluate TA service and effectiveness of TASLA parameters 
In addition, Table 4.1 shows the important parameters used in our simulations for all the cases. We next discuss the differences in the simulation parameter settings for the different objectives with reference to Table 4.1 .

For objective 1, the VPN mean inter-arrival time was set to $100 \mathrm{~s}$ and call holding time $(H)$ was varied between $[10,1000] \mathrm{s}$, with a resulting offered load between $[0.1,10]$ Erlangs. The VPN capacity request was uniformly distributed between $[1,500]$ units. For objective 2 , the call parameters were similar to objective 1 , but since the goal here is to study the usefulness of using the $R_{k}$ metric as a service parameter, we varied the TA refresh abstraction interval in the range of $[20,1000] \mathrm{s}$. For objective 3 , the mean call parameters were the same as in the previous case, but the abstract topology refresh interval parameter $R_{k}$ was set differently for different types of abstract topology type parameter $T_{k}$, as shown in the Table 4.1 .

Next, we discuss our results for the different objective scenarios.

Objective 1: To demonstrate the usefulness of TA service and show how topology abstraction granularity effects the call performance of the VPNs

We study this objective by studying performance of the VPNs subscribing to different abstract topology types for different load conditions. In this scenario, VPNs A-D were provided with FMA, SSA, SA, and SNA abstractions, respectively, and VPN-E was not provided any form of abstraction, which we indicate as NA in our results. M 
Figs. 4.6-4.9 compare the success, crankback, and misscall ratios of the VPNs subscribing to the different abstraction topologies, and network utilization of the VSP for different load condition for Network-1. Figs 4.10-4.11 compare the call performance for Network-2 and Network-3.

With respect to Figs. 4.6-4.8, we observe that at lower load conditions, all VPNs including VPN-D which subscribes to a simple node abstraction and VPN-E that is not provided any abstraction are performing equally well; however, their performance deteriorates significantly with increasing load on the network. This is because, at low load conditions, the utilization of the network is less as seen in Fig. 4.9, and hence, the bandwidth requests from the VPNs (irrespective of the abstractions they were provided with and even for the case of no TA information) have their requests accepted by the VSP, which results in almost the same level of call performance. But with increasing load, that is, by increasing the mean call holding time $(H)$, an increasing number of calls remain in the network for longer time, which decreases the available bandwidth in the core; this results in increased contention for resources among the VPNs. This causes an increase in the crankback and misscall ratios and decreases performance in terms of success ratio.

We can see from Fig. 4.6 that the deterioration in success ratio is the most in the case of VPND-SNA and VPNE-NA (which is about $50 \%$ and $60 \%$ respectively), while VPNs with FMA, SSA, SA type of abstraction show only about $10 \%$ decline in the call performance metrics from their peak performance at lower loads. At the same time, we can also see that the performance of VPND-SNA with simple node form of abstraction 
performs better than the VPNE-NA case. This is because the remote access link state information updates (that are part of the simple node abstraction) enable VPN-D to make better routing decisions than the VPN-E which was provided with no link state information of the core network or the remote access link residual capacity information. VPN-E uses only the knowledge of its local access link capacity to decide if a call request can be forwarded to the adjacent PE node for path provisioning or not. Comparing FMA, SSA, and SA, FMA and SSA forms of abstractions show little difference in the performance with respect to success ratio. FMA performed only about $1 \%$ better than SSA at high load conditions. This implies that the presence of multiple virtual paths in a fully meshed abstraction does not prove to be of any particular advantage to the VPN. This observation is counter intuitive to what has been reported in a study [Hao00] related to topology aggregation for hierarchical routing in PNNI; but, this observation in [Hao00] may not hold well in the context of the TA service for the VPNs, which can be attributed to the following reasons.

Firstly, the path computation performed at the VPN end is only a feasibility check for the existence of a path satisfying the QoS requirement, in contrast to the case of hierarchical routing, where the path computation is also used to source route the call from the source domain to the destination domain. Secondly, the presence of multiple feasible paths in the FMA case in a VPN context may, in some cases, increase the probability of making erroneous decisions, with respect to availability of a feasible path in the core network. This is particularly true in situations of high load on the core network where all the virtual links of the FMA may not be in sync with the state of the core network. The 
results thus show that the increased overhead in terms of updates associated with fully meshed abstraction may not translate into significant advantage in terms of call performance as expected when compared to SSA case. Comparing FMA, SSA with SA, the performance of the SA was observed to be poorer by $5 \%$. However, considering that the deterioration in performance is not significant compared to SNA, it is clear that the average assignment (equations (3.1) \& (3.2)) of the virtual link capacity in the case of SA does prove to be a good approximation of the available bandwidth link metric of the core network.

The crankback ratio graph shown in Fig. 4.7 is negatively correlated to the success ratio discussed earlier. We observe that VPN-E and VPN-D with NA and SNA suffers from $50 \%$ and $40 \%$ crankback ratio respectively, while VPNs with either FMA, SSA or SA types of TA achieve a crankback ratio of less than $10 \%$. This gain in crankback ratio contributes towards good success ratio performance observed earlier.

We next discuss the relationship between misscall ratio performance shown in Fig. 4.8 and increasing load. Increasing offered load proportionally increases network utilization as shown in Fig. 4.9, hence the following discussion also holds good for misscall ratio performance versus increasing network utilization. From Fig. 4.8, we observe that the misscall ratio for VPNs with either fully meshed, source-star, or star abstraction first decreases with increasing load and then increases at high load. This is due to the following reason. As the load increases from low to medium range, the probability of the TA being out of sync with the link state of the core network increases. This, in turn, increases the probability of a VPN making wrong decision of wrongly 
terminating the call locally, thereby resulting in increase of misscall ratio. At high loads, the residual link capacity for most of core links is less because of higher network utilization as shown in Fig. 4.9. This results in lesser virtual capacity to be exposed to the VPNs for all the three abstractions, which improves the probability of the VPN TA to be synchronous with the core state of the network. This increases the VPN's success rate of rejecting a path request locally correctly, resulting in improvement of misscall ratio performance at high load conditions. The same reasoning is also true for the nature of success ratio and crankback ratio graphs for FMA, SSA, and SA cases in Fig 4.6 and Fig. 4.7. From the Fig. 4.8, we observe that VPN-A and VPN-B with FMA and SSA suffer a maximum misscall ratio of $7 \%$, while VPN-C with SA performs poorly compared to FMA and SSA with a maximum misscall ratio of $11 \%$. We also observe that VPN-D with SNA achieves a misscall ratio of less than $1 \%$, while VPN-E with NA has $0 \%$ misscall ratio (technically misscall ratio does'nt apply for the NA case). A marginal degradation of misscall ratio is the tradeoff made by the VPNs subscribing to the TA service in return for very good crankback and success ratio performance discussed earlier.

Figs. 4.10 (a-b) compare the performance of the VPNs with respect to success ratio and (crankback + misscall ) ratio for Network-2. The performance trends for the call performance metrics in this case are similar to that observed for Network-1; particularly in terms of the performance difference between the VPN with NA and the VPNs with FMA, SSA, or SA forms of TA. One important observation in the case of Network-2 is that even VPN-D with SNA performs better than what was observed in the case of Network-1. This can attributed to the size of network topology of Network-2 (11 nodes) 
compared to Network-1 (22 nodes). In general smaller topology has less network capacity and fewer number of paths in the network between any pair of border nodes. This improves the crankback and misscall (Fig. 4.10 (b)) performance considerably for the SNA case for Network-1 compared to Network-2. Hence, a VPN subscribing to SNA tends to be penalized more when it is connected to a larger VSP network topology than a smaller VSP network topology.

Figs. 4.11(a-b) compares the performance of the VPNs with respect to success ratio and (crankback + misscall) ratio for Network-3. The performance characteristic of the VPNs in this case is very similar to the one made in the context of Network-1 for success, crankback and misscall ratio performance metrics.

To conclude, these results demonstrate the usefulness of the TA service in a dynamic bandwidth request scenario, particularly, at high network load conditions. We observed that VPNs with NA and SNA had their performance deteriorate significantly in terms of success and crankback ratio. For VPNs with either FMA, SSA, or SNA forms of abstraction, the significant improvement of the crankback ratio resulted in very good performance in terms of success ratio at the cost of marginal increase of misscall ratio. We also observed that among FMA, SSA and SA, SA performed relatively poorly, while FMA and SSA did not show any obvious difference in performance. We study this point further in the next objectives. Before we end the study of this objective, we study the performance of the abstractions in terms of fairness and network utilization.

Just as in the case of objective 1 , the performance characteristic trends for the remaining objectives were observed to be similar for all the three topologies; hence in 
order to avoid redundancy, we limit our discussion for the remaining objectives for the case of Network-1.

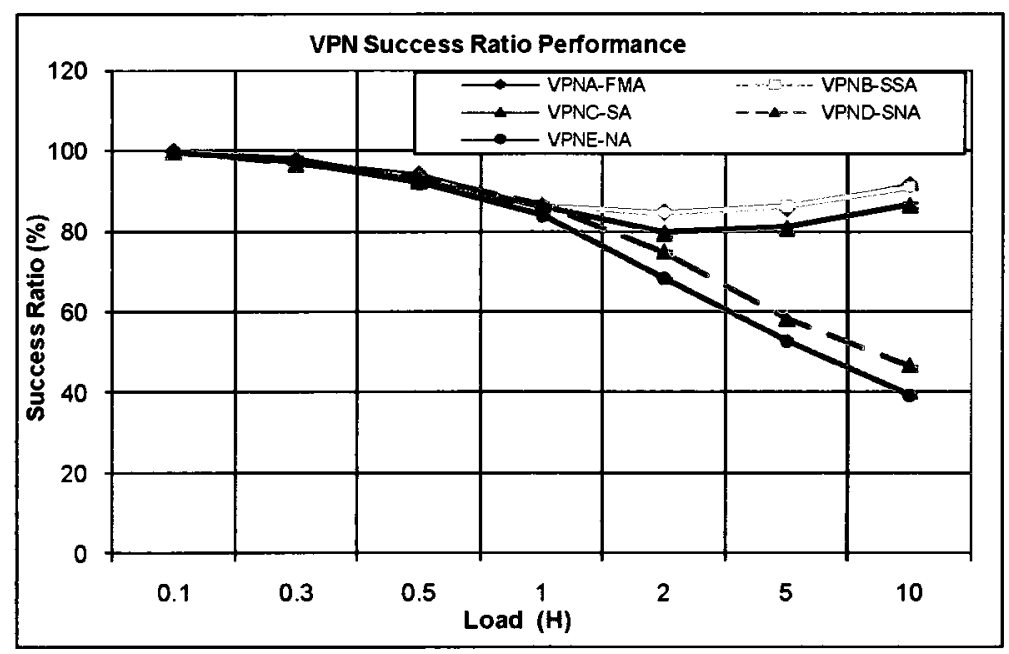

Figure 4-6: Objective 1, VPN call performance comparing success ratio with varying mean holding time (H) with $95 \%$ C.I (Network-1)

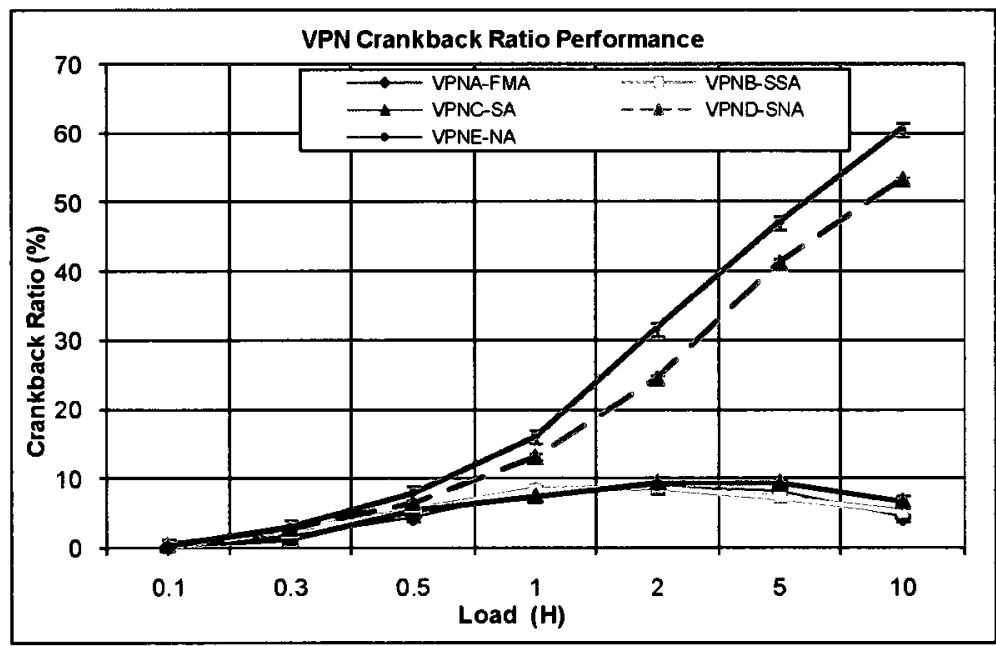

Figure 4-7: Objective 1, VPN call performance comparing crankback ratio with varying mean holding time (H) with $95 \%$ C.I (Network-1) 


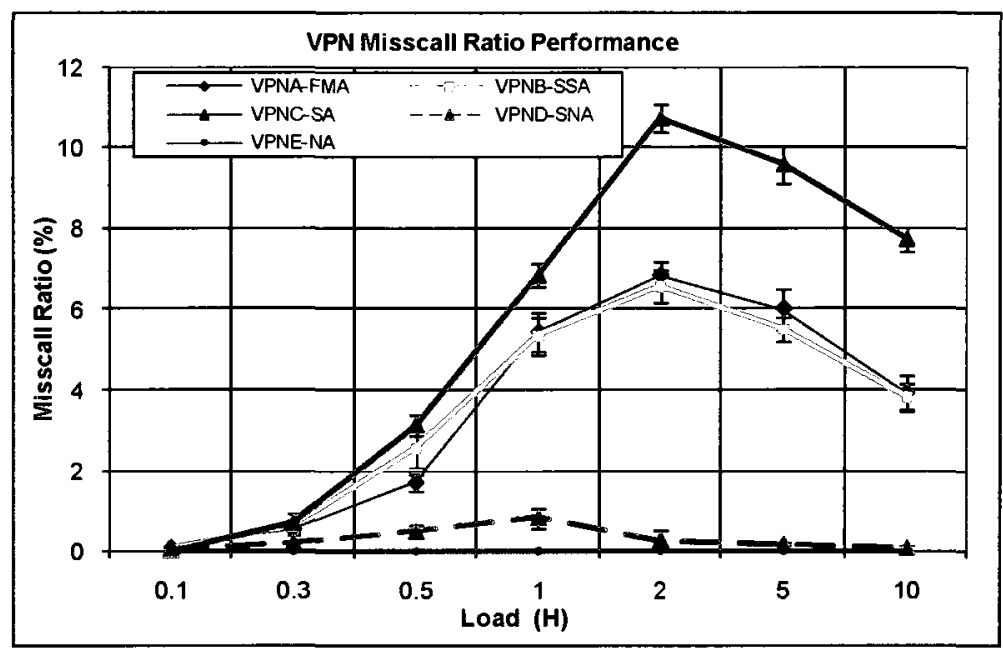

Figure 4-8: Objective 1, VPN call performance comparing misscall ratio with varying mean holding time $(\mathrm{H})$ with $95 \%$ C.I (Network-1)

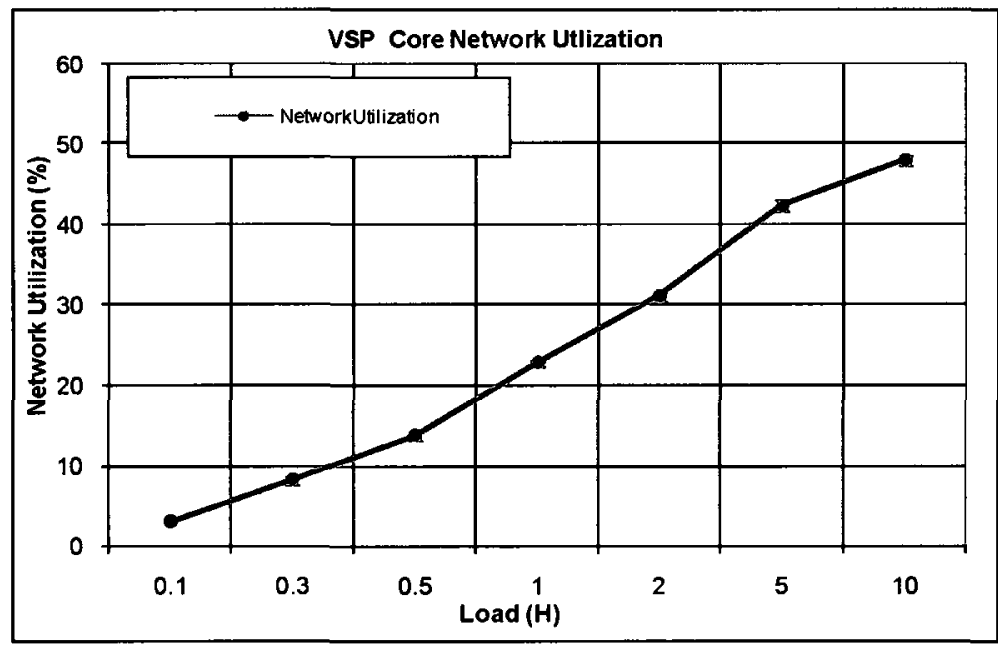

Figure 4-9: Objective 1, VSP core network utilization with varying mean holding time (H) with 95\% C.I (Network-1) 

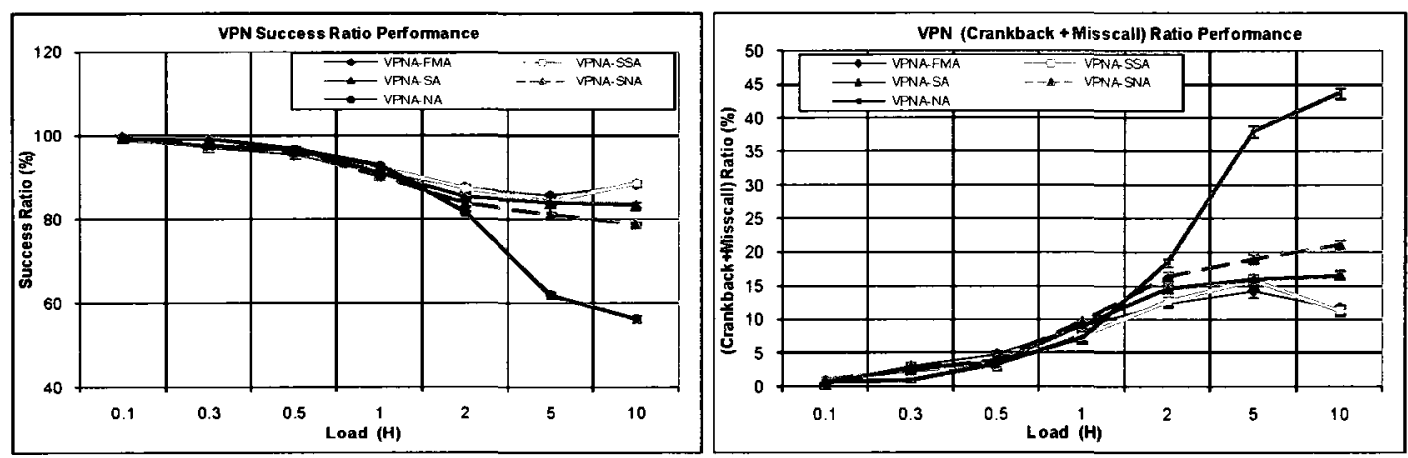

Figure 4-10 (a-b): Objective 1, Comparison of success ratio and (crankback + misscall ratio) with varying mean holding time (H) with $95 \%$ C.I (Network-2)
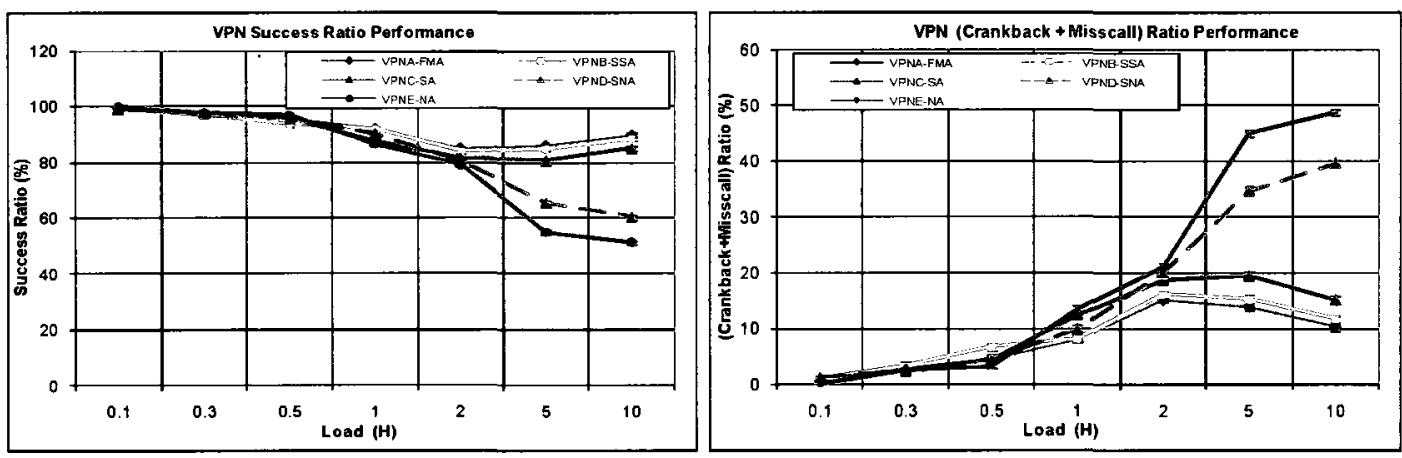

Figure 4-11 (a-b): Objective 1, Comparison of success ratio and (crankback + misscall ratio) with varying mean holding time $(\mathrm{H})$ with $95 \%$ C.I (Network-3)

\section{VPN Fairness and Core Network Utilization}

Here, we study how the policy of equally exposing available capacity to all the VPNs with the same $T_{k}$ parameter enforces faimess in terms of performance metrics for all the VPNs. We also discuss how the various forms of abstractions affect network utilization.

To study the performance with respect to faimess, we set the TA subscription type of all the VPNs to be of type source-star type (SSA). In this case, we would expect all the VPNs to be exposed the same capacity over their virtual links, and hence, the variance in performance is expected to be as minimum as possible for the same mean call arrival rate 
and holding time scenario. The fairness is quantified by the standard deviation of the performance metrics for the five VPNs.

Fig. 4.12 shows the standard deviation (SD) of success, crankback, and miscall ratios for all the VPNs with increasing load. We observe that the SD of the performance metrics of the five VPNs is less than $2 \%$, which indicates that the model of sharing resources equally results in the desired objective of achieving fairness to all the VPNs. We also observe that SD increases slightly at higher loads. This is expected because of increasing resource contention and lack of synchronization between the core topology information and the abstractions provided to the VPNs.

Fig. 4.13 compares the effect of the choice of abstract topology type parameter on core network utilization. Here, the statistics were collected for multiple simulation scenarios; for each scenario all the five VPNs in a run are set to one of FMA, SSA, SA, SNA abstraction types. In addition comparison is also shown for the case when VPNs are not provided with any topology information; this case corresponds to the NA type in the results. From the graph, we can observe that the scenario with no abstraction and simple node abstraction resulted in $1 \%-3 \%$ better network utilization than the VPNs with some form of TA; however, as we discussed earlier, this gain is at the cost of significant deterioration in terms of the call performance metrics. The better performance in terms of network utilization achieved by the SNA scenario is expected, as this abstraction is not provided any information about the link state of the core network, resulting in performance similar to the NA case, but with improved call performance statistics. We can also observe that, VPNs which were provided one of FMA, SSA achieved almost the 
same level of performance in terms network utilization; while VPN with SA resulted in network utilization which is $4 \%$ poorer than the FMA and the SSA case. The poorer performance of the SA case correlates with performance degradation of $5 \%$ observed with respect to the call performance metrics as discussed earlier. From the above observations, we can conclude that providing TA service, while enabling very good call performance to the VPNs, doesn't results in decrease of core network utilization.

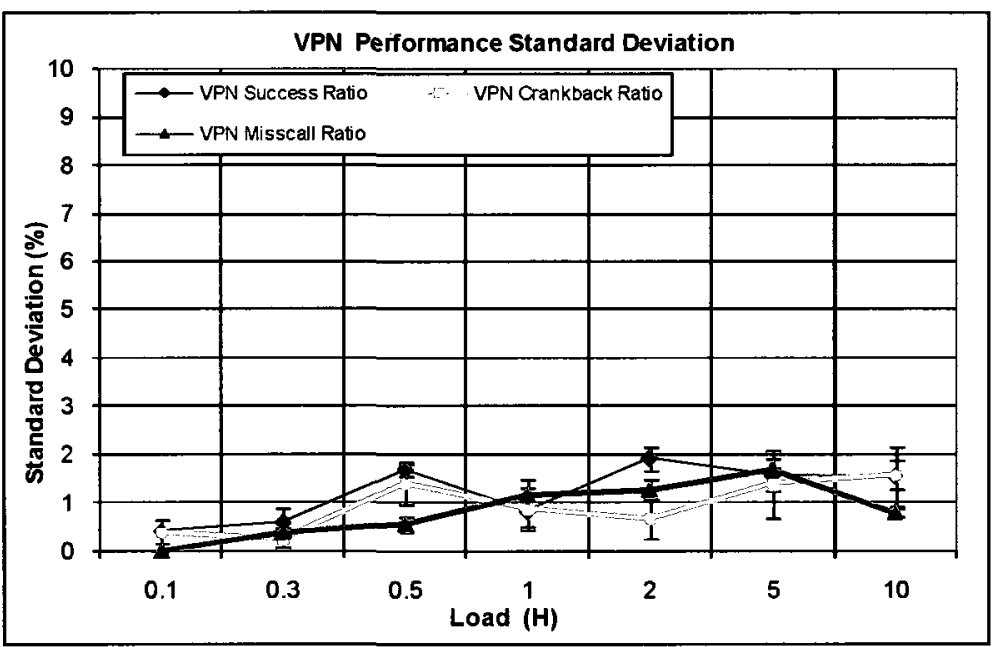

Figure 4-12: Objective 1, Fairness performance in terms of standard deviation of call performance metrics with varying mean holding time (H) with $95 \%$ C.I (Network-1) 


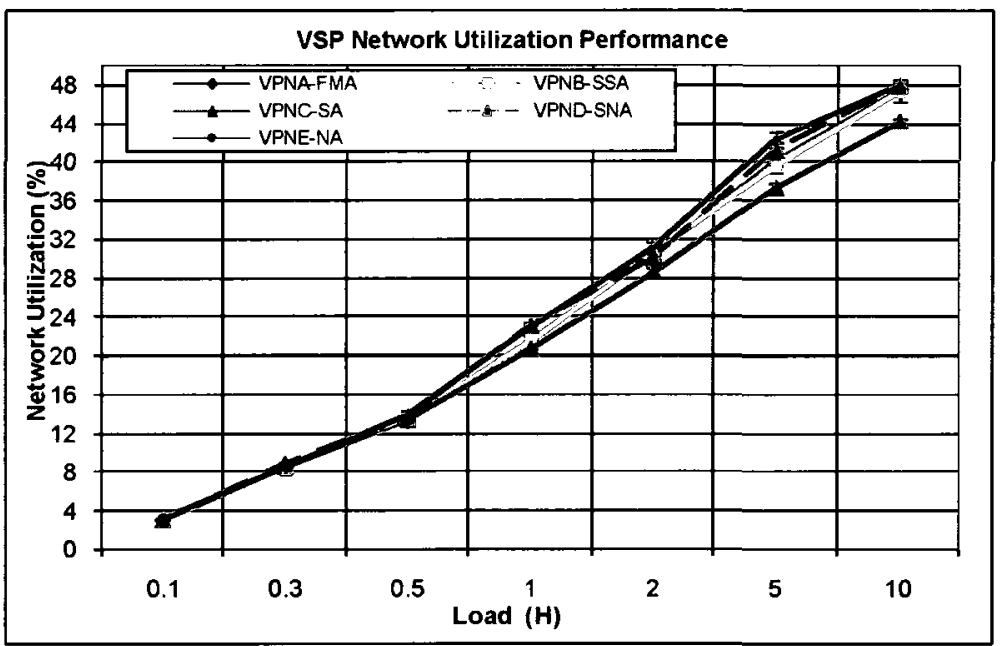

Figure 4-13: Objective 1, Comparing VSP network utilization for different abstraction types with varying mean holding time (H) with 95\% C.I (Network-1)

\section{Objective 2: VPN Abstract Topology Refresh Interval $R_{k}$ as a service parameter}

In this scenario, we study if $R_{k}$ can be used as a service differentiating parameter. For this, the network load is held constant at 5 Erlang, while the $R_{k}$ of the VPNs is varied between $[20,1000] \mathrm{s}$. The bandwidth requests from the VPN is uniformly distributed in the range of $[1,500]$ units.

Figs. 4.14-4.15 illustrate the effect of increasing the abstract topology update interval. The graph in Fig. 4.14 shows the performance in terms of the sum of crankback and misscall ratios, which has to be minimum to imply good performance. The results show that with increasing $R_{k}$, the success ratio decreases while crankback ratio, and misscall ratio increases for each of the topology abstractions, which can be attributed to increasing lack of the latest state of the core network information with the VPN CE nodes.. Observing the maximum and minimum performance level from the graphs for 
each of the abstraction types in Fig. 4.14, we see that the performance of the VPN with FMA drops by $24 \%$, while it drops by $25 \%$ for the SSA and SA case. SA also performs poorly compared to FMA and SSA by $3 \%$ over the range of refresh interval values. This shows that FMA and SSA does prove to be of some advantage with increasing abstract topology refresh interval. Similar deterioration in performance is also noted with respect to crankback and misscall ratio in Fig. 4.15 among the three forms of abstractions. From the graphs we also observe that, the performance of SA compared to FMA and the SSA case is not significant. This behaviour could be attributed to the averaging used while assigning bandwidth to the virtual link in a star abstraction that offsets the lack of the latest network state information with the VPN.

This analysis doesn't prove the desired property of using abstract topology refresh interval as a parameter to generate significant service differentiation between the three forms of TA. However, from the deteriorating call performance characteristic with increasing abstract topology refresh interval metric, we can state that, for a given set of VPNs with the same $T_{k}$ parameter, different settings of $R_{k}$ parameter can be used to create service differentiation. 


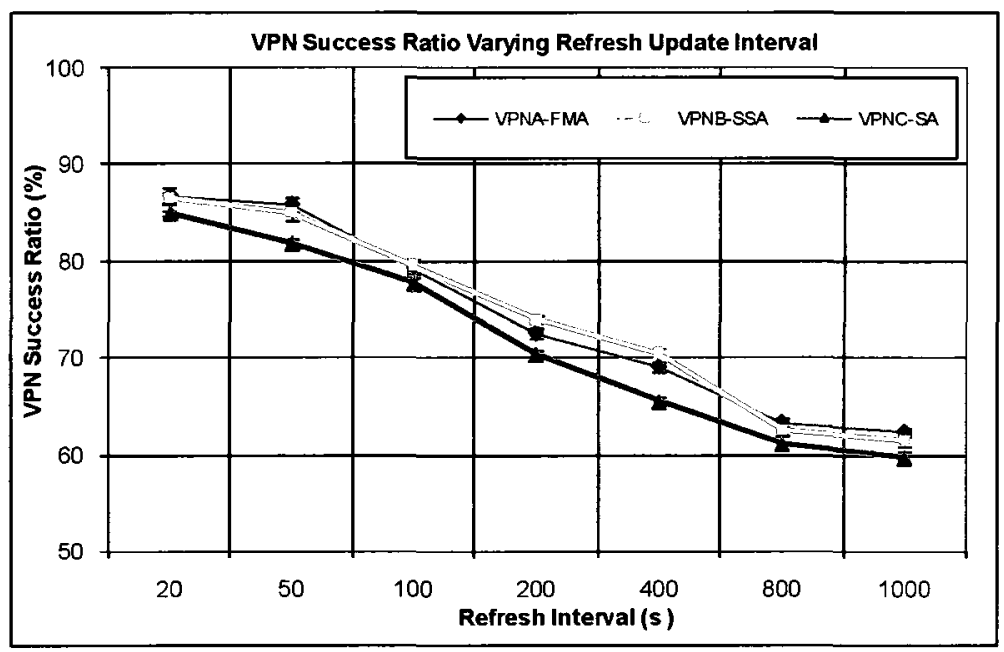

Figure 4-14: Objective 2, VPN call performance comparing success ratio with varying refresh update interval with $95 \%$ C.I (Network-1)

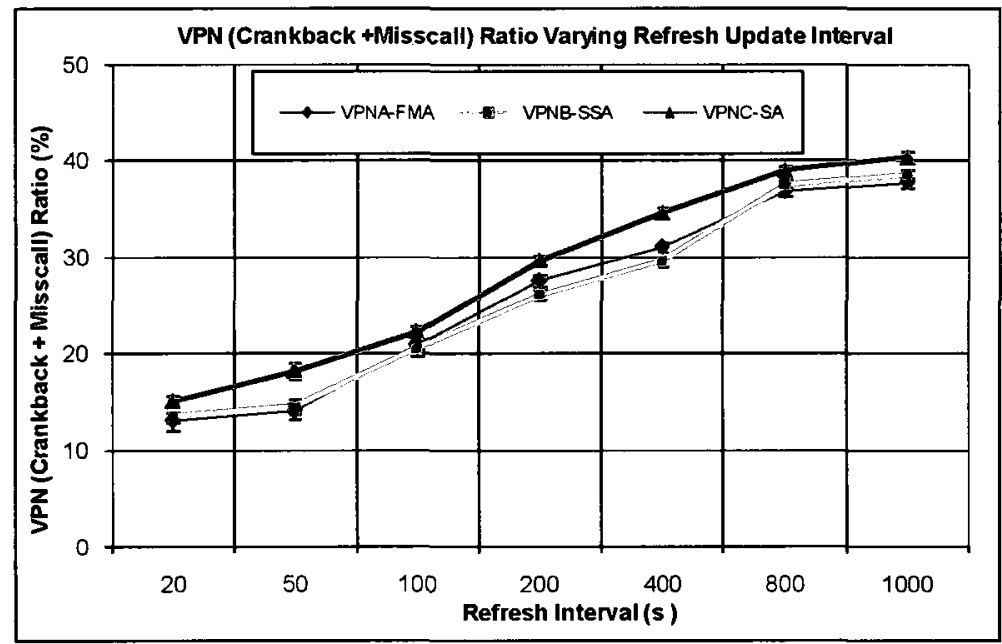

Figure 4-15: Objective 2, VPN call performance (crankback+ misscall) ratio with varying refresh update interval with $95 \%$ C.I (Network-1)

\section{Objective 3: Combining Abstraction Granularity with varying Refresh Interval}

In this scenario, we study the combined effect of using $T_{k}$ with $R_{k}$ as service parameters to create service differentiation. We do this to widen the gap of call performance between more granular abstract topologies, like fully meshed, and lesser granular abstractions, like 
source-star and the star forms of abstraction. In all the above objectives that were discussed earlier, the assumption was that all the VPNs are treated equally, with respect to correctness of the core state information, and hence, the same value of $R_{k}$ was set for all the VPNs irrespective of the $T_{k}$ parameter setting. To study this objective, the call parameters from objective 1 were retained, but different abstract topology refresh intervals were set for different abstraction schemes. We set the VPN update interval for fully meshed abstraction to $50 \mathrm{~s}$, source-star abstraction to $250 \mathrm{~s}$, and VPN subscribing to star abstraction scheme to $750 \mathrm{~s}$.

Figs. 4.15-4.16 compare the performance of VPNs in terms of success ratio, crankback, and miscall ratio with these settings. From these results, we observe that at lower loads the low utilization of the core network resources offsets the effect of variable $R_{k}$ setting for different TA types; but with increasing load, inaccurate information because of higher refresh update interval setting in case of VPNB-SSA and VPNC-SA causes it to perform poorly compared to VPNA-FMA, resulting in call performance differentiation between the three abstractions. The higher value of $R_{k}$, in cases of SSA and SA compared to FMA abstraction, resulted in higher number of call crankbacks and miscalls, hence poorer success ratio performance than the fully meshed scheme. From the graphs we observe that at the maximum load, FMA out performs SSA and SA by $10 \%$ and $15 \%$ respectively, while SSA outperforms SA by $5 \%$. The nature of the graphs is so for the same reasons as discussed in objective 1. Thus, this scenario shows that the two service parameters $T_{k}$ and $R_{k}$ could be effectively combined in order to provide a differentiated topology abstraction service with varying call performance characteristics. 


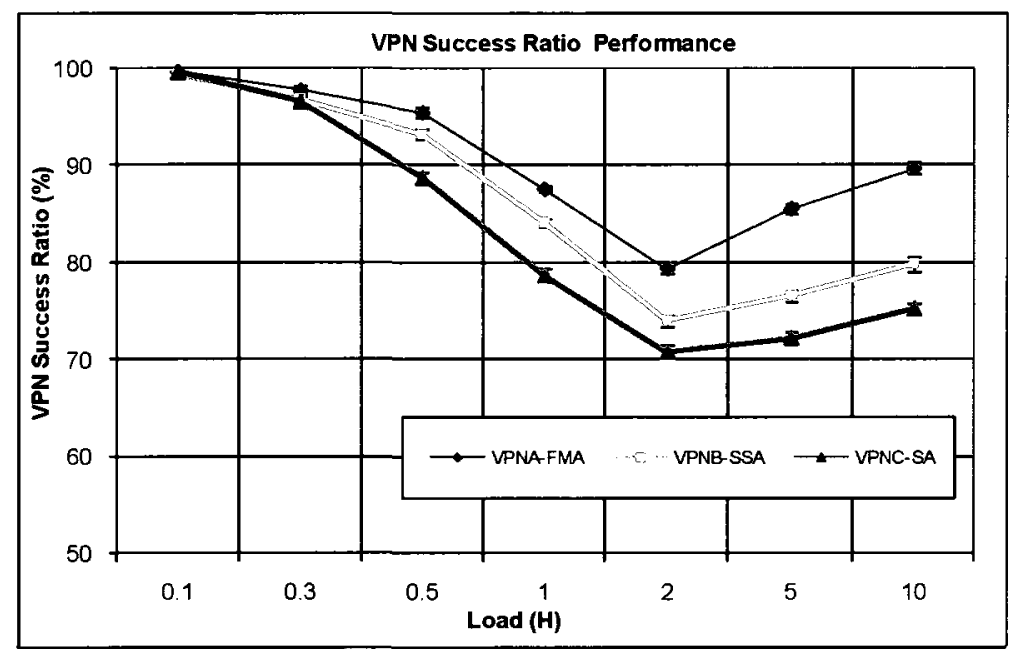

Figure 4-16: Objective 3, VPN success ratio with varying refresh update interval and varying abstraction type with varying mean holding time (H) with $95 \% \mathrm{C}$.I (Network-1)

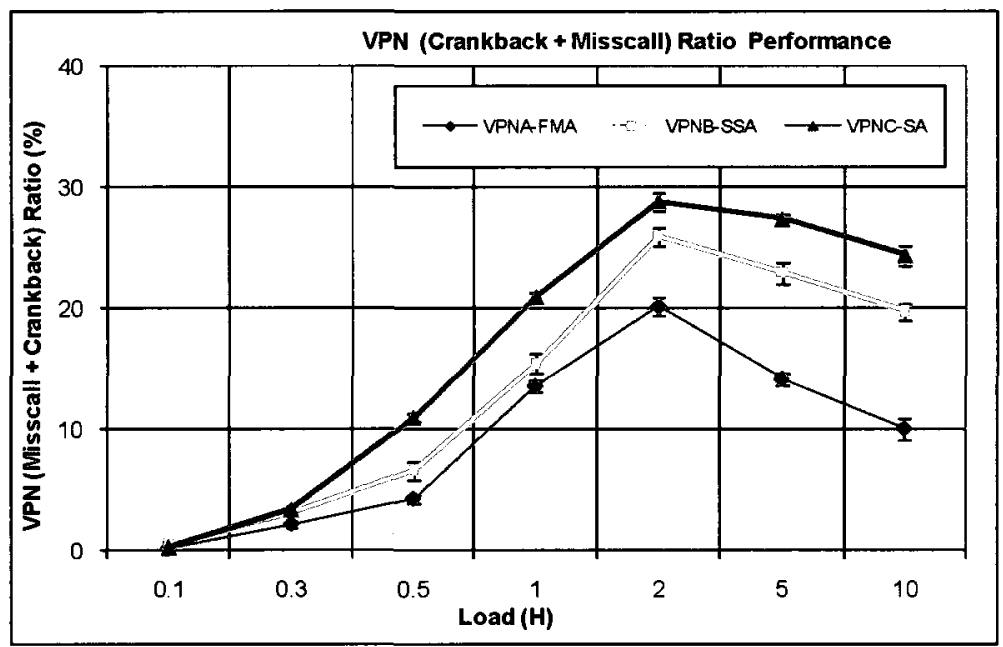

Figure 4-17: Objective 3, VPN (crankback + misscall) ratio with varying refresh update interval and varying abstraction type with varying mean holding time (H) with $95 \%$ C.I (Network-1) 


\subsection{Discussion}

Having studied the performance of the TA service for the various abstraction types, we next present a discussion on the usefulness and drawbacks of using TA service and mitigation of the effects of these drawbacks.

The usefulness of TA service to the VSP and the VPN is as follows:

- When a VPN subscribes to the TA service, VPN gains in terms of very good success ratio. This achieves the objective of improving a VPN's routing efficiency significantly. Also, the VPNs don't face the situation of getting rejected a high percentage of its path requests particularly at higher load conditions, resulting in good VPN experience. For instance, for all the three network topologies considered for the simulation, even at higher network load conditions, the success ratio for VPNs with either FMA, SSA or SA is more than $80 \%$ compared to less than $50 \%$ for the noabstraction case.

- One of the reasons for consistent success ratio performance of the VPNs provided with TA information at different load conditions is because of the significant gain in crankback ratio. This has signalling cost advantage to the VSP. In current provider networks control plane scalability is a major concern. Significant gain in the correct termination of the infeasible path requests reduces the amount of path requests required to be processed by the VSP. This makes valuable control plane processing resources to be available for other purposes. This signalling cost saving increases with increase in number of VPNs hosted on the border nodes. 
The drawbacks of applying the TA service are as follows:

- We observed that, at high loads, the VPNs with TA resulted in increase in misscall ratio compared to the case when no TA is employed. However, the deterioration is smaller compared to the significant gain in terms of success ratio. For instance, we saw from the simulations for Network-1, that the miscall ratio for the VPN provided with star abstraction (which was the worst performer) is less than $11 \%$ (Fig. 4.8). Fully meshed and source-star abstraction performed $4 \%$ better than star abstraction. However, this increase in misscall ratio is insignificant compared to $40 \%$ performance difference in terms of success ratio (Fig. 4.6) achieved by the VPNs provided with TA compared to the no-abstraction case.

- We also observed a difference in performance in terms of network utilization when TA service was and wasn't applied. This is a result of increase in misscall ratio as discussed in the previous point. However, this degradation is a marginal cost incurred by the VSP in light of the significant efficiency gained in terms of good routing performance achieved by the VPNs. For instance, for Network-1, the maximum difference in network utilization compared to the no-abstraction case was observed to be about 3\% (Fig.4.13). This is for the case when all VPNs were provided with star abstraction.

- The VSP bears a control overhead associated with generating the TA and sharing it with the VPNs. This scales in the order of $|B|^{2}$ for fully meshed abstraction and linearly as $|B|$ for source-star, star, and simple node abstractions $(|B|$ is the number of border nodes in the VSP's network) for each VPN. 
Mitigation for the above drawbacks:

- The above drawbacks can be mitigated by choosing the appropriate TA-SLA metrics by the VPN. From the simulations we observed that different TA types resulted in different misscall ratio performance, which also influenced the network utilization. FMA and SSA performed the best in terms of misscall ratio while SA performed the worst. This difference in performance based on TA granularity, allows a VPN to choose an abstraction that meets its desired criteria with respect to misscall ratio.

- The control overhead complexity can be mitigated by controlling the amount of information flooded from the PE to the CE node which is also at the discretion of the VPN. This can be realized by choosing a lower granular topology abstraction type instead of a more granular one or by choosing a higher abstract topology refresh interval instead of a lower one. This will allow the VPN to manage the TA information in an efficient manner.

\subsection{Summary}

In this chapter, we began our discussion on the two modes of TA generation in the context of the TA service to the IP-VPNs, i.e. centralized and decentralized ways of TA generation. We then discussed the three-stage process of generating TAs for the VPNs to satisfy the TA-SLA requirements and the fairness definition. This process guarantees that all the VPNs subscribing to the same topology abstraction will be exposed equal virtual link capacities and result in similar call performance, while allowing them to receive different levels of service if they subscribe to different topology abstractions. 
As part of our validation of the proposed ideas of providing TA service to the IPVPNs in the context of the MDVF, using simulation analysis, we showed that providing even minimum topology information such as simple node abstraction improved the call performance scenario under dynamic bandwidth request scenario when compared to the current approach adopted in IP-VPN solutions where no topology or link stage information is provided. This difference was particularly noticeable at high load conditions. VPNs with fully meshed, source-star and star abstractions performed significantly good in terms of success ratio. We also noted that with TA service the VPNs suffered from misscall ratio and reduction of network utilization compared to the noabstraction case.

The simulation results showed that among the different forms of abstract topologies, the simple node scheme suffers from poor call performance in comparison to the star, source-star, and the fully meshed abstraction types. The star abstraction faired relatively poorly compared to almost equally performing source-star and fully meshed abstraction. We also observed that the performance of fully meshed, source-star, and star did not show a significant difference when the abstract topology refresh interval metric was varied over a range of values. However, VPNs subscribing to the same abstract topology type could be set to different refresh update periods in order to create service differentiation. In addition, from another simulation scenario, we also observed that the abstract topology refresh interval, in combination with an abstraction topology type parameter, could also be used to create a more explicit service differentiation between 
VPN customers subscribing to either fully meshed, source-star, or star forms of abstraction. 


\section{Chapter 5}

\section{Decentralized VPN Topology Abstraction}

\subsection{Introduction}

The TA service to IP-VPNs basically involves sharing and abstracting available core resources among multiple VPNs, which can use it to seek resources on demand. In the previous chapter, we examined the problem of generating abstract topologies in a fair manner without addressing the oversubscription issue encountered in a decentralized mode of TA generation. Generating TAs without considering this issue could result in poor call performance and network utilization. This has motivated us to investigate into better algorithms for the decentralized mode of TA generation. For this, we begin Section 5.2 by understanding the reasons for oversubscription and defining a problem called the VPN topology abstraction (VPN-TA) problem to address this issue. As a solution to this problem, three topology abstraction schemes are proposed. Each of these proposed TA schemes has a tradeoff in terms of VPN call performance and network utilization, and thereby, enabling the providers to choose a scheme that meets their specific objectives. Section 5.3 evaluates the three abstraction schemes via the simulation analysis. 


\subsection{VPN Topology Abstraction Problem}

The reason that motivates the need to define this problem is as follows: the process of generating TAs as part of the TA service for the VPNs in a decentralized manner may result in oversubscription of the available core resource, which could result in poor VPN call performance and network utilization during high load conditions.

Oversubscription results because of two reasons: first, as noted earlier, the decentralized approach of TA generation where in each border node generates TAs for the VPNs without coordinating with one another; second, the degree of oversubscription also depends on the abstraction algorithm that is used to generate the abstraction for each VPN by the border node. For instance an abstraction algorithm might expose more capacity than what can be satisfied between a pair of border nodes which results in a situation of contention for resources among VPNs resulting in poor call performance. The objective of defining the VPN-TA problem is to address the problem of oversubscription arising due to the second factor. For this we begin by illustrating by an example the issue of oversubscription due to overexposure of available capacity to the VPNs.

Consider the example shown in Fig. 5.1, where the VSP provides TA service to two VPNs subscribing to fully meshed type of abstraction generated using the methodology discussed in Section 4.2 (Chapter 4). The resulting abstractions for the red and blue VPNs are shown in Figs. 5.2-5.3. In these figures the variables associated with the virtual links represent the capacity exposed to the VPNs. A variable notation for the exposed capacity in the abstractions has been considered in order to explain the notion of 
oversubscription in a general context and to avoid making any particular assumption on the abstraction algorithm that is applied to compute the virtual link capacity metric.

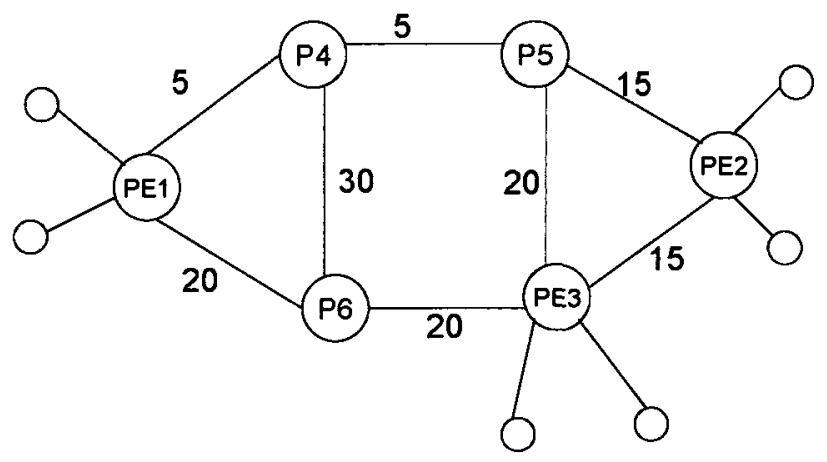

Figure 5-1: VSP providing topology abstraction service to red and blue VPN

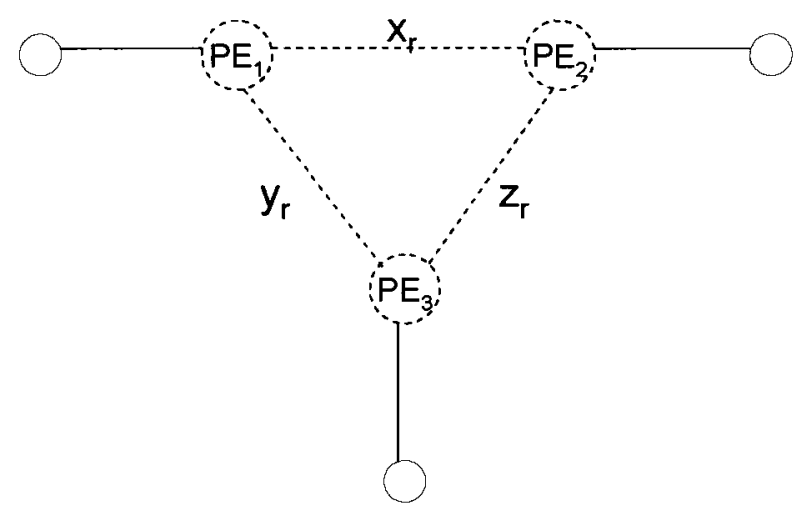

Figure 5-2: Fully meshed abstraction for red VPN

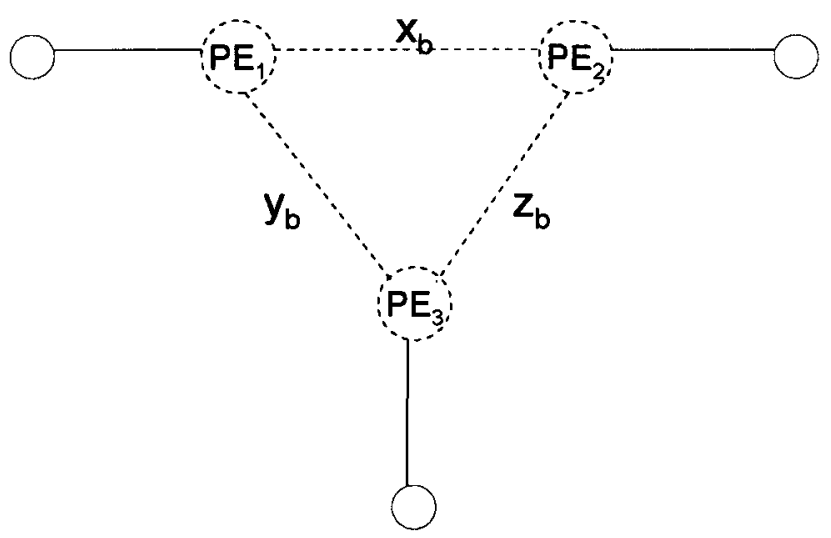

Figure 5-3: Fully meshed abstraction for blue VPN 
To explain the problem of oversubscription, we consider all possible disjoint paths between border nodes PE1 and PE2. From Fig. 5.1 we see that there are two disjoint paths between PE1 and PE2, which are $\mathrm{P}_{1}=\{\mathrm{PE} 1 \rightarrow \mathrm{P} 4 \rightarrow \mathrm{P} 5 \rightarrow \mathrm{PE} 2\}$ of capacity 5 , and $\mathrm{P}_{2}=\{\mathrm{PE} 1 \rightarrow \mathrm{P} 6 \rightarrow \mathrm{P} 3 \rightarrow \mathrm{PE} 2\}$ of capacity 15 which results in maximum available capacity of 20 units. Oversubscription occurs if the sum of the exposed capacities corresponding to the virtual links between PE1 and PE2 in the TA for the two VPNs exceeds the total available capacity of 20 units i.e $x_{r}+x_{b}>20$. In such a situation, during high load conditions there is a good possibility that the requests from the blue and red VPNs could have close arrival times at the border node, in which case one of the calls will be rejected. An example of this is when the blue VPN request has been successfully provisioned and a request from the red VPN arrives at the border node before it has been updated about the current state of the network; this results in the PE node rejecting the call request from the red VPN. In a more general case with many VPNs and under high load condition, such a situation of call arrivals occur more often, causing the border node handling the requests to crankback one or more VPN requests. In order to address this situation, care must be exercised in Step 2 and Step 3 of the TA generation process (Fig. 4.1), where the capacity of the virtual links of the fully meshed abstraction are computed. Thus, our objectives while generating a TA abstraction are as follows.

- Provide the VPNs with a reliable representation of available capacity considering the case of having to satisfy VPN calls during high load condition. This objective also correlates with the objective of maximizing the call performance of the VPNs. VPN call performance is also related to whether the abstraction schemes 
used to generate the TAs are optimistic or conservative in terms of exposing the available core capacity to the VPNs. Optimistic abstraction schemes tend to oversubscribe the available resource with the goal of maximizing statistical multiplexing of the core capacity which could lead to poor call performance. On the other hand conservative TA schemes tend to minimize oversubscription by exposing only the capacity that can be satisfied assuming the worst case of receiving several call requests simultaneously, leading to a situation where the VPNs may terminate the calls locally as a result of the route computation locally even when capacity exists in the core to satisfy the call requests. Hence, the proposed TA generation scheme has to achieve a balance between the two types of TA generation methods.

- The other objective is to maximize network utilization of the core network. The VPNs use the abstract topology information provided by the VSP to decide the feasibility of a call request in the context of the TA service. Hence, the algorithm used to generate the TA should not only enable the VPNs to make the correct decision on availability of resource in the core but also be efficient to maximize its usage. The maximization of the network utilization is also related to the previously discussed factor of whether the abstraction is optimistic or conservative in nature.

- The third objective of the TA generation schemes should be the ability to generate fair abstraction which aligns with the fairness policy stated in Section 4.2.1 in the context of the TA service fairness requirement for the VPNs. 
The VPN TA abstraction problem defined next takes into consideration the above factors: maximization of call performance, fairness to all VPNs in allocating resources, and maximization of network utilization.

\section{VPN Topology Abstraction (VPN-TA) Problem:}

Given a set of VPNs $U_{b}$ hosted on the border node $b$, each VPN instance $i \in U_{b}$ is to be provided an abstract topology $G_{i, l}\left(V_{i}, E_{i}\right)$. The objective of the problem is to device a methodology to allocate virtual capacities to the links in $E_{i,}$, so that the VSP maximizes the probability of each VPN making a correct decision of successfully computing or rejecting a path locally in the context of the TA service.

We propose three algorithms for the above problem. With reference to the steps discussed in Section 4.2, these schemes are executed as part of Step 2 and Step 3 in the abstract topology generation process (Fig. 4.1). In these steps the abstraction schemes computes partition subgraphs $S\left(V_{k}, E_{k}\right)$ and generates fully meshed abstraction $G_{k, F M A}\left(V_{k}, E_{k}\right)$ for VPN $k$. The first abstraction approach exposes the maximum capacity available between two border nodes to a VPN. The other two approaches are called the mixed bound approach and Steiner tree graph approach. As the VPN-TA problem is defined in a decentralized context, the algorithms presented in the following sections are in the context of a PE node $b$ which executes these algorithms as part of the TA generation 
process. The pseudo code presented for each abstractions scheme follows the abstraction generation process presented in Fig. 4.1 in Section 4.2.

\subsubsection{Maximum Capacity Abstraction Scheme}

This is an aggressive approach to generate VPN abstract topologies. In this scheme, the VSP sets the virtual link capacity corresponding to a pair of border nodes of a fully meshed abstraction to the capacity of the widest path (defined next) between the two nodes. For a given pair of border nodes $\left(b_{1}, b_{2}\right) \in P_{k}$ hosting a VPN $k$, let $P=\left\{p_{1}, p_{2} \ldots . p_{l}\right\}$ be the set of paths between the two nodes. Let $C\left(p_{i}\right)$ be the bottleneck capacity of each path. We define $\omega\left(b_{1}, b_{2}\right)=\max _{i=1 \ldots l}\left(C\left(p_{i}\right)\right)$ as the maximum capacity of a path corresponding to the pair of border nodes $\left(b_{1}, b_{2}\right)$. This path is called the widest path between the pair of nodes. Once the capacity of this path is computed, we set the virtual link capacity $w_{k, F M A}\left(b_{1}, b_{2}\right)$ of VPN $k$ to $\omega\left(b_{1}, b_{2}\right)$.

We can obtain the widest path capacity by computing a maximum capacity tree rooted at border node $b$. We refer to this tree as the partition graph $S\left(V_{\mathrm{k}}, E_{k}\right)$ for VPN $k$ rooted at $b$. This tree spans all the nodes of $P_{k}$. This tree can be obtained in $O\left(|V|^{2}\right)$ time by applying a modified form of the Dijkstra's shortest path algorithm [Ahu93] (Appendix A.1). A tree thus computed enables one to generate the set of virtual links from node $b$ to other nodes in $P_{k}$. However, since the goal is to generate the set of widest path capacities of the virtual links of a fully meshed abstraction, a border node $b$ will also have to compute partition graphs $S\left(V_{k}, E_{k}\right)$ rooted at all the border nodes in $P_{k}$, by iterating the partition graph computation step for all nodes in $P_{k}$. Once the fully meshed abstraction 
for a VPN is computed, this subgraph computation step further proceeds to compute the fully meshed abstractions for other VPNs in $U_{b}$. The worst case complexity for a border to compute the fully meshed abstraction, assuming that every border node hosts all VPNs in $U$, is $O\left(|U|^{*}|B|^{*}|V|^{2}\right)$. As noted earlier, this approach is very aggressive since the maximum capacity between a pair of border nodes is advertised to all the VPNs having that pair of border nodes in common. This aggressive mode of capacity sharing may work well during low load conditions, during which there is a high probability that the capacities associated with the virtual links are in sync with the state of the core network. However, may result in poor call performance at high load conditions when the abstraction at the VPNs end may not be synchronized with the state of the core network.

The pseudo code used in generating the abstract topology using this scheme by a border node $b$ for the set of VPNs $U_{b}$ is shown in Fig. 5.4 in line with the TA generation process shown in Fig. 4.1. In this algorithm, applying the fairness Steps 4-6 (Stage 2) of Fig. 4.1, could be skipped, since the algorithm assigns the widest path capacity to all the virtual links for all the VPNs that have a pair of border nodes in the fully meshed abstraction computed in Step 3, and in this way, satisfying the fairness criteria defined in Section 4.2.1. 


\section{Maximum Capacity Path Algorithm}

Input: $G(V, E), U_{b}, T S_{k} \forall k \in U_{b}$.

Output: $G_{k, l}\left(V_{k}, E_{k}\right), \forall k \in U_{b}$ and $l=T_{k}$.

begin

//Stage 1: Generate partition graphs and fully meshed abstraction

\section{begin}

(Iterate the remaining steps for each VPN $k \in U_{b}$ for which abstract topology is being computed. We determine the capacities of virtual links of the fully meshed abstract topology as follows).

Step 1: identify the set of PE nodes $P_{k}$ belonging to VPN $k$;

//initialize graph $G_{k, F M A}\left(V_{k}, E_{k}\right)$

for all $x, y \in V_{k}$, set $w_{k, F M A}(x, y)=0$; (Note: For the fully meshed case the set $V_{\mathrm{k}}$ in $G_{k, F M A}\left(V_{k}, E_{k}\right)$ is same as $P_{k}$.

Step 2: compute the widest path tree $S\left(V_{\mathrm{k}}, E_{k}\right)$ rooted at each border node $x, \forall x \in P_{k}$ and spanning all the nodes in $y \in P_{k}$ and $y \neq x$;

//Generate $G_{k, F M A}\left(V_{k}, E_{k}\right)$ using $S\left(V_{k}, E_{k}\right)$ rooted at each $x \in P_{k}$

Step 3: for each $(x, y) \in E_{k}$ in $G_{k, F M A}\left(V_{k}, E_{k}\right)$, set $w_{k, F M A}(x, y)=\omega\left(S\left(V_{\mathrm{k}}, E_{k}\right), x, y\right)$; (Note: $\omega\left(S\left(V_{\mathrm{k}}, E_{k}\right), x, y\right)$ is the capacity of the maximum capacity path between nodes $x$ and $y$ in $S\left(V_{\mathrm{k}}, E_{k}\right)$ tree rooted at $x$ ).

end;

//Stage 2 is skipped

(Note: We don't execute this stage to ensure fairness as all the subgraphs $S\left(V_{k}, E_{k}\right)$ are computed using the same core topology, which results in TA for VPNs that satisfies the fairness policy defined in Section 4.2.1.) 
//Stage 3: Generate the required abstraction applying $T_{k}$

begin

//Iterate over each VPN $k \in U_{b}$ to generate the required abstraction of type $T_{k}$

Step 7: for each VPN $k$ with $T S_{k}=\left\{T_{k}, R_{k}\right\}$,

apply $l \equiv T_{k}$ on $G_{k, F M A}\left(V_{k}, E_{k}\right)$ to generate $G_{k, l}\left(V_{k}, E_{k}\right)$; (Note: $G_{k, l}\left(V_{k}, E_{k}\right)$ could be any of the four abstract topologies considered in Section 3.7.)

Step 8: flood the abstract graph $G_{k, l}\left(V_{k}, E_{k}\right)$ to the VPN CE nodes $C_{k, b}$ with periodicity $R_{k}$; end;

end;

Figure 5-4: Maximum capacity abstraction scheme for VPN topology abstraction problem with reference to Figure 4.1

\subsubsection{Mixed Bound Abstraction Scheme}

The maximum capacity abstraction algorithm may not perform well in high load conditions this is particularly true when the aggregate VPN demand request between a pair of border nodes (during the interval between two abstract topology refreshes) from multiple VPNs exceeds the capacity of the maximum capacity paths between the pair of border nodes. To address this, we propose an algorithm where, instead of a single value, we represent virtual capacity as a pair of values in the form of upper and lower bounds. Providing such bounds is expected to provide more realistic information on the available capacity in the VSP's network; it is also expected to help in handling the issue of simultaneous bandwidth requests received by a border node. This algorithm uses flow 
based algorithms in order to compute the bounds. In this scheme, the upper and lower bounds for the virtual links of the fully meshed abstraction are computed directly from the flow based algorithms without explicitly computing partition subgraphs $S\left(V_{k}, E_{k}\right)$ in Step 2 (Fig. 4.1). Therefore, the following discussion shows how the bounds for the virtual links $E_{k}$ in the fully meshed abstraction graph $G_{k, F M A}\left(V_{k}, E_{k}\right)$ are computed.

Upper Bound on Advertised Capacity: The goal of providing an upper bound on advertised capacity to a VPN with abstract topology type $l \equiv T_{k}$ is to ensure that it does not seek any more resources than the advertised upper bound during the interval between two consecutive abstract topology refreshes. For a VPN $k$ and virtual link corresponding to edge $(x, y) \in E_{k,}$, let $M_{k, i}(x, y)$ be the $i^{\text {th }}$ bandwidth request between two consecutive refresh update interval instances to be requested from the VSP, and let $\delta_{k, l}(x, y)$ be the upper bound on advertised capacity. If there are $n$ requests from a VPN $k$ in the interval $R_{k}$ then:

$$
\sum_{i=1}^{n} M_{k, i}(x, y) \leq \delta_{k, l}(x, y)
$$

We next discuss the virtual link capacity assignment for a fully meshed abstraction of a VPN hosted on border node $b$. For a VPN $k \in U_{b}$ and $x, y \in P_{k}, \delta_{k, F M A}\left(x_{2} y\right)$ is the computed upper bound capacity advertised for the virtual link connecting nodes $x$ and $y$ in a fully meshed abstraction. According to the capacity sharing policy, equal sharing implies that for all the VPNs having the common border node pair $x, y$ represented as set $Z(x, y)$, the total flow advertised must be at most $|Z(x, y)|^{*} \delta_{k, F M A}(x, y)$. Let $\alpha(x, y)$ be the 
maximum-flow ${ }^{3}$ [Ahu93] possible between border nodes $x$ and $y$, then the upper bound $\delta_{k, F M A}(x, y)$ on the advertised capacity must satisfy the following:

$$
\begin{aligned}
& |Z(x, y)|^{*} \delta_{k, F M A}(x, y) \leq \alpha(x, y) \\
& \delta_{k, F M A}(x, y) \leq\lfloor(\alpha(x, y) /|Z(x, y)|\rfloor
\end{aligned}
$$

Basically, the upper bound calculated above distributes the maximum flow from the border node $x$ to $y$ equally among all the VPNs in the set $Z(x, y)$.

Lower Bound on Advertised Capacity: In computing the lower bound $\gamma_{k, F M A}(x, y)$ for a fully meshed abstraction, the goal is to try to satisfy with a high probability any single path bandwidth request $M_{k, i}(x, y)$ during the refresh interval period $R_{k}$ from a VPN. The lower bound has to be computed carefully. A conservative lower bound estimate would lead to a VPN wrongly terminating the calls locally, while an overly optimistic estimate could lead to requests being blocked resulting in crankback of calls. Ideally, a lower bound $\gamma_{k, F M A}(x, y)$ should be set, so that the worst case simultaneous, which would be the arrival of $|Z(x, y)|^{*} \gamma_{k, F M A}(x, y)$ capacity requests, can be satisfied. Let $P_{x, y}$ be the set of edge disjoint paths between border nodes $x$ and $y$. Let $C_{x, y}$ be the minimum of bottleneck capacities of all the paths $p_{i} \in P_{x, y}$, then a lower bound can be obtained as follows:

$$
\begin{gathered}
|Z(x, y)|^{*} \gamma_{k, F M A}(x, y) \leq\left|P_{x, y}\right|^{*} C_{x, y} \\
\gamma_{k, F M A}(x, y) \leq\left\lfloor\left(\left|P_{x, y}\right|^{*} C_{x, y} /|Z(x, y)|\right)\right\rfloor
\end{gathered}
$$

\footnotetext{
${ }^{3}$ The maximum-flow between two nodes $s$ and $t$ in a graph $G(V, E)$ is total flow that can be sent from source node $s$ to sink node $t$ while preserving the capacity constraint of all the links in $E$ and conservation of flows on all nodes $V \mid(s, t)$.
} 
This approach would require computing all the edge disjoint paths between $x$ and $y$. Also, the lower bound computed using (5.5) may turn out to be very conservative because of a very poor bottleneck capacity in the set of paths $P_{x, y}$.

So, we propose to use the flow obtained from an $M$-Route flow to compute the lower bound. The concept of an $M$-Route flow was first proposed by Kishimoto [Kis92] in an effort to solve the problem of communication channel survivability in case of $(M-1)$ link failures. [Kis92] defines and proposes solutions to handle both link and node failures. Since the goal of the VPN-TA problem is to avoid contention for link resources among the VPNs hosted on a given border node, we limit our discussion to the edge disjoint version of this problem.

An edge disjoint $M$-Route flow between a source node $s$ and destination node $d$ can be defined using the definition of an elementary $M$-Route flow as follows.

An elementary M-Route flow from a node $s$ to a node $d$ is defined as a flow of one unit along each of $M$ edge disjoint paths from node $s$ to node $d$. The corresponding set of paths is called an M-path. An M-Route flow is a flow that can be expressed as a nonnegative linear sum of the elementary M-route flows.

[Kis92] also proposed an algorithm (Appendix A.3) to compute a maximum $M$-Route flow which can be found in maximum of $(M-1)$ runs of the max-flow algorithm [Ahu93] with a net complexity of $O\left(M^{*}|V|^{3}\right)$. For a given value of $M$, let the $M$-Route flow between border node $x$ and $y$ be given as $\eta(x, y)$. 


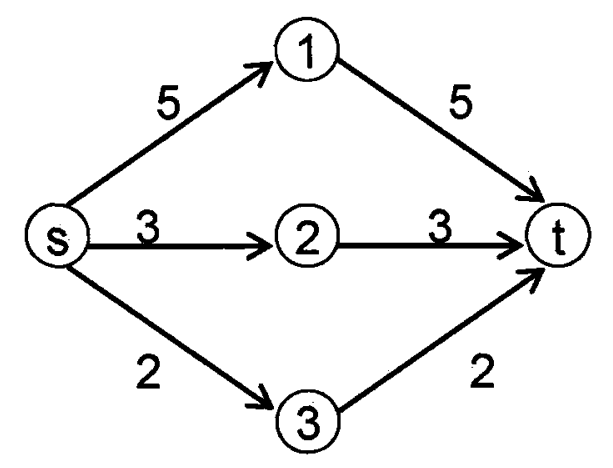

Figure 5-5: M-Route flow example

It is difficult to give a lower bound estimate that represents the single path capacity using an $M$-Route flow, since the flow represents an aggregate flow, which may not split equally among the $M$-paths as illustrated in Fig. 5.5. Here, the 2-Route flow possible from $s$ to $t$ is 10 . This is obtained by augmenting 2 unit each along paths $\{(s->1->t),(s->3->t)\}$ and 3 units each along $\{(s->1->t),(s->2->t)\}$. Hence, there are no paths between $s$ and $t$ that can handle two requests of 5 units simultaneously. So, since the split of such an $M$-Route flow is not known from the aggregate $M$-Route flow value itself, an absolute upper bound for $\gamma_{k, F M A}(x, y)$ cannot be obtained; however, we can state with high probability that $M$ simultaneously requests each value less than or equal to $\lfloor\eta(x, y) / \mathbf{M}\rfloor$ can be handled. Hence, an initial estimate of the lower bound for the virtual link in a fully meshed abstraction derived from $M$-Route flow could be set to:

$$
\gamma_{k_{F} F M A}(x, y)=\lfloor\eta(x, y) / \mathbf{M}\rfloor
$$

For an $M$-Route flow to exist, one can observe that $M$ is upper bounded by the maximum number of edge disjoint paths between $x$ and $y$. In our case, an ideal value for 
$M$ would be to assign, $M=|Z(x, y)|$, but this assignment will fail if $|Z(x, y)|$ is greater than the maximum number of disjoint paths between $x$ and $y$, which would be the case in practice. However, in general we see that the value of $M$ must satisfy, $M=\min \left(|Z(x, y)|,\left|P_{x, y}\right|\right)$. When $|Z(x, y)| \gg>\left|P_{x, y}\right|$, it will be too aggressive to use (5.6) as the lower bound estimate, hence we set the lower bound to a more practical value as in (5.7).

$$
\gamma_{k, F M A}(x, y)=\lfloor\eta(x, y) /|\mathrm{Z}(\mathrm{x}, \mathrm{y})|\rfloor
$$

The lower bound computed from $M$-Route flow in (5.7) is less conservative than the lower bound given in (5.5). We show this by comparing the lower bound values obtained by applying (5.5) and (5.7) with reference to the graph in Fig. 5.5, assuming $M=|Z(x, y)|=2$, and for border nodes $(s, t)$. Using (5.7), the lower bound $\gamma_{k, F M A}(s, t)$ is 5 units, compared to 2 units if (5.5) is used. This observation can be further generalized as:

$$
\lfloor\eta(x, y) / Z(\mathrm{x}, \mathrm{y})\rfloor \geq\left\lfloor\left(\left|P_{x, y}\right|^{*} C_{x, y} / \mathrm{Z}(\mathrm{x}, \mathrm{y})\right)\right\rfloor
$$

Assuming that all VPNs are hosted on all the border nodes; and as mentioned earlier, in practical situations we have $\forall x, y \in B \quad Z(x, y)>>\left|P_{x, y}\right|$, hence we have $M=\left|P_{x, y}\right|$ in most practical situations. Furthermore, assuming the maximum disjoint path between any two pair of border nodes $x, y \in B$ is upper bounded by $\left|P_{u}\right|$; the mixed bound algorithm complexity to determine the lower and upper bound of a fully meshed abstraction is dominated by the computation of the $M$-Route flow for each pair of border nodes. For the complexity analysis we assume the worst case maximum flow algorithm complexity of $O\left(|V|^{3}\right)$ [Ahu93]. This results in an overall complexity of $O\left(|U|^{*}|B|^{2 *}\left|P_{u}\right|^{*} \mid \eta^{3}\right)$.

Fig. 5.6 shows the pseudo code to compute the $\delta_{k, F M A}(x, y)$ and $\gamma_{k, F M A}(x, y)$ based on the above discussion corresponding to the fully meshed abstract graph $G_{k, F M A}\left(V_{k,}, E_{k}\right)$ for the 
set of VPNs $U_{b}$ hosted on a border node $b$ with reference to the TA generation process shown in Fig. 4.1. As noted earlier, in this scheme there is no explicit computation of a partition graphs $S\left(V_{\mathrm{k}}, E_{k}\right)$ for VPN $k$, so Step 2 is not executed in this case. In Step 3, we compute the lower and upper bounds for each virtual link using the maximum flow and M-Route flow values computed for each pair of border nodes for each VPN. Also, when applying this abstraction scheme, the Stage 2 in Fig. 4.1, where fairness is applied is skipped as the upper and lower bounds derived from equations (5.3) and (5.7) satisfy the faimess policy.

\section{Mixed Bound Abstraction Algorithm}

Input: $G(V, E), U_{b}, T S_{k} \forall k \in U_{b}, Z(x, y) \forall x, y \in B$.

Output: $G_{k, l}\left(V_{k}, E_{k}\right), \forall k \in U_{b}$.

begin

//Stage 1: Generate partition graphs and fully meshed abstraction begin

(Iterate the remaining steps for each VPN $k \in U_{b}$ for which abstract topology is being computed. We determine the lower and upper bound capacities of virtual links of the fully meshed abstract topology as follows).

Step 1 : identify the set of PE nodes $P_{k}$ belonging to VPN $k$;

//initialize graph $G_{k, F M A}\left(V_{k} E_{k}\right)$

for all $x, y \in V_{k}$, set $\delta_{k, F M A}(x, y)=0$ and $\gamma_{k, F M A}(x, y)=0$; (Note: For the fully meshed case the set $V_{\mathrm{k}}$ in $G_{k_{s} F M A}\left(V_{k_{0}} E_{k}\right)$ is same as $P_{k}$.) 
Step 2: (Note: In this scheme we do not compute partition subgraphs $S\left(V_{k}, E_{k}\right)$ for each VPN)

//Compute fully meshed abstraction $G_{k, F M A}\left(V_{k}, E_{k}\right)$ as follows

Step 3: for each border node $x_{2} y \in V_{k}$ in $G_{k, F M A}\left(V_{k}, E_{k}\right)$,

for each virtual link $e(x, y) \in E_{k}$, calculate max-flow $\alpha(x, y)$ between nodes $x$ and $y$; //this is the upper bound capacity set $\delta_{k, F M A}(x, y)=\lfloor(\alpha(x, y) /|Z(x, y)|\rfloor$; set $M=\min \{|Z(x, y)|$, maximum number of disjoint paths between $x$ and $y\}$; calculate $M$-Route flow $\eta(x, y)$ between nodes $x$ and $y$; //this is the lower bound capacity set $\gamma_{k, F M A}(x, y)=\lfloor\eta(x, y) /|\mathrm{Z}(\mathrm{x}, \mathrm{y})|\rfloor$;

end;

/Stage 2 is skipped

(Note: We don't include a separate step to ensure fairness; as the flows determined by applying max-flow and the $M$-Route flow algorithms between a pair of border node is equally shared with the VPNs in the set $Z(x, y)$. This result in TAs for the VPNs satisfying the fairness policy defined in Section 4.2.1.)

//Stage 3: Generate the required abstraction $G_{k, l}\left(V_{k}, E_{k}\right)$ applying $l \equiv T_{k}$ begin

//Iterate for each VPN $k$ hosted at node $b$ to generate the required abstraction Step 7: for each VPN $k$ with $T S_{k}=\left\{T_{k}, R_{k}\right\}$, apply $l \equiv T_{k}$ on $G_{k, F M A}\left(V_{k}, E_{k}\right)$ to generate $G_{k, l}\left(V_{k}, E_{k}\right)$; (Note: $G_{k, l}\left(V_{k}, E_{k}\right)$ could be any of the four abstract topologies considered in Section 3.7.) 
Step 8: flood the abstract graph $G_{k, l}\left(V_{k}, E_{k}\right)$ to the VPN CE nodes $C_{k, b}$ with periodicity $R_{k}$. end;

end;

\section{Figure 5-6: Mixed bound scheme for VPN topology abstraction problem with reference to Figure 4.1}

\subsubsection{Steiner Tree Based Abstraction Scheme}

Though the mixed bound scheme is expected to perform better than the maximum capacity abstraction scheme, the approach could perform poorly in certain situations. This may be because the maximum flow and $M$-Route flow may split the exposed capacity across multiple paths, and as a result, may not necessarily guarantee a single path in order to accommodate the capacity requested by the VPN. Other drawbacks include the complexity of the algorithm and implementation changes required in order to realize it in practice. In view of these drawbacks, we now propose a new algorithm, namely, the Steiner tree based virtual capacity computing algorithm.

Given a graph $G(V, E)$ and a subset $V^{\prime}$ of $V$, assume that each link in $E$ is associated with a cost. A Steiner tree of $G$ with respect to $V^{\prime}$ is a minimum cost tree of $G$ that contains all the nodes of $V^{\prime}$. Note that the Steiner tree may also contain some other nodes that are not in $V^{\prime}$

The goal of the proposed scheme is to build a tree for each VPN with the goal of minimally oversubscribing the residual link capacity and to use it to generate abstract topologies. The two main objectives of the tree computation process are to maximize the 
amount of capacity used for abstraction and, at the same time, minimize the capacity shared between the trees constructed for the different VPNs hosted on a border node. The objectives of the Steiner tree construction and the VPN-TA problem are correlated, since minimizing the capacity sharing of the link capacity among the VPN partition graphs will result in more accurate abstractions, which are expected to improve VPN call performance and network utilization. We achieve the first objective by computing a minimum cost Steiner tree on the graph with link costs inversely proportional to the residual capacities of the links. Since the tree needs to be computed over a subset of border nodes $P_{k}$ for a given VPN $k \in U_{b}$, a Steiner tree graph (on the border nodes of the VPN) is computed with the objective of minimizing the tree cost. In order to achieve the second objective of minimizing the capacity shared between the trees, we associate a weight with each link and increment the weight each time the link is included in the tree graph computed for a VPN.

We next discuss the abstraction algorithm, and highlight the steps required in order to compute the trees for each VPN. As in previously discussed algorithms, the trees are generated iterating over the set of VPNs $U_{b}$ sequentially. The link weight variable to measure the number of times a link has been used in the trees constructed so far is denoted as $w(e)$. Initially, this link variable is set equal to a value of 1 . Another cost variable $c(e)$, used to compute the Steiner tree is initialized as a function of $w(e)$ and the link residual capacity $r(e)$. We set $c(e)$ to $w(e) *(t(e)) /(r(e))$, here $t(e)$ is the total capacity of the link $e$. The reason for such initialization is to ensure that the links with the more residual capacity would have a lower cost in comparison to links with higher residual 
capacity. We next compute the Steiner tree, $S\left(V_{k}, E_{k}\right)$, for a VPN $k$, with edge cost initialized to $c(e)$. For each VPN, Steiner trees are computed with respect to all the border nodes in the set $P_{k}$ as the root node. After the subgraphs are computed for each of the VPNs, we set the capacity of the virtual link $(x, y) \in E_{k}$ of the fully meshed abstract graph to the bottleneck capacity of the path connecting the root node $x$ to the border node $y$ in the Steiner tree $S\left(V_{k} E_{k}\right)$. Before continuing with the computation of subgraphs for the next VPN, the weight of the link variable $w(e)$ corresponding to $e \in E_{k}$ in $S\left(V_{k}, E_{k}\right)$ is incremented by 1 . This increment dissuades the future subgraph computations from using the links that are part of the already previously computed VPN subgraphs; it also enables links with lesser cost, i.e. more residual capacity and least used graph edges, to be considered during the tree computation.

Since the trees are computed in sequence, the virtual capacities computed for each VPN corresponding to the fully meshed abstract graphs are going to be different. Hence, in this scheme, the fairness criterion is applied by executing Steps 4-6 in Fig. 4.1. The Steiner tree computation is a strongly NP-complete problem. Literature provides good heuristics to compute Steiner trees. We use a simple directed Steiner tree algorithm from [Kod00] (Appendix A.4) for tree computation whose complexity to compute a Steiner tree rooted at a border node to the remaining border nodes (assuming $P_{k} \equiv B$ ) is $O\left(|B|^{*}(|E|+|V| \log (|V|))\right)$. The complexity of deriving a fully meshed abstract topology for a given VPN set $U$ is $O\left(|U|^{*}|B|^{*}\left(|B|^{*}(|E|+|V| \log (|V|))\right)\right)$. 
Fig. 5.7 is the pseudo code of an algorithm for generating partition graphs and abstractions from them using the Steiner tree approach with respect to the TA generation process shown in Fig. 4.1.

\section{Steiner Tree Topology Abstraction Algorithm}

Input: $G(V, E), U_{b}, T S_{k} \forall k \in U_{b}$.

Output: $G_{k, l}\left(V_{k}, E_{k}\right), \forall k \in U_{b}$.

begin

//Stage 1: Generate partition graphs and fully meshed abstraction

begin

//Initialize edge weight and cost

for all edge $e \in E$ in $G(V, E)$,

$$
\begin{aligned}
& \text { set } w(e)=1 ; \\
& \text { set } c(e)=w(e)^{*}(t(e)) /(r(e)) ;
\end{aligned}
$$

(Iterate the remaining steps for each VPN $k \in U_{b}$ for which abstract topology is being computed. We determine the capacities of virtual links of the fully meshed abstract topology as follows).

Step1: identify the set of PE nodes $P_{k}$ belonging to VPN $k$;

//initialize graph $G_{k, F M A}\left(V_{k}, E_{k}\right)$

for all $x, y \in V_{k}$, set $w_{k F M A}(x, y)=0$; (Note: For the fully meshed case the set $V_{\mathrm{k}}$ in $G_{k, F M A}\left(V_{k}, E_{k}\right)$ is same as $\left.P_{k}\right)$ 
Step 2: compute the Steiner tree $S\left(V_{k}, E_{k}\right)$ rooted at each border node $x \in P_{k}$, spanning all the nodes in $y \in P_{k}$ and $y \neq x$;

//Generate $G_{k, F M A}\left(V_{k}, E_{k}\right)$ using $S\left(V_{k}, E_{k}\right)$ rooted at each $x \in P_{k}$

Step 3:

for each $(x, y) \in E_{k}$ in $G_{k, F M A}\left(V_{k}, E_{k}\right)$,

let $P=\left\{x, e_{l}, \ldots e_{k}, y\right\}, e_{i} \in S\left(V_{k}, E_{k}\right)$; (Note: $P$ is the path from $x$ to $y$ in the Steiner tree rooted at $x$ );

set $w_{k . F M A}(x, y)=\min \left\{r\left(e_{1}\right), \ldots, r\left(e_{k}\right)\right\}$; (Note: $w_{k . F M A}(x, y)$ is the bottleneck capacity path between nodes $x$ and $y$ in $S\left(V_{\mathrm{k}}, E_{k}\right)$ )

//increment the link weights used in the subgraphs $S\left(V_{\mathrm{k}}, E_{k}\right)$ rooted on $P_{k}$ for each border node $x \in P_{k}$,

for each Steiner tree $S\left(V_{\mathrm{k}}, E_{k}\right)$ rooted $x$, for each edge $e \in E_{k}$ in $S\left(V_{k}, E_{k}\right)$ and $e \in G(V, E)$,

$$
\text { set } w(e)=w(e)+1 ; \text { set } c(e)=w(e)^{*}(t(e)) /(r(e)) ;
$$

end;

/Stage 2: Apply fairness criteria to the VPN set $U_{b}$ as in Fig. 4.1

begin

Steps 4-6: apply faimess policy to the set of fully meshed abstractions $G_{k, F M A}\left(V_{k}, E_{k}\right)$, $\forall k \in U_{b}$;

end;

//Stage 3: Generate the required abstraction applying $T_{k}$

begin

(Iterate over each VPN $k \in U_{b}$ to generate the required abstraction of type $T_{k}$ )

Step 7: for each VPN $k$ with $T S_{k}=\left\{T_{k}, R_{k}\right\}$, apply $l \equiv T_{k}$ on $G_{k, F M A}\left(V_{k}, E_{k}\right)$ to generate $G_{k, l}\left(V_{k}, E_{k}\right)$; (Note: $G_{k, l}\left(V_{k}, E_{k}\right)$ could be any of the four abstract topologies considered in Section 3.7.) 
Step 8: flood the abstract graph $G_{k, l}\left(V_{k}, E_{k}\right)$ to the VPN CE nodes $C_{k, b}$ with periodicity $R_{k}$; end;

end

Figure 5-7: Steiner tree based scheme for VPN topology abstraction problem with reference to Figure 4.1

\subsubsection{Use of Virtual Link Capacity for Route Computation}

In the context of the TA service, the virtual link capacity associated with the TA is used by the CE node to check for the availability of the path in the VSP's core network with respect to the requested capacity (feasibility check). Based on the outcome of the path computation and successful signaling of the path to the destination CE node, the servicing PE node sends a positive or negative acknowledgement to the CE node initiating the path request. Next, we comment on the usage of the virtual link capacity metric during the path computation by the CE node.

The value of a single metric (as in the maximum capacity abstraction or the Steiner tree abstraction scheme) or the lower bound in the mixed bound abstraction scheme represents the maximum capacity value of any bandwidth request that can be required from the VSP during the duration defined by $R_{k}$. If the requested capacity does not exceed this value, then the $\mathrm{CE}$ will determine that the request is feasible.

The upper bound metric in the mixed bound abstraction scheme indicates the total capacity that can be requested during an abstract topology refresh interval. Each time a 
virtual link appears in a feasible path, the requested capacity should be subtracted from the upper bound of the virtual link. Hence, at any point in time, the upper bound will reflect the amount of capacity that can still be requested from the VSP within the interval defined by $R_{k}$.

In order to enable the use of the upper and lower bound values in a practical network, changes are required to the path computation algorithm executed in the VPN CE router. Routing protocols like OSPF employ the constrained Dijkstra's algorithm to compute bandwidth constrained paths. The constrained Dijkstra's algorithm has two stages; in the first stage, the links that do not satisfy the requested bandwidth are pruned, and in the second stage, the shortest path algorithm to compute the path is applied. The use of bounds provided using the mixed bound scheme in the route computing algorithm can be incorporated during the stage where links that do not satisfy the bandwidth constraints (lower and upper bounds) are pruned. If the path computation is successful (indicating that a feasible path is available), the path request is signalled to the PE node. Upon successful signalling, the upper bound capacity is updated by subtracting the successfully requested capacity from the virtual links of the associated abstract topology.

\subsection{Simulation and Performance Evaluation}

In this section, we discuss the simulation results, which compare the performance of the three TA generation schemes described in the previous section. These schemes are studied in the context of the MDVF discussed in the previous chapter. 


\subsubsection{Performance Metrics}

The performance metrics are the same as those introduced in Section 4.3 of Chapter 4, which are success ratio, crankback ratio, misscall ratio, and network utilization.

\subsubsection{Simulation Setup}

The simulation setup and the network topologies used for the analysis are the same as those discussed in Section 4.3.2. The results discussed in the section have been obtained from 30 independent replications to achieve an absolute error of less than $1 \%$ and C.I of $95 \%$. Table 5.8 shows the simulation parameters used in our simulation scenarios for evaluating the abstraction schemes. The load in the simulation is varied in two ways. In the first case, mean call holding time, $H$, is varied between $[10,1000] \mathrm{s}$; here, the VPN requested bandwidth is uniformly distributed between $[1,500]$ units. In the second case, the performance of the schemes is evaluated by varying the upper bound of the uniformly distributed requested capacity, $X$, in the range of $[100,1000]$ units, in this case the mean inter-arrival time and mean holding time are set to $100 \mathrm{~s}$. As this simulation scenario focuses on studying the performance of the abstraction schemes, the choice of which type of abstract topology for the VPNs should be such that its granularity minimizes its effect on the conclusions made on the performance of the abstraction schemes. Like the fully meshed abstraction, the source-star abstraction is unambiguous in terms of connectivity and is non-lossy with respect to bandwidth metric, with the added advantage of low overhead. Hence, all the VPNs in this scenario are provided with source-star abstraction. Though the results presented next may vary with different choice of abstract topology 
type parameter $T_{k}$, the difference in terms of the performance metrics should be the same as that studied as part of objectives $1 \& 2$ in Section 4.3 .2 in Chapter 4.

\begin{tabular}{|l|c|}
\hline \multicolumn{1}{|c|}{ Simulation Parameters } & Values \\
\hline VPN Mean Call Inter-arrival Time (s) (Exponential Dist.) & 100 \\
\hline VPN Mean Call Holding Time (s) (Exponential Dist.) & {$[10,1000]$} \\
\hline VPN Bandwidth Request (Uniform Distribution) & {$[1,500]$} \\
\hline Core Topology Update Interval (s) & 5 \\
\hline VPN Abstract Topology Type (VPN (A-E)) & SSA \\
\hline Abstract Topology Refresh Interval (VPNA) (s) & 100 \\
\hline Abstract Topology Refresh Interval (VPNB) (s) & 100 \\
\hline Abstract Topology Refresh Interval (VPNC) (s) & 100 \\
\hline Abstract Topology Refresh Interval (VPND) (s) & 100 \\
\hline Abstract Topology Refresh Interval (VPNE) (s) & 100 \\
\hline
\end{tabular}

Table 5.1: Simulation parameters to evaluate decentralized abstraction schemes

Figs. 5.8-5.15 compare the success, crankback and misscall ratios, and the network utilization for each of the three abstraction algorithms with increasing load $(H)$ and varying VPN capacity request requirements $(X)$ for Network-1. Figs. 5.16-5.17 shows the results for Network-2 and Figs. 5.18-5.19 for Network-3. Because of the similarity of the results for the three topologies, we first discuss in detail the results for Network-1, and then summarize the performance for Network-2 and Network-3. 


\subsubsection{Comparison of Success Ratio}

Fig. 5.8 compares the success ratio of the three schemes. Here, the load is varied by increasing the mean call holding time. Increasing the call holding times causes calls to remain in the network for a longer period of time, and as a result, the average link residual capacity reduces with time. This results in an increasing variance of link capacity across the network. Hence, with an increasing load on the network, the TA information with the VPNs is expected to be more asynchronous with the physical state of the network. This causes the average call performance to decrease, which is the trend observed in Figs. 5.8-5.9.

Of the three schemes we observed that, the maximum capacity scheme performs the best. Its success ratio decreases by about $15 \%$ as the load is increased from 0.1 to 10 Erlangs. The next best scheme is the Steiner tree abstraction scheme whose success ratio performs as well as the maximum capacity scheme at over the range of load conditions; on the whole even its performance decreases by about $15 \%$. The poorest performance is displayed by the mixed bound scheme, whose performance decreases by about $48 \%$ at the maximum load. The poorest performance of the mixed bound scheme is because of its conservative mode of exposing capacity which divides the available maximum flow and $M$-Route flow among all the VPNs equally to facilitate the PE node to handle worst case capacity requests from the VPNs simultaneously. This result in the CE nodes terminating calls locally resulting in poor miscall ratio performance which we discuss later. The best success ratio performance of the maximum capacity scheme indicates the usefulness of being aggressive in a dynamic bandwidth request scenario enabled by the TA service. 
A similar difference in performance among the abstraction schemes is also noted when bandwidth request size $(X)$ is varied. This is shown in Fig. 5.9. The key difference here is that the rate of decrease of success ratio performance is higher compared to the previous case. This is expected due to the following reason. With the increasing value of $X$, the average size of the VPN bandwidth request increases. After a certain point, the average request size is greater than the virtual link bandwidth associated with the TA. This causes more calls to be rejected locally in the CE node, which subsequently leads to higher miscall ratio. This results in a steep deterioration of the VPN call performance, as observed in Fig. 5.9. For the maximum capacity scheme, the performance decreases by $25 \%$ at a maximum bandwidth request of 1000 units. For the Steiner tree scheme, this decline of performance is $27 \%$, while for the mixed bound scheme, the percentage decrease is $46 \%$. The reason for this difference in performance between the mixed bound scheme and the other two are the same as stated earlier with respect to Fig. 5.8. Steiner tree performs only $1 \%-3 \%$ poorer when compared to the maximum capacity scheme due to its less aggressive nature. This performance difference again points to the fact that, as the mean bandwidth request size increases, an aggressive scheme, such as the maximum capacity scheme, which tends to expose more resources, results in better successful route computation locally at the CE node in comparison to the other two schemes. 


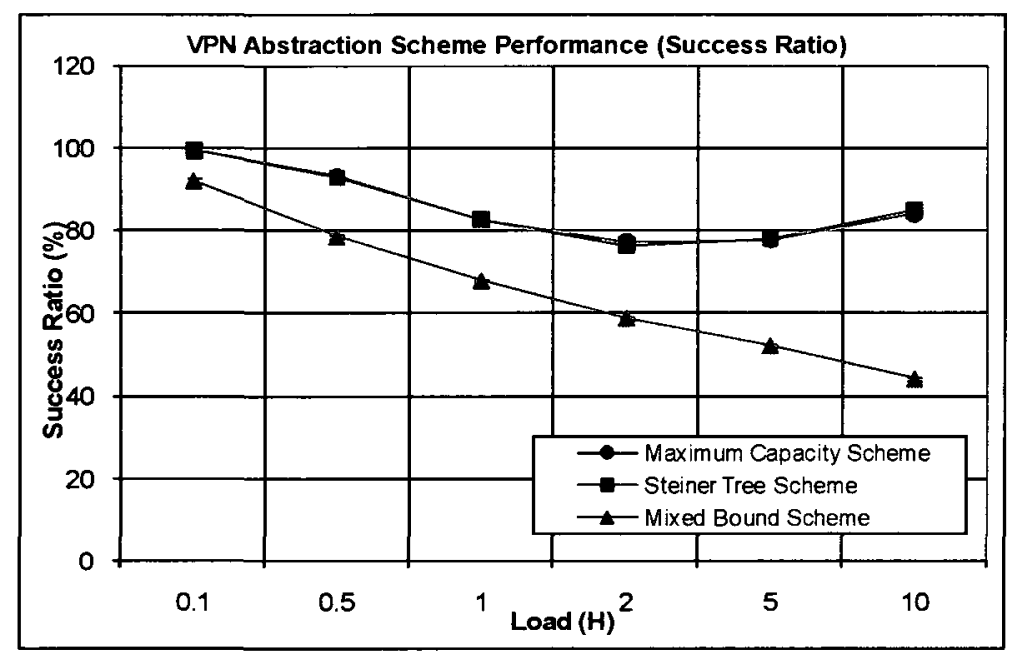

Figure 5-8: VPN call performance comparing VPN success ratio with varying mean holding time (H) with 95\% C.I (Network-1)

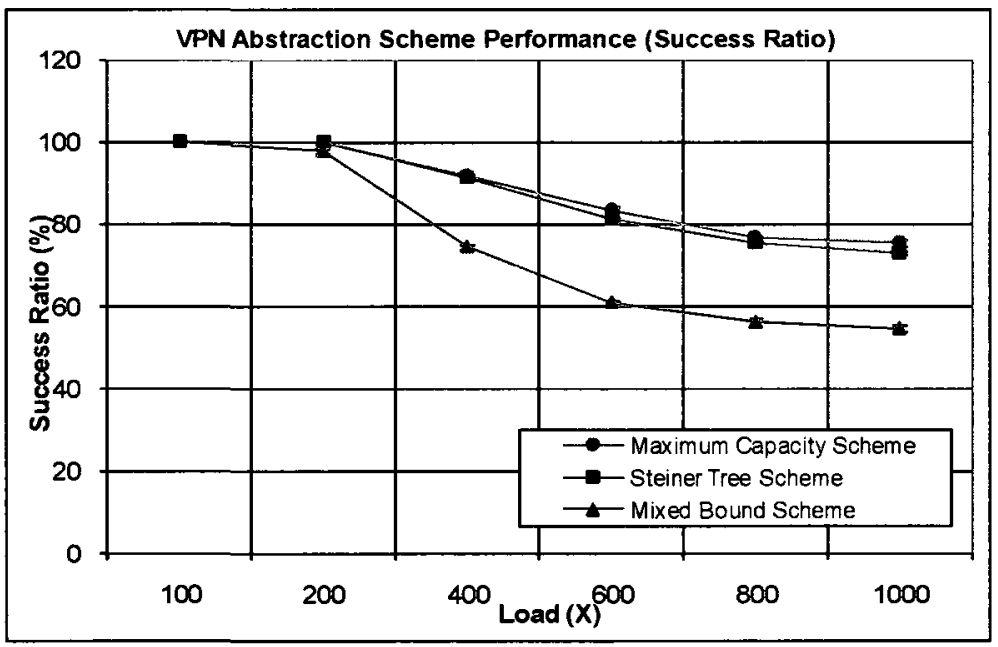

Figure 5-9: VPN call performance comparing VPN success ratio with varying bandwidth request $(X)$ with $95 \%$ C.I (Network-1)

\subsubsection{Comparison of Crankback Ratio}

Fig. 5.10 compares the crankback ratio for the three schemes with increasing $H$. The VPN call performance for all the three schemes deteriorates with an increasing load on the network. This can be attributed to two reasons: the first reason is the oversubscription, 
which is still an issue for the three schemes, and the second reason is that the increasing load causes a reduction in the correctness of the virtual capacity information associated with the abstraction with respect to the core state of the network. This causes calls to be successfully computed by the CE node locally but cranked back by the PE node due to a lack of resources in the core.

Comparing the performance of the abstraction schemes, we note that the maximum capacity scheme performs poorer than the other two schemes, which illustrates one of the drawbacks of employing an aggressive scheme; despite the fact that it performs the best in terms of success ratio metric. The mixed bound scheme performs the best with respect to this metric; its crankback ratio was noted to be less than $2 \%$, while the same was noted to increase by $13 \%$ over the range of load conditions for maximum capacity scheme. The good performance of mixed bound scheme can be attributed to its conservative approach of assigning lower and upper bounds to the virtual links using the maximum flow and $M$-Route flow approaches. Another reason why mixed bound scheme has such a low crankback ratio is also because of large number of calls being terminated locally at the CE nodes, resulting in high miscall ratio as we see next. The performance of the Steiner tree scheme lies between the performances of the other two schemes, with its performance being $1 \%-3 \%$ better than the maximum capacity scheme.

With reference to Fig. 5.11, which compares the crankback ratio with the increasing value of $X$, we note similar observations. Here, we again observe the advantage of applying mixed bound scheme in comparison to the other two schemes if the objective is to minimize the crankback ratio. The crankback ratio at the maximum 
load condition is observed to be less than $2 \%$ for mixed bound scheme, compared to $14 \%$ for maximum capacity scheme and $11 \%$ for Steiner tree scheme.

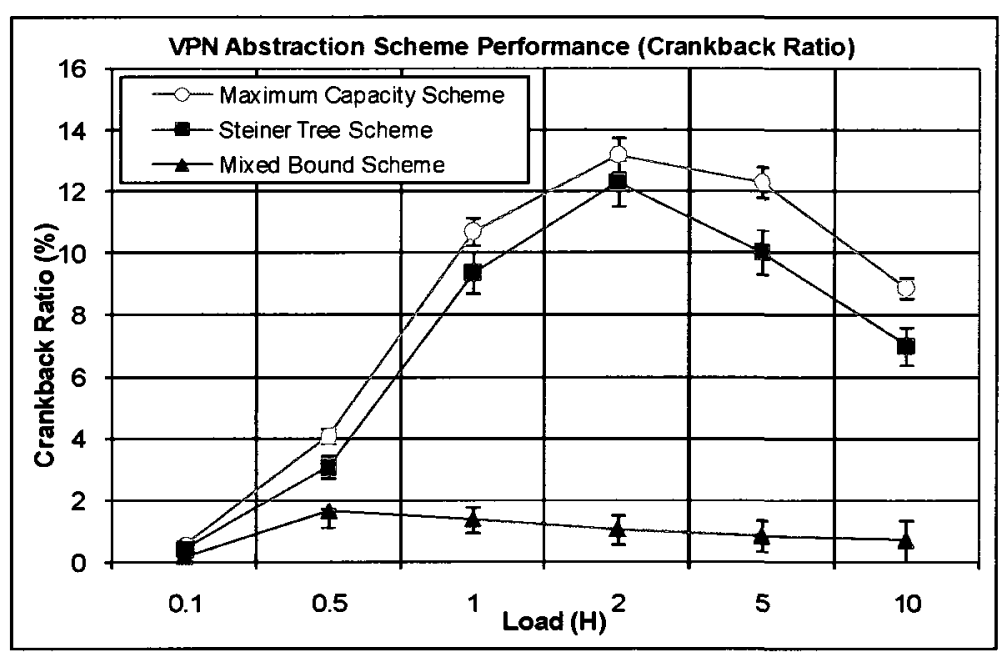

Figure 5-10: VPN call performance comparing VPN crankback ratio with varying mean holding time (H) with 95\% C.I (Network-1)

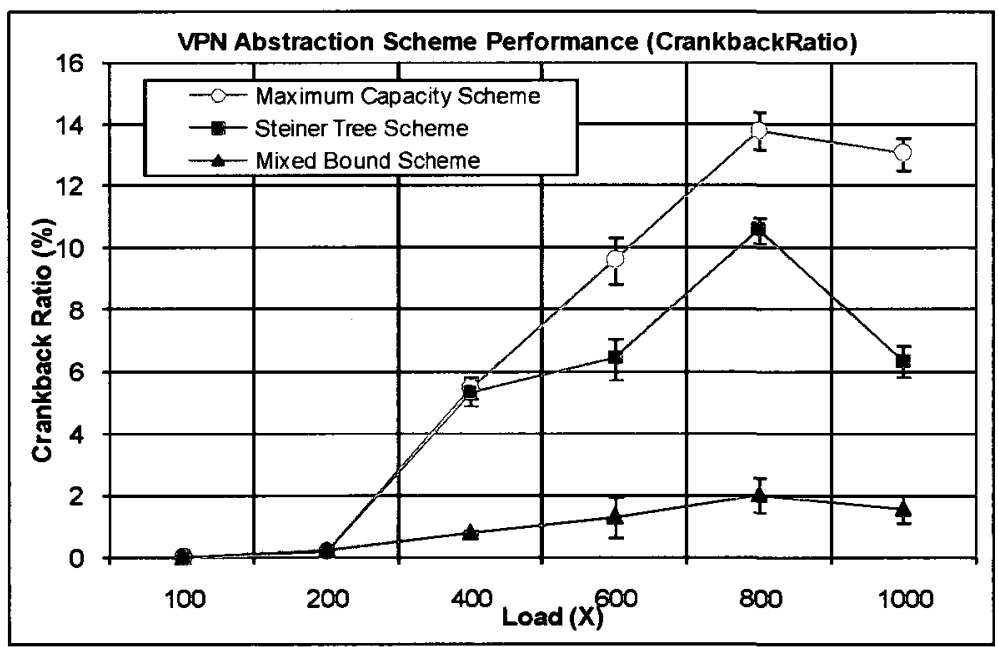

Figure 5-11: VPN call performance comparing VPN crankback ratio with varying bandwidth request (X) with 95\% C.I (Network-1) 


\subsubsection{Comparison of Misscall Ratio}

With respect to miscall ratio, in Fig. 5.12, the maximum capacity scheme displays the best performance of the three algorithms. Again, this can be attributed to its aggressive nature, where the higher values of exposed virtual link capacities allow the VPNs to successfully compute paths without locally rejecting them, which results in better misscall ratio performance even at high load conditions. However, this also leads to poor performance with respect to the crankback ratio, as seen previously. The next best performance is displayed by the Steiner tree abstraction scheme, which performs $1 \%-2 \%$ poorly when compared to maximum capacity scheme, but gains in terms of crankback ratio. The mixed bound algorithm displays the poorest performance of the three abstraction schemes. Its performance is observed to deteriorate by $48 \%$ over the range of load conditions; the reason being that a lot of calls were rejected locally when the capacity demands of the VPNs exceeded the conservative lower bound of the virtual link capacity associated with the TA.

Fig. 5.13 compares the performance of the schemes when the maximum bandwidth request from the VPN is varied. The performance displayed by the three schemes and the conclusions are similar to those discussed in the previous case. We observe that the maximum capacity scheme performs the best in terms of this metric, but $4 \%$ poorer than the previous case. This correlates with the lesser success ratio achieved as observed in the context of success ratio performance. At the maximum load, the maximum capacity scheme achieves a $11 \%$ misscall ratio, in comparison to $13 \%$ when Steiner tree scheme is applied, and $44 \%$ in the case of the mixed bound scheme. 


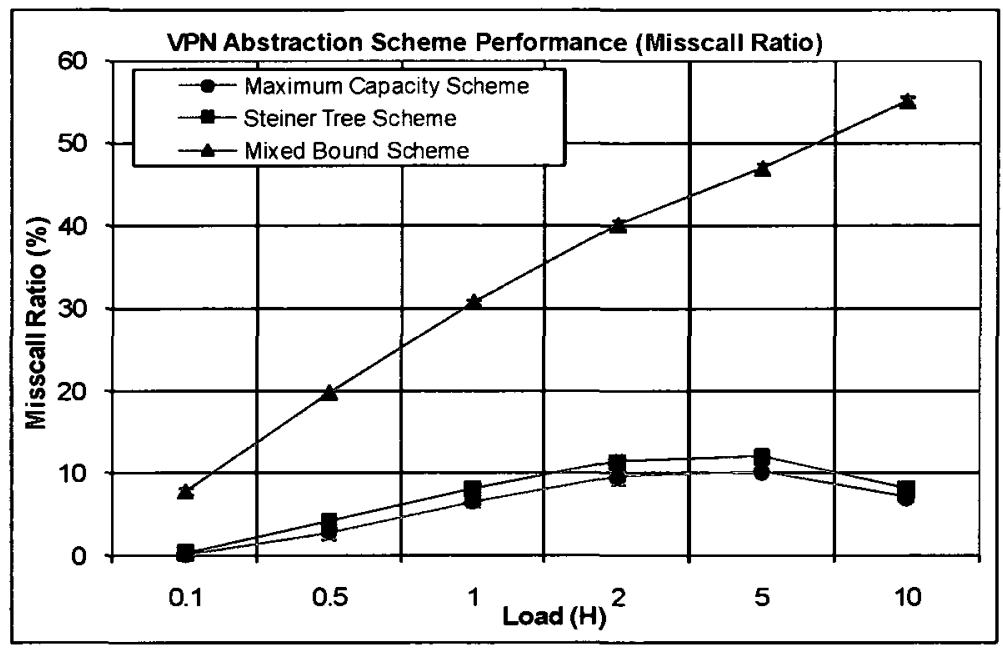

Figure 5-12: VPN call performance comparing VPN misscall ratio with varying mean holding time (H) with $95 \%$ C.I (Network-1)

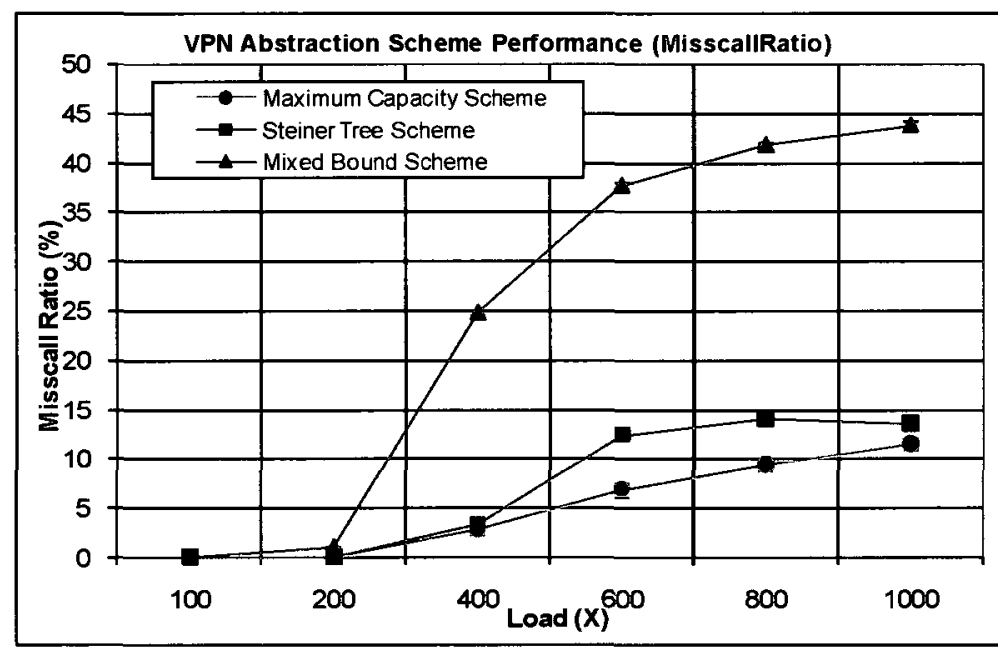

Figure 5-13: VPN call performance comparing VPN misscall ratio with varying bandwidth request $(X)$ with $95 \%$ C.I (Network-1)

\subsubsection{Comparison of Core Network Utilization}

Fig. 5.14 compares the effect of abstraction schemes on the core network utilization metric. It is also important to note that although network utilization in the context of the 
TA service is influenced by the abstraction schemes, it also depends on the optimizing principles used by the core routing algorithm, which in this case is the constrained Dijkstra's algorithm. From Fig. 5.14, we observe that the maximum capacity abstraction scheme results in better network utilization than the other two abstraction schemes. The higher network utilization for the maximum capacity scheme can be attributed to its aggressive nature in sharing resources, which also results in the best performance in terms of success ratio (Fig. 5.8). The performance of the Steiner tree scheme is slightly poorer by $3 \%$ in comparison to that of the maximum capacity scheme, which is also inline with the poorer performance in terms of misscall ratio observed in Fig. 5.12. Of the three schemes, the mixed bound scheme shows the poorest network utilization performance due to its high miscall ratio (Fig. 5.12) resulting from its conservative capacity exposing strategy.

Fig. 5.15 compares the network utilization achieved when the upper bound of the uniformly distributed requested capacity $X$ is increased. As expected, an increase in an upper bound of requested capacity results in an increase in network utilization, but an important observation here is the decreasing trend in the graph in the cases of the three schemes when $X>400$. This is because, when $X<400$, i.e. during low load condition the crankback ratio (Fig. 5.11) increases at a faster rate compared to the misscall ratio (Fig. 5.13) for all the three schemes, as a result new calls are admitted into the network and the network utilization increases. When $X>400$, an increasing number of calls are locally rejected by the $\mathrm{CE}$ node, which results in the increase in the rate of miscall ratio (Fig. 5.13) compared to the crankback ratio (Fig. 5.11), due to which fewer path requests get 
past the VPNs to the VSP, as a result the network utilization begins to flatten and decrease.

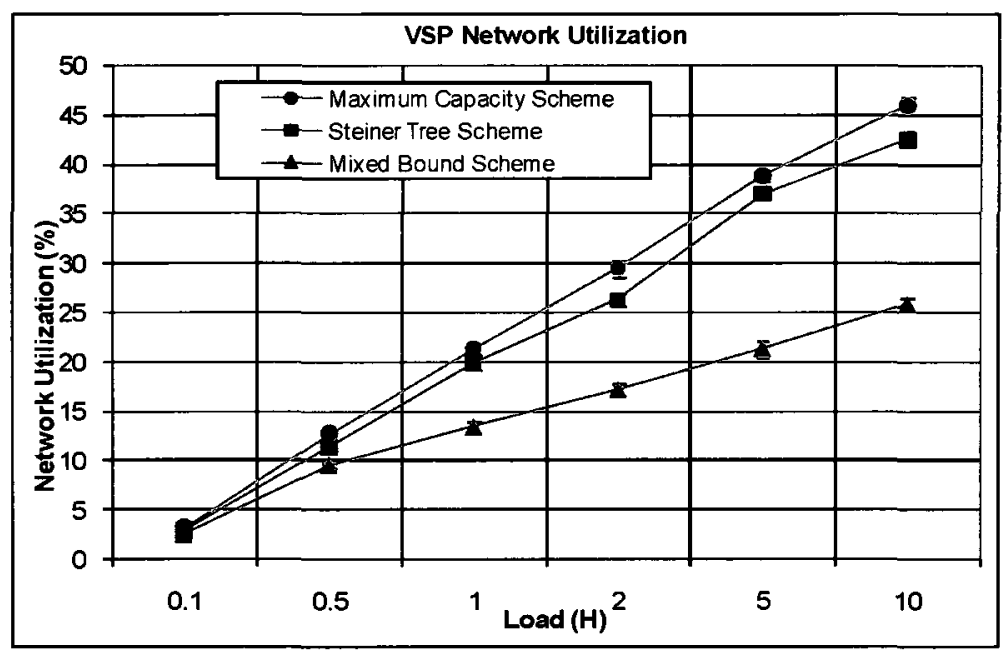

Figure 5-14: Comparing VSP core network utilization with varying mean holding time (H) with $95 \%$ C.I (Network-1)

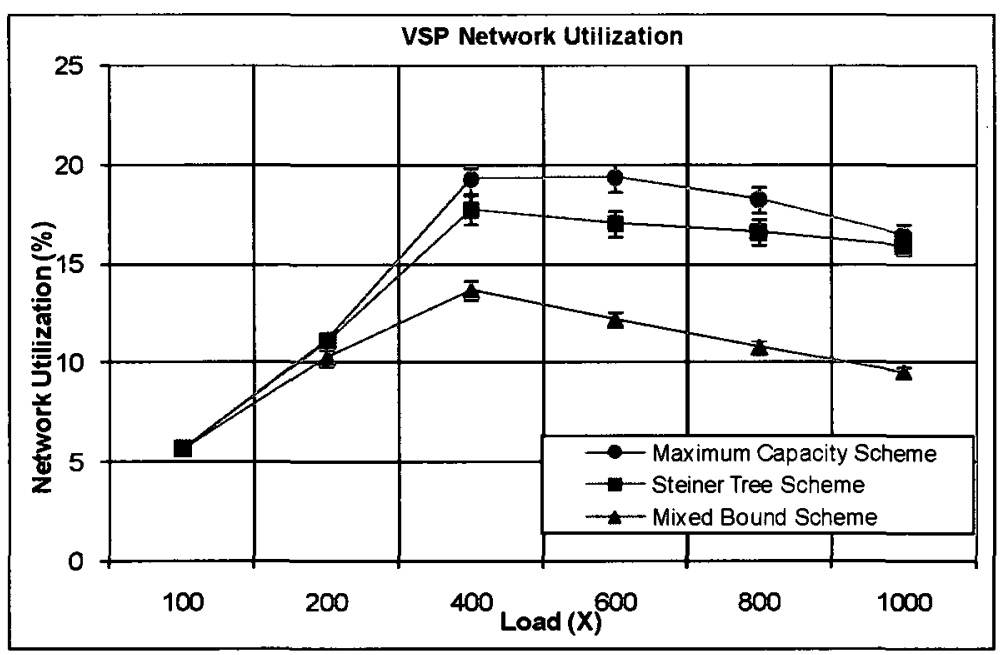

Figure 5-15: Comparing VSP core network utilization with varying bandwidth request (X) with $95 \%$ C.I (Network-1) 


\subsubsection{Performance for Network-2 and Network-3}

Figs. 5.16-5.17 compare the performance of the abstraction schemes in terms of call performance and network utilization for Network-2. Figs. 5.18-5.19 compares the performance for Network-3.

For the case of Network-2, the important observation to be made is the reduced difference in performance between the mixed bound scheme and the other two schemes compared to what was observed in the case of Network-1, i.e., the conservative nature of the mixed bound scheme becomes less of a dominating factor with a network of smaller size; the reason for this can be attributed to the overall lesser network capacity and hence lesser achievable maximum flow between any pair of border nodes, which results in very good misscall ratio performance of $20 \%$, compared to $55 \%$ for Network-1. For the mixed bound scheme, we also observe that, the crankback ratio is in the range of $7 \%$, which is $5 \%$ higher than in the case of Network- 1 . This is because of its aggressive nature as discussed earlier which correlates with the high success ratio achieved by the mixed bound scheme. We also note that the Steiner tree scheme performs $1 \%-2 \%$ better than maximum capacity scheme. This shows that being too aggressive as in the case of maximum capacity scheme with smaller networks could lead to poor call performance.

The performance for Network-2 was noted to be similar to Network-1. In this case the maximum capacity scheme outperforms Steiner tree scheme by $1 \%-2 \%$. Similar gain in performance was also noted in terms of misscall ratio and network utilization metrics. In terms of crankback ratio Steiner tree performed better than the maximum capacity scheme. Mixed bound scheme performed the best in terms of crankback ratio 
performance, but significantly poorer compared to maximum capacity scheme and Steiner tree scheme in terms of success and network utilization metrics.
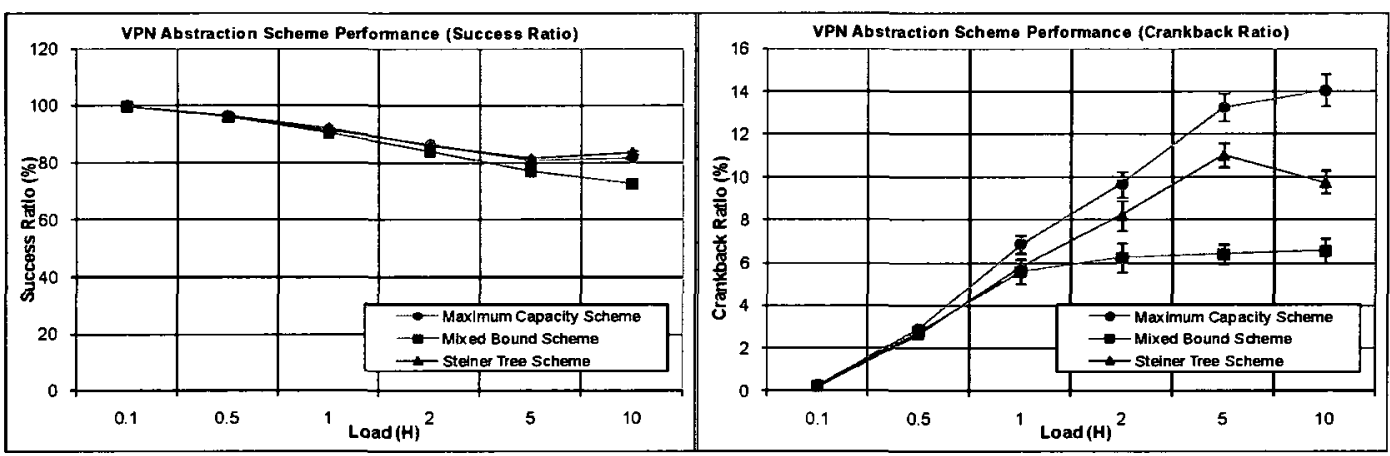

Figure 5-16(a-b): Success and crankback ratio with varying mean holding time (H) with $95 \%$ C.I (Network-2)
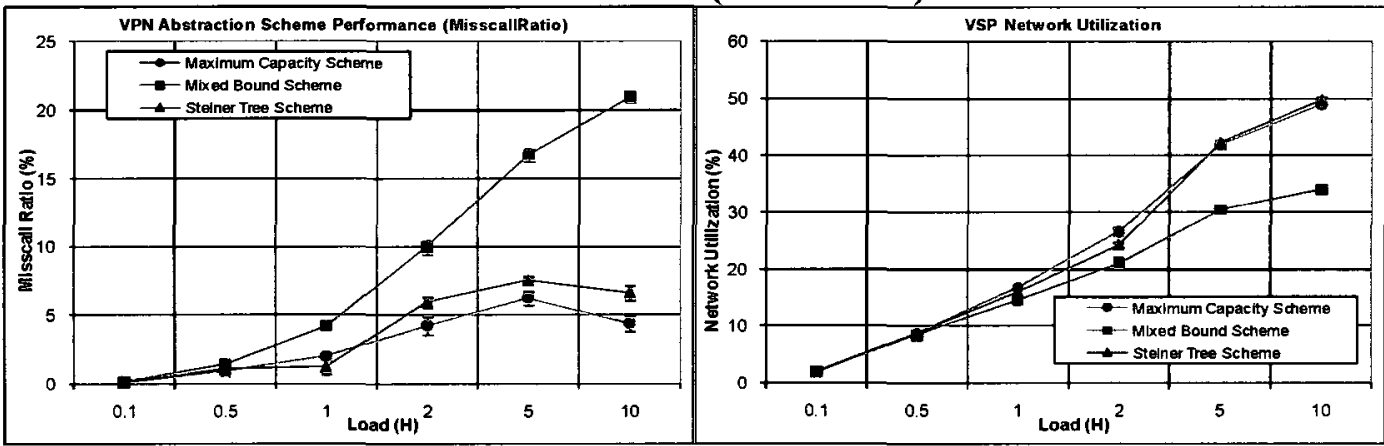

Figure 5-17(a-b): Miscall ratio and network utilization with varying mean holding with 95\% C.I time (H) (Network-2)
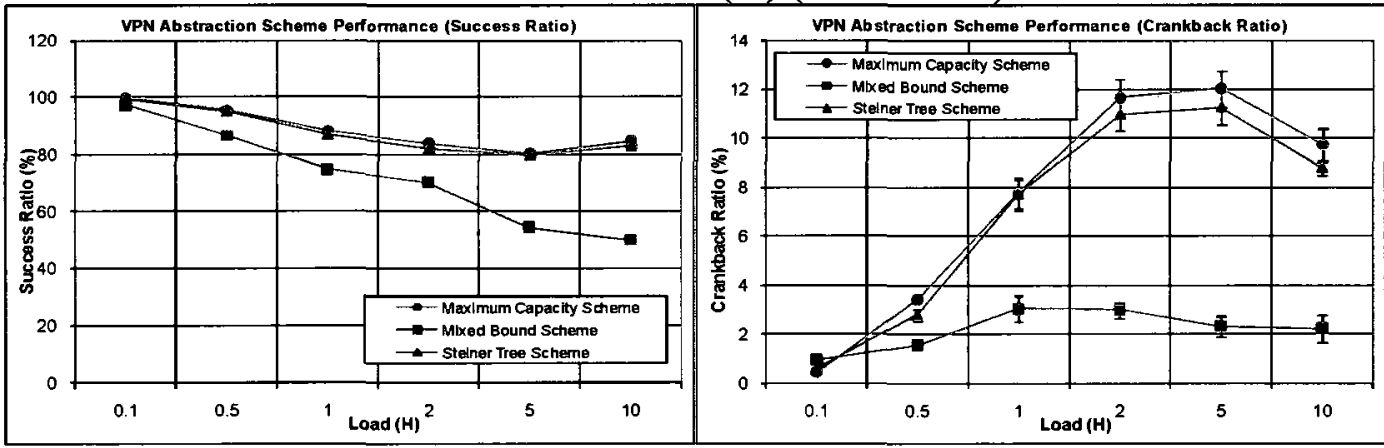

Figure 5-18(a-b): Success and crankback ratio with varying mean holding time (H) with $95 \%$ C.I (Network-3) 

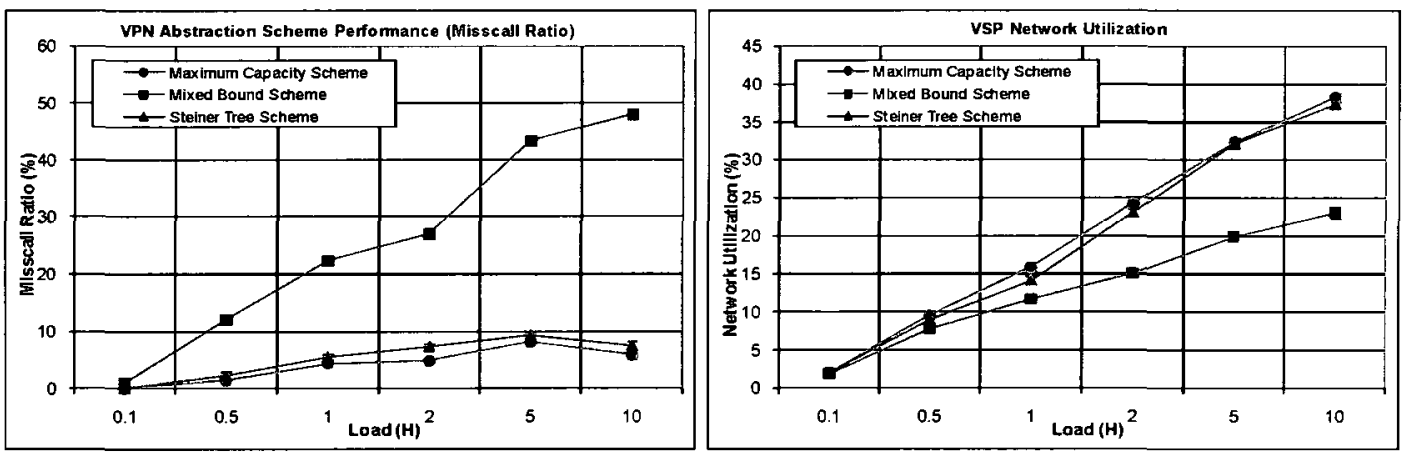

Figure 5-19(a-b): Miscall ratio and network utilization with varying mean holding time (H) with 95\% C.I (Network-3)

\subsubsection{Summary of Performances of the Topology Abstraction Schemes}

From the simulation results, we observe that the three abstraction schemes display different properties. Overall, the maximum capacity scheme performs the best among the three abstraction schemes. This shows that the aggressive approach of exposing capacity using the maximum capacity algorithm does prove to be effective in a dynamic service scenario. However, it should also be mentioned that the aggressive nature of the maximum capacity scheme also results in a higher call crankback ratio in comparison to the other schemes. In regards to the miscall ratio, the maximum capacity scheme displays the best performance of the three algorithms. Again, this can be attributed to its aggressive nature, where the higher values of the exposed virtual link capacities allow the VPNs to successfully compute paths without locally rejecting them, and this subsequently results in a better misscall ratio performance but affecting its crankback ratio performance as the load increases. 
With respect to mixed bound scheme we saw that it performed the best with respect to crankback ratio metric, which suggests that being conservative helps reduce call crankbacks but at the cost of poor success, miscall ratio, and network utilization metrics.

With respect to the Steiner tree scheme, we see that this abstraction scheme outperforms the mixed bound scheme in terms of success ratio, and its performance is same as or only slightly poorer in comparison to the maximum capacity scheme. This can be attributed to the nature of the abstraction scheme, where the abstract topology is derived from minimally overlapping trees, which causes it to be less aggressive than maximum capacity scheme and less conservative than the mixed bound scheme. This underscores the fact that the Steiner tree scheme strikes a good balance in terms of all three call performance metrics.

\subsection{Summary}

Our main objective in this chapter was to develop algorithms in order to generate topology abstractions in the decentralized manner in the context of the TA service to address the issue of oversubscription. In this context, we defined the VPN topology abstraction (VPN-TA) problem with the goal of maximizing the VPN success ratio and network utilization. As a way of solving this problem, we proposed three abstraction schemes: maximum capacity, mixed bound, and Steiner tree scheme.

From our study of the performance of the abstraction schemes, we conclude that the abstraction schemes demonstrated a trade-off between the call performance metrics 
and network utilization. A VSP's choice of the abstraction scheme for decentralized TA generation will ultimately depend on its service objectives. If the goal is to maximize the network utilization and success ratio, the maximum capacity algorithm proves to be the best. However, if the goal is to minimize the crankback performance for the VPNs, the mixed bound algorithm proves to be the best. Finally, we saw that the Steiner tree approach performed as well as the maximum capacity scheme in terms of the success ratio, network utilization, and second best in terms of crankback ratio metric; this makes it a good abstraction scheme if the objective is to strike a balance between the performance metrics. 


\section{Chapter 6}

\section{Centralized VPN Topology Abstraction}

\subsection{Introduction}

As pointed out in the previous chapter, the drawbacks of the decentralized approach are issues related to oversubscription of available resources and the possibility of border nodes being required to serve multiple VPN requests during high load conditions in the context of the TA service to the VPNs. We tried to address these issues partially by using three TA schemes that differed in terms of call performance and network utilization. Another approach used to address the issue of oversubscription is to generate the TAs in a centralized manner; this is the topic explored in this chapter.

In Section 6.2, we discuss the process of TA generation derived from VPN subgraph partitions generated in a centralized manner. Section 6.3 defines a problem that arises in the context of the centralized mode of TA generation called the VPN core capacity sharing (VPN-CS) problem. We investigate this problem using new algorithms based on the multicommodity flow theory. In particular, we analyze the applicability of the maximum concurrent flow (MConF) theory and maximum multicommodity flow 
(MMCF) theory in terms of their abstraction efficiency and fairness; we use these formulations to propose new online resource partitioning schemes in order to generate TAs with the objective of maximizing VPN call performance and core network utilization. Furthermore, in order to address the fairness issue associated with the MMCF based partitioning scheme, Section 6.4 discusses variations to the MMCF formulation in order to improve fairness in the case of an offline implementation. We also propose a new fair balancing algorithm for online implementation. In Section 6.5, the performance of these scalable resource partitioning schemes and the proposed fairness enhancements are studied using offline analysis as well as simulation of the MDVF in which the VPN TAs for the TA service are generated in a centralized manner.

\subsection{Centralized Topology Abstraction Process}

The main difference between the centralized form of TA generation and the TA generation process discussed in Section 4.2 for the decentralized case is the use of a central server (CS) to compute the partition subgraph for each VPN. These subgraph partitions are distributed to the border node, which uses them to generate the desired TA to be flooded to the VPN CE nodes.

Generating TA for VPNs in a centralized manner follows the same methodology as discussed in Section 4.2, except for the following differences. With reference to the pseudo code shown in Fig. 4.1, Step 2 computes the intermediate subgraph $S\left(V_{k}, E_{k}\right)$ called the partition subgraph from the core network from which the abstract topology is 
derived. In a distributed case, $S\left(V_{k}, E_{k}\right)$ is computed locally in the PE node. In a centralized case, Step 2 uses the logical subgraph partitions obtained from the CS.

Fig. 6.1 shows the pseudo code executed by the CS in order to generate the subgraph partitions. The $\mathrm{CS}$, in its procedure to compute the partitions, iterates over all the VPNs in $k \in U$ and computes $S\left(V_{k}, E_{k}\right)$ applying schemes to be discussed in Section 6.2. Once computed, the partition graphs are distributed to the border node set $P_{k}$ for each VPN $k \in U$. The interaction between the CS and the PE nodes can be implemented in a way that the computed partition graph for a given VPN can be obtained periodically with a period equal to the abstract topology refresh interval $R_{k}$ or on an on-demand basis.

The fairness policy applied in the centralized mode is the same as the policy discussed in Section 4.2.1. Without loss of generality, we assume that the VSP adopts a model where the goal is to always expose the available capacity equally to the VPNs that have the same abstract topology type TA-SLA parameter $T_{k}$, while maintaining the desired property that different abstract topology types result in generating service differentiation. This fairness policy is imposed in two phases. First, the graph partition computation phase in Step 1 in Fig. 6.1 ensures that the available link resource considered during the partition is divided equally among the concerned VPNs. The second phase of fairness is applied by each border node $b$ following, Steps 4-6 in Fig. 4.1 (Stage 2), during the TA generation process as discussed in Section 4.2.1. 


\section{Generate Partition Subgraph for each VPN}

Input: $G(V, E), U, P_{k} \forall k \in U$.

Output: $S\left(V_{k}, E_{k}\right) \forall k \in U$.

begin

//Generate subgraph partition $S\left(V_{k}, E_{k}\right) \forall k \in U$

for each VPN $k \in U$,

Step 1: compute $S\left(V_{k}, E_{k}\right)$ for VPN $k$ applying VPN core capacity sharing algorithms ;

for each VPN $k \in U$,

Step 2: distribute $S\left(V_{k}, E_{k}\right)$ to all nodes in $P_{k}$;

end;

Figure 6-1: VPN subgraph partition computation steps executed by the central server

\subsection{VPN Core Capacity Sharing Problem}

The primary challenge in centralized mode of TA generation is to compute the logical VPN subgraph partition computed by the CS in Step 1 in Fig. 6.1 from which the abstract topologies are derived by the border node (Steps 2-3 in Stage 1 of Fig. 4.1). Therefore, the focus of the subsequent discussion is Step 1 in Fig. 6.1 executed by the CS.

\subsubsection{Objectives of VPN Core Capacity Sharing Problem}

First, the goal of a TA service is to enable better call performance to the VPNs making dynamic capacity requests; in other words, the goal is to maximize call performance. We use the VPN success ratio as a parameter to measure VPN call performance. This 
parameter is a measure of making right bandwidth request decisions by a VPN using the abstraction provided to it by the VSP. This includes the calls that are computed and signaled successfully by the CE node as well those rejected by the VPN locally. The call rejections happen either because of an error between the abstraction and the core state of the network or due to insufficient resources to accommodate the VPN call request because of increased load conditions.

The second objective is to ensure the best possible use of the VSP's available core capacity resource. Hence, the objective is also to maximize a VSP's network utilization. This also correlates with the goal of maximizing the revenue generated out of the TA service.

The third criterion we consider while computing subgraphs for the VPNs is to ensure that the schemes are fair to all the VPNs with respect to the fairness policy discussed in Section 6.2.

Considering these objectives, we define the VPN core capacity sharing problem as follows:

\section{VPN Core Capacity Sharing Problem (VPN-CS):}

Given a graph $G(V, E)$ representing the VSP's core network providing topology abstraction service to the set of VPNs, $U$, the objective is to compute fair partitions $S\left(V_{k}, E_{k}\right)$ for each $V P N k \in U$ of the network so as to maximize the VPN call success ratio and core network utilization. If $x_{e}^{k}$ represents the resource identified as part of subgraph $S\left(V_{k}, E_{k}\right)$ on edge $e \in E$ and for $V P N k \in U$, then $\sum_{k \in U} x_{e}^{k} \leq t(e), \forall e \in E$ must be satisfied. 
The objectives related to maximizing the VPN success ratio and network utilization stated as part of the VPN-CS problem could be positively or negatively correlated depending on the nature of logical subgraph partitioning algorithm applied to solve the VPN-CS problem.

The positive correlation can be explained as follows: Improved network utilization can be achieved by maximizing the bandwidth considered during the partitioning phase, resulting in efficient and non-conservative exposure of the core capacity to the VPNs, and thereby, positively influencing the success ratio of a VPN. On the other hand, a conservative exposure could lead to many calls being terminating locally at the VPN's CE nodes, degrading both the VPN success ratio and network utilization. The objectives of maximizing network utilization and the VPN success ratio may be negatively correlated when the algorithm used to generate subgraphs is aggressive, i.e. when the total capacity abstracted is more than the available resource. In this case, the VPNs are encouraged to seek more resources leading to good network utilization but also leading to an increased contention of available core resource, which may result in poor VPN call performance. Hence, a good solution to the VPN-CS problem has to strike a balance between the two objectives, which are to improve network utilization without decreasing the VPN's success ratio. 


\subsubsection{Multicommodity Flow Formulation of the VPN-CS Problem}

In view of the relationship between the maximization objectives of the VPN-CS problem and the total capacity considered during the partitioning phase (that is, the sum of the link capacities of the partition graphs $S\left(V_{k}, E_{k}\right)$ for each $\left.k \in U\right)$, we next present a multicommodity flow formulation that maximizes the total abstracted capacity. The fairness criteria discussed in Section 6.2 is also incorporated in this formulation.

In this formulation, $K_{v}$ represents the set of all source-destination pairs of VPN $v \in U$ derived from the set $P_{v}$. We define the set of commodities $K$ as the set of all the VPN commodities, i.e. $K=\left\{K_{v} \mid \forall v \in U\right\}$. Let the net flow achieved for each sourcedestination commodity $k \in K_{v}$ of VPN $v$ be $f_{v, k}$. The variable $x_{i, j}^{v, k}$ denotes the logical partition resource assigned to source-destination pair $\left(s_{k}, d_{k}\right) \in K_{v}$ on an edge $(i, j) \in E$. Using these definitions, the VPN-CS problem can be formulated as in (6.1)-(6.5) with the objective of maximizing the aggregate resources considered as part of the subgraph partitions. 


\section{Formulation for VPN Core Capacity Sharing Problem:}

$$
\text { Maximize } \sum_{v \in U} \sum_{k \in K_{v}} \sum_{(i, j) \in E} x_{i, j}^{v, k}
$$

Subject to:

$$
\begin{aligned}
& \sum_{(i, j) \in E} x_{i, j}^{v, k}-\sum_{(j, i) \in E} x_{j, i}^{v, k}=f_{v, k} \forall v \in U, \forall k \in K_{v}, i=s_{k} \\
& \sum_{(i, j) \in E} x_{i, j}^{v, k}-\sum_{(j, i) \in E} x_{j, i}^{v, k}=0 \quad \forall v \in U, \forall k \in K_{v}, i \neq s_{k}, i \neq d_{k} \\
& \sum_{(i, j) \in E} x_{i, j}^{v, k}-\sum_{(j, j) \in E} x_{j, i}^{v, k}=-f_{v, k}, \forall v \in U, \forall k \in K_{v}, i=d_{k} \\
& \sum_{v \in U} \sum_{k \in K_{v}} x_{i, j}^{, v, k} \leq t_{i, j} \quad \forall(i, j) \in E \\
& f_{v 1, k}-f_{v 2, k}=0 \quad \forall\left(s_{k}, d_{k}\right) \in B, \forall(v 1, v 2) \in Z\left(s_{k}, d_{k}\right) \\
& x_{i, j}^{v, k} \geq 0, f_{v, k} \geq 0
\end{aligned}
$$

In the formulation, (6.1)-(6.3) enforce the supply-demand conservation condition for each VPN commodity. (6.4) enforces the capacity constraint for each edge of the core graph. (6.5) enforces the fairness constraint during the subgraph partition computation process with the goal of sharing resources equally among all the VPNs. We define $Z(s, d)$ as the set of VPNs having a common source-destination border node pair $(s, d)$. The constraint (6.5) ensures equal sharing of resources by enforcing the aggregate flow, i.e $f_{v 1, k}, f_{v 2, k}$ for any two VPNs $(v 1, v 2) \in Z\left(s_{k}, d_{k}\right)$ to be equal. The optimum flow values $x_{i, j}^{v, k}$ define the capacity of the link $(i, j)$ in the partition graphs $S\left(V_{v}, E_{v}\right)$ for each $v \in U$. The worst case scenario of the formulation can be assessed assuming that all the VPNs in $U$ are hosted on all the border nodes in $B$. In this case, the number of variables in the formulation is $O\left(|E|^{*}|U|^{*}|B|^{2}+|U|^{*}|B|^{2}\right)$, and the number of possible constraints would be 
in the order of $O\left(|U|^{*}|B|^{2} *|V|+|U|^{2} *|B|^{2}\right.$ ). Since the goal of the TA service is to enable dynamic bandwidth requests based on a topology abstraction in smaller timescales, solving the problem to optimality for large numbers of variables and constraints as in the above formulation using linear programming tools will not be efficient.

Instead, we propose solutions based on a variant of the multicommodity flow problem, namely, the maximum concurrent flow (MConF) problem, whose objective is to maximize the aggregate commodity flow, while ensuring fairness among commodity flows. We later show the possible improvement that could be made to the MConF based solution by adapting a solution based on the maximum multicommodity flow (MMCF) theory. One rationale for using these multicommodity flow variants is to take advantage of the existing fully polynomial-time approximation schemes (FPTAS) from the literature.

We next discuss the formulation of the VPN-CS problem.

\subsubsection{Maximum Concurrent Flow Based Formulation of the VPN-CS Problem}

The MConF problem is defined as follows: Given a network $G(V, E)$ and a set $K$ of source-destination pairs $\left(s_{i}, d_{i}\right), i=1,2, \ldots|K|$, assume that demands $D\left(s_{i}, d_{i}\right) \forall i=1,2, \ldots|K|$ are known. The objective of the MConF problem is to maximize the factor $\beta$ such that there exists a flow that satisfies the demand $\beta^{*} D\left(s_{i}, d_{i}\right), \forall i=1,2, \ldots k$. The node-link formulation of the MConF problem is as follows: 


\section{Maximum Concurrent Flow (MConF) Formulation:}

Maximize $\beta$

Subject to:

$$
\begin{aligned}
& \sum_{(i, j) \in E} x_{i, j}^{k}-\sum_{(j, i) \in E} x_{j, i}^{k}=\beta^{*} D\left(s_{k}, d_{k}\right) \quad \forall k \in K, i=s_{k} \\
& \sum_{(i, j) \in E} x_{i, j}^{k}-\sum_{(j, i) \in E} x_{j, i}^{k}=0 \forall k \in K, i \neq s_{k}, i \neq d_{k} \\
& \sum_{(i, j) \in E} x_{i, j}^{k}-\sum_{(j, i) \in E} x_{j, i}^{k}=-\beta^{*} D\left(s_{k}, d_{k}\right) \quad \forall k \in K, i=d_{k} \\
& \sum_{k \in K} x_{i, j}^{k} \leq t_{i, j} \quad \forall(i, j) \in E \\
& x_{i, j}^{k} \geq 0, \beta \geq 0
\end{aligned}
$$

The value of $\beta$ is called the throughput of the maximum concurrent flow formulation.

The objective of the MConF problem enforces fairness by satisfying the same fraction of the demand for all the commodities, which is also in line with the fairness policy discussed in Section 6.2, which is to share the resources equally among all the VPN commodities.

With regard to the solution for the MConF problem for online implementation, [Sha90] proposed the first approximation algorithm for the MConF problem for networks with equal demands and undirected edges. Several researchers have since proposed enhancements to this algorithm in order to improve its complexity on the generalized version of the problem with arbitrary capacities. Recently, Garg and Konemann [Gar98] proposed a simple approximation algorithm to the MConF problem. Later, Fleischer [Fle99] presented improvements to the maximum concurrent flow approximation algorithm proposed in [Gar98], with better running complexity, particularly, when the 
graph is sparse and has a larger number of commodities satisfying the condition $|K|>$ $|E| / \mid \eta$.

Applying MConF to VPN-CS problem is not straight forward for two reasons. First, the commodity demands $D\left(s_{k}, d_{k}\right)$ for the VPN-CS problem are not known apriori. Secondly, if the commodity is defined for each VPN $v \in U$ and source-destination commodity $k \in K_{v}$, the complexity of the number of commodities would be $O\left(|U|^{*}|B|^{2}\right)$, which may not be scalable for online implementation.

We address these two concerns as follows: As one of the objectives of VPN-CS problem is to maximize the link resources considered during the partitioning phase, we initialize $D\left(s_{k}, d_{k}\right)$ to $\alpha(k)$, where $\alpha(k)$ is the maximum flow for commodity $k$. In order to reduce the complexity of the execution time and memory requirement, we propose a two step optimization approach. The first step is to apply the MConF formulation on an aggregated set of source-destination pairs. For this scheme, we define a commodity $k$ in the aggregated version as source-destination border node pair $(s, d)$ that has the potential VPN bandwidth request from $s$ to $d$. This is the case if the source-destination border nodes $s$ and $d$ host at least one common VPN; in this case, it will satisfy the criteria $\left|\left(U_{s} \cap U_{d}\right)\right| \geq 1$. This aggregation of commodities reduces commodity complexity to $O\left(|B|^{2}\right)$. With this simplification, we propose a centralized heuristic to be executed by the CS in order to solve the VPN-CS problem as shown in the pseudo code in Fig. 6.2. 


\section{Maximum Concurrent Flow Based Partitioning Scheme}

Input: $G(V, E), B, U, P_{k} \forall k \in U$.

Output: $S\left(V_{x}, E_{x}\right), x \in U$.

begin

Step 1: for each potential pair $(s, d), s, d \in B$, identify the set of VPNs $Z(s, d)$ and define commodity set $K=\{(s, d):|Z(s, d)| \geq 1\}$;

Step 2: for each commodity pair $\left(s_{k}, d_{k}\right), \forall k \in K$, compute the max-flow $\alpha(k)$;

Step 3: for each border node pair $\left(s_{k}, d_{k}\right), k \in K$, the supply for $s_{k}$ is set to $\alpha(k)$ and demand at $d_{k}$ is set to - $\alpha(k)$;

Step 4: solve the maximum concurrent flow problem for commodity set $K$ with respect to graph $G(V, E)$; (Note: The solution $x_{i, j}^{k}$ represents the resource associated with commodity $k$ over edge $(i, j) \in E$ )

Step 5: for each commodity pair $\left(s_{k,}, d_{k}\right) \in K$, for each $(i, j) \in E$, if $x_{i, j}^{k}>0$ then set $x_{i, j}^{v, k}=x_{i, j}^{k} /\left|Z\left(s_{k}, d_{k}\right)\right|$; (Note: we divide the edge flows fairly among the VPNs in the set $Z\left(s_{k}, d_{k}\right)$. Here, $x_{i, j}^{v, k}$ is the capacity assigned to VPN $v$ on link $(i, j)$ for VPN source-destination pair $\left.\left(s_{k,}, d_{k}\right) \in K_{v}\right)$

Step 6: for all VPN $v \in U$, create partition subgraph $S\left(V_{v}, E_{v}\right)$, where $V_{v} \subset V$ and $E_{v} \subset E$ are the set of all links such that $x_{i, j}^{v, k}>0$ for at least one commodity $k \in K_{v}$ of VPN $v$. The capacity of each link $(i, j)$ in $S\left(V_{v}, E_{v}\right)$ is the sum of the flows $x_{i, j}^{v, k}$ for all commodities $k \in K_{v}$; 
Step 7: for each VPN $v$, distribute $S\left(V_{v}, E_{v}\right)$ to the border nodes in $P_{v}$;

end;

Figure 6-2: MConF based partitioning heuristic for VPN core capacity sharing problem

Based on our previous discussion, Steps 1-4 are self explanatory. Step 5 iterates over each commodity $k \in K$, and for each commodity $k$ and the set of VPNs $Z\left(s_{k}, d_{k}\right)$ sharing the source-destination pair, we assign the logical capacity for VPN $v \in Z\left(s_{k,}, d_{k}\right)$ over link $(i, j)$, denoted as $x_{i, j}^{v, k}$, by dividing the MConF edge flow $x_{i, j}^{k}$ equally among all the VPNs $v \in Z\left(s_{k,}, d_{k}\right)$. This step is inline with the fairness policy discussed in Section 4.2.1. Step 6 uses the capacities logically partitioned for each of the VPN $v \in U$ on each link $(i, j) \in E$ in order to realize the VPN subgraph $S\left(V_{v}, E_{v}\right)$, which is obtained by constructing a subgraph with positive edge flows assigned for VPN $v$. Once $S\left(V_{v}, E_{v}\right)$ is determined, it is then distributed to the nodes in $P_{v}$ (those border nodes hosting VPN $v$ ) in Step 7.

The partition subgraphs will be used by the PE nodes in Step 3 of the TA process (Fig. 4.1), discussed in Section 4.2, in order to generate a fully meshed abstract graph $G_{k, F M A}\left(V_{k}, E_{k}\right)$. To generate $G_{k, F M A}\left(V_{k}, E_{k}\right)$, we can apply one of the algorithms discussed in Chapter 5. The most efficient algorithm in this case would be the maximum capacity scheme; this is because no two VPN partition graphs have any overlapping resources between them. For online implementation, [Fle99] (Appendix A.6) proposes the fastest $\varepsilon$ approximation algorithm for the MConF problem; this can be applied in Step 4 of the 
MConF based heuristic. With respect to the complexity of the MConF based partitioning scheme, it is dominated by Steps 2 and 4, which subsequently results in the overall complexity of $O\left(\varepsilon^{-2}|E|^{*}(|E|+|K|)+|K|^{*}|V|^{3}\right)$.

\subsubsection{Maximum Multicommodity Flow Formulation of the VPN-CS Problem}

We now draw attention to a drawback of the MConF based partitioning scheme and propose an approach based on the maximum multicommodity flow (MMCF) theory in order to address it. Fig. 6.3 shows a VSP core network. Let us consider three commodities $k(l)=\left\{P E_{1}, P E_{4}\right\}, k(2)=\left\{P E_{1}, P E_{2}\right\}$, and $k(3)=\left\{P E_{3}, P E_{2}\right\}$.

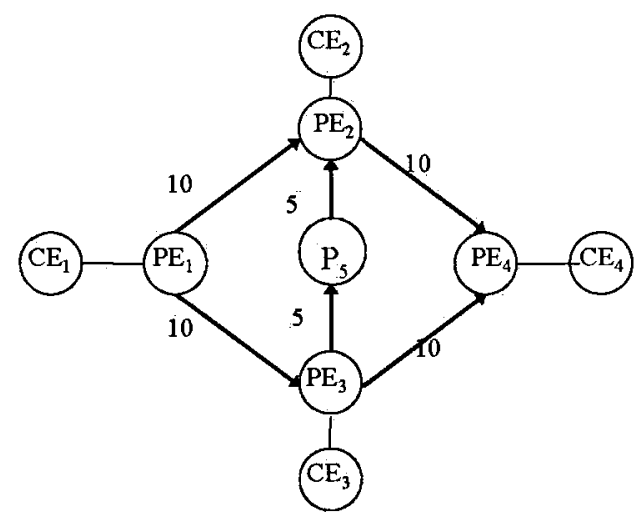

Figure 6-3: Example demonstrating MConF drawback

The throughput achieved by the MConF formulation with the values of the commodities set to their maximum flow values of $\{20,15,5\}$ is $\beta=0.5$, and the aggregate abstracted capacity (which is the sum of flows in all the links of all the partition graphs, for this 
example the only VPN) is 20 units. This happens because the edges of the paths corresponding to a commodity's maximum flow are also shared by other commodities, which results in $\beta<1$ forcing the same fraction of the demand to be satisfied for all the commodities. Through observation, we can see that the aggregate commodity flow, which can be achieved when the flows of each commodity are increased independently, is 25 units. This inefficiency can be addressed by maximizing the sum of the independent commodity flows; in other words, we use the objective of maximizing $\sum_{k} \beta_{k} * D\left(s_{k}, d_{k}\right)$. The modified MconF (M-MConF) problem is stated next.

Modified Maximum Concurrent Flow (M-MConF) Formulation:

$$
\text { Maximize } \sum_{k} \beta_{k}^{*} D\left(s_{k}, d_{k}\right)
$$

Subject to:

$$
\begin{aligned}
& \sum_{(i, j) \in E} x_{i, j}^{k}-\sum_{(j, i) \in E} x_{j, i}^{k}=\beta_{k}^{*} D\left(s_{k}, d_{k}\right) \forall k \in K, i=s_{k} \\
& \sum_{(i, j) \in E} x_{i, j}^{k}-\sum_{(j, i) \in E} x_{j, i}^{k}=0 \quad \forall k \in K, i \neq s_{k}, i \neq d_{k} \\
& \sum_{(i, j) \in E} x_{i, j}^{k}-\sum_{(j, i) \in E} x_{j, i}^{k}=-\beta_{k} * D\left(s_{k}, d_{k}\right) \quad \forall k \in K, i=d_{k} \\
& \sum_{k \in K} x_{i, j}^{k} \leq t_{i, j} \quad \forall(i, j) \in E \\
& x_{i, j}^{k} \geq 0 \quad \beta_{k} \geq 0
\end{aligned}
$$


We now show that the M-MConF problem is equivalent to the maximum multicommodity flow (MMCF) problem, whose objective is to maximize the total flow for all the commodities. The node-link MMCF formulation is as follows:

\section{Maximum Multicommodity Flow (MMCF) Formulation:}

$$
\text { Maximize } \sum_{k \in K} f_{m c}(k)
$$

Subject to:

$$
\begin{aligned}
& \sum_{(i, j) \in E} x_{i, j}^{k}-\sum_{(j, i) \in E} x_{j, i}^{k}=f_{m c}(k) \quad \forall k \in K, i=s_{k} \\
& \sum_{(i, j) \in E} x_{i, j}^{k}-\sum_{(j, i) \in E} x_{j, i}^{k}=0 \forall k \in K, i \neq s_{k}, i \neq d_{k} \\
& \sum_{(i, j) \in E} x_{i, j}^{k}-\sum_{(j, i) \in E} x_{j, i}^{k}=-f_{m c}(k) \forall k \in K, i=d_{k} \\
& \sum_{k \in K} x_{i, j}^{k} \leq t_{i, j} \quad \forall(i, j) \in E \\
& x_{i, j}^{k} \geq 0 \quad f_{k} \geq 0
\end{aligned}
$$

Here, $f_{m c}(k)$ is the aggregate MMCF flow variable corresponding to commodity $k$. The objective of MMCF is to maximize the total commodity flow. As in previous formulations, (6.14)-(6.16) represent the supply-demand constraints and (6.17) the capacity bound for edge $(i, j) \in E$.

To show the equivalence of the MMCF and M-MConF formulations, let $\beta_{k}=f_{m c}(k) / D\left(s_{k}, d_{k}\right)$ for M-MConF problem, where $f_{m c}(k)$ are the optimal commodity flows achieved by solving the MMCF problem. With this the aggregate M-MConF flow is 
$f_{m m}=\sum_{k \in K} \beta_{k} * D\left(s_{k}, d_{k}\right)$. We can then see that the flows achieved by the MMCF problem form a feasible solution to the M-MConF problem. Therefore,

$$
f_{m c} \leq f_{m m}
$$

where $f_{m c}$ is the optimal aggregate commodity flow value for the MMCF problem. On the other hand, setting each $f_{m c}(k)=\beta_{k}{ }^{*} D\left(s_{k}, d_{k}\right)$, where $\beta_{k}$ 's are the optimal commodity throughputs obtained by solving the M-MConF formulation, we can see that the commodity flow values corresponding to an optimum solution of the M-MConF problem form a feasible solution to the MMCF problem for the same network and set of commodities. Hence, we have

$$
f_{m m} \leq f_{m c}
$$

From (6.18) and (6.19), we conclude:

$$
f_{m m}=f_{m c}
$$

Thus, the aggregate flow achieved by MMCF problem is equal to that achieved by solving the M-MConF problem. So, we expect that the MMCF and the equivalent M-MConf approach will overcome the conservative nature of $\mathrm{MConF}$, and this will result in both improved network utilization and VPN success ratio.

Next, we propose a MMCF based heuristic to solve the VPN-CS problem as follows in Fig. 6.4: 


\section{Maximum Multicommodity Flow Based Partitioning Scheme}

Input: $G(V, E), B, U, P_{k} \forall k \in U$.

Output: $S\left(V_{x}, E_{x}\right), x \in U$.

begin

Step 1: for each potential border node pair $(s, d)$, identify the set $Z(s, d)$. Define commodity set $K=\{(s, d):|Z(s, d)|>0\}$;

Step 2: solve the maximum multicommodity flow problem for commodity set $K$ with respect to graph $G(V, E)$; (Note: The solution $x_{i, j}^{k}$ represents the resource associated with commodity $k$ over edge $(i, j) \in E$ )

Step 3: for each commodity pair $\left(s_{k}, d_{k}\right) \in K$, for each $(i, j) \in E$, if $x_{i, j}^{k}>0$ then set $x_{i, j}^{v, k}=x_{i, j}^{k} /\left|Z\left(s_{k}, d_{k}\right)\right|$; (Note: we divide the edge flows fairly among the VPNs in the set $Z\left(s_{k}, d_{k}\right)$. Here, $x_{i, j}^{v, k}$ is the capacity assigned to VPN $v$ on link $(i, j)$ for VPN source-destination pair $\left.\left(s_{k,}, d_{k}\right) \in K_{v}\right)$

Step 4: for all VPN $v \in U$, create partition subgraph $S\left(V_{v}, E_{v}\right)$, where $V_{\nu} \subset V$ and $E_{v} \subset E$ are the set of all links such that $x_{i, j}^{v, k}>0$ for at least one commodity $k \in K_{v}$ of VPN $v$. The capacity of each link $(i, j)$ in $S\left(V_{v}, E_{v}\right)$ is the sum of the flows $x_{i, j}^{v, k}$ for all commodities $k \in K_{v}$;

Step 5: for each VPN $v$, distribute $S\left(V_{v}, E_{v}\right)$ to the border nodes in $P_{v}$; end;

Figure 6-4: MMCF based partitioning heuristic for VPN core capacity sharing problem 
As in the case of the MConF based partition generation scheme, MMCF begins in Step 1 by initializing the set $Z(s, d)$ for all potential source-destination pairs $(s, d) \in B$; this is then used to determine the commodity set $K$. Step 2 solves the MMCF problem. For an online implementation, the $\varepsilon$-approximation algorithm from [Fle99] (Appendix A.5) can be applied. This results in partitions associated with aggregate source-destination commodity $x_{i, j}^{k} \forall k \in K$ and $(i, j) \in E$. Steps 3-5 are similar to the fair partitioning process discussed with respect to the MConF based partitioning solution. The MMCF heuristic is expected to achieve better TA performance compared to MConF heuristic; this is due to an increase in aggregate edge capacity considered during the partitioning process. For an online implementation, the overall complexity of this heuristic is dominated by Step 2, where we apply the $\varepsilon$-approximation algorithm presented in [Fle99], which results in a complexity of $O\left(\varepsilon^{-2}|E|\left(|E|+|V|^{*} \log |E|\right) \log (|V|)\right)$.

\subsection{Improving Fairness of MMCF Based Partitioning Heuristic}

Though the MMCF based partitioning scheme maximizes the aggregate flow, it may not achieve the desired objective of maximizing $\beta_{k}$ for each commodity $k$ in a fair manner. Hence, the final $\beta_{k}$ values may end up being very optimistic for a few commodities and conservative for others, which leads to unfair resource partitioning among the commodities. Since the goal of maximizing the VPN success ratio also requires partitioning the network capacity as fairly as possible among all the VPNs, we define 
another problem called the fair partitioning problem, whose goal is to improve the faimess of the commodity flows obtained by using the MMCF based partitioning scheme. This problem can be stated as follows:

\section{Fair Partitioning Problem}

Given a solution for the VPN-CS problem with individual commodity flows $f_{m c}(k) \forall k \in K$, the fair partitioning problem is intended to rearrange the path flows for each commodity so as to minimize $\left|f_{m c}(i) / \alpha(i)-f_{m c}(j) / \alpha(j)\right| \forall i, j \in K$.

The goal of the fair partitioning problem is to minimize the difference between the ratio of flow achieved from the MMCF solution and the maximum flow between any pair of commodities. In order to achieve this, we propose modified MMCF formulations called the bounded MMCF formulations suitable for offline implementation and a flow balancing algorithm for online implementation in dynamic environments.

Both the offline and online improvements to be proposed will be based on balancing commodity flows obtained from MMCF based heuristic. We begin by first dividing the set of commodities $K$ into the deficit set $\left(\Omega_{d}\right)$ and the excess set $\left(\Omega_{e}\right)$ based on the fraction of maximum flow $\alpha\left(s_{k}, d_{k}\right)$ achieved by the MMCF solution as discussed next. The sets are derived by first normalizing the aggregate MMCF commodity flow $f_{m c}(k)$ with its corresponding maximum flow $\alpha(k)$. From this, the set $S_{K}=\left\{f_{m c}(k) / \alpha(k), \forall k \in K\right\}$ is defined. We next define a threshold value $\sigma$ as follows: Let $S_{\min }=\min \left\{S_{K}\right\}$ and $S_{\max }=\max \left\{S_{K}\right\} . \sigma$ is set to $\left(S_{\min }+S_{\max }\right) / 2$. The threshold $\sigma$ is then used 
to divide set $S_{K}$ into sets $\Omega_{e}$ and $\Omega_{d}$ as follows: $\Omega_{d}=\left\{k \mid k \in K, S_{k} \leq \sigma\right\}$, $\Omega_{e}=\left\{k \mid k \in K, S_{k}>\sigma\right\}$. Applying this definition of deficit and excess commodity sets, we next discuss the bounded MMCF formulation and the flow balancing algorithm in order to address the fair partitioning problem.

\subsubsection{Bounded MMCF Formulation}

This formulation retains the objective of the MMCF formulation but adds more constraints in the form of lower and upper bounds to the excess and deficit commodity elements with the goal of reducing the imbalance among commodity flows. Introducing such bounds enables us to achieve a minimum level of faimess for each deficit commodity without allowing the flows of the excess commodities to fall below a certain threshold flow. We represent the upper and lower bound flows of commodity $e \in \Omega_{e}$ as $\varphi_{e, l}$ and $\varphi_{e, u}$ respectively, and those for commodity $d \in \Omega_{d}$ as $\varphi_{d, l}$ and $\varphi_{d, u}$. The modified MMCF formulation applying the new constraints can be stated as follows: 
Bounded MMCF formulation for Fair Partitioning Problem:

$$
\text { Maximize } \sum_{k \in K} f_{b}(k)
$$

Subject to:

$$
\begin{aligned}
& \sum_{(i, j) \in E} x_{i, j}^{k}-\sum_{(j, i) \in E} x_{j, i}^{k}=f_{b}(k) \forall k \in K, i=s_{k} \\
& \sum_{(i, j) \in E} x_{i, j}^{k}-\sum_{(j, i) \in E} x_{j, i}^{k}=0 \quad \forall k \in K, i \neq s_{k}, i \neq d_{k} \\
& \sum_{(i, j) \in E} x_{i, j}^{k}-\sum_{(j, i) \in E} x_{j, i}^{k}=-f_{b}(k) \forall k \in K, i=d_{k} \\
& \varphi_{e, l} \leq f_{b}(e) \leq \varphi_{e, u} \quad \forall e \in \Omega_{e} \\
& \varphi_{d, l} \leq f_{b}(d) \leq \varphi_{d, u} \quad \forall d \in \Omega_{d} \\
& \sum_{k} x_{i, j}^{k} \leq t_{i, j} \quad \forall(i, j) \in E \\
& x_{i, j}^{k} \geq 0 \quad f_{b}(k) \geq 0
\end{aligned}
$$

In the formulation, $f_{b}(k)$ is the aggregate commodity balanced flow resulting from solving the problem. As can be seen from this formulation that the new constraints, i.e. (6.24) and (6.25), are the lower and upper bound constraints on the flows of the excess and deficit commodities, whose initialization we discuss next.

\subsubsection{Lower and Upper Bound Initialization for Bounded MMCF Formulation}

The task now is to understand the initialization of these bounds. Care should be taken to define the bounds, so that the solution obtained by the modified bounded MMCF formulation is at least as good as the one obtained by solving the MMCF formulation. 
We propose two ways to initialize the lower and upper bounds of the aggregate flow of the commodities in the sets $\Omega_{e}$ and $\Omega_{d}$.

In the first approach, which we identify as MB-1 formulation, we assume that the maximum flows and MMCF flows for the commodities in set $K$ with respect to the graph $G(V, E)$ are known. In this case, the lower bound $\varphi_{e, l}$ for the excess commodity element $e \in \Omega_{e}$ is set so that its aggregate flow does not become less than $\sigma^{*} \alpha(e),(\alpha(k)$ is the maximum flow of commodity pair $\left(s_{k}, d_{k}\right)$ ), and the upper bound $\varphi_{e, u}$ does not exceed the flow obtained from the MMCF solution, which is $f_{m c}(e)$. In case of the deficit commodity elements $d \in \Omega_{d}$, the lower and upper bound settings are reversed. In this case, the lower bound $\varphi_{d, l}$ is set so that the flow achieved is at least $f_{m c}(d)$, and the upper bound $\varphi_{d, u}$ is set so that it does not exceed the desired threshold $\sigma^{*} \alpha(d)$.

The second proposal to initialize the bounds which we identify as the MB-2 formulation is based on the observation that the optimal commodity flow solution of a MConF problem, which ensures fairness through its throughput factor $\beta$, is also a feasible MMCF solution. For the MB-2 formulation, we must first determine the throughput factor $\beta$ by solving the MConF problem with the demands set to maximum flow of the commodity $k, \alpha(k)$. Once the MConF flows are obtained, the lower bound $\varphi_{e, l}$ of excess commodity $e \in \Omega_{e}$ is set to $\beta^{*} \alpha(e)$ and upper bound $\varphi_{e, u}$ to $f_{m c}(e)$. For the commodity elements in $d \in \Omega_{d}$, we set lower bound $\varphi_{d, l}$ to $\beta^{*} \alpha(d)$, and the upper bound $\varphi_{d, u}$ is set to $\alpha(d)$. We study the performance of the bounded MMCF formulations in the performance analysis section in Section 6.5 . 
The above changes to the MMCF formulation are expected to improve fairness among the commodity flows, but as stated earlier, the formulations would have to be solved in a offline manner using an LP tool, which would make the approach too complex to be realized in a dynamic environments. So, we next discuss a heuristic for online implementation.

\subsubsection{Flow Balancing Heuristic}

This algorithm builds on the approach discussed in the previous section where the set of commodities $K$ is first divided into an excess set $\Omega_{e}$ and a deficit set $\Omega_{d}$. The two sets are determined similarly to the approach discussed earlier using the threshold factor $\sigma$. The algorithm builds on the idea of transferring flows from the elements in excess commodity set $\Omega_{e}$ to the elements in deficit commodity set $\Omega_{d}$. The algorithm has as inputs the MMCF flow $f_{m c}(k)$ and the corresponding path set $P_{k}$ for commodity $k \in K$, and it outputs the modified balanced flows $f_{b}(k)$ for commodities in the set $K$. The flow balancing algorithm is explained next with reference to Fig. 6.5 and the pseudo code in Fig. 6.6.

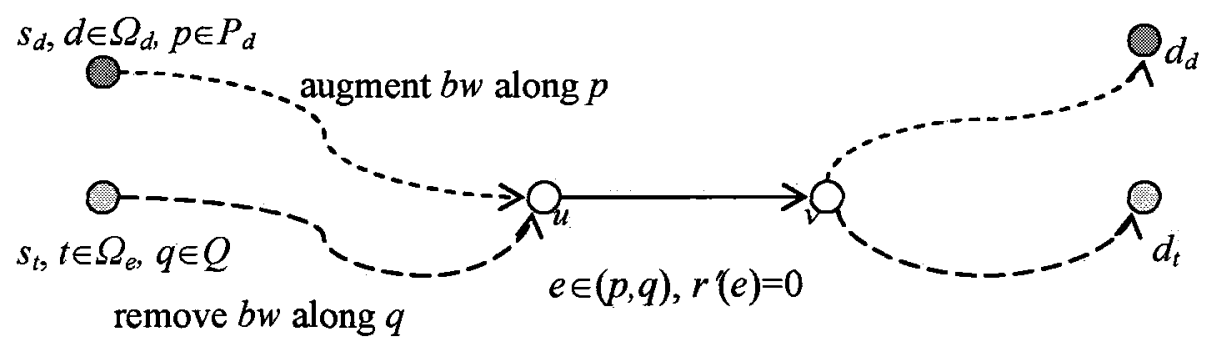

Figure 6-5: Logical view of the flow balancing process 
In Steps 1-2, the algorithm begins by identifying the sets $\Omega_{e}$ and $\Omega_{d}$ and initializing $f_{b}(k)$ to the previously computed $f_{m c}(k)$. Flow balancing begins by iterating through each deficit commodity item $d \in \Omega_{d}$ in Step 3. In Step 4, for each commodity $d$, we iterate over all the paths $p \in P_{d}$ corresponding to the commodity flow $f_{b}(d)$. We choose a path $p=\left\{S_{d,} e_{1}, e_{2}, . . e, . . D_{d}\right\}$ for further steps in the iteration only if it has exactly one edge $e$ such that the left over edge capacity after executing MMCF based partitioning algorithm, which we denote as $r(e)$, satisfies $r(e)=0$, and $r\left(e_{i}\right) \geq \tau$ for all other edges $e_{i} \in p$ and $e_{i} \neq e$, for a pre-defined left over edge capacity threshold $\tau$. We impose this condition for three reasons: First, the edge with the zero left over capacity increases the probability of finding at least one or more commodities from the $\Omega_{e}$ set contributing to the total edge flow, and this could be used to potentially transfer flow to the deficit commodity; second, this approach enables us to control the complexity of the algorithm by limiting the choice of edges that can be chosen on a path $p \in P_{d}$, and thereby, simplifying the choice of the excess commodity from which the flow can be transferred; third, $\tau$ also acts like a tunable parameter that could also be used to control the complexity of the algorithm by eliminating the paths that may not make a considerable change to the flow of the deficit commodity after an augmentation iteration. If an FPTAS algorithm was used to solve the MMCF problem, and as the optimality of the solution is a function of the approximation factor, the left over capacity on all the edges of the path $p$ may be positive. In this case, we chose the path $p$ and an edge $e$ such that $e \in\{x: \min \{r(x), x \in p\}\}$ and with left over capacity $r\left(e_{i}\right)>\tau, \forall e_{i} \in p$ and $e_{j} \neq e$. 
Once a path $p$ is determined, we determine in Step 5 the set $T \subset \Omega_{e}$ of excess commodities that can potentially contribute flows to the deficit commodity $d$. In Step 6, we select an excess commodity $t \in T$ that can contribute the maximum units of flow. We choose $t \in T$, which maximizes $\left(f_{b}(t) / \alpha(t)-\sigma\right)$, i.e $t \in\left\{x: \max \left\{f_{b}(x) / \alpha(x)-\sigma\right\} \wedge x \in T\right\}$. In Step 7, corresponding to the commodity $t$, we identify the subset $Q$ of commodity path set $P_{t}$ that includes edge $e$. From the set $Q$, we choose the path $q \in Q$ that maximizes the flow of commodity $t$ on path $q$; we represent this flow as $\lambda_{t, q}$. In Step 8 , to determine the flow that can be transferred from the excess commodity $t$ to the deficit commodity $d$, we compute the following:

- $\lambda_{t, q}=$ Minimum of the flows of the excess commodity $t$ on the edges of path $q$.

- $\zeta_{d, p}=$ Minimum of the left over capacities of the links on path $p$ of the deficit commodity $d$ without considering the left over capacity of the link $e$.

- $f_{d}=\left(\sigma^{*} \alpha(d)-f_{b}(d)\right)$ is the flow required for deficit commodity $d$ in order to achieve its target flow threshold, which is $\sigma^{*} \alpha(d)$.

- $f_{t}=\left(f_{b}(t)-\sigma^{*} \alpha(t)\right)$ is the maximum flow that can be contributed by commodity $t$ beyond which the flow of the excess commodity would go below the threshold flow $\sigma^{*} \alpha(t)$.

We choose the minimum of the above values in order to determine $b w$, i.e $b w=\min \left\{\lambda_{t, q}, \zeta_{d, p},\left(\sigma^{*} \alpha(d)-f_{b}(d)\right),\left(f_{b}(t)-\sigma^{*} \alpha(t)\right)\right\}$. In Step $9, b w$ is deducted from edge flows corresponding to the excess commodity $t$ on path $q$ and augmented along the edges of path $p$ of the deficit commodity $d$. Corresponding augmentation and subtraction are 
also made to respective aggregate commodity flows $f_{b}(d), f_{b}(t)$, and set $\Omega_{e}$ is updated if necessary. If $\Omega_{e}$ is empty, we terminate, else this process continues over all the deficit commodities until either each of the deficit commodities $d \in \Omega_{d}$ satisfies $\left(f_{b}(d) / \alpha(d)\right) \geq \sigma$, or there are no more paths $p$ for augmentation in the set $P_{d}$ in the iteration for commodity $d$, upon which we move on the next deficit commodity in the set $\Omega_{d}$.

\section{Flow Balancing Heuristic for Flow Partitioning Problem}

Input: $G(V, E), K, f_{m c}(k), \alpha(k), P_{k} \forall k \in K, \sigma, \tau$.

Output: $f_{b}(k) \forall k \in K$ and modified $x_{i, j}^{k}, \forall(i, j) \in E$.

begin

for all commodity $k \in K$, set $f_{b}(k)=f_{m c}(k)$;

Step 1: partition the set of commodities into two sets, $\Omega_{e}$ and $\Omega_{d}$, using threshold factor $\sigma$;

Step 2: identify path set $P_{d}, \forall d \in \Omega_{d}$ and path set $P_{e}, \forall e \in \Omega_{e}$. Initialize for $\forall k \in K$, $f_{b}(k)=f_{m c}(k)$;

Step 3: iterate (Steps 4-10) over each deficit commodity $d, \forall d \in \Omega_{d}$

Step 4: iterate (Steps 5-10) over each path $p \in P_{d}$ with only one saturated link $e \in P_{d}$ in the path, where $e=\{x: \min \{r(x), x \in p\}\}$ and with non-zero left over capacities in the remaining links i.e. $r\left(e_{i}\right)>\tau, \forall e_{i} \in p$ and $e_{i} \neq e$;

Step 5: find the subset $T \subset \Omega_{e}$ of excess commodities having non-zero flow on the link $e \in P_{d}$ 
Step 6: from the set $T$, we determine the commodity $t \in T$, which can contribute maximum additional flow. This is done by choosing the commodity $t$ that maximizes the difference $\left(f_{b}(t) / \alpha(t)-\sigma\right)$;

Step 7: once the excess commodity $t$ is chosen, identify a path set $Q=\left\{x: \forall x \in P_{t}, e \in x\right\}$. From $Q$, choose $q \in Q$ that maximizes the flow of commodity $t$ on path $q$;

Step 8: the amount of flow that can be transferred from excess commodity $t$ to the deficit commodity $d$ is determined by: $b w=\min \left\{\lambda_{t, q}, \zeta_{d, p}, f_{d}, f_{t}\right\}$; (Note: here, $\lambda_{t, q}$ is the flow of commodity $t$ along path $q ; \zeta_{d, p}$ is the minimum of left over capacity of path $p$ corresponding to deficit commodity $d ; f_{d}$ is the flow required for deficit commodity $d$ to achieve its target flow threshold $\sigma^{*} \alpha(d) ; f_{t}$ is the maximum flow that can be contributed by commodity $t$ beyond which the flow of the excess commodity would go below the threshold flow $\left.\sigma^{*} \alpha(t)\right)$.

Step 9: augment flow equal to $b w$ along the deficit commodity path $p$. The equivalent flow is also deduced from excess commodity path $q$. Also, update $f_{b}(d)=f_{b}(d)+b w$ and $f_{b}(t)=f_{b}(t)-b w$. If $\left(f_{b}(t)-\sigma_{t h} * \alpha(t)\right) \leq 0$, update set $\Omega_{e}=\Omega_{e}-\{t\}$

If $\Omega_{e}=0$ then go to end;

Step 10: Check to see if deficit commodity has attained the threshold flow, i.e. $\left(f_{b}(d)-\sigma_{t h} * \alpha(d)\right) \geq 0$. If so, the heuristic proceeds to consider the next deficit commodity (Step 3), else repeats the process for next path in the set $P_{d}$ (Step 4);

end;

Figure 6-6: Flow balancing heuristic for fair partitioning problem 
The complexity of the balancing heuristic can be controlled by limiting the number of paths considered for each commodity in $\Omega_{d}$ and $\Omega_{e}$. The worst case complexity of the flow balancing heuristic can be derived assuming a highly unfair flow distribution by MMCF, where the size of $\left|\Omega_{d}\right|$ is $O(|K|)$. Let $\left|P_{d}\right|$ and $\left|P_{e}\right|$ be the maximum number of paths to be considered for each deficit and excess commodity as part of the balancing process. The worst case complexity of the flow balancing heuristic with this assumption is $O\left(|K|^{*}\left|P_{d}{ }^{*}\right| P_{e} \mid\right)$.

The flow balancing heuristic can be incorporated as part of the MMCF based partitioning heuristic in order to improve the fairness of the MMCF commodity flow by modifying Step 2 (Fig. 6.4) as follows:

Step 2: For the commodity set $K$, solve the maximum multicommodity flow problem. The solution $x_{i, j}^{k}$ represents the resource associated with commodity $k$ over edge $(i, j) \in E$.

Step 2a: If the MMCF fair partitioning scheme is enabled, determine set $S_{K}=\left\{f_{m c}(k) / \alpha(k)\right\}, \forall k \in K$, Initialize $\sigma$

Step 2b: Apply MB-1, MB-2, or flow balancing heuristic in order to obtain new $x_{i, j}^{k}$ 's and balanced MMCF flows $f_{b}(k)$ which is the new $f_{m c}(k)$;

The complexity of the MMCF based partitioning heuristic for the online implementation case with the above changes could be dominated by either the computation of Step 2 execution of MMCF formulation to optimality or $\varepsilon$-approximation algorithm or the $\mathrm{O}(|K|)$ max flows computation and flow balancing algorithm complexity depending on the sizes 
of $\left|\Omega_{d}\right|,\left|P_{d}\right|,\left|P_{e}\right|$. Considering this, the overall pseudo-polynomial complexity using FPTAS for MMCF proposed in [Fle99], with the previously discussed flow balancing algorithm, is $O\left(\varepsilon^{-2}|E|\left(\left(|E|+|V|^{*} \log |E|\right) \log (|V|)\right)+\left(|K|\left(|V|^{3}+\left(\left|P_{d}\right|^{*}\left|P_{e}\right|\right)\right)\right)\right.$.

\subsection{Simulation and Performance Evaluation}

In this section, we study the performance of the proposed partitioning algorithms and their enhancements to address the fairness issue through offline analysis and using a simulation scenario. The simulation study has been broken down into four objectives:

Objective 1: Understanding the criterion and tradeoffs while choosing the value of epsilon ( $\varepsilon$ ) for the $\varepsilon$-approximation FPTAS algorithms to solve MConF or MMCF based resource partitioning schemes.

Objective 2(a-b): In the first sub-objective, we study the performance of the MConF based resource partitioning algorithm. In the second part, we study and compare the performance of the MMCF based resource partitioning algorithm with respect to the MConF based scheme.

Objective 3(a-b): As part of this objective, in the first part, we study the performance with respect to the improvement achieved by the bounded MMCF formulations (MBland MB-2) in terms of fairness and compare their performance to the performance of the MMCF formulation without the fairness constraints. The analysis is done in an offline 
manner using an LP tool [LINDO] over well known service provider networks referred from [Ho07]. In the second part, we present a simulation analysis of the performance of the flow balancing algorithm proposed to improve the fairness of MMCF based partitioning scheme.

Objective 4: The goal of this objective is to compare the performance of the centralized abstraction schemes with the decentralized schemes proposed in the previous chapter. Here, we also study how oversubscription of the residual link capacity can be used to overcome the conservative nature of centralized TA generation schemes to a certain extent. We also study the effect of oversubscription on VPN call performance and network utilization.

\subsubsection{Performance Metrics}

The performance metrics we use to compare the different algorithms are success ratio, crankback ratio, misscall ratio, and network utilization; these metrics were defined in Section 4.3.2. In addition to these metrics, we also use another metric called abstraction efficiency. This performance metric will be used to compare the efficiency of the partitioning schemes in terms of the total residual link capacity considered as part of the logical VPN partition $S\left(V_{k}, E_{k}\right)$ as a result of applying one of the partitioning schemes. This metric is defined as follows: 
Abstraction Efficiency: This metric is the ratio of the sum of all the commodity flows on all the edges that results as a consequence of applying the MConF or the MMCF based partitioning scheme to the sum of the total residual capacities of all the edges.

$$
\text { Abstraction Efficiency }=\frac{\text { Aggregate com modity flow over all links }}{\text { Aggregate capacity of all links }}
$$

\subsubsection{Simulation Setup}

For the objectives that require simulation analysis, MDVF providing TA service to the IP-VPNs was simulated using OPNET [OPNET] on a VSP topologies, shown in Figs. 4.3-4.5. The results discussed in the section have been obtained from 30 independent replications to achieve an absolute error of less than $1 \%$ and C.I of $95 \%$. Each of the PE nodes was configured to handle five different VPN instances. In addition, a central server (CS) that executes the solutions proposed for the VPN-CS problem for generating core partitions was also implemented. The control plane routing and signaling implementation is similar to that described in Section 4.3.2.

The dynamic bandwidth call requests from the VPN client nodes are modeled as Poisson arrivals, with the capacity requested being uniformly distributed between $[1-X]$. Here, $X$ is the upper bound of the uniformly distributed demand. We present our results for different settings of $X$ chosen from the range of $[50,500]$. Similarly, the VPN call holding time $(H)$ is exponentially distributed between $[10,1000] \mathrm{s}$. The load on the network is varied by either varying $X$ while holding $H$ constant, or by varying $H$ while holding $X$ constant. The inter-computation interval of the abstract topologies and the 
abstract topology refresh interval of the TA to VPN $k, T_{k}$ is set so that they are less than the mean inter-arrival time of the calls from the VPNs. For all the objectives, we initialize the mean inter-arrival time and mean call holding time for the all the VPNs to be the same. The abstract topology update interval $T_{k}$ is also initialized to the same value for all the VPNs and is set equal to the mean inter-arrival time of the call requests.

Table 6.7 shows the values of the parameters used in the simulation study for objectives 2 and 3. The simulation statistics were collected for 3000 calls from all the VPNs. The performances are compared by varying the load of the network, which can be varied by either varying the mean call holding time $H$ or by varying the upper bound $X$ of the bandwidth request, while keeping the call arrival rate and mean holding time as constants. 


\begin{tabular}{|c|c|c|c|}
\hline Stmulation Paramerers & obl. 1.3 & $(80)+4(a)$ & $010102(0)$ \\
\hline VPN Mean Call Inter-arrival time (s) & 100 & 100 & 100 \\
\hline VPN Mean Call Holding Time $(H)(s)$ & {$[10,1000]$} & {$[10,1000]$} & {$[50,100]$} \\
\hline VPN Bandwidth Request $(X)$ & {$[1,100]$} & {$[1,100]$} & {$[1,500]$} \\
\hline Core Topology Link state Update Interval (s) & 5 & 5 & 5 \\
\hline CS Abstract Topology Update Interval (s) & 10 & 10 & 10 \\
\hline VPNAbstract Topology Type (VPN (A-E)) & SSA & SSA & SSA \\
\hline Abstract Topology Refresh Interval (VPNA) (s) & 100 & 100 & 100 \\
\hline Abstract Topology Refresh Interval (VPNB) (s) & 100 & 100 & 100 \\
\hline Abstract Topology Refresh Interval (VPNC) (s) & 100 & 100 & 100 \\
\hline Abstract Topology Refresh.Interval (VPND) (s) & 100 & 100 & 100 \\
\hline Abstract Topology Refresh Interval (VPNE) (s) & 100 & 100 & 100 \\
\hline Approximation factor (for MConF/MMCF FPTAS) & 0.7 & 0.7 & 0.7 \\
\hline
\end{tabular}

\section{Table 6.1: Simulation parameters to evaluate centralized abstraction schemes}

\section{Objective 1: Choosing epsilon $(\varepsilon)$ for approximation algorithms for centralized}

\section{subgraph partitioning heuristics}

One of the inputs when applying the FPTAS approximation algorithms for the MConF and MMCF problems is the choice of value for $\varepsilon$. In the context of these problems which has an objective of maximizing commodity flows, $\varepsilon$ is the approximation factor which when input to a MConF or MMCF FPTAS provides a solution that is at least $(1-\varepsilon)$ factor of the optimal solution.

The choice of value of $\varepsilon$ can be determined by understanding the tradeoffs with respect to accuracy and time required to arrive at the solution for a given value of $\varepsilon$. For this, we first compare the performance of the MConF FPTAS from [Fle99] with the solution obtained by solving the problem optimally for the topology shown in Fig. 4.3 
using the LP solver called LINDO [LINDO]. Fig. 6.7 shows the tradeoff in terms of the percentage difference with respect to the optimal solution and the time taken by the $\varepsilon$-approximation implementation in order to arrive at the solution, which can be observed to exhibit an exponential behaviour. Also, from Fig. 6.8, we see the approximation algorithm asymptotes to the optimal solution with the increasing value $(1-\varepsilon)$.

Considerations such as size of the network should be taken into account while choosing the value of $\varepsilon$. It is expected that the execution time of the $\varepsilon$-approximation algorithm will increase with an increase in size of the network. Another factor that influences the choice of $\varepsilon$ is the time scales at which the events are expected in the context the VSP's implementing the TA service to enable dynamic bandwidth service to the VPNs. In a highly dynamic environment with very high call rates, where resource state of the network changes at smaller time scales, it would be recommended to set $\varepsilon$ to achieve an execution time corresponding to this time scale, while in case of low load conditions the execution time can be a traded off to achieve more accurate solution by choosing lower values of $\varepsilon$. For our network, we chose for simulation analysis an (1-E) value of 0.3 to achieve FPTAS execution time of less than 1 second. 


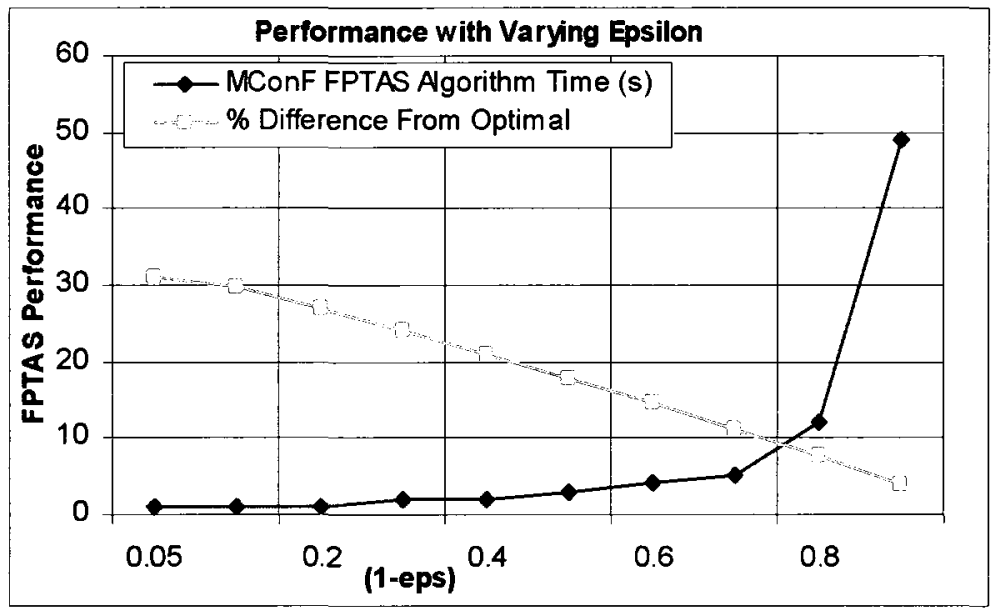

Figure 6-7: Objective 1, Performance of MConF FPTAS with varying approximation factor (Network-1)

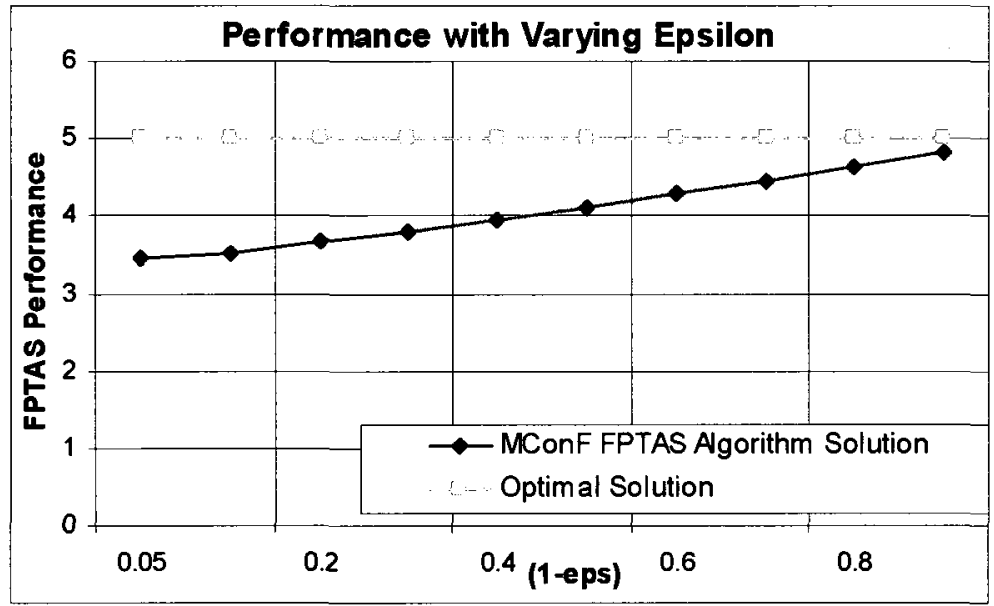

Figure 6-8: Objective 1, Comparing MConF FPTAS and LP optimal solution with varying approximation factor (Network-1)

Objective 2(a): Performance of MConF based partitioning heuristic

Fig 6.9-6.12 shows the performance of MConF based subgraph partitioning scheme for generating VPN TA by varying the mean call holding time $(H)$ and upper bound on the uniformly distributed requested bandwidth $(X)$.

With respect to abstraction efficiency (Fig 6.9), the MConF based algorithm achieved an abstraction efficiency of 55\% at lower load conditions, which decreases by 
$10 \%$ with an increasing load on the network. This is because increasing the mean call holding time of VPN bandwidth requests causes the network utilization to increase, as shown in Fig. 6.9. Hence, the residual capacity for future abstraction reduces, which results in an increasing variance of residual capacity among the links resulting in lesser aggregate commodity flows, causing the resource partitioning scheme to result in lower abstraction efficiency.

With respect to call performance in Fig. 6.10 (i.e increasing $H$ ), we observe that at lower load conditions, the success ratio is close to $100 \%$, but as the load increases, the success ratio decreases. This is because of increasing misscall ratio. It should be noted that crankback ratio is almost 0 for all the load conditions. The good crankback ratio is achieved because of the subgraph partitioning method used for TA generation, but this is at the cost of deteriorating misscall ratio performance. The main reason for misscall ratio to increase with increasing load is due to the reducing abstraction efficiency as noted earlier. With lesser abstraction efficiency, the virtual links of the SSA is associated with lesser virtual link capacity, this makes the VPNs terminate increasing percentage of calls locally, increasing the misscall ratio.

Fig. 6.11 shows the abstraction efficiency performance with respect to varying size of the capacity request $(X)$. Here, the abstraction efficiency is noted to only reduce by $2 \%$ over the range of values of $X$. This can be reasoned by observing the very poor network utilization achieved with increasing value of $X$. We observe that the network utilization increases when $X$ is in the range of $[50,100]$, but decreases beyond that. This is due to the following reason. From the simulation it was observed that, for Network-1, and 
with fair resource partitioning applied for the five VPNs, most of the virtual links of SSA is associated with virtual capacity of around 150 units. This results in VPNs terminating increasing number of calls locally, hence significantly increasing the misscall ratio when $X>100$, this can be observed in Fig. 6.12. This is also the reason for significant decrease in performance of success ratio as shown in Fig. 6.12. Objective 4 proposes a simple technique to overcome this conservative nature of centralized mode of TA generation.

In conclusion, we observed that applying MConF based TA generation approach achieves very good success ratio at low load conditions, but at higher loads the success ratio deteriorates because of poor misscall ratio performance. Also, applying centralized mode of TA generation results in very good crankback ratio performance. With increasing bandwidth request size, the success and misscall ratio performance was noted to be poor because of conservative exposure of bandwidth resources to the VPNs.

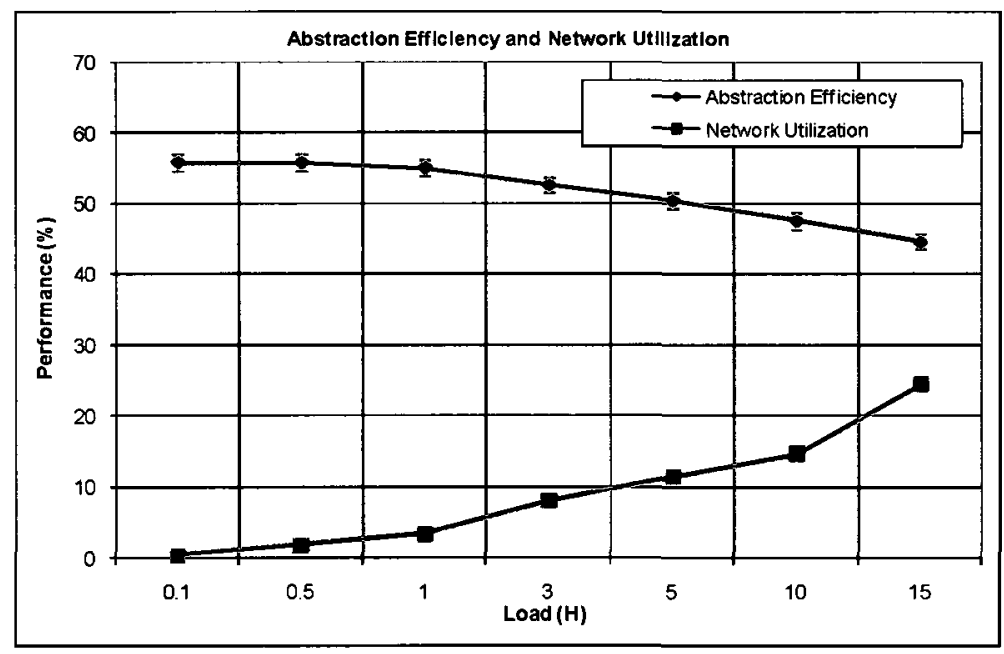

Figure 6-9: Objective 2(a), VSP abstraction efficiency and core network utilization with varying mean holding time $(\mathrm{H})$ for $\mathrm{MConF}$ based abstraction scheme with $95 \%$ C.I (Network-1) 


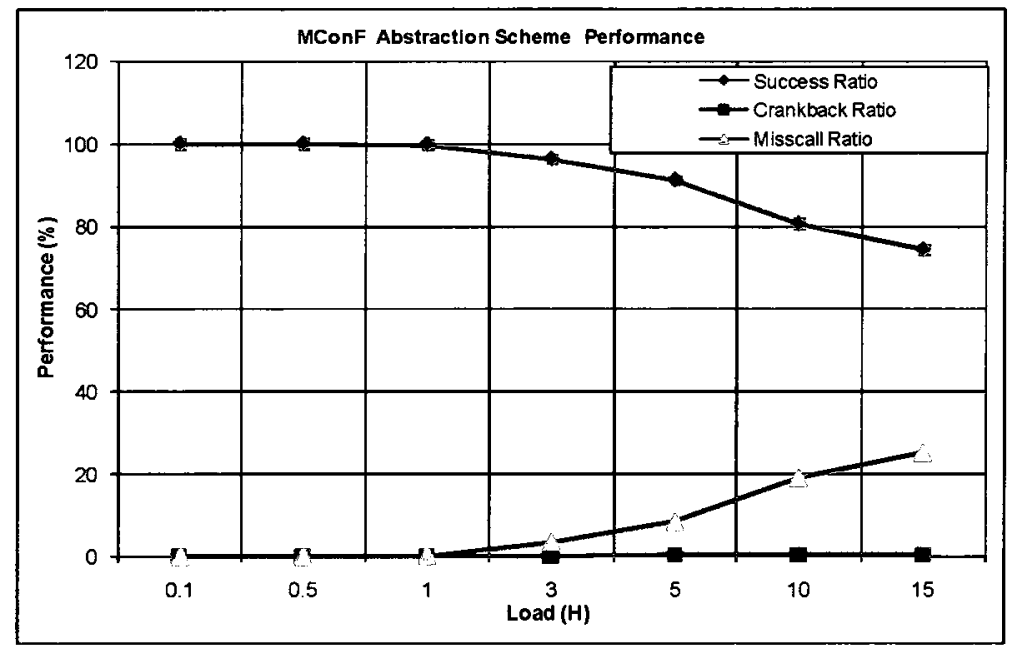

Figure 6-10: Objective 2(a), VPN call performance with varying mean holding time (H) for MConF based abstraction scheme with $95 \%$ C.I (Network-1)

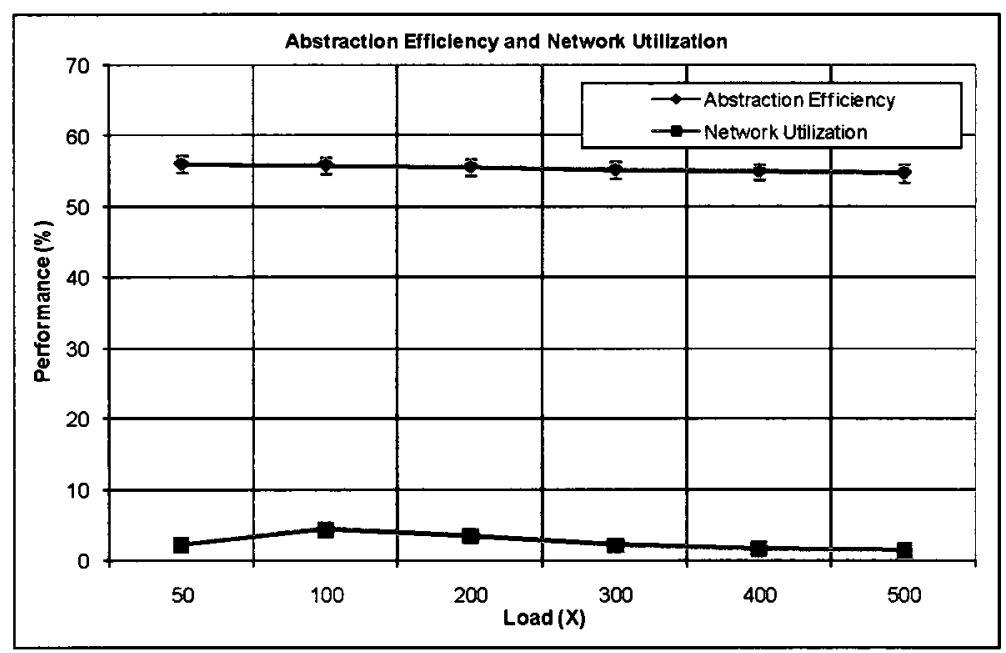

Figure 6-11: Objective 2(a), VSP abstraction efficiency and core network utilization with varying bandwidth request $(X)$ for $M C$ onF based abstraction scheme with $95 \%$ C.I (Network-1) 


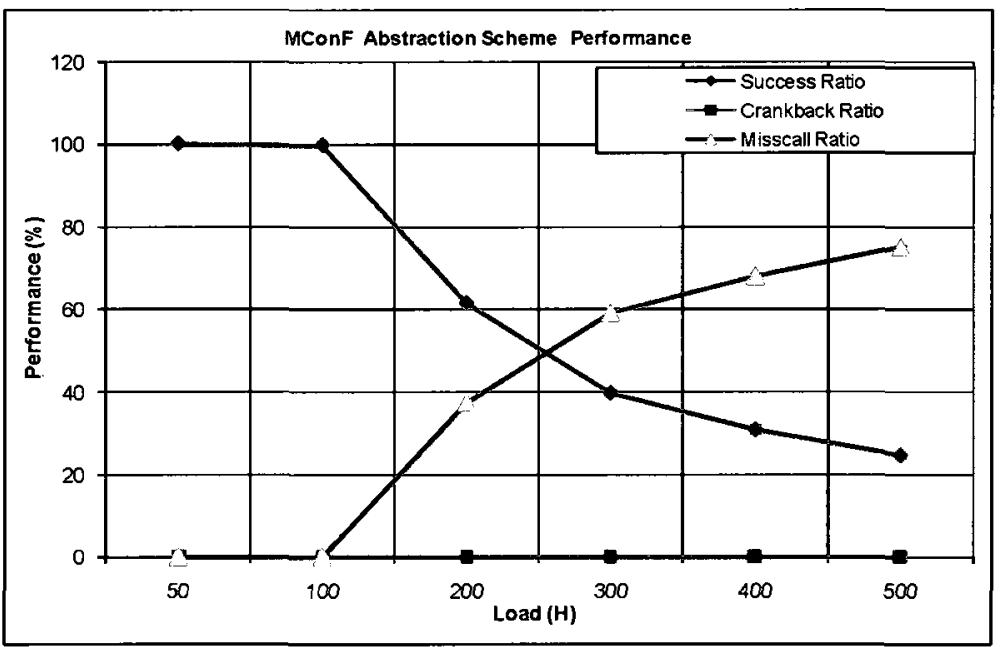

Figure 6-12: Objective 2(a), VPN call performance with varying bandwidth request (X) for MConF based abstraction scheme with 95\% C.I (Network-1)

Objective 2(b): Evaluation of MMCF based partitioning heuristic and comparison with MConF based heuristic

To show the improvement achieved by MMCF over MConF based approximation algorithms, we first discuss the results generated using offline implementation of the MConF and MMCF FPTAS and compare their performance with respect to multiple random graphs of various sizes ranging from 5 to 50 nodes.

Fig. 6.13 compares the average value of $\beta$ or the commodity throughput, i.e the average of the ratio of aggregate commodity flow (using MConF or MMCF) to the maximum flows of the commodities for each graph instance. We observe that because of the flow maximizing objective of MMCF formulation, it achieves about $10 \%-15 \%$ better throughput than MConF algorithm. Also, Fig. 6.14 compares the abstraction efficiency of MConF based heuristic and MMCF heuristic from a simulation run. Recall that abstraction efficiency refers to the ratio of total capacity that result from the partitioning process to the total residual capacity considering the link state of the network during 
computing the ratio. With respect to this metric, we observed that MMCF outperforms MConF by $20 \%$.

Figs. 6.15-6.16 compare the call performance of MConF and MMCF based heuristics under varying load from simulation analysis. From Fig. 6.15, which compares the success ratio, we observe that the partitioning approach using MMCF performs better than MConF by about $5 \%$ particularly at higher load conditions. This is because of the gain achieved by MMCF in terms of abstraction efficiency. A similar observation was also made in the case of crankback and misscall ratio metrics as shown in Fig 6.16. We can see that MMCF based TA scheme performing 5\% better than MConF based TA scheme in terms of this aggregate metric. Fig. 6.17 compares the network utilization achieved by the MConF and MMCF approaches; the better call performance and abstraction efficiency of the MMCF based approach results in better network utilization in the range of $5 \%$ for various load conditions. As expected, a significant difference in the two approaches was noted with respect to fairness of the commodity flows. Though MMCF was observed to achieve high abstraction efficiency, it fared poorly in terms of fairness among the commodity flows. Fig. 6.18 compares the average standard deviation of the commodity flows achieved by the MMCF and MConF based partitioning algorithms. We see that at lower network utilization conditions, the MConF performs fifty times better than MMCF. The decreasing difference of the fairness performance of the two schemes is due to decreasing difference of abstraction efficiency between the two schemes with increasing load as observed in Fig. 6.19. 


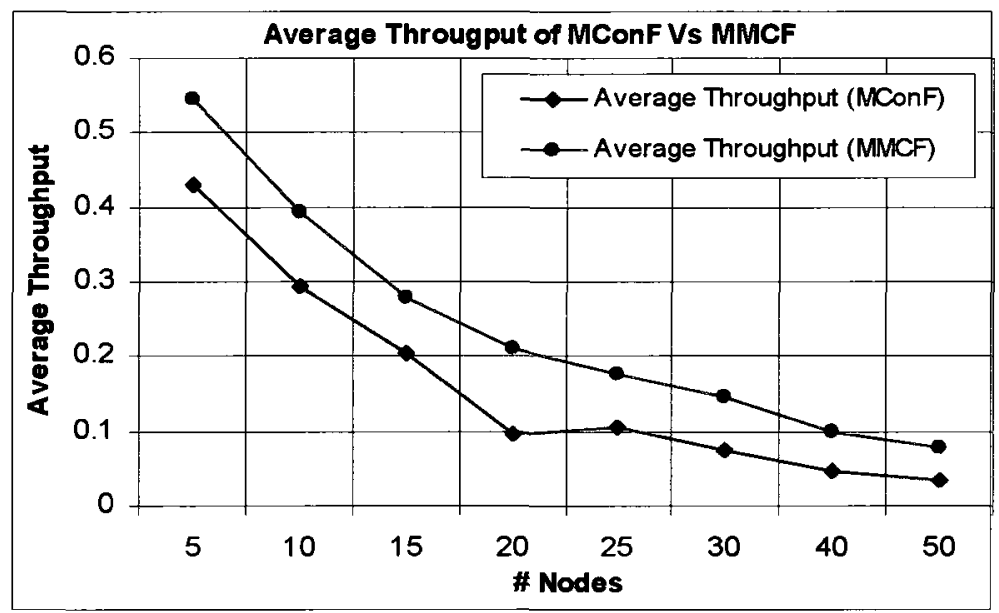

Figure 6-13: Objective 2(b), Comparison between the achieved throughputs of MConF and MMCF FPTAS with varying size of graph

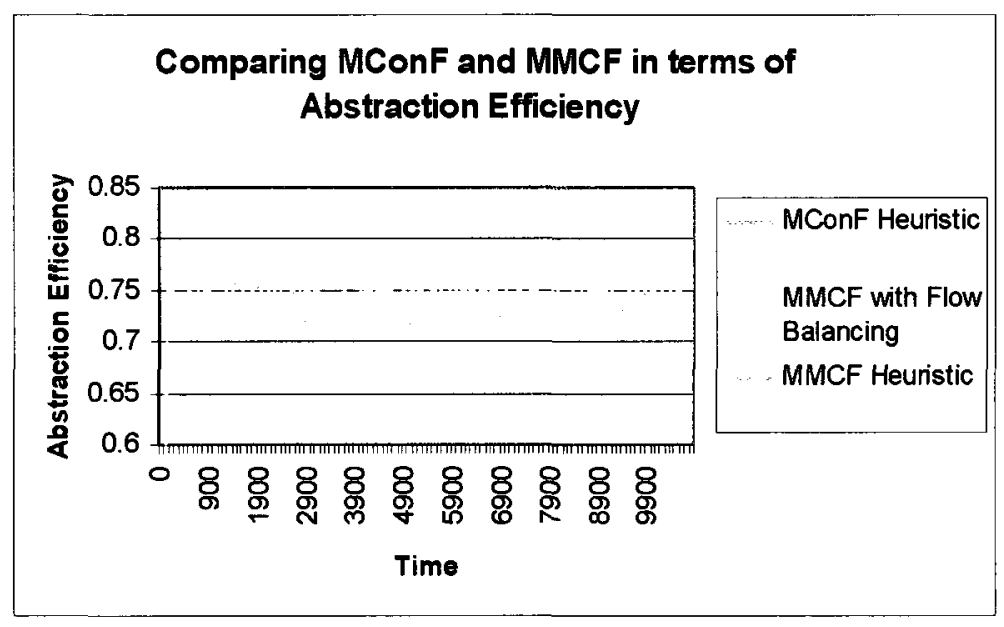

Figure 6-14: Objective 2(b), Throughput comparison of MConF and MMCF based abstraction schemes from a simulation run (Network-1) 


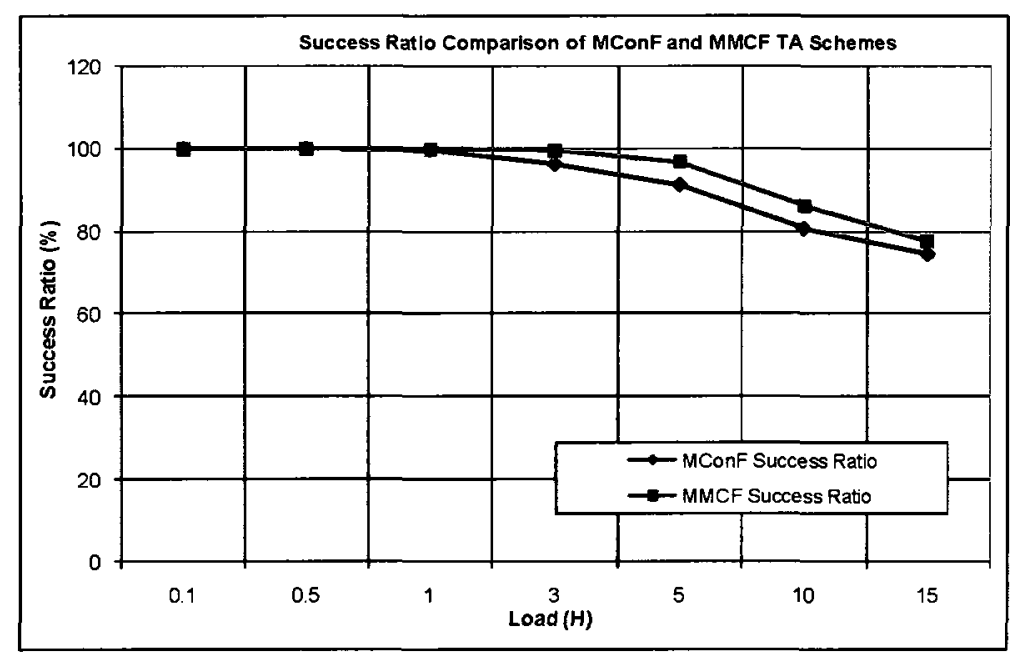

Figure 6-15: Objective 2(b), VPN call performance comparison of success ratio MConF and MMCF based abstraction schemes with varying mean holding time (H) with $95 \%$ C.I (Network-1)

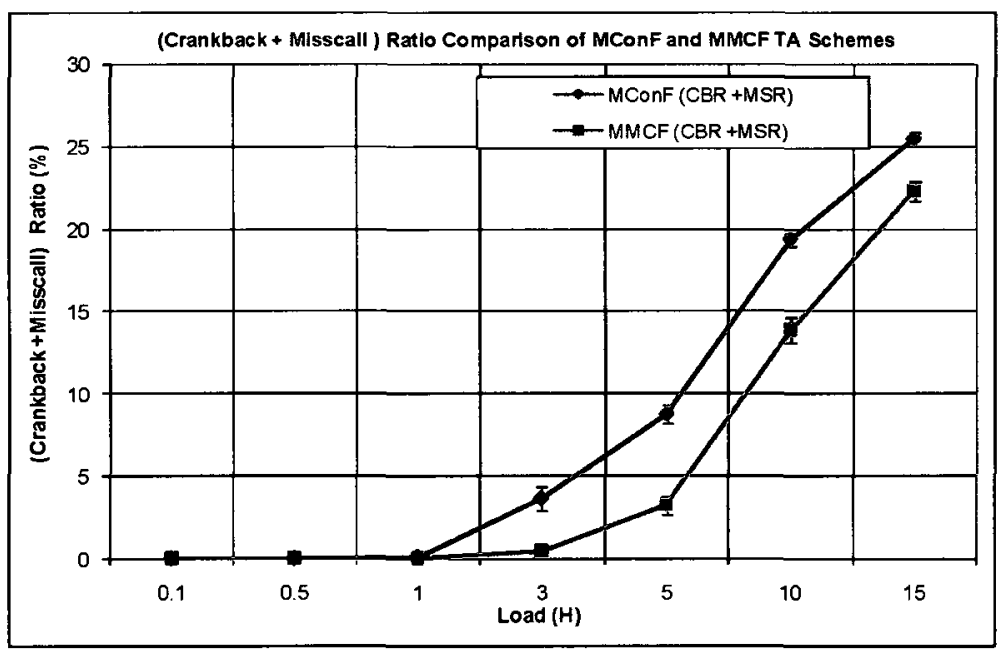

Figure 6-16: Objective 2(b), VPN call performance (crankback + misscall) ratio comparison of MConF and MMCF based abstraction schemes with varying mean holding time (H) with 95\% C.I (Network-1) 


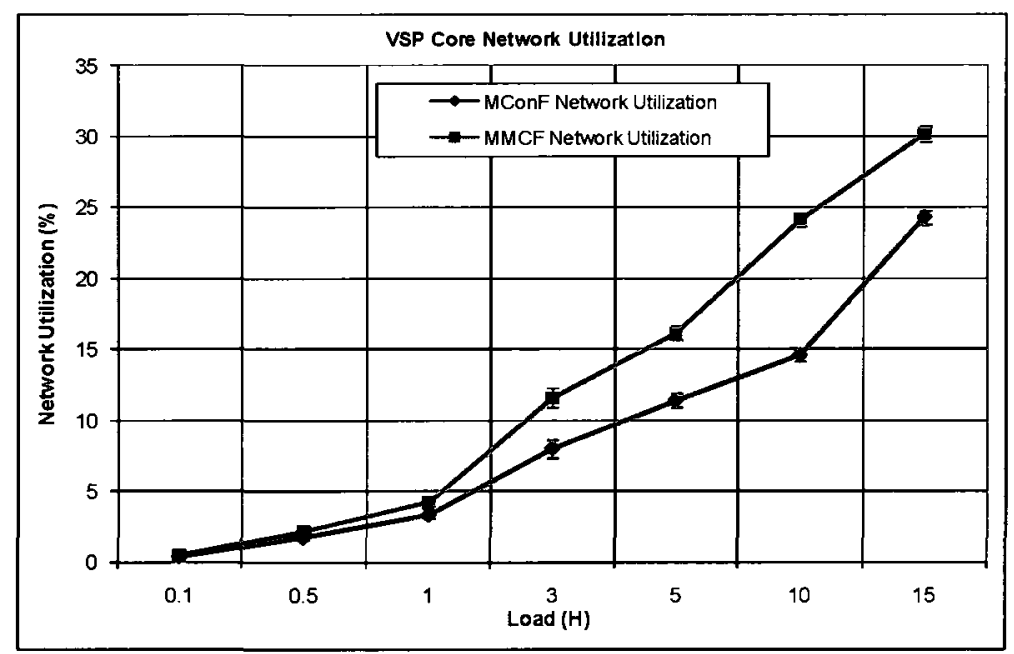

Figure 6-17: Objective 2(b), VSP network utilization comparison of MConF and MMCF based abstraction schemes with varying mean holding time $(\mathrm{H})$ with $95 \%$ C.I (Network-1)

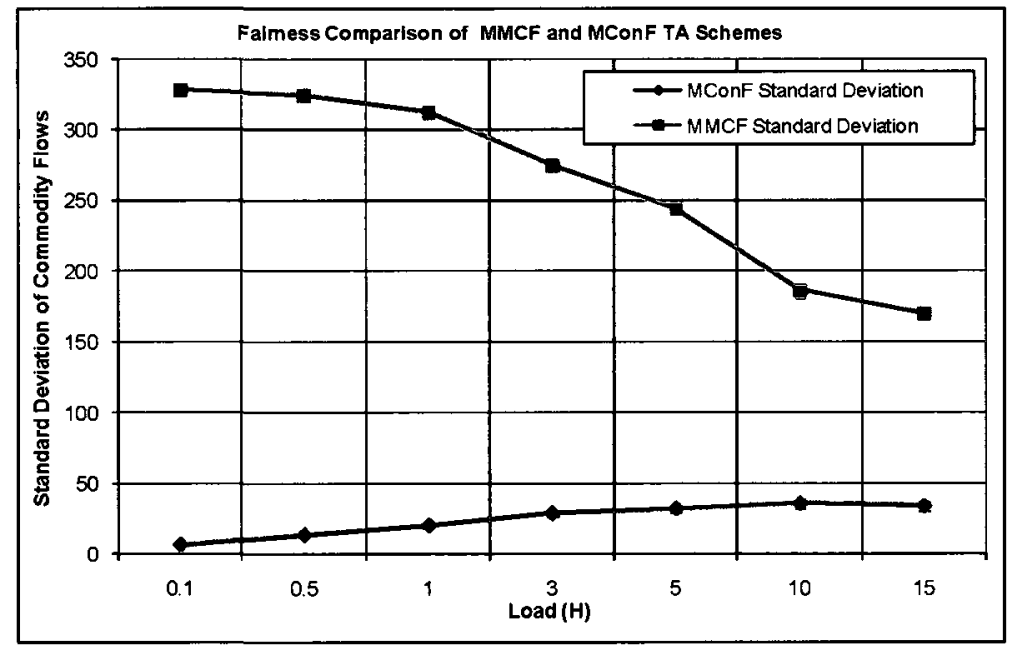

Figure 6-18: Objective 2(b), Comparison of fairness of MConF and MMCF based abstraction schemes with varying mean holding time (H) with $95 \%$ C.I (Network-1) 


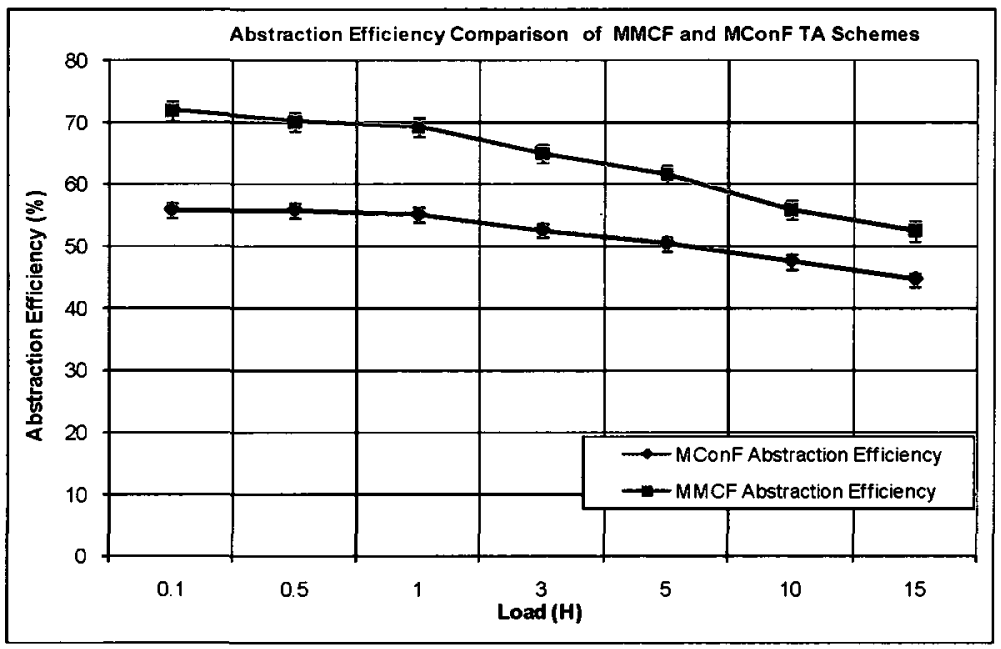

Figure 6-19: Objective 2(b), Comparison of abstraction efficiency of MConF and MMCF based abstraction schemes with varying mean holding time $(H)$ with $95 \%$ C.I (Network-1)

\section{Comparing MConF and MMCF TA scheme performance for Network-2 and}

\section{Network-3}

The performance difference between MConF and MMCF based abstraction schemes for both these network topologies was noted to be similar to that observed for Network-1. The reason for this is primarily because of the performance difference with respect to abstraction efficiency between MMCF and MConF based partitioning schemes, which on average was noted to be around $20 \%$ for both the topologies.

Fig. $6.20(\mathrm{a}-\mathrm{b})$ compares the call performance metrics for Network-2. Fig. 6.21 (a-b) compares the abstraction efficiency and network utilization. MMCF based abstraction scheme was observed to perform 3\% better than MConF based abstraction scheme at higher loads. Similar difference in performance was also noted with respect to misscall ratio. The crankback ratio as in the case of Network- 1 was noted to be almost 0 . 
The network utilization of MMCF based scheme was noted to be $4 \%$ better than MConF based scheme, which also correlates with the performance difference with respect to the success ratio.

Fig. $6.21(a-b)$ compares the call performance for Network-3 and Fig. 6.22 (a-b) compares the performance with respect to abstraction efficiency and network utilization. In this case the performance of MMCF based scheme was observed to be $6 \%$ better than MConF base abstraction scheme. Similar level of performance difference was also noted with respect to misscall ratio and network utilization metrics too.
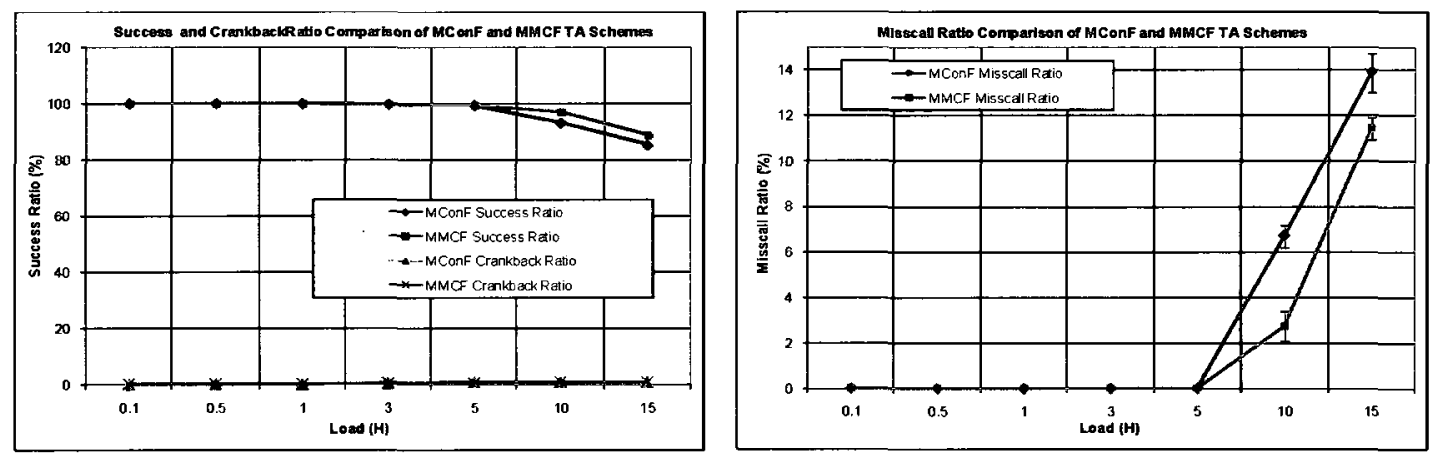

Figure 6-20 (a-b): Objective 2(b), Comparison of success, crankback and misscall ratio of MConF and MMCF based abstraction schemes with varying mean holding time (H) with $95 \%$ C.I (Network-2)
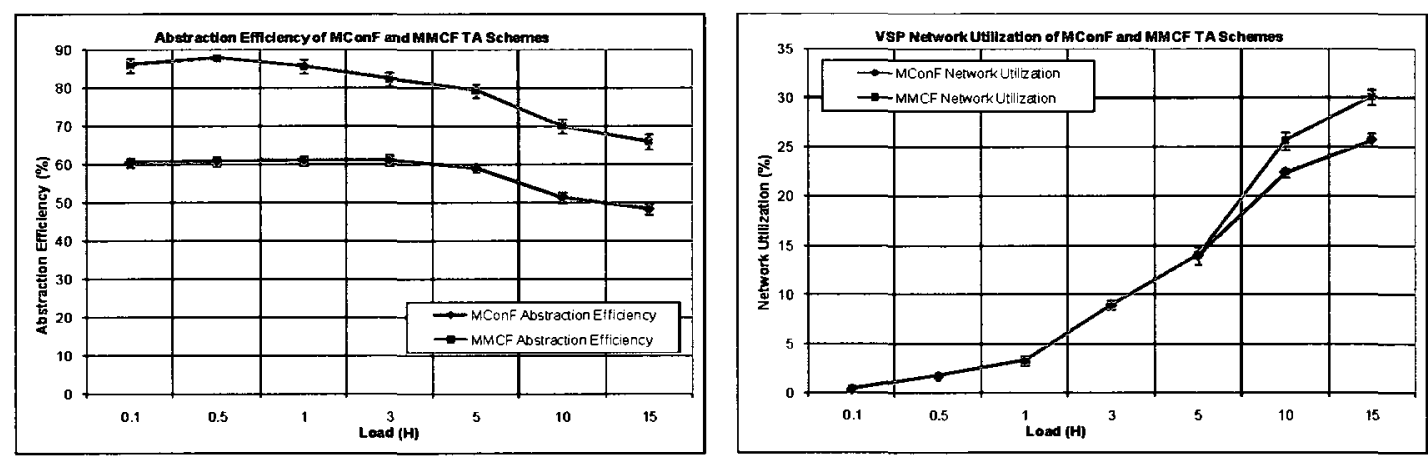

Figure 6-21 (a-b): Objective 2(b), Comparison of abstraction efficiency and network utilization of MConF and MMCF based abstraction schemes with varying mean holding time (H) with 95\% C.I (Network-2) 

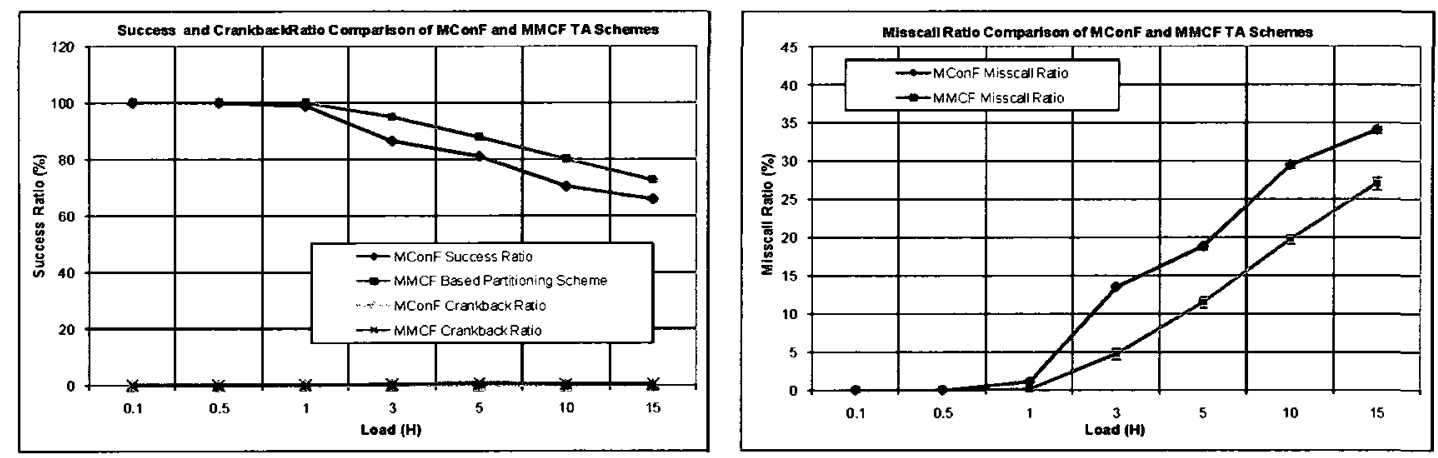

Figure 6-22 (a-b): Objective 2(b), Comparison of success, crankback and misscall ratio of $M C O n F$ and $M M C F$ based abstraction schemes with varying mean holding time (H) with $95 \%$ C.I (Network-3)
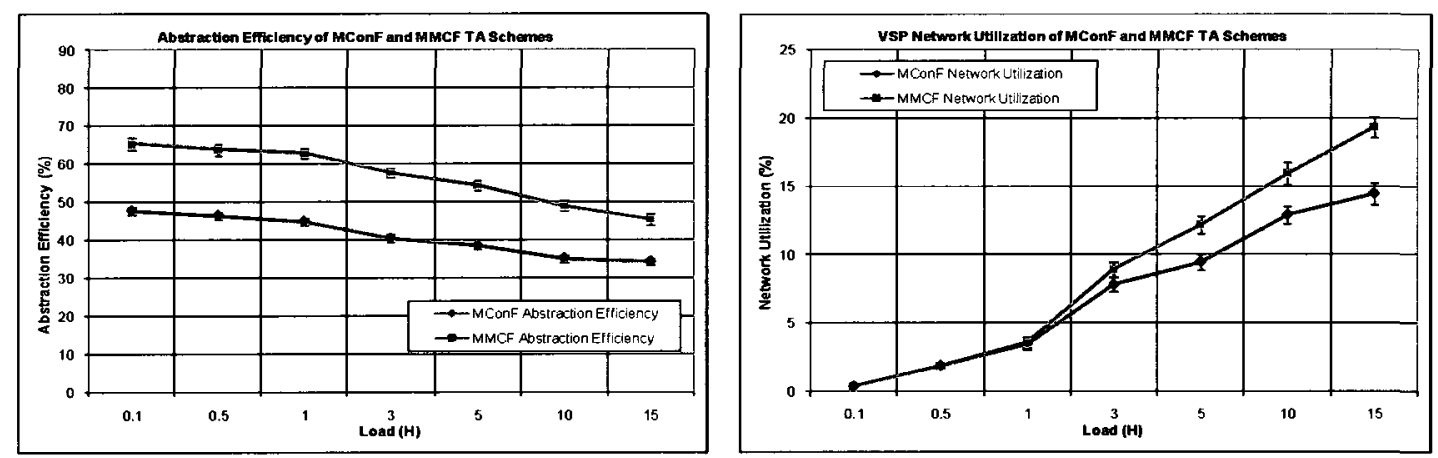

Figure 6-23: Objective 2(b), Comparison of abstraction efficiency and network utilization of MConF and MMCF based abstraction schemes with varying mean holding time (H) with 95\% C.I (Network-2)

As observed in Objective 2, the performance results from the simulation study for the remaining objectives was observed to be similar for the three network topologies. For this reason we limit our discussion of the remaining objectives (for those related to with simulation analysis) with respect to Network-1. For Networks $2 \& 3$ we summarize their results for each objective by referring to the graphs presented in Appendix A. 


\section{Objective 3(a): Offline performance comparison of MMCF formulations and} improvements to achieve fairness

Here, we study and compare the performance of MMCF formulation and its variations to address the fairness issue in an offline manner. Before discussing the results, we wish to note that by the very nature of the objective function, MMCF will always result in the maximum abstraction efficiency and least fairness, and as stated earlier, MConF will result in commodity flows with maximum fairness, while achieving the least abstraction efficiency. The performance of the MMCF variations MB-1 and MB-2 is expected to lie within these extremes. The goal of this objective is to understand which formulation strikes the best balance in achieving abstraction efficiency matching that of the MMCF scheme and fairness matching that of the MConF scheme.

We study the performance of these formulations by running over well known provider networks referred from [Ho07], and we characterize their performance in terms of two metrics: the aggregate flow achieved by the formulation from which abstraction efficiency is obtained and the fairness of the commodity flows among the commodities. The fairness is characterized in terms of the standard deviation among the commodity flow.

We discuss the performance results with reference to graphs in Fig. 6.24 and Fig. 6.25. In Fig. 6.24, we see that MMCF achieves the best abstraction efficiency but also suffers in terms of faimess as shown in Fig. 6.25, which results in maximum standard deviation among the commodity flows. On the other hand, the MConF resource partitioning scheme achieves the least abstraction efficiency, while achieving the least standard deviation and maximum fairness. Comparing the two MMCF variations, variation 
MB-1, which defines its flow bounds using the maximum flow and MMCF flow, achieves abstraction efficiency close to that of MMCF formulation but makes only a small gain in addressing the fairness issue. The second variation MB-2 that uses the MConF and MMCF outputs to define the bounds results in as much or slightly less aggregate flow when compared to MMCF or MB-1 formulation, while achieving maximum fairness of the three MMCF formulations. Hence we see that, comparing the two bounded MMCF formulations, the MB-2 variation that uses the MConF flows to define the bounds over the commodity flows, performs the best in terms of achieving MMCF's abstraction efficiency, while also improving the fairness among the commodity flows.

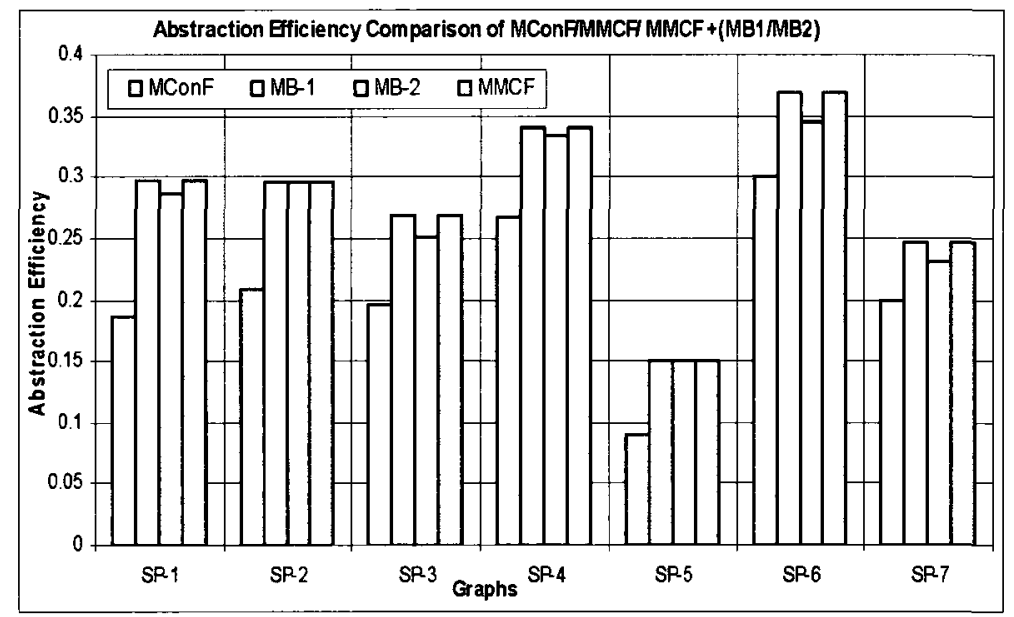

Figure 6-24: Objective 3(a), Offline comparison of abstraction efficiency of MConF, MMCF, MMCF with MB1/MB2 formulations for different graphs 


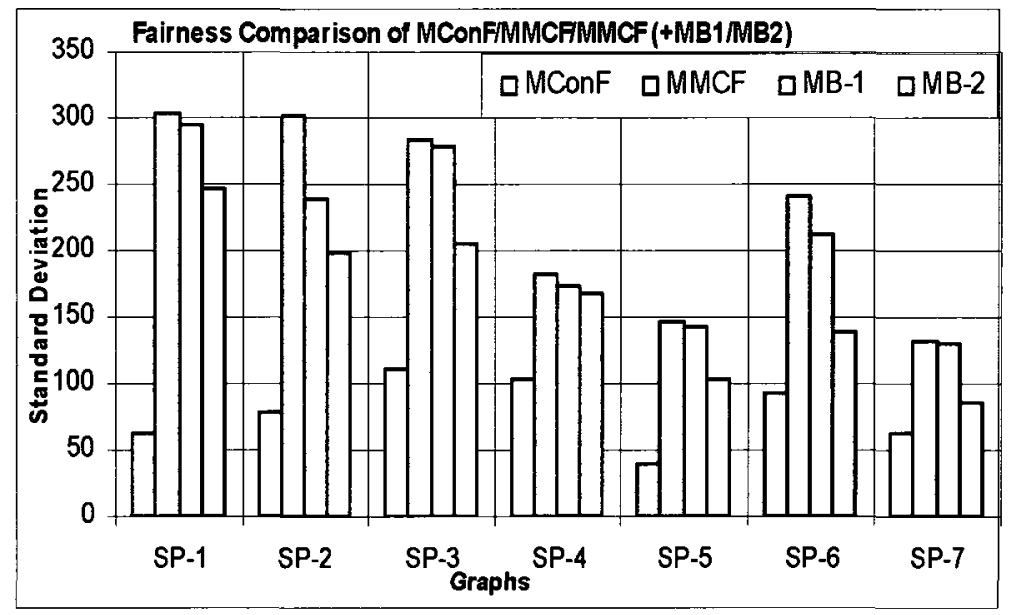

Figure 6-25: Objective 3(a), Offline comparison of fairness of MConF, MMCF, MMCF with MB1/MB2 formulations for different graphs

Objective 3(b): Comparing performance of MMCF based partitioning scheme and MMCF augmented with flow balancing heuristic

For this objective, we first present an offline comparison of the commodity throughput of the MMCF based algorithm with and without enabling flow balancing. The offline analysis used the FPTAS with $\varepsilon$ set to 0.5 . The fair balancing algorithm was applied on the commodity flows obtained by solving MMCF using FPTAS. For the offline analysis, we used the same graphs [Ho07] that were used in the study of Objective 2. Fig 6.26 compares the variance of the commodity throughputs before and after flow balancing (FB) was applied. After applying the flow balancing scheme, we noticed an average reduction in the variance of about $9 \%$ among the commodity flow throughputs. This demonstrates the usefulness of applying flow balancing heuristic so as to improve fairness of the commodity flow solution obtained using MMCF. 
Figs. 6.27-6.28 compare the performance of abstraction efficiency and success ratio of MMCF with and without the application of the flow balancing heuristic using simulation. With respect both these metrics, the performance of the two scenarios was almost the same. No difference in performance was also noted in terms of other call performance metrics too. This is expected since the fair partitioning algorithm only improves the fairness of the commodity flows obtained from MMCF based heuristic, which is done by re-balancing the flows without affecting the abstraction efficiency.

As expected, an important difference was noted with respect to fairness, as seen in Fig. 6.29. With flow balancing enabled over MMCF commodity flows, we would expect the variance of the commodity throughput factor to reduce. The improvement in terms of standard deviation when flow balancing is applied was noted to be about $25 \%$ better; this demonstrates the usefulness of the flow balancing heuristic. From the Fig. 6.29, we observe that with increasing load the difference between the variance of the commodity flows of MMCF and MMCF with flow balancing decreases. This is because, the increasing network load results in proportional increase in network utilization, hence decrease in abstraction efficiency. This results in commodities to achieving lesser aggregate MMCF flows, thereby decreasing the variance between aggregate flows of the commodities. This causes flow balancing scheme to achieve lesser improvement in terms of faimess with increasing load. 


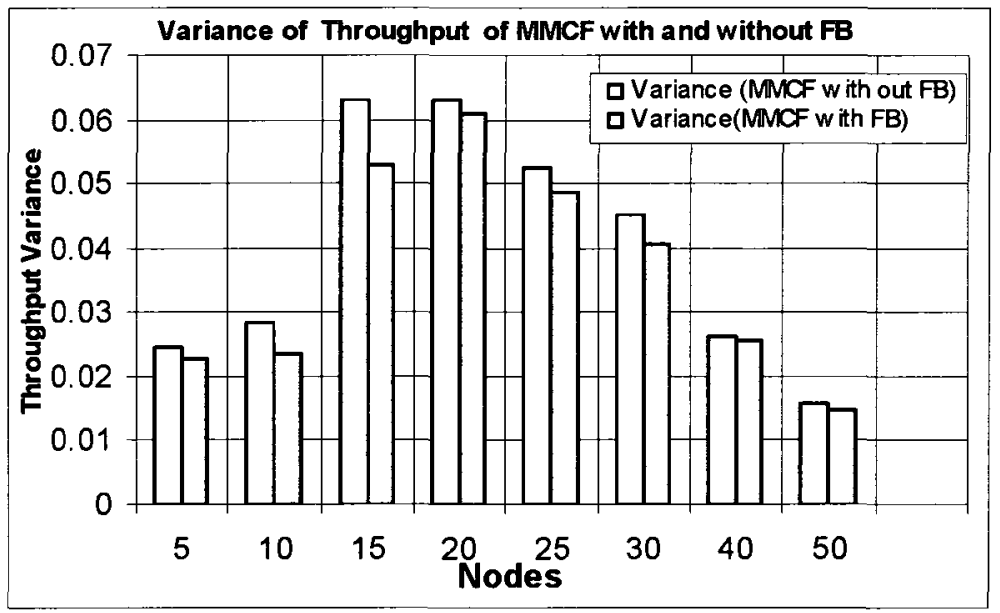

Figure 6-26: Objective 3(b), Comparing variance of commodity throughputs of MMCF with and without Fair Balancing for different graphs

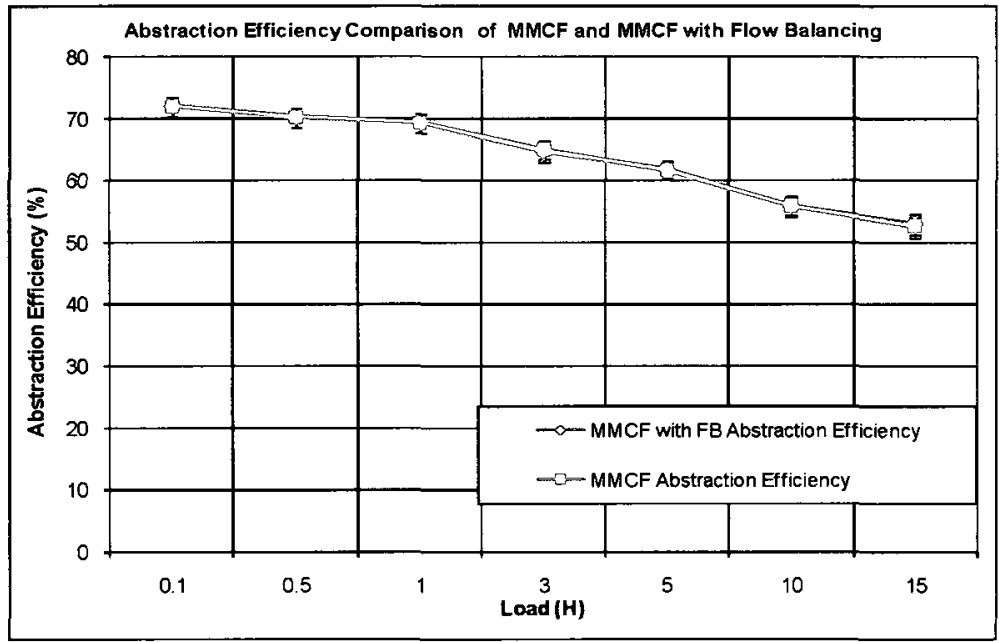

Figure 6-27: Objective 3(b), Comparing VSP abstraction efficiency of MMCF with and without Fair Balancing with varying mean holding time (H) with $95 \%$ C.I (Network-1) 


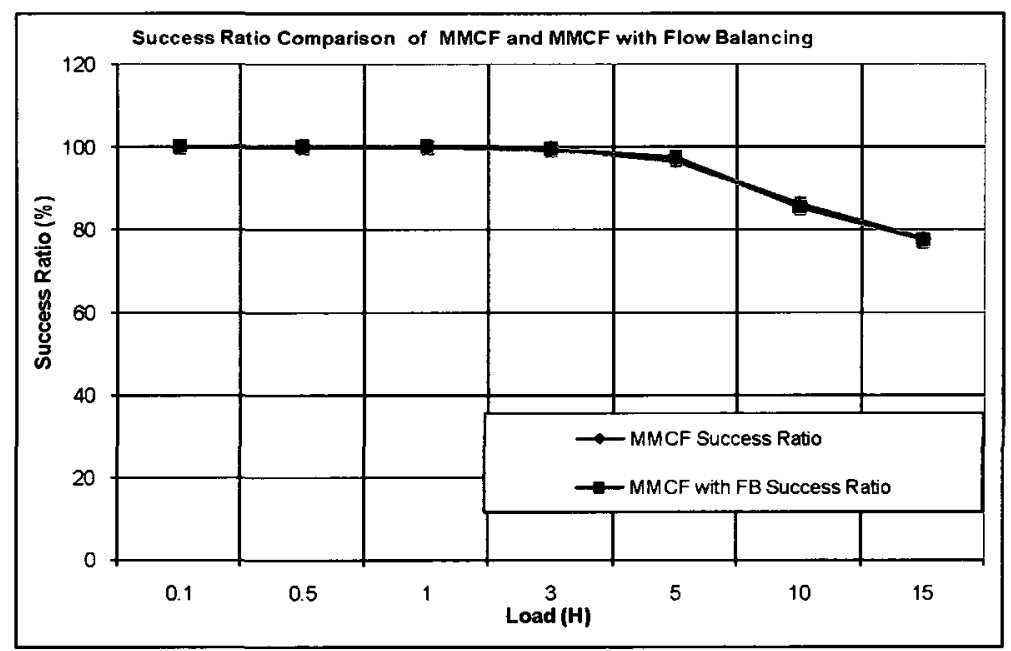

Figure 6-28: Objective 3(b), VPN call performance comparing success ratio of MMCF with and without Fair Balancing with varying mean holding time (H) with 95\% C.I (Network-1)

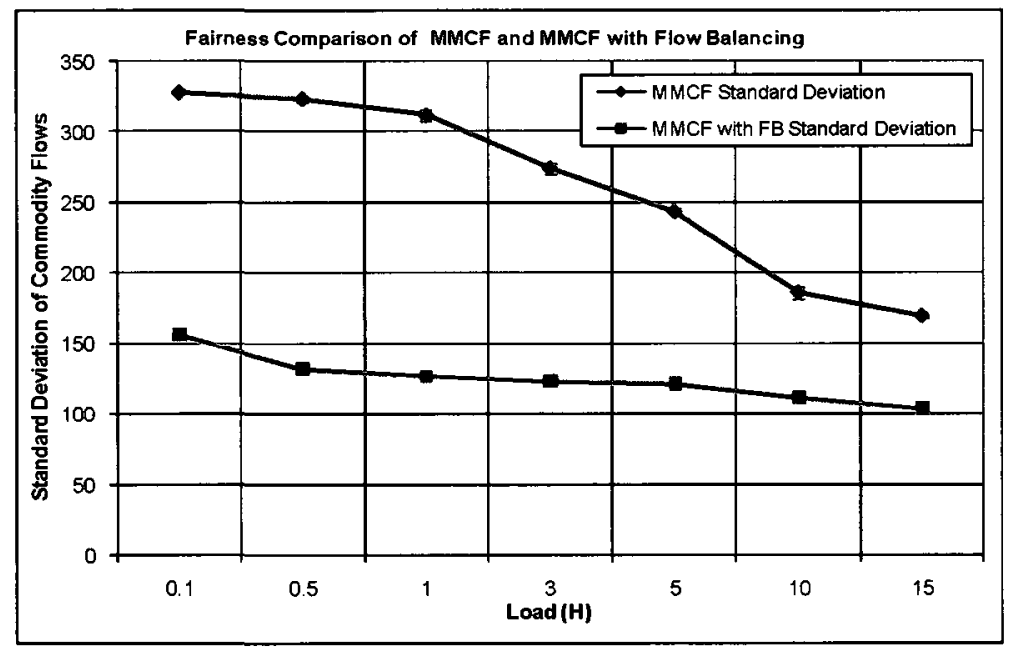

Figure 6-29: Objective 3(b), Comparing fairness of MMCF with and without Fair Balancing with varying mean holding time $(\mathrm{H})$ with $95 \%$ C.I (Network-1) 
Comparing performance of MMCF and MMCF with flow balancing scheme for Network-2 and Network-3

Fig. A-1(a-b) compares the abstraction efficiency and success ratio of MMCF and MMCF with flow balancing for Network-2. Fig. A-2 compares the commodity fairness for the two cases. Similar to what was noted and discussed in the case of Network-1, the abstraction efficiency and success ratio didn't show any difference in performance. However as expected, the standard deviation of the aggregate commodity flows with flow balancing improves on an average by $24 \%$ compared to the case when flow balancing is not applied. The reasons for decreasing difference in improvement of the commodity flows with increasing load are similar to that discussed in the case of Network-1.

Fig. A-3(a-b) compares the abstraction efficiency and success ratio of MMCF and MMCF with flow balancing for Network-3. Fig. A-4 compares the standard deviation of the MMCF commodity flows for the two cases. In this case while the abstraction efficiency and success ratio didn't show any difference in performance, the standard deviation of the aggregate commodity flows achieved by MMCF improves on an average by $20 \%$ after flow balancing is applied.

From the above observations for the three network topologies, we can conclude that flow balancing heuristic can be successfully applied to improve the fairness issue of the MMCF based TA scheme. 


\section{Objective 4(a): Comparing Performance of centralized abstraction and} decentralized abstraction schemes

In this objective, we compare the difference in performance of the two modes of TA generation in terms of VPN call performance and network utilization. From the three decentralized schemes proposed in the previous chapter, we choose the maximum capacity scheme for the analysis. The call performance statistics for the TA gneration using the maximum capacity scheme was collected using the same simulation setting as shown in Table 6.1 for objective 4(a).

Figs. 6.30-6.33 compare the call performance and network utilization of the maximum capacity scheme, MConF, and MMCF based abstraction schemes. As regards to the VPN success ratio in Fig. 6.30, we note that the success ratio of the maximum capacity scheme is better than that of MConF by $10 \%$ and by $5 \%$ with respect to the MMCF based abstraction scheme. With respect to crankback ratio, in Fig. 6.31, we note that maximum capacity scheme's performance degrades by $7 \%$ at maximum load while MConF and MMCF abstraction schemes achieve crankback ratio of less than $1 \%$, which was also noted earlier objectives 1-2. The significant gain in crankback ratio is achieved at the cost of high misscall ratio of $25 \%$ and $22 \%$ for MConF and MMCF based schemes compared to $7 \%$ for maximum capacity scheme. This performance difference is shown in Fig. 6.32. The performance difference in success ratio also translates into good network utilization of the maximum capacity scheme, which performs $10 \%$ and $5 \%$ better than MConF and MMCF based abstraction schemes, this can be observed in Fig. 6.33. 
This performance difference between the decentralized and centralized schemes suggests that minimizing the oversubscription alone, which was the main motivation for developing the centralized schemes, does not translate into good VPN call performance. This is particularly obvious at higher loads when the contention of resources among the VPNs is high. In this case the aggressive nature of the maximum capacity scheme is noted to improve statistical multiplexing of core resources among the VPN banwidth requests, which contibutes to better call performance. The centralized abstraction schemes, because of their conservative exposure of available capacity (derived from logical partitioning schemes), result in poorer misscall ratio performance because of its property of minimizing oversubscription, which results in limited multiplexing of available capacity.

In the next objective we try to improve the misscall ratio performance and in turn the success ratio performance of the centralized scheme by applying the technique of oversubscription of residual link capacity. 


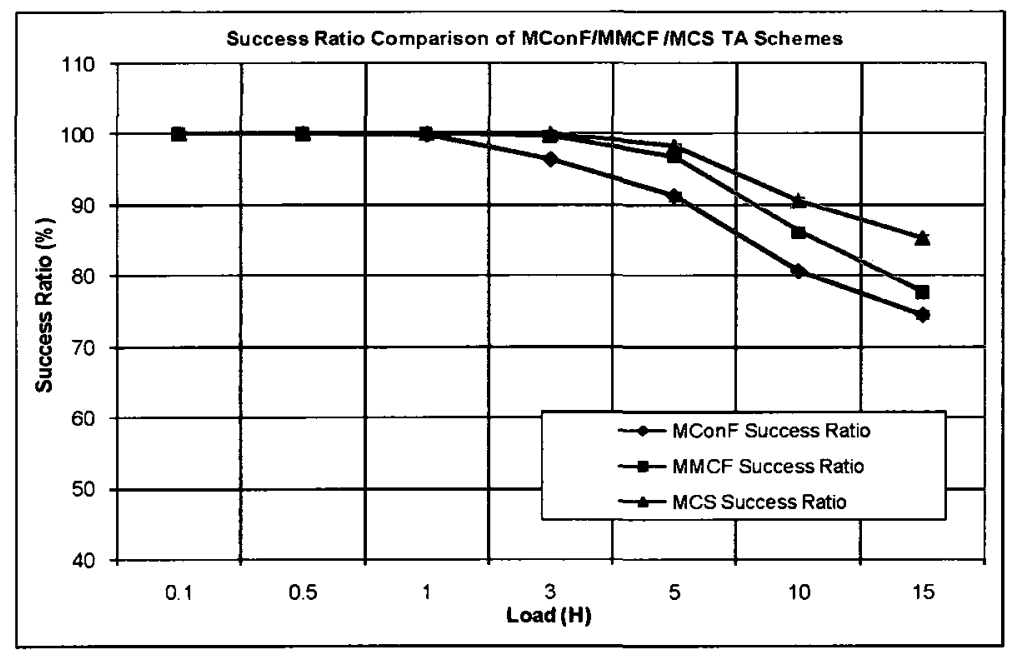

Figure 6-30: Objective 4(a), VPN call performance comparing success ratio of maximum capacity, MConF and MMCF based TA schemes with varying mean holding time (H) with 95\% C.I (Network-1)

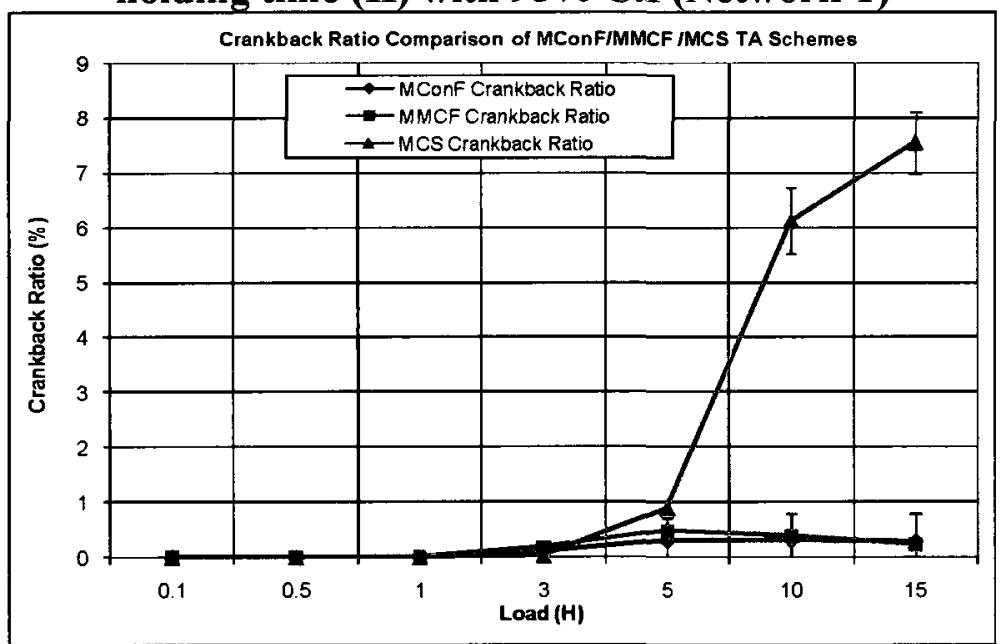

Figure 6-31: Objective 4(a), VPN call performance comparing crankback ratio of maximum capacity, MConF and MMCF based abstraction schemes with varying mean holding time (H) with $95 \%$ C.I (Network-1) 


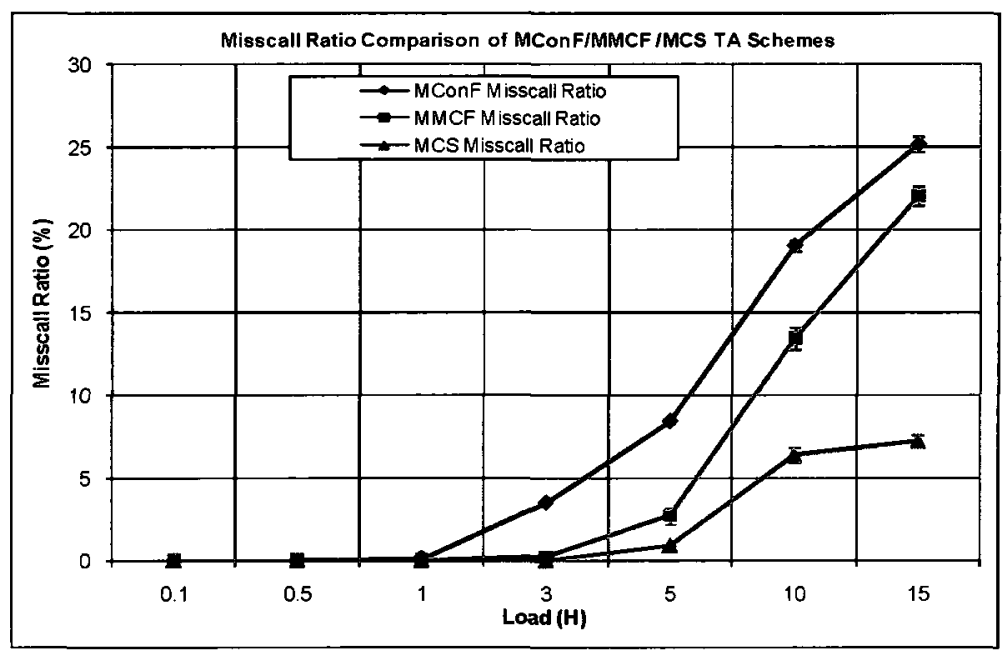

Figure 6-32: Objective 4(a), VPN call performance comparing misscall ratio of maximum capacity, MConF and MMCF based TA schemes with varying mean holding time (H) with $95 \%$ C.I (Network-1)

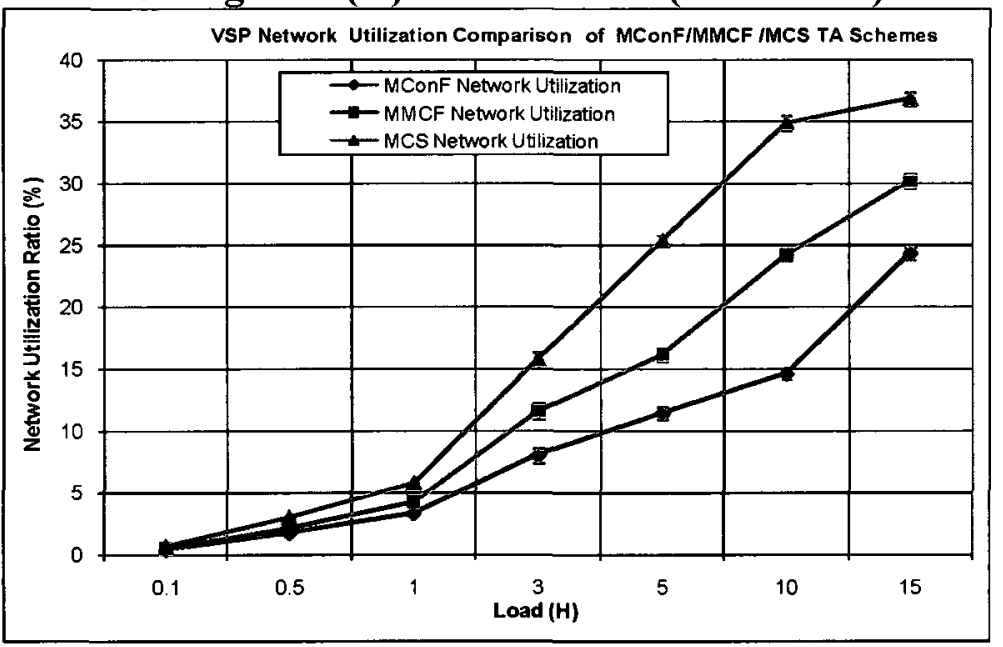

Figure 6-33: Objective 4(a), VSP network utilization comparison of maximum capacity, MConF and MMCF based TA schemes with varying mean holding time (H) with 95\% C.I (Network-1)

Comparing the performance of MConF, MMCF and maximum capacity scheme for Network-2 and Network-3

Fig. A-5(a-b) compares the success and crankback ratio of the centralized and the maximum capacity scheme for Network-2. Fig. A-6(a-b) compares the misscall and 
network utilization for the two cases. Compared to Network-1, in case of Network-2 the difference in performance between the maximum capacity and the centralized scheme is only obvious at higher load conditions. This can be attributed to the smaller topology size of Network-2, which results in lesser network capacity and path availability between any pair of border nodes. This causes the aggressive maximum capacity scheme to not have any advantage over the conservative schemes for low and medium load conditions. This shows that the centralized schemes could perform as good as the decentralized scheme for smaller network topologies. In terms of success ratio, at higher loads, maximum capacity scheme performs $5 \%$ and $7 \%$ better than MConF and MMCF schemes respectively. This is because of better performance of maximum capacity scheme in terms of misscall ratio. While, the centralized schemes performs better than the maximum capacity scheme in terms of crankback ratio, which for the centralized case is observed to be less than $1 \%$, compared to $4 \%$ for maximum capacity scheme at maximum load. Also, the network utilization in the case of maximum capacity scheme is better than that of the centralized schemes by $5 \%$, in line with difference in performance with respect to success ratio.

Fig. A-7(a-b) compares the success and crankback ratio of the maximum capacity, MConF and MMCF schemes for Network-3. Fig. A-8(a-b) compares the misscall ratio and VSP network utilization for Network-3. In this case, the difference in performance between the maximum capacity scheme, and the centralized schemes is wider than that noted in the case of Network-2. The maximum capacity scheme due to its aggressive nature is noted to perform $15 \%$ better than the MMCF scheme and $23 \%$ better than 
MConF scheme at maximum load. The increased difference in performance is also noted with respect to crankback ratio performance. The crankback ratio of the maximum capacity scheme is noted to be $7 \%$ compared to $1 \%$ for the centralized scheme. Correlating with difference in terms of success ratio performance, the maximum capacity scheme results in $15 \%$ better network utilization than the MMCF scheme and $20 \%$ compared to the MConF scheme.

\section{Objective 4(b): Improving the performance of centralized TA schemes by}

\section{oversubcription of link capacity}

One of the observations from the study of the previous objective is that oversubscrition in the context of the TA service in a dynamic bandwidth scenario could help improve statistical multiplexing. In this objective, we study if this notion can be used to improve the conservative nature of the centralized schemes, particularly the misscall ratio performance. We do this by oversubscribing the links of the core network by multiplying the residual capacity of the link by a constant factor $(Y)$ before executing either MConF or MMCF based logical partitioning scheme in Step 1 in Fig. 6.1. Figs. 6.34-6.38 compare the performance of the MConF and MMCF based schemes for a range of values of oversubscription factor $Y$ by varying it in the range of $[1,10]$, the performance statistics are collected for low, medium and high load conditions set to $0.5,1$ and 5 Erlangs. The bandwidth request size $X$ is set to 500 for all the three scenario. This value of $X$ is chosen by observing Fig. 6.12 , where this bandwidth request setting leads to very poor performance in terms of success ratio, which was observed to be around $25 \%$. 
Fig. 6.34 compares the success ratio with varying values of oversubscription factor for differnt load conditions for MConF based TA scheme. At low load of 0.5 Erlang, we can observe that even at $Y$ set to 3, the success ratio improves by $50 \%$. Also the gain in success ratio is observed to improve with increasing value of $Y$. In Fig. 6.36, we see a similar improvement with respect to misscall ratio performance. From Figs. 6.34-6.36 we also observe that, the gain in success and misscall ratio saturates after a particular value of $Y$. For the low load case this value of $Y$ is 7 . The reason for saturation of the success and misscall ratio performance with increasing oversubscription of the link is due to increase in the crankback ratio, in Fig. 6.37, which achieves its maximum value of $3 \%$ when $Y \geq 7$ for the low load case.

With respect to network utilization, in Fig. 6.38, we observe that gain in success ratio also translates into increasing gain in network utilization. The saturation in network utilization is due to the saturation observed with respect to the success ratio metric discussed earlier. Similar trends in gain in success and misscall ratios is observed for network utilization performance for medium and high load cases too.

From Fig. 6.34 we observe that, with increasing load, the overall gain in success ratio from applying oversubscription decreases. That is, the maximum success ratio achieved with the load offered at 0.5 is $97 \%$ compared to $74 \%$ when load is 5 . This is due to two reasons. First, this is due to decrease in abstraction efficiency of MConF based partitioning scheme with increasing load as discussed in objective 1 with reference to Fig. 6.9. Second, with increasing load and increasing network utilization, the resource 
contention among VPNs also increases. This causes the crankback ratio to increase, and this increases with increasing load whose trend can be observed in Fig. 6.37.

Fig. 6.35 compares the gain in success ratio achieved for MMCF based abstraction scheme. The trend in performance with increasing oversubscription factor in terms of success ratio in the case of MMCF based abstraction scheme is similar to that observed for MConF based scheme. The important observation here is that the difference in performance between MMCF and MConF based abstraction scheme reduces with increasing link oversubscription. This is because, with increasing oversubscription the advantage gained by better abstraction efficiency of MMCF reduces, hence MConF begins to perform as good as MMCF at higher oversubscription factors. The reason for decreasing trend of the success ratio performance with increasing load for the MMCF case, is the same as those for the MConF case.

Next we compare the performance of maximum capacity scheme with MConF and MMCF based TA schemes after link oversubscription is applied. For this scenario, from the previous results we choose an oversubscription factor of 9 .

Fig. 6.39 compares the success ratio for the three schemes. Here we observe that MConF and MMCF perform better than maximum capacity scheme particularly at low and medium load conditions. We see MConF and MMCF performing on an average 4\% better than maximum capacity scheme. We also observe that, the difference between performance of MConF and MMCF itself is not significant. This is because, the link oversubscription overshadows the gain in abstraction efficiency achieved by MMCF which was discussed earlier. At high loads, we see the maximum capacity scheme 
performing better than MConF and MMCF based TA scheme. This is because at high loads the crankback and misscall ratio, as shown in Fig. 6.40, deteriorates for MConF and MMCF based schemes, resulting in poorer success ratio than maximum capacity scheme. This shows that oversubscription may not always act favourably in all load conditions. This is so because link oversubscription at high load conditions results in abstractions that are too aggressive, causing VPNs to make poor decision resulting in high crankback and misscall ratio. In such situations the VSP could adapt the technique of using a tuneable oversubscription factor that varies with utilization of network resources.

To summarize the above observations we note that, oversubcribing the core links by a fixed factor does help to overcome the conservative nature of centralized abstraction schemes to a significant extent. Oversubsciption resulted in improved statistical multiplexing of the core resources causing improvement in the VPN call performance and core network utilization metrics for the centralized abstraction schemes. But the gain achieved in terms of success and misscall ratios is also at a cost of increasing crankback ratio. The increasing crankback ratio causes the success ratio to saturate beyond a certain value of $Y$. Further we also showed that with careful selection of $Y$, the centralized schemes can also perform better than the decentralized abstraction schemes. 


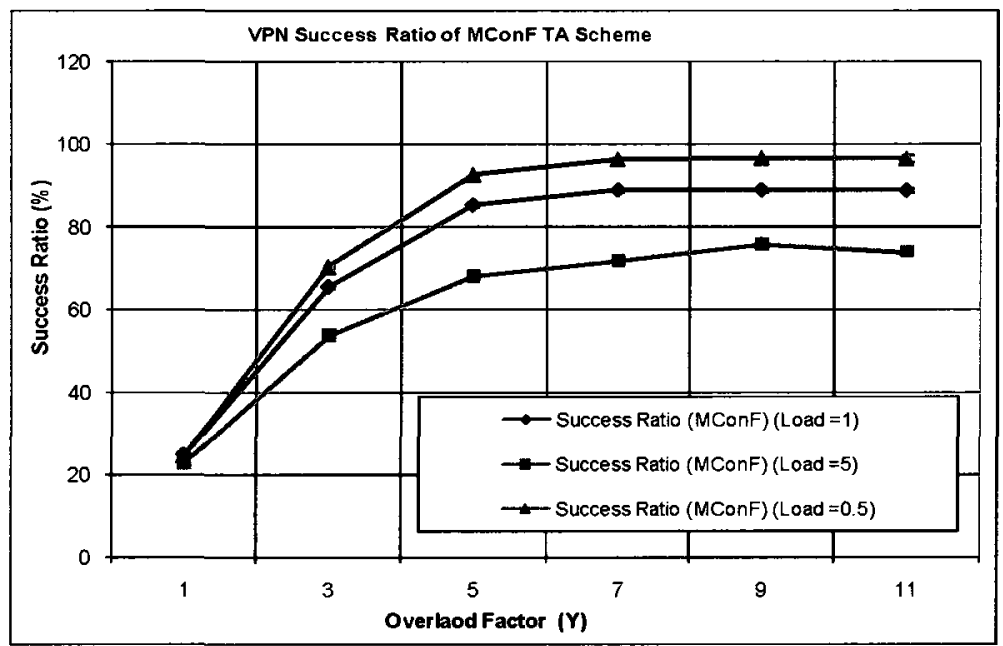

Figure 6-34: Objective 4(b), VPN call performance comparing success ratio of MConF based abstraction scheme with varying oversubscription factor ( $Y$ ) with 95\% C.I (Network-1)

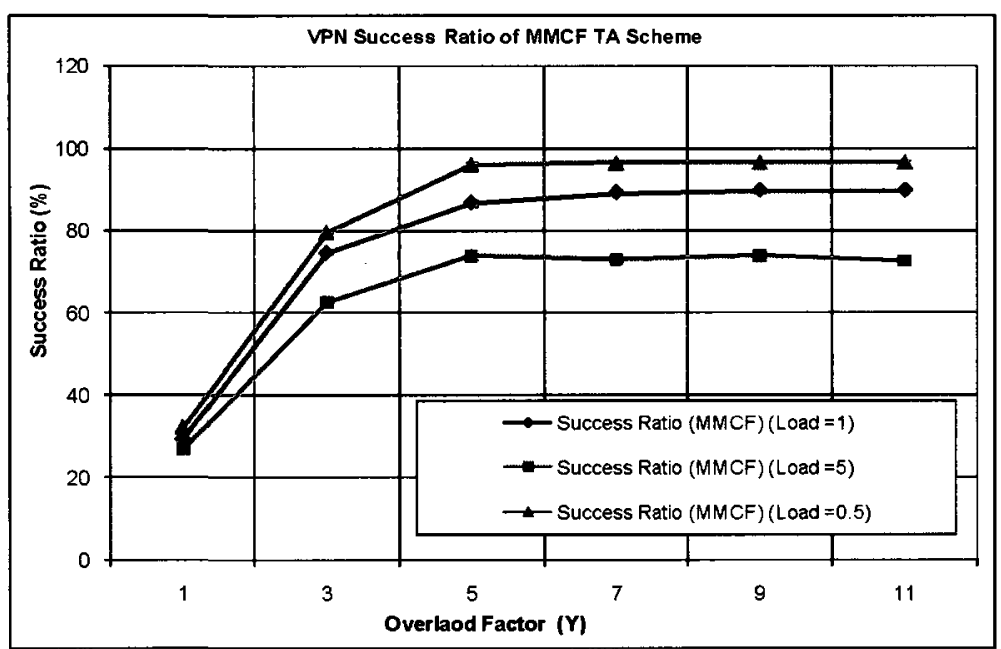

Figure 6-35: Objective 4(b), VPN call performance comparing success ratio of MMCF based abstraction scheme with varying oversubscription factor $(Y)$ with 95\% C.I (Network-1) 


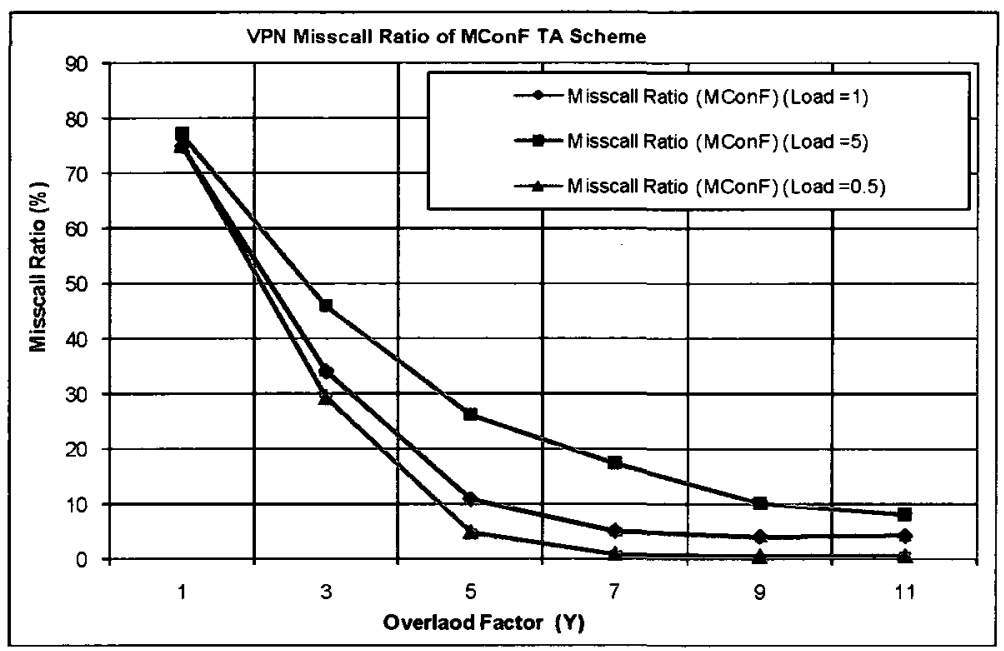

Figure 6-36: Objective 4(b), VPN call performance comparing misscall ratio of MConF based abstraction scheme with varying oversubscription factor $(Y)$ with 95\% C.I (Network-1)

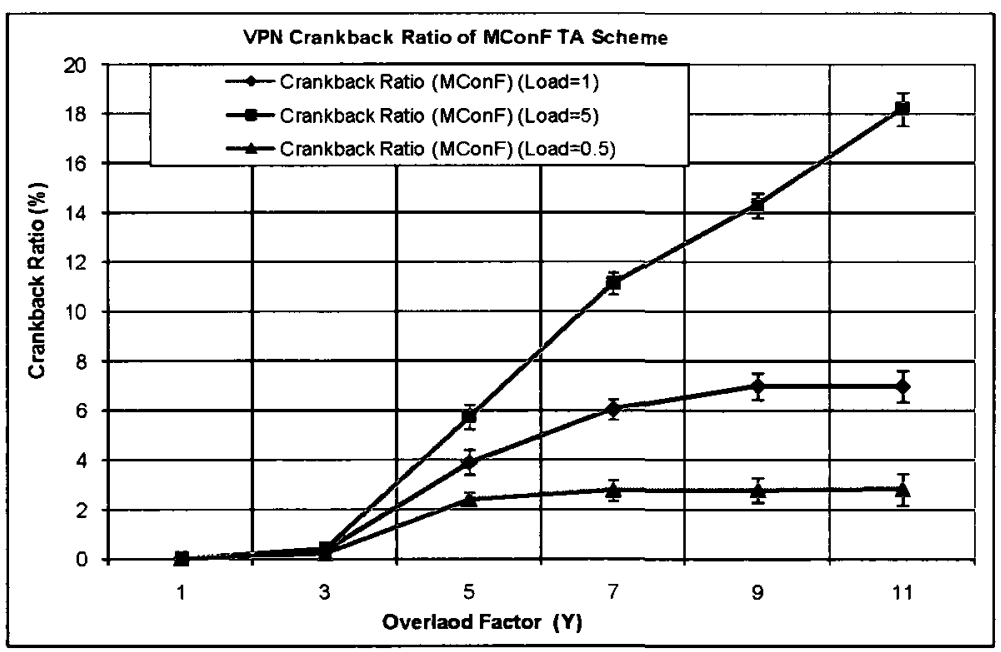

Figure 6-37: Objective 4(b), VPN call performance comparing crankback ratio of MConF based abstraction scheme with varying oversubscription factor (Y) with 95\% C.I (Network-1) 


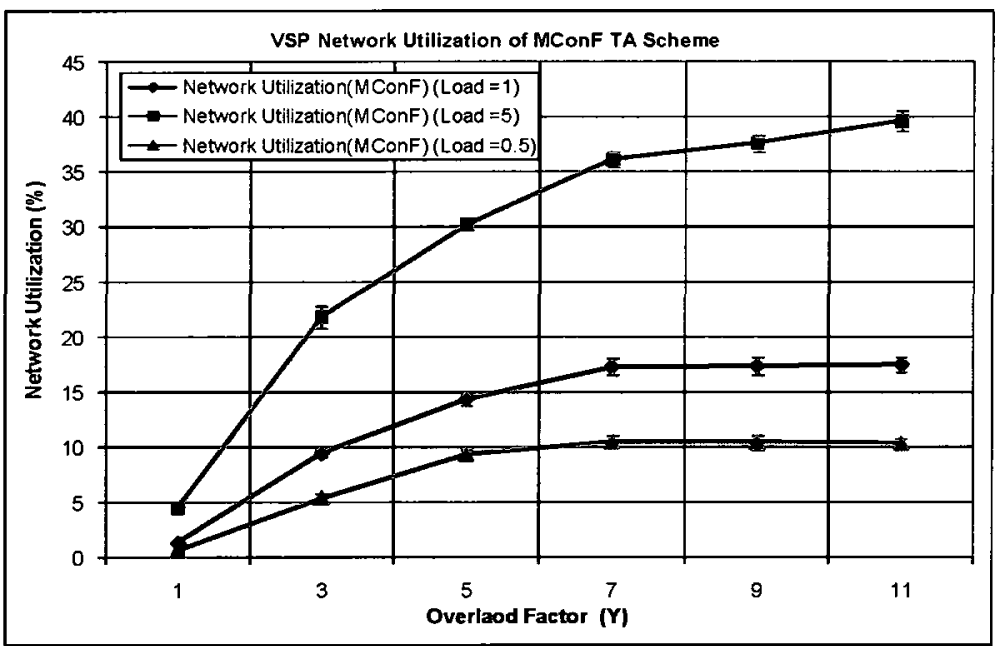

Figure 6-38: Objective 4(b), VSP network utilization comparison of MConF based abstraction scheme with varying oversubscription factor $(Y)$ with $95 \%$ C.I (Network-1)

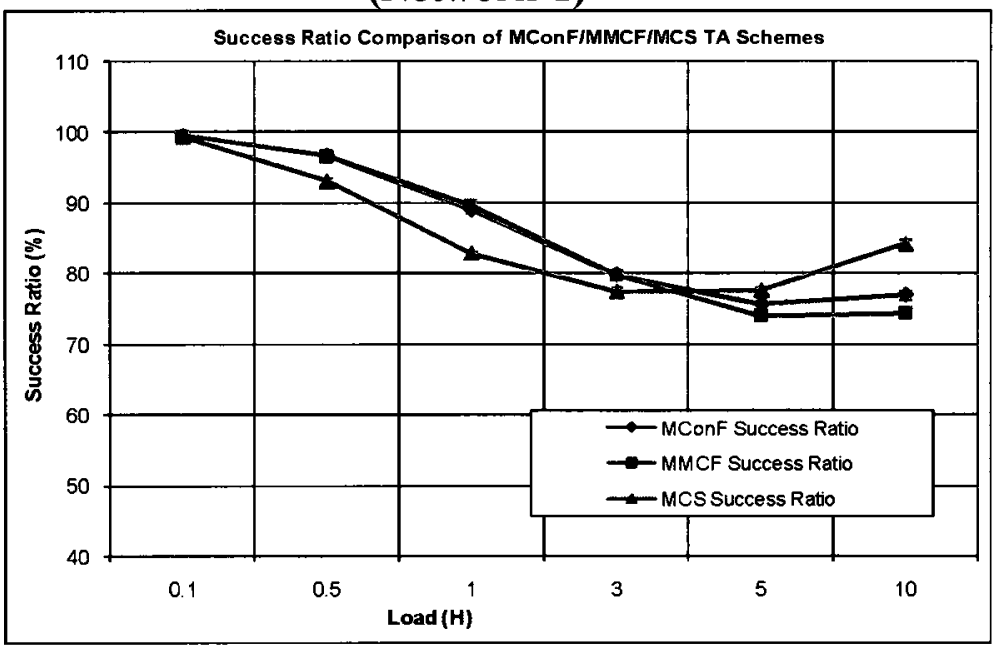

Figure 6-39: Objective 4(b), Success ratio comparison of MConF/MMCF/MCS TA schemes with varying mean holding time (H) with $95 \%$ C.I (Network-1) 


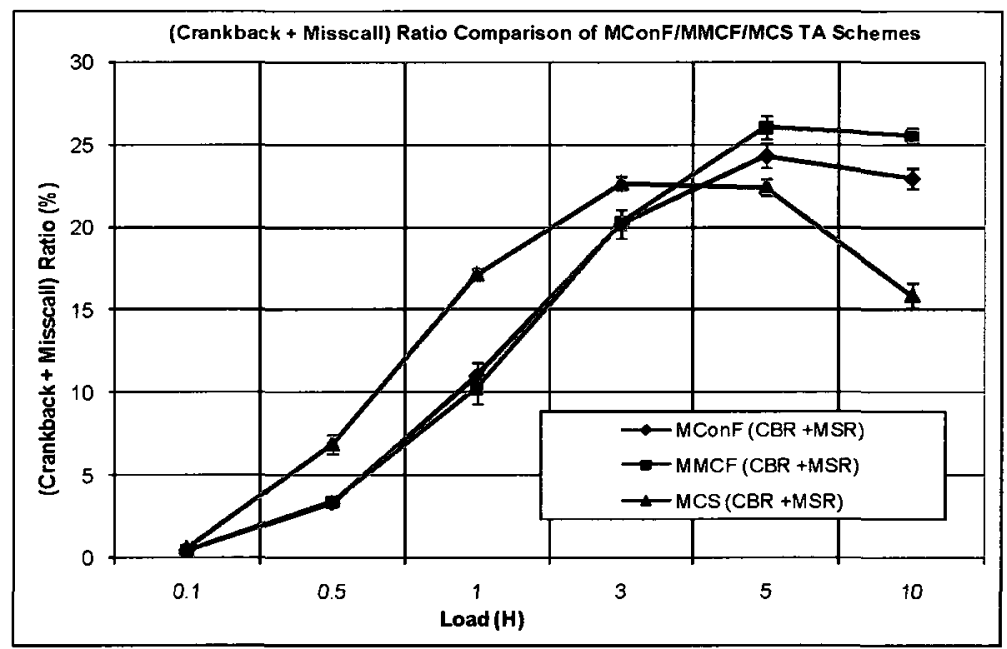

Figure 6-40: Objective 4(b), Success ratio comparison of MConF/MMCF/MCS TA schemes with varying mean holding time $(\mathrm{H})$ with $95 \%$ C.I (Network-1)

\section{Comparing the effect of link oversubscription for Network $2 \& 3$ for MConF and}

\section{MMCF scheme and its comparison to maximum capacity scheme}

Fig. A-9(a-b) compares the success and crankback ratio of MConF based scheme with varying link oversubscription factor $(Y)$ for Network-2. Fig. A-10(a-b) compares the misscall ratio and network utilization. The trends in these graphs with respect to improvement of MConF scheme's performance for the three cases of load conditions shown in the graphs is similar to that of Network-1. From the graphs, we note a significant improvement in terms of success ratio even at lower oversubscription factor, as in $Y=3$, because of significant improvement with respect to misscall ratio. With link oversubscription the gain in terms of success ratio achieved for both the MConF and MMCF scheme for the three load conditions is observed to be the same, overshadowing the difference in performance caused due to the performance of abstraction efficiency. The reason for saturation of the graphs after a certain value of oversubcription factor $(Y)$ 
is due to the saturation of crankback ratio. The decrease in the gain of success ratio with increasing network load is similar to the reasons discussed in the case of Network-1.

Fig. A-11(a-b) compares the success and (crankback + misscall) ratio of MConF and MMCF TA schemes with maximum capacity scheme for Network-3. In this case the oversubscription factor $(Y)$ is set to 9 for the centralized schemes. We note that at lower load conditions both the centralized and decentralized scheme perform equally well. However, the gap between the two widens at higher load conditions. This is because of the degradation of performance with respect to crankback ratio when oversubscription is enabled. The reason for this was discussed in the case of Network-1. The difference between the centralized and the maximum capacity scheme is in the range of $5 \%-10 \%$ at higher load conditions. However, this difference in performance of success ratio difference is significantly less compared to case when no link oversubscription is applied for the centralized schemes.

Fig. A-12(a-b) compares the performance of MConF based scheme with respect to success and crankback ratio for Network-3. Fig. A-13(a-b) compares the performance with respect to misscall ratio and network utilization. The performance trends in this case is noted to be similar to Network-1 and Network-2.

Fig. A-14(a-b) compares the success and (crankback + misscall) ratio performance of MConF, MMCF and maximum capacity scheme when link oversubscription is applied in the case of the centralized schemes. The oversubscription factor $(Y)$ for this case was also chosen to be 9 . As in the case of Network-2, the success ratio of the centralized schemes was observed to perform as good as (and in some cases 
better than) the maximum capacity scheme at low load conditions. However, the maximum capacity scheme can be observed to perform better than the centralized scheme at higher load conditions. This is because of the increase in crankback ratio with increasing load as discussed in the case of Network-1. In this case, at higher loads, the performance of the centralized schemes performs poorly by $10 \%$ compared to the maximum capacity scheme. However, this is better than $25 \%-30 \%$ degradation noted when no link oversubscription is applied.

\subsection{Summary}

In this chapter, we investigated the important problem of resource partitioning and TA generation in a centralized manner in the context of the TA service to the VPNs using the multicommodity flow theory. In this context, we defined a problem called the VPN core capacity sharing (VPN-CS) problem. Considering the objectives of the VPN-CS problem, we first proposed a method based on the maximum concurrent flow theory, and later improved it by proposing the use of the maximum multicommodity flow theory. In order to address the fairness issue associated with the MMCF based approach, a new problem called the fair partitioning problem was formulated. We studied this problem by proposing the bounded MMCF formulation, wherein two variations were proposed. In addition, we also proposed a heuristic called the flow balancing scheme to address the fair partitioning problem for online implementation.

Performance analysis was conducted where we studied the proposed schemes using offline implementation with an LP tool and discrete event driven simulation. As 
expected, MConF achieved the best fairness, while MMCF achieved the maximum abstraction efficiency.

From the simulation analysis, we observed that the MMCF based partitioning approach resulted in better call performance than the $\mathrm{MConF}$ based partitioning scheme. The MMCF based partitioning algorithm performed about $5 \%$ better than the MConF approach with respect to both VPN call performance and network utilization metrics. With respect to abstraction efficiency, we observed that MMCF's performance was $37 \%$ better than MConF; however, in terms of fairness, the standard deviation of the commodity flows generated by MConF was on an average $75 \%$ less than the standard deviation of the flows generated by MMCF. With flow balancing enabled, the simulation analysis also showed that the fairness issue of MMCF could be improved by about $25 \%$ with no significant impact on the overall call performance of the VPNs or the core network utilization.

Comparing the performance of the two fairness improvements suggested so as to improve MMCF's flow fairness for offline implementation, the second variation, namely, the MB-2 method that used the MConF and MMCF flows to define the lower and upper bounds of the commodity flows, struck the best balance between the abstraction efficiency, matching that of MMCF formulation, and closest performance in terms of faimess compared to MConF based formulation.Comparing the performance of the centralized and decentralized abstraction schemes, we observed that the performance of the maximum capacity scheme exhibited better performance, both in terms of call performance and network utilization, than the MConF and MMCF based TA schemes. 
We also studied how this can be addressed by oversubscribing the residual capacity of core network links before applying the centralized abstraction schemes. We observed that the call performance and core network utilization showed significant improvement over a range of the oversubscription factors, demonstrating this as a practical way to overcome the conservative nature of the centralized mode of VPN TA generation.

In summary, the study in this chapter demonstrated how abstractions can be generated in a centralized manner to enable dynamic bandwidth service using the multicommodity flow theory and applying fully polynomial and tuneable FPTAS for online implementation. 


\section{Chapter 7}

\section{Conclusions and Future Work}

\subsection{Conclusions}

In this research we showed how the notion of topology abstraction can be applied to share VSP's sensitive core topology information with the VPNs in a practical and scalable manner, and how it can be realized as a service offering applying suitable SLA metrics in the context of a current managed IP-VPN solution. We realized this novel TA service definition in the context of a challenging framework called the managed dynamic VPN framework (MDVF) based on an existing provider provisioned IP-VPN solution. To address the issue of fairness and service differentiation we proposed to generate the abstractions using a three-phase TA generation process.

In Chapter 4 we demonstrated, through simulation analysis, the feasibility and usefulness of the TA service, and its ability to generate service differentiation incorporating the notions of fairness among the VPNs subscribing to this service. Here, we also noted the drawbacks associated with the TA service in the form of increased misscall ratio and reduced network utilization compared to the case when no TA service 
is enabled. However, the performance deterioration in terms of these metrics is smaller compared to the significant gain achieved in terms of overall success ratio performance when TA service is provided. This achieves the objective of improving the routing efficiency of VPNs considerably.

In Chapter 5 we studied the problem of TA generation using decentralized schemes. Here, we proposed three heuristic solutions. These abstraction schemes exhibited different performance tradeoffs because of their nature of either being conservative or aggressive in terms of exposing resources to the VPNs. Here we observed that the Steiner tree scheme struck a good balance in terms of success, misscall and crankback ratio compared to maximum capacity and mixed bound schemes.

We next studied in Chapter 6 the problem of TA generation using the centralized approach wherein the partition graphs for the abstract topology generation are computed in a centralized manner. Here we first proposed a solution based on the maximum concurrent flow (MConF) theory. It was later improved by proposing the use of the maximum multicommodity flow (MMCF) theory. In order to address the fairness issue associated with the MMCF based approach, we proposed the bounded MMCF formulation wherein two variations of the MMCF formulation were proposed. In addition, the flow balancing heuristic was also proposed for online implementation. Both these proposals were noted to improve the commodity flow fairness of the MMCF solution quite significantly.

Finally, we compared the performance of centralized and decentralized based abstraction schemes where we observed that the performance of the decentralized scheme 
was better both in terms of call performance and network utilization than centralized abstraction schemes. We showed how this difference in performance can be addressed, and demonstrated that the centralized scheme could perform as well as or better than the decentralized schemes for various load conditions by the careful choice of the link oversubscription factor.

\subsection{Future Work}

In the following, we discuss some possible extensions of the ideas presented in this thesis for future investigation.

\subsubsection{Topology Abstraction Service with Multiple QoS}

\section{Metric Aggregation}

In this thesis, we limited our study to the abstraction of link capacity information to the VPNs in a fair manner. VPN multimedia applications, in addition to being sensitive to bandwidth are also sensitive to delay, jitter and packet loss. This is particularly true for multimodal applications with voice and video components. End-to-end delay is an aggregation of both propagation delay due to physical distance as well as queuing delay introduced by routers in the core network, making it a time varying metric. Similarly, jitter and packet loss ratios are also a function of the network level congestion and the state of queues in intermediate routers. Extending the TA-SLA parameters to include these QoS parameters, i.e. delay, jitter, and packet loss, would be very valuable to the 
VPNs deploying multimedia applications that typically have constraints with respect to more than one QoS metrics for multimedia applications.

To associate multiple QoS metric with the abstract topology, the problem here is to study algorithms that can summarize delay, jitter, and packet loss as abstract metrics and associate them with the virtual links of the TAs generated for the VPNs. The problem of single and two abstraction metrics has been well studied in the context of PNNI. The related literature was discussed in Section 2.7. The goal here, in the context of the TA service to the IP-VPNs, will be to extend the proposals made in the context of hierarchical routing or propose new schemes, which could summarize multiple QoS metrics and associate them with virtual links of the abstractions provided to the VPNs; this should be done considering the performance and fairness aspect of VPN TA service. The objectives of these algorithms should be to minimize algorithmic complexity and control overhead, while minimizing the information loss due to QoS metric aggregation.

\subsubsection{Routing Using Partition Subgraphs Generated by Centralized Topology Abstraction Scheme}

In the centralized mode of generating TA, one of the intermediate but a most important step is the generation of VPN partition subgraphs. We used these non-overlapping partition subgraphs to generate TAs for each VPN. A further extension of the use of these subgraphs is to determine paths to route VPN calls during the event of a bandwidth request from a VPN. 
Routing using partition subgraphs can be realized in the context of the MDVF either in a decentralized or centralized manner. In a decentralized manner, the border PE nodes are provided with the partition subgraph information which can be used to determine the paths during a bandwidth request by the VPNs; and in a centralized approach, the path request from the border node is forwarded to the CS which computes the path using the partition subgraph computed for that VPN, however, this approach has to account for increased overhead and path signaling delay caused by the interaction between the CS and the border node for each VPN call request.

Using the partition subgraph for routing for VPN requests is expected to reduce resource contention as they are generated in a non-overlapping manner during the graph partition computation phase by the CS, and hence, could improve VPN call performance compared to the case when the routing is conducted by default using the core topology for all the VPNs. However, this approach could also prove to be conservative because of poor statistical multiplexing of the resources, due to routing decisions derived from graph partitions. This drawback could be overcome by applying intelligent resource management strategies either at the border node or by the CS over the VPN subgraph partitions incorporating notions of resource sharing, prioritizing, service differentiation, and preempting resources based on the priority of a VPN call request. These resource management strategies can be further enhanced by considering statistic such as load offered and current usage of resource by the VPNs. 


\subsubsection{Extending Topology Abstraction Service to Layer 1 VPNs}

This thesis studied TA service in the context of IP-VPNs. An extension of this study is application of this idea in the context of L1 VPNs [RFC4847]. Constructing a virtual topology in order to satisfy the static traffic matrix specification of customers over optical networks based on TDM or WDM based transport is a well-researched topic. [Dut00] provides a good survey of solutions proposed in this area of research.

In a dynamic call scenario, where the future traffic requirement cannot be estimated and resources are committed and torn down on demand, there is a need for a strategy that would enable L1 VPN customers to seek resources with the knowledge of the state of the transport network, which is similar to the problem addressed in this thesis in the context of IP-VPNs. Hence, the TA service proposed in this research can also be applied in the context of L1 VPNs as a means of disseminating topology and network state information as abstract topologies to the bordering $\mathrm{CE}$ nodes, which could use it to compute the feasibility of meeting its QoS demands in the core network.

In the case of L1 VPNs, the problem of generating topology abstractions is a more difficult problem, since it needs to be solved considering constraints of transport networks, such as wavelength or time-slot continuity constraints, such that the virtual links in the abstract topology guarantees with good probability the existence of a path in the physical network between the end points of the virtual link. Further, as in the IP-VPN case, the construction of the abstract topology can also be influenced by the negotiated 
TA-SLA parameters and VSP's fairness policies applied while constructing the abstract representations of the underlying L1 transport network for the VPNs.

Realization of TA service in the context of L1 VPN is possible by suitable extensions to the GMPLS [RFC3945] based routing and signaling framework proposed to control various circuit switched transport technologies like optical transport networks. As in the case of IP-VPNs, even in this case, abstract topology information from PE nodes to VPN CE nodes can be flooded by appropriate extensions to the OSPF-TE [RFC4203] protocol. [Rav04] provides an investigation of this problem of enabling TA service to the edge IP routers supported over a L1 optical network managed by a GMPLS based control plane framework. 


\section{Appendix A:}

\section{A.1 Simulation Results}
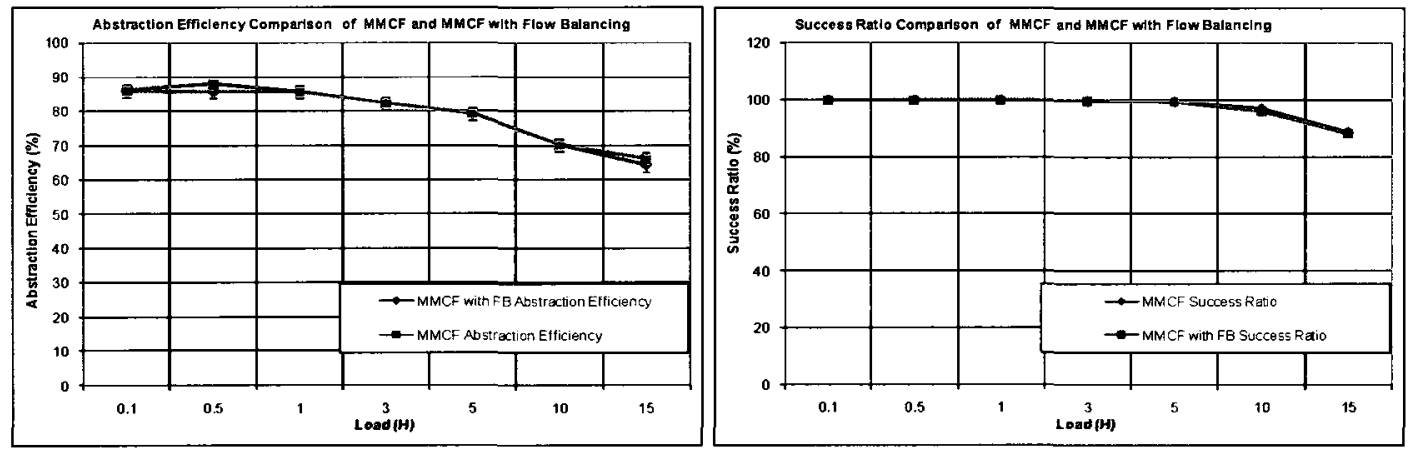

Figure A-1(a-b): Objective 3(b), Comparing VSP abstraction efficiency and success ratio of $\mathrm{MMCF}$ with and without Fair Balancing with varying mean holding time (H) with 95\% C.I (Network-2)

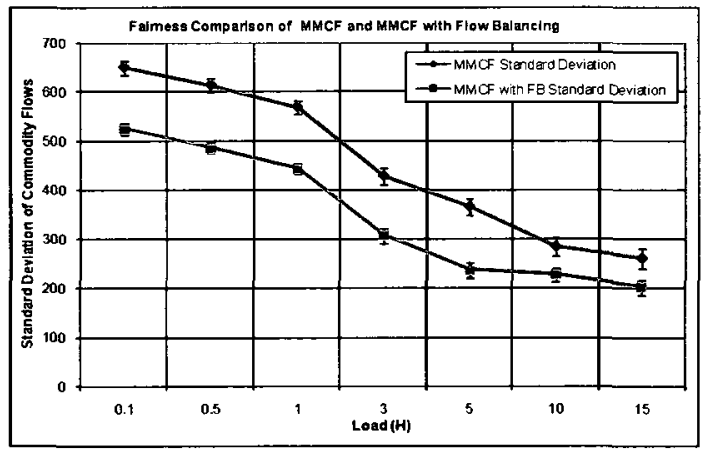

Figure A-2: Objective 3(b), Comparing commodity fairness of MMCF with and without Fair Balancing with varying mean holding time (H) with $95 \%$ C.I

(Network-2) 

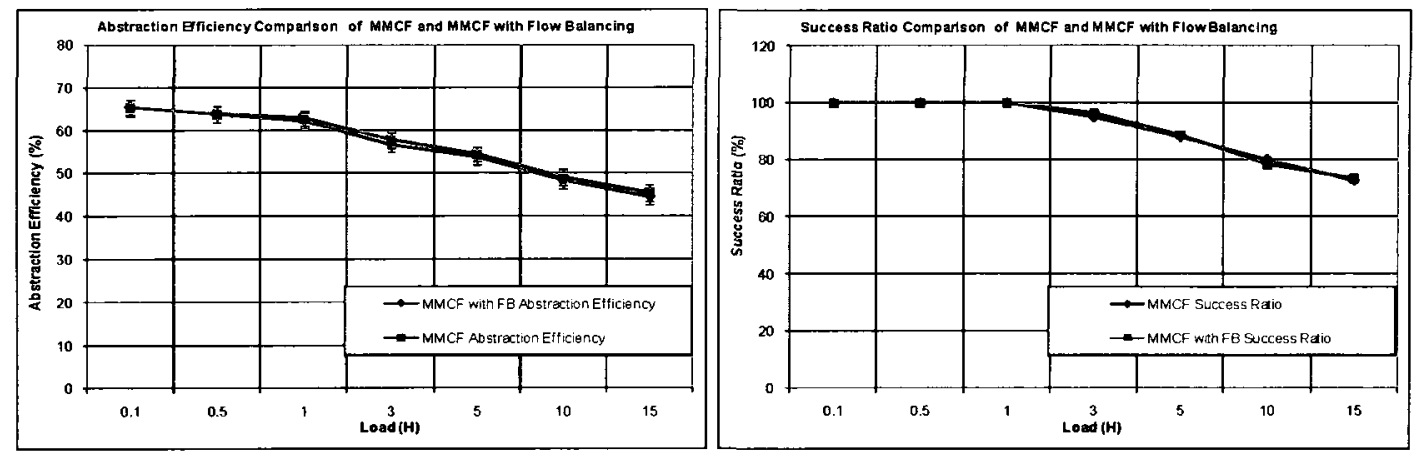

Figure A-3(a-b): Objective 3(b), Comparing VSP abstraction efficiency and success ratio of MMCF with and without Fair Balancing with varying mean holding time (H) with 95\% C.I (Network-3)

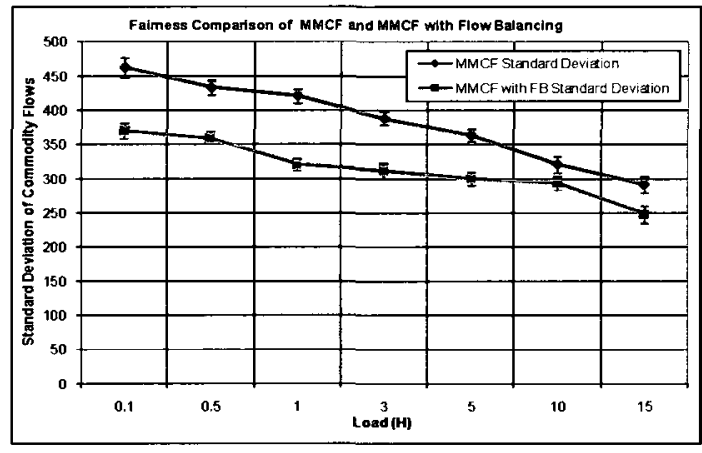

Figure A-4 : Objective 3(b), Comparing commodity fairness of MMCF with and without Fair Balancing with varying mean holding time (H) with $95 \%$ C.I (Network-3)
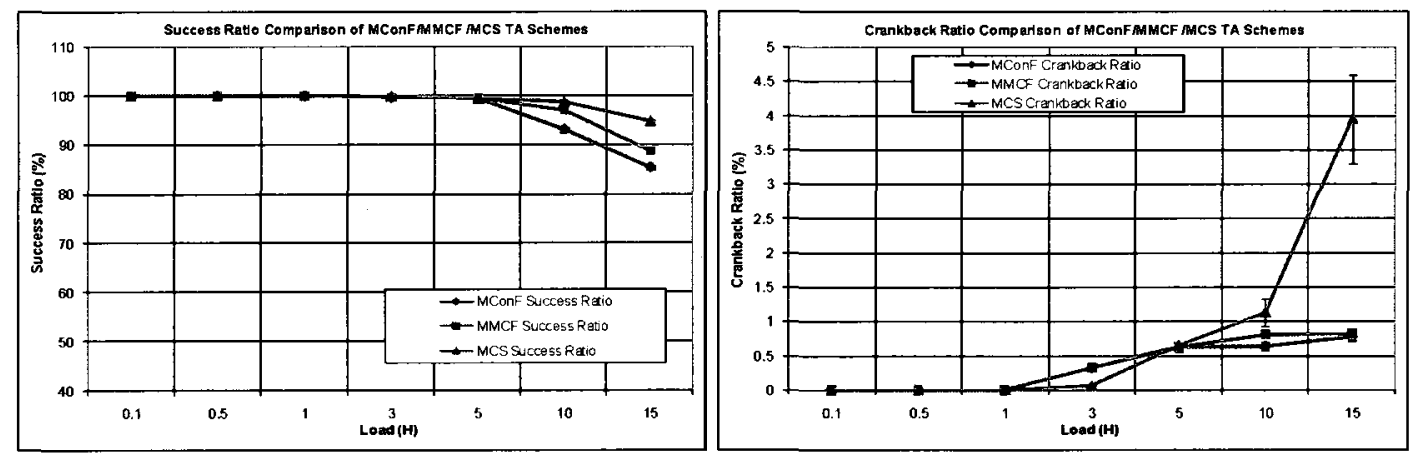

Figure A-5(a-b): Objective 4(a), VPN call performance comparing success ratio and crankback ratio of maximum capacity, MConF and MMCF based TA schemes with varying mean holding time (H) with $95 \%$ C.I (Network-2) 

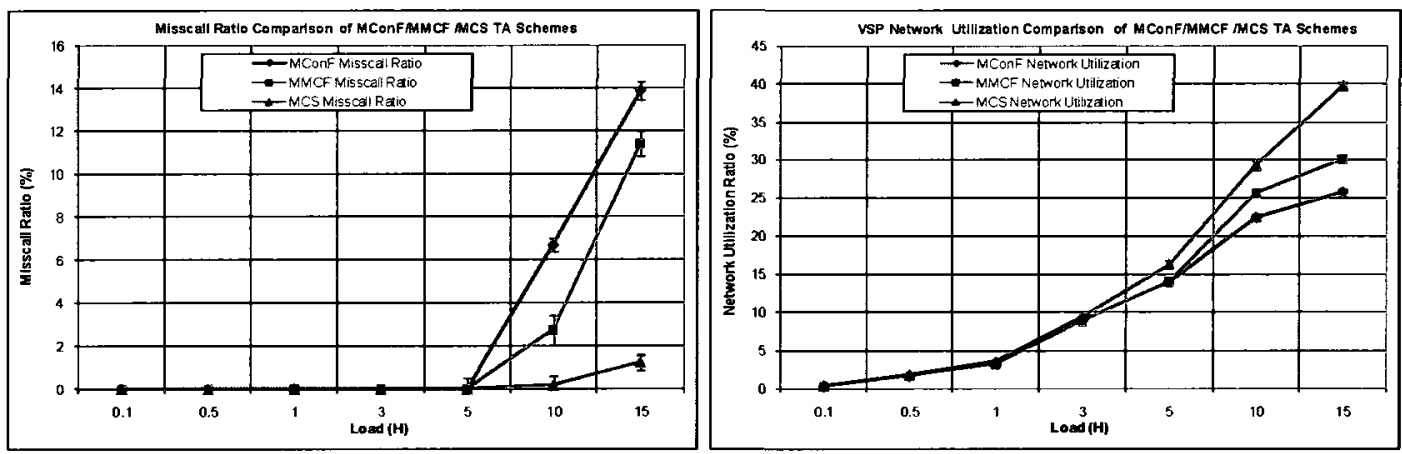

Figure A-6(a-b): Objective 4(a), VPN call performance comparing misscall ratio and VSP network utilization of maximum capacity, MConF and MMCF based TA schemes with varying mean holding time (H) with $95 \%$ C.I (Network-2)
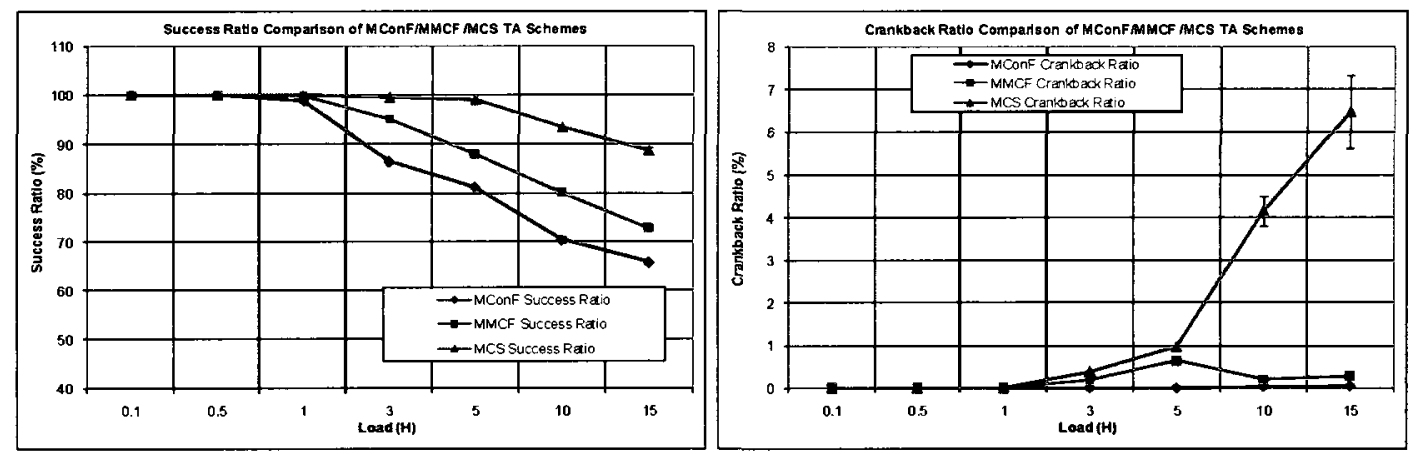

Figure A-7(a-b): Objective 4(a), VPN call performance comparing success ratio and crankback ratio of maximum capacity, MConF and MMCF based TA schemes with varying mean holding time $(\mathrm{H})$ with $95 \%$ C.I (Network-3)
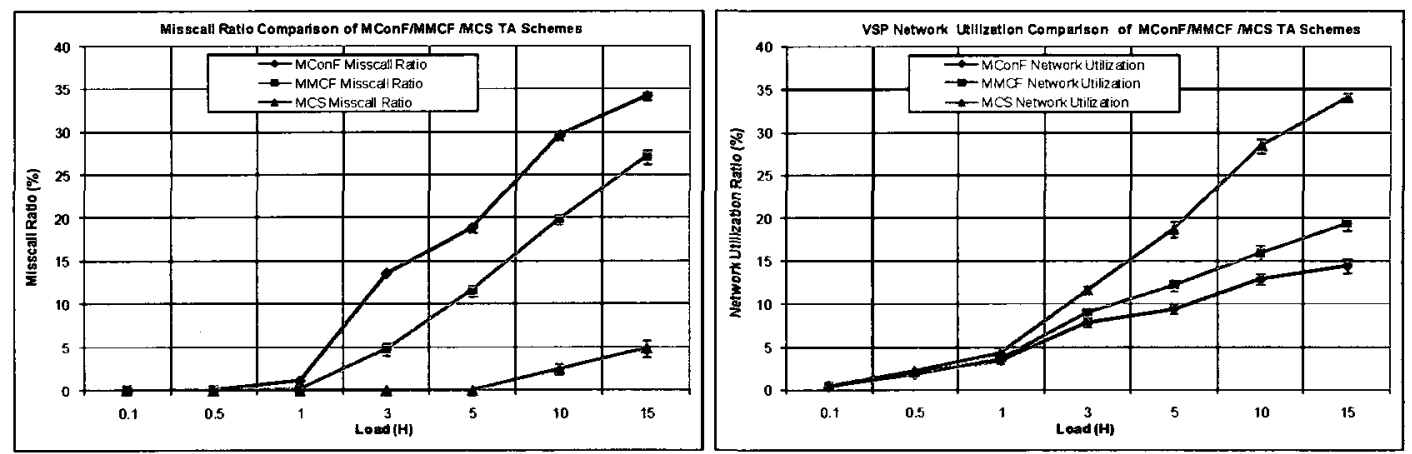

Figure A-8(a-b): Objective 4(a), VPN call performance comparing misscall ratio and VSP network utilization of maximum capacity, MConF and MMCF based TA schemes with varying mean holding time (H) with $95 \%$ C.I (Network-3) 

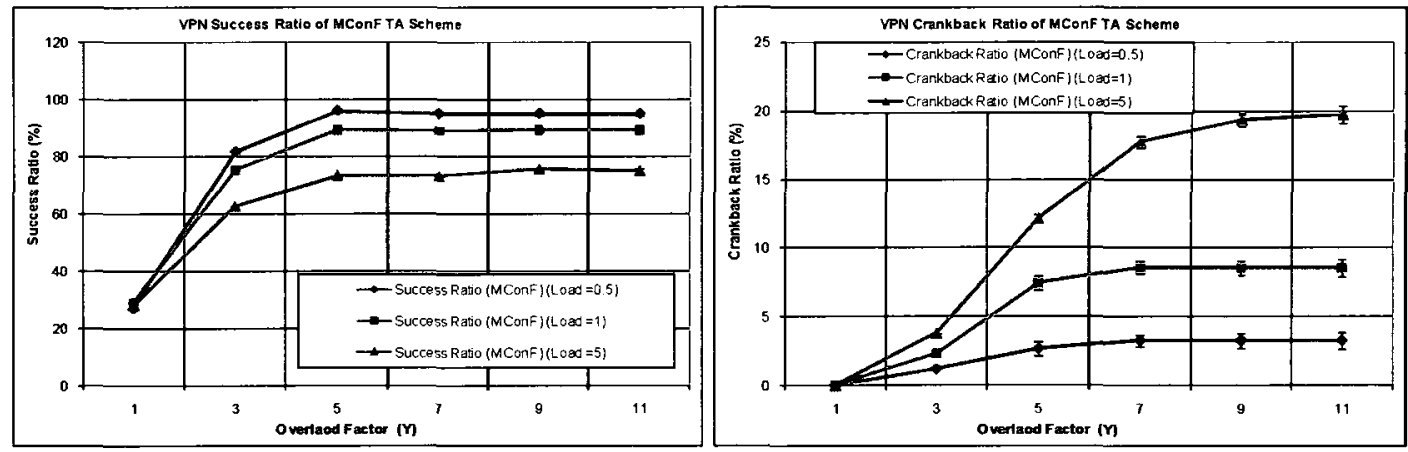

Figure A-9(a-b): Objective 4(b), VPN call performance comparing success ratio and crankback ratio of MConF based abstraction scheme with varying oversubscription factor (Y) with 95\% C.I (Network-2)
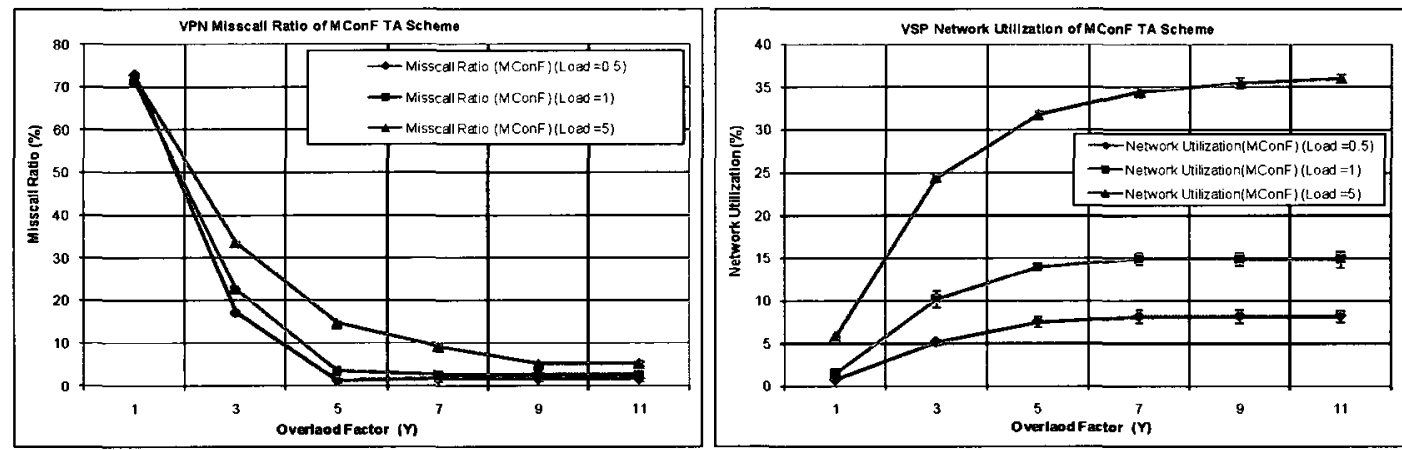

Figure A-10(a-b): Objective 4(b), VPN call performance comparing misscall ratio and VSP network utilization of MConF based abstraction scheme with varying oversubscription factor (Y) with $95 \%$ C.I (Network-2)
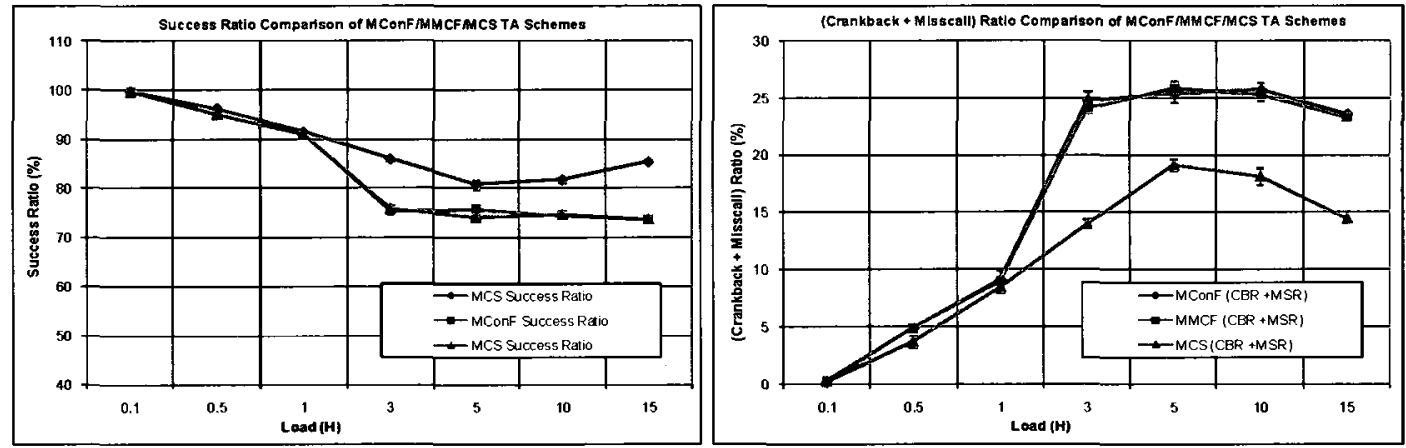

Figure A-11(a-b): Objective 4(b), Success ratio and crankback ratio comparison of MConF/MMCF/MCS TA schemes with varying mean holding time $(\mathrm{H})$ with $\mathbf{9 5 \%}$ C.I (Network-2) 

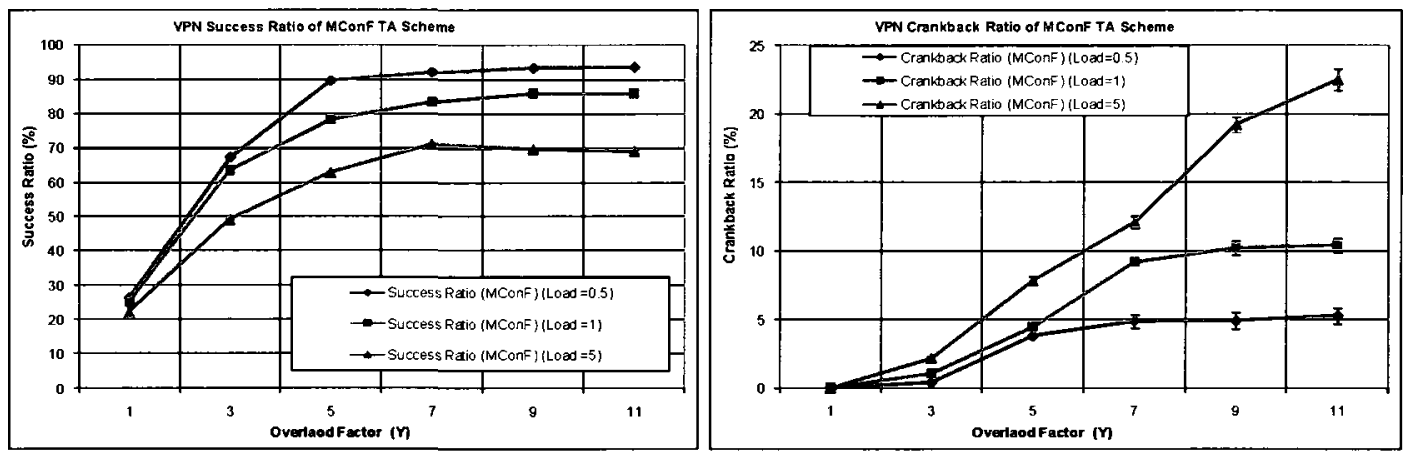

Figure A-12(a-b): Objective 4(b), VPN call performance comparing success ratio and crankback ratio of $M C$ ConF based abstraction scheme with varying oversubscription factor (Y) with $95 \%$ C.I (Network-3)
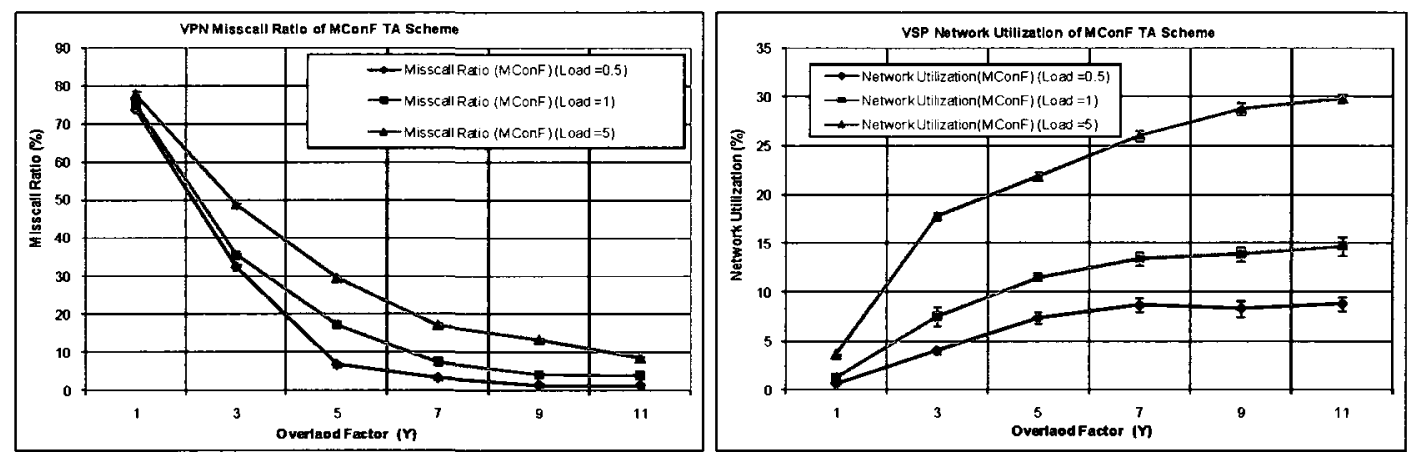

Figure A-13(a-b): Objective 4(b), VPN call performance comparing misscall ratio and VSP network utilization of MConF based abstraction scheme with varying oversubscription factor (Y) with $95 \%$ C.I (Network-3)
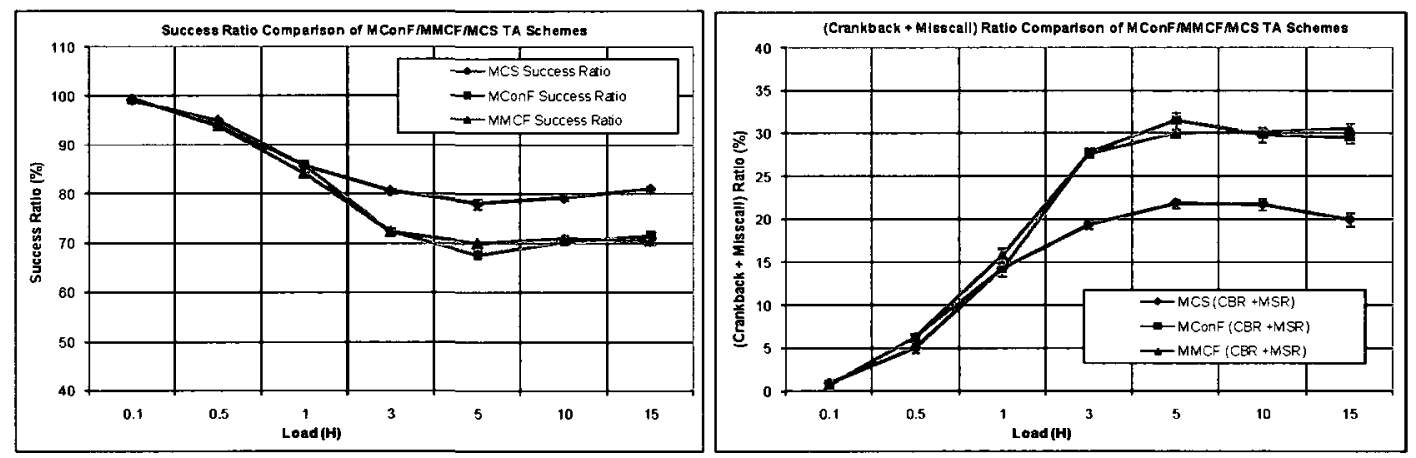

Figure A-14(a-b): Objective 4(b), Success ratio and crankback ratio comparison of MConF/MMCF/MCS TA schemes with varying mean holding time $(\mathrm{H})$ with $95 \%$ C.I (Network-3) 


\section{A.2 Widest Path Algorithm}

For a graph $G(V, E)$, this algorithm [Ahu93] computes a widest path tree from a source node $s$ to all nodes spanning $V$. The algorithm applies a greedy approach in order to build the spanning tree. In each iteration of the algorithm, a node $x$ with the maximum capacity label $w(x)$ is chosen from the temporarily labeled set $S^{\prime}$ and labeled permanently, while modifying the labels of its neighbors if necessary. The algorithm ends when all the nodes are permanently labeled. The complexity of the algorithm using a Fibonacci heap data structure is $O\left(|E|+|V|^{*} \log (|V|)\right)$.

\section{Widest Path Tree Algorithm}

Input: $G(V, E)$, source $s$.

Output : Widest path tree rooted at $s$ spanning all nodes in $V$.

begin

$\mathrm{S}:=\phi ; \mathrm{S}^{\prime}:=V ;$

$w(v):=0$ for each node $v \in V$;

$w(s):=\infty$ and $\operatorname{pred}(s):=0 ;$

while $|\mathrm{S}|<|V|$ do

begin

let $x \in \mathrm{S}^{\prime}$ be a node for which $w(x)=\max \left\{w(j): j \in \mathrm{S}^{\prime}\right\}$;

$\mathrm{S}:=\mathrm{S} \cup\{x\}$

$\mathrm{S}^{\prime}:=\mathrm{S}^{\prime}-\{x\}$

for each $(x, j) \in E(x)$ do

if $w(j)<\min \left(w(x), r_{x, j}\right)$ then $w(j)=\min \left(w(x), r_{x_{j} j}\right)$

and $\operatorname{pred}(j):=x$; 
end;

end;

\section{A.3 Shortest Augmenting Path Algorithm}

This algorithm [Ahu93] computes the maximum flow possible from source node $s$ to destination node $t$. The maximum flow is computed using the principle of flow augmentation from source node $s$ to target node $t$ in the residual graph. The algorithm works by applying either advance or retreat operation along the shortest path between $s$ and $t$ in a residual graph so as to augment the flow. The algorithm continues until there are no more admissible paths from $s$ to $t$. The algorithm has a complexity of $O\left(|V|^{2 *}|E|\right)$.

\section{Shortest Path Augmenting Maximum Flow Algorithm}

Input: $\mathrm{G}(\mathrm{V}, \mathrm{E})$, source $s$, destination $t$.

Output : Maximum flow from $s$ to $t$.

(Shortest Augmenting path Algorithm, Page 215, [Ahu93])

\section{A.4 Maximum $M$-Route Flow Algorithm}

This algorithm [Agg01] computes the maximum $M$-Route flow from source node $s$ to destination node $t$. In each iteration $i$, the maximum flow $v_{i}$ is computed over a truncated graph $\left(G, p_{i}\right)$, where links in graph $G$ are upper bounded by a value $p_{i}$. The algorithm terminates, when it satisfies the condition $v_{i}=M^{*} p_{i}$. This algorithm finds the $M$-Route 
flow in at most $M$ max-flow computation between $s$ and $t$, hence, its complexity when the above discussed shortest augmenting path algorithm is applied is $O\left(M^{*}\left|\eta \eta^{2 *}\right| E \mid\right)$.

\section{M-Route Flow Algorithm}

Input: $G(V, E)$, source $s$, destination $t, M$.

Output : Maximum $M$-Route flow from $s$ to $t$.

begin

$v_{0}:=$ Compute maximum flow between nodes $s, t$ in graph $\mathrm{G} ;$

set $i:=1$;

let $p_{i}:=v_{0} / M$;

truncate_graph $\left(G, p_{i}\right)$;

$v_{i}=\max$-flow $(G, s, t)$;

while $v_{i} \neq M^{*} p_{i}$ do

begin

$i=i+1 ;$

$p_{i}=v_{i} / M$

truncateGraph $\left(G, p_{i}\right)$;

$v_{i}=\max$-flow $(G, s, t)$;

if $v_{i}=M^{*} p_{i}$, then $v_{i}$ is the maximum value of the $M$-Route flow. Stop.

if $v_{i}<M^{*} p_{i}$, then $p_{i}=\frac{\left(v_{i}-i^{*} p_{i}\right)}{(M-i)}$

end;

end;

truncate_graph $(G, p)$

begin

for each $e \in E$ do 


$$
\text { if } r_{i, j}>p, r_{i, j}=p
$$

end ;

\section{A.5 Nearest Node First Steiner Tree Algorithm}

This heuristic [Kod00] computes a tree rooted at $s$, spanning all nodes in $S^{\prime}$ while minimizing the cost of building the tree. In each iteration, the algorithm follows a greedy approach by computing the shortest path from source node $s$ until the first node $r$, which is not yet in the tree, is reached. In order to encourage the future iteration to reuse these links, the edge cost of the links of the paths to $r$ is set to 0 . The algorithm ends when all the nodes in set $S^{\prime}$ are part of the tree. The complexity of the algorithm is $O\left(^{*}\left|S^{\prime}\right|\left(|E|+\left|\eta^{*} \log \right| V \mid\right)\right)$.

\section{Nearest Node First Steiner Tree Algorithm}

Input: $G(V, E)$, source $s$, node set $S^{\prime}$.

Output: A low cost Steiner tree rooted at $s$ spanning all nodes in $S^{\prime}$.

begin

$X:=S^{\prime}$

while $X \neq \phi$

Run Dijkstra's shortest path algorithm with source $s$ till a node ; $r \in S^{\prime}$ is reached;

Add the path from source node $s$ to $r$ to the Steiner tree built so far ;

Set the cost of all the edges along this path to zero;

$X:=X-\{r\}$; 
end while;

end;

\section{A.6 Maximum Multicommodity Flow FPTAS}

The algorithm [Fle99] presented below is a FPTAS for the MMCF problem. For the algorithm $\varepsilon$ is chosen based on desired approximation as in $(1-\varepsilon)^{*} O P T$ (where $O P T$ is the optimal aggregate flow of the MMCF problem). Inside the outer most loop, the algorithm cycles through the set of commodities, and for each commodity $k$, we stick with the commodity until the shortest source to sink path for that commodity is above a $(1+\varepsilon)$ factor times a lower bound estimate of the overall shortest path. Then we begin the iteration for the next commodity. The algorithm has a complexity of $O\left(\varepsilon^{-2 *}\left(|E|^{2}+|K|^{*}|E|\right)\right)$.

\section{Maximum Multicommodity Flow FPTAS}

Input: $G(V, E)$, capacities $r(e)$, commodity pairs $\left(s_{k}, d_{k}\right) \quad 1 \leq k \leq K$, accuracy $\varepsilon$.

Output: Primal $x(e) \forall e \in E$ and Dual solutions $l(e) \forall e \in E$.

begin

Initialize $l(e):=\delta$, where $\delta=(1+\varepsilon) /\left((1+\varepsilon)^{*}|V|\right)^{1 / \varepsilon}, x \equiv 0$;

for $i=1$ to $\log _{(1+\varepsilon)}\left(\frac{(1+\varepsilon)}{\delta}\right)$

$$
\begin{aligned}
& \text { for } j=1 \text { to } k \\
& \qquad \begin{aligned}
p:= & \text { shortest path in } P_{j} \text { using } l ; \\
& \text { while } l(p)<\min \left\{1, \delta(1+\varepsilon)^{i}\right\}
\end{aligned}
\end{aligned}
$$


$u:=\min _{e \in p} r(e)$

for each $e \in p$

$$
\begin{aligned}
& x(e):=x(e)+u ; \\
& l(e)=l(e)\left(1+\frac{\varepsilon^{*} u}{u(e)}\right) ;
\end{aligned}
$$

$p:=$ shortest path in $P_{j}$ using $l$;

end while;

end for ;

//obtain primal feasible solution

for each $e \in E$

$$
x(e)=\frac{x(e)}{\log _{(i+\varepsilon)} \frac{(1+\varepsilon)}{\delta}}
$$

end for ;

end;

\section{A.7 Maximum Concurrent Flow FPTAS}

The algorithm [Fle99] presented below is a FPTAS for MConF problem. For the algorithm, $\varepsilon$ is chosen based on desired approximation as in $(1-\varepsilon)^{*} O P T$ (where $O P T$ is the optimal throughput of the MConF problem). In each phase, there are $k$ iterations. In each iteration $j$, the objective is to route $D(k)$ units of flow from commodity $j$ between $s_{j}$ to $t_{j}$. For this, a shortest path $P$ from $s_{j}$ to $t_{j}$ is computed in each step using the current length function. Let $u$ be the capacity of this path. Then, the minimum of $u$ and the remaining demand is sent along this path. The dual variables $l(e)$ is increased as shown in 
the pseudo code, and $z_{j}$ is set equal to the length of the new minimum length path from $s_{j}$ to $t_{j}$. The phase ends when the dual objective function value is at least 1 . This algorithm has a complexity of $O\left(\varepsilon^{-2 *}\left(|E|^{2}+|K|^{*}|E|\right)\right)$.

\section{Maximum Concurrent Flow FPTAS}

Input : Input : $G(V, E)$, link capacities $r(e)$, commodity pairs $\left(s_{k}, d_{k}\right) \quad 1 \leq k \leq K$ with demand $D(k)$, accuracy $\varepsilon, P_{k}$ (Set of paths for commodity $k$ ) .

Output: Primal $x(e) \forall e \in E$ and Dual solutions $l(e) \forall e \in E$.

begin

Initialize $l(e)=\delta / r(e) \forall e$, where $\delta=\left(\frac{|E|}{(1-\varepsilon)}\right)^{-1 / \varepsilon}, x:=0$;

while $\sum_{e \in E} r(e)^{*} l(e)<1$

for $j=1$ to $k$ do

$d_{j}^{\prime}:=D(j)$;

while $\sum_{e \in E} r(e)^{*} l(e)<1$ and $d_{j}^{\prime}>0$

$p:=$ compute shortest path between $s_{j}$ to $t_{j}$ using $l$;

$u:=\min \left\{d_{j}^{\prime}, \min _{e \in p} r(e)\right\}$;

$d_{j}^{\prime}=d_{j}^{\prime}-u$

for each $e \in p$

$$
\begin{aligned}
& x(e):=x(e)+u ; \\
& l(e)=l(e)\left(1+\frac{\varepsilon^{*} u}{u(e)}\right) ;
\end{aligned}
$$

end while ;

end while ; 
//obtain primal feasible solution

for each $e \in E$

$$
x(e)=\frac{x(e)}{\log _{(i+\varepsilon)} \frac{1}{\delta}}
$$

end for ;

end; 


\section{References}

[Awe98] B. Awerbuch and Y. Shavitt, "Topology Aggregation for Directed Graph", IEEE/ACM Transactions on Networking, Vol. 9, Issue 1, pp. 82 - 90, Feb. 2001.

[Ahu93] Ravindra K. Ahuja, Thomas L. Magnanti, and James B. Orlin, "Network Fows: Theory, Algorithms, and Applications", Prentice-Hall, 1993.

[Au99] T. Andrew Au, “Topological Considerations of ATM Network", International Journal of Network Management, Vol. 9, pp. 347-358, 1999.

[Agg01] Charu C. Aggarwal and James B. Orlin, "On Multiroute Maximum Flows in Networks", IEEE Networks, Vol. 39(1), pp. 43-52, Nov. 2001.

[Ane99] Nikolaos Anerousis, "Dynamic Virtual Network Dimensioning in Cost Sensitive Environments", Proceedings of IEEE GLOBECOM, Vol. 2, pp. 1511 - 1516, 1999.

[Bra01] Torsten Braun, Manuel Guenter, and I. Khalil, "Management of Quality of Service Enabled VPN's", IEEE Communication Magazine, Vol. 39, Issue 5, pp. 90 - 98, May 2001.

[Cam99] Andrew T. Campbell, Herman G. De Meer, Michael E. Kounavis, Kazuho Mik, John B. Vicente, and Daniel ViUela, "A Survey of Programmable Networks", Proceedings of ACM SIGGCOMM, Vol. 29, Issue 2, April 1999. 
[Cho02] Chun Tung Chou, "Traffic Engineering for MPLS based Virtual Private Networks", Proceedings of IEEE INFOCOM, pp. 110-115, June 2002.

[Chu08] Jian Chu and Chin-Tau Lea, "New Architecture and Algorithms for Fast Construction of Hose-Model VPNs", IEEE/ACM Transactions on Networking, Vol. 16, No. 3, pp. 670-680, June 2008.

[CISCO] CISCO Telepresence Service.

http://www.cisco.com/en/US/netsol/ns669/networking_solutions_solution_segment_hom e.html

[Duf95] N. G. Duffield, Pawan Goyal, Albert Greenberg, Partho Mishra, K. K. Ramakrishnan, and Jacobus E. van der Merwe, "Resource Management with Hoses: Point-to-Cloud Services for Virtual Private Networks ", IEEE/ACM Transactions on Networking, Vol. 10, No. 5, pp. 679-692, Oct. 2002.

[Dut00] Rudra Dutta and George N. Rouskas, "A Survey of Virtual Topology Design Algorithms for Wavelength Routed Optical Networks," Tech. Rep. TR-99-06, Department of Computer Science, North Carolina State University, Raghleigh, NC, 1999.

[Fle99] Lisa K. Fleischer, “Approximating Fractional Multicommodity Flow Independent of the Number of Commodities", Proceedings of IEEE Annual Symposium of Foundations of Computer Science, pp. 24-31, 1999.

[Fon02] N.L.S. Da Fonseca, A.P. Castro, and A.T. Rios Jr., , "A Procedure for Resource Allocation in Switchlet Networks", Proceedings of IEEE GLOBECOM, Vol. 2, pp. 1885 -1888 , Nov. 2002. 
[Gar98] N. Garg and J. Konemann, "Faster and Simpler Algorithms for Multicommodity Flow and other Fractional Packing Problems", Annual Symposium on Foundations of Computer Science, IEEE, pp. 300-309, Nov. 1998.

[Gha06] N. Ghani, D. Benhaddou, W. Alanqar, V.M. Muthalay, and M. Hari, "Performance of Dynamic Shared Layer 1 VPN Services in Next-Generation SONET/SDH Networks", Proceedings of IEEE ICC, Vol. 6, pp. 2737 - 2742, June 2006.

[Gue97] R. Guerin and A. Orda, "QoS Routing in Networks with Inaccurate Information: theory and algorithms", IEEE/ACM Transactions on Networking, Vol. 7, Issue 3, pp. 350 - 364, June 1999.

[Gir00] M.K. Girish, B. Zhou, and J.Q. Hu, "Formulation of the Traffic Engineering problems in MPLS based IP networks," Proceedings of the $5^{\text {th }}$ International Symposium on Computers and Communications(ISCC 2000), pp. 214-219, 2000.

[Gun98] Manuel Günter, "Virtual Private Networks over the Internet", Paper on CATI Project by Swiss National Foundation.

[Gar00] Rahul Garg and Huzur Saran, "Fair Bandwidth Sharing in Virtual Networks: A capacity Resizing Approach", Proceedings of IEEE INFOCOM, Vol. 1, pp. 255 - 264, March 2000.

[Hyo03] Seung Hyong Rhee and Takis Konstantopoulos, "Dynamic Capacity Resizing for Fair Bandwidth Sharing in VPNs", IEICE Transaction Communication, Vol. E86-B, No.5, May 2003. 
[Hao00] Fang Hao and Ellen W. Zegura, “On Scalable Performance Routing: Evaluation of Topology Aggregation", Proceedings of IEEE INFOCOM, Vol. 1, pp. 147-156, March 2000.

[Ho07]Kwok Shing Ho and Kwok Wai Cheung, "Generalized Survivable Networks", IEEE/ACM Transactions on Networking, Vol. 15, No. 4, pp. 750 - 760, Aug. 2007.

[Isa00] Rebecca Isaacs and Ian Leslie, "Support for Resource-Assured and Dynamic Virtual Private Networks", IEEE Journal on Selected Areas in Communications, Vol. 19, No. 3, pp. 460-472, March 2001.

[Jut05] Alpár Juttner and Istvan Szabo "On Bandwidth Efficiency of Hose Resource Management in Virtual Private Networks", Proceedings of IEEE INFOCOM, Vol. 1 pp. 386-395, 2003.

[Kha00]Ibrahim Khalil and Torsten Braun, "Edge Provisioning and Fairness in VPNDiffServ Networks", Proceedings of IEEE ICC, pp. 424 - 431, Oct. 2000.

[Kum02] Amit Kumar, Rajeev Rastogi, and Avi Siberschatz, "Algorithms for Provisioning Virtual Private Networks in Hose Model", IEEE/ACM Transactions on Networking, Vol. 10, No. 4, pp. 565-578, Aug. 2002.

[Kou81] L. Kou, G. Markowsky, and L. Berman, "A Fast Algorithm for Steiner Trees", Acta Informatica, Springer-Verlag, Vol. 15, pp.141-145, 1981.

[Kis92] W. Kishimoto and M. Takeuchi, "On M-Route Flow in Networks", ICCS/ISITA, 1992. 
[Kod00] Murali S. Kodialam, T.V. Laxman and Sudipta Sengupta, "Online Multicast Routing with Bandwidth Gurantees: A New Approach Using Multicast Network Flow”, IEEE/ACM Transactions on Networking, Vol. 11, Issue 4, pp. 676-686, Aug. 2003.

[Kni04] Paul Knight and Chris Lewis, "Layer 2 and 3 Virtual Private Networks: Taxonomy Technology and Standardization Efforts", IEEE Communication Magazine, Vol. 42, Issue 6, pp. 124 - 131, June 2004.

[Kni06] Paul Knight and Hamid Ould-Brahim, "Network Based IP-VPN Using Virtual Routers", draft-ietf-13vpn-vpn-vr-03.txt, Internet Draft, IETF, March, 2006.

[Kor00] Turgay korkmaz and Marwan Krunz, "Source-Oriented Topology Aggregation with Multiple QoS parameters in Hierarchical Networks", ACM Transactions on Modeling and Computer Simulations, Vol. 10, No. 4, pp. 295-325, Oct. 2000.

[Lui04] King-Shan Lui, Klara Nahrstedt, and Shigang Chen, "Routing with Topology Aggregation in Delay-Bandwidth Sensitive Networks", IEEE/ACM Transactions on Networking, Vol. 12, No. 1, pp. 17-29, Feb. 2004.

[Law00] Averill M. Law and W. David Kelton, "Simulation Modelling and Analysis", McGraw Hill, Third Edition, 2000.

[Lee95] W. Lee, "Topology Aggregation for Hierarchical Routing in ATM networks", Proceedings of ACM SIGGCOMM Computer Communication, Vol. 25, pp. 82-92, April 1995.

[Lee01] W. Lee, "Spanning Tree Method for Link State Aggregation in Large Communication Networks", Proceedings of IEEE INFOCOM, pp. 82-90, Feb. 2001. 
[Lee99] W. Lee, "Minimum Equivalent Subspanner Algorithms for Topology Aggregation in ATM Networks", Proceedings of the 2nd International Conference on ATM (ICATM'99). IEEE Press, Piscataway, NJ, pp. 351-359, 1999.

[LINDO] LINDO Optimization Tool, (www.lindo.com).

[Met03] Chris Metz, "The Latest in Virtual Private Networks: Part 1", IEEE Internet Computing, Vol. 7, Issue 1, pp. 87-91, Jan-Feb 2003.

[Meh07] Deepankar Mehdi and Kartikeyan Ramaswamy, "Network Routing: Algorithms, Protocols and Architectures”, Morgan Kaufmann, 2007.

[Mit99] Debasis Mitra and Ilze Ziedins, "Hierarchical Virtual Partitioning: Algorithms for Virtual Private Networking", Proceedings of IEEE GLOBECOM, Vol. 3, pp. 1784 1791, Nov. 1997.

[Mer97] J. E. van der Merwe and I. M. Leslie, "Switchlets and Dynamic Virtual ATM Networks," Integrated Network Management, pp. 355-368, May 1997.

[Mer98] Jacobus E. van der Merwe, "Service Specific Control Architectures for ATM", IEEE Journal of Selected Area of Computing, Vol. 16, No. 3, pp. 424-436, April 1998.

[OPNET] Discrete Event Simulator, www.opnet.com.

[Pax95] V. Paxon and S. Floyd, "Wide Area Traffic: The Failure of Poisson Modeling", IEEE/ACM Transactions on Networking, Vol. 3, pp.226-244, June 1995. 
[Poh04] Tze-Ven Poh and Jing Jiang, "Multicommodity Flow Optimization in Support of Packet Switched Network Traffic Engineering", IEEE Workshop on IP Operations and Management, pp. 23-28, Oct. 2004.

[PNN196] "Private network-network interface specification version 1.0 (PNNI)", Technical report, The ATM forum technical committee, March 1996, af-pnni-0055.000.

[RFC1633] R.Braden and D.Clark, "Integrated Services in the Internet Architecture: an Overview", RFC1633, IETF, June 1994.

[RFC2328] J. Moy, “OSPF Version 2”, RFC 2328, IETF, April 1998.

[RFC3031] E. Rosen, A. Viswanathan et al, "Multiprotocol Label Switching Architecture ", RFC 3031, IETF, Jan. 2001.

[RFC3036] L. Andersson, P. Doolan et al, "Label Distribution Protocol Specification", RFC 3036, IETF, Jan. 2001.

[RFC3270] L. Wu, B. Davie et al, "Multi-Protocol Label Switching (MPLS) Support of Differentiated Services", RFC 3270, IETF, May 2002.

[RFC2404] S. Kent and R. Atkinson, "IP Authentication Header", RFC2404, IETF, Nov. 1998.

[RFC2406] S. Kent and R. Atkinson, "IP Encapsulation Security Payload", RFC2406, IETF, Nov. 1998.

[RFC2475] S. Blake, D. Black et al, "An Architecture for Differentiated Services", RFC 2475, IETF, Dec. 1998. 
[RFC2858] T. Bates, Y. Rekhter et al, "Multiprotocol Extensions for BGP-4", RFC 2858, IETF, June 2000.

[RFC3031] E. Rosen, A.Viswanathan et al, "Multiprotocol Label Switching Architecture", IETF Aug. 1999.

[RFC4364] E. Rosen and Y. Rekter, "BGP/MPLS Virtual Private Networks", RFC 4364, IETF, Feb. 2006.

[RFC3809] A. Nagarajan, "Generic Requirements for Provider Provisioned Virtual Private Networks (PPVPN)", RFC 3809, IETF, June, 2004.

[RFC3471] L. Berger, "Generalized Multi-Protocol Label Switching (GMPLS) Signaling Functional Description", RFC 3471, IETF, January, 2003.

[RFC3472] P. Ashwood-Smith and L. Berger, "Generalized Multi-Protocol Label Switching (GMPLS) Signaling Constraint-based Routed Label Distribution Protocol (CR-LDP) Extensions", RFC 3472, IETF, January, 2003.

[RFC3473] L. Berger, “Generalized Multi-Protocol Label Switching (GMPLS) Signaling Resource Reservation Protocol-Traffic Engineering (RSVP-TE) Extensions" RFC 3473, IETF, Jan. 2003.

[RFC4203] K.Kompella and Y.Rekhter, “OSPF Extensions in Support of Generalized Multi-Protocol Label Switching (GMPLS)” RFC 3473, IETF, Oct. 2005.

[RFC3945]E. Mannie, "Generalized Multi-Protocol Label Switching (GMPLS) Architecture", RFC 3945, IETF, Oct. 2004. 
[RFC4577] E. Rosen and P. Psenak, "OSPF as the Provider/Customer Edge Protocol for BGP/MPLS IP Virtual Private Networks (VPNs)", RFC 4577, IETF, Jan. 2006.

[RFC4847] T. Takeda, "Framework and Requirements for Layer 1 Virtual Private Networks", RFC 4847, IETF, April 2007.

[Rav04] Ravi Ravindran, Peter Ashwood-Smith, Hong Zhang, and Guo-Qiang Wang, "Multiple Abstraction Schemes for Generalized Virtual Private Networks", Proceedings of IEEE CCECE, Vol. 1, pp. 519 - 522, May 2004, Niagara.

[Rav05] Ravi Ravindran, Changcheng Huang, and K.Thulasiraman, "Topology Aggregation as a VPN Service", Proceedings of IEEE ICC, Vol. 1, pp. 105 - 109, May 2005, Seoul.

[Rav06] Ravi Ravindran, Changcheng Huang, and K.Thulasiraman, "A Dynamic Managed VPN Service: Architecture and Algorithms", Proceedings of IEEE ICC, Vol. 2, pp. 664 - 669, May 2006, Istanbul.

[Rav07] Ravi Ravindran, Changcheng Huang, and K.Thulasiraman, "A Dynamic Managed VPN Service: Capacity Sharing Based On Maximum Concurrent Flow Theory", Proceedings of IEEE ICC, Vol. 2, pp. 211 - 216, June 2007, Glasgow.

[Ram96] S. Ramanathan, "Multicast Tree Generation in Network with Asymmetric Link", IEEE/ACM Transaction on Networking, Vol. 4, Issue 4, pp. 558 - 568, Aug. 1996. 
[Sez03] W. Sezto, Y. Iraqi, and R. Boutaba, "A Multicommodity Based Approach to Virtual Network Resource Allocation Problem", Proceedings of IEEE GLOBECOM, Vol. 6, pp. $3004-3008$, Dec. 2003.

[Ste92] Clifford Stein, "Approximation Algorithms for Multi-Commodity Flow and Shop Scheduling Problem", Ph.D. Thesis, MIT, 1992.

[Sha90] Farhad Shahrokhi and D.W. Matula, "The Maximum Concurrent Flow Problem", Journal of the Association for Computing Machinery, Vol. 37, No. 2, pp. 318334, April 1990.

[Sar01] Venkatesh Sarangan and Raj Acharya, "A Study using Network Flows in Hierarchical QoS Routing", Proceedings of International Conference on Networks, IEEE, pp. $422-427$, Oct. 2001.

[Sar06] Venkatesh Sarangan and Jyh-Cheng Chen, "Comparative study of Protocols for Dynamic Service Negotiation in the Next-Generation Network", IEEE Communication Magazine, Vol. 44, Issue 3, pp. 151 - 156, March 2006.

[Tan05]Yong Tang and Shigang Chen, "QoS Information Approximation for Aggregated Networks", Proceedings of IEEE ICC, Vol. 4, pp. 2107 - 2111, June 2004.

[Tak04] Tomonori Takeda, Ichiro Inoue, D. Papadimitriou, and H. Ould-Brahim, "Layer 1 Virtual Private Networks: Service Concepts, Architecture Requirements, and Related Advances in Standardization", IEEE Communication Magazine, Vol. 43, Issue 7, pp. 60 -67 , July 2005.

[Wax88] B.M. Waxman, "Routing of multipoint Connections", Journal on Selected Areas in Communications, Vol. 6, pp. 1617-1622, Dec. 1988. 
[Wei04] Wei, "Implementing Fair bandwidth Resource Allocation Schemes in Hose modeled VPN", IEE Proceedings of Communication, Vol. 151, No. 6, Dec. 2004.

[Wan99] Y. Wang and Z. Wang, "Explicit routing algorithms for Internet trafec engineering," Proceedings of 8th Internationl Conferernce on Computer Communications and Networks (ICCN), pp. 582-588, 1999.

[Wan96] Z. Wang and J. Crowcroft, "Quality-of-Service Routing for Supporting Multimedia Applications," IEEE Journal on Selected Areas in Communications, Vol. 14, No.7, pp.1228-1234, Sept. 1996.

[Yua01] Ruixi Yuan and W.Timothy Strayer, "Virtual Private Networks, Technologies and Solutions", Addison-Wesley, 2001.

[Zen03] Jingdi Zeng and Nirwan Ansari, "Towards IP Virtual Private Network Quality of Service: A Service Provider Perspective", IEEE Communication Magazine, Vol. 41, Issue 4, pp. 113 - 119, April 2003. 